\title{
WestVirginiaUniversity
}

THE RESEARCH REPOSITORY @ WVU

Graduate Theses, Dissertations, and Problem Reports

2001

\section{Large -eddy simulation of ship wakes}

Shaoping Shi

West Virginia University

Follow this and additional works at: https://researchrepository.wvu.edu/etd

\section{Recommended Citation}

Shi, Shaoping, "Large -eddy simulation of ship wakes" (2001). Graduate Theses, Dissertations, and Problem Reports. 2401.

https://researchrepository.wvu.edu/etd/2401

This Dissertation is protected by copyright and/or related rights. It has been brought to you by the The Research Repository @ WVU with permission from the rights-holder(s). You are free to use this Dissertation in any way that is permitted by the copyright and related rights legislation that applies to your use. For other uses you must obtain permission from the rights-holder(s) directly, unless additional rights are indicated by a Creative Commons license in the record and/ or on the work itself. This Dissertation has been accepted for inclusion in WVU Graduate Theses, Dissertations, and Problem Reports collection by an authorized administrator of The Research Repository @ WVU.

For more information, please contact researchrepository@mail.wvu.edu. 


\title{
LARGE-EDDY SIMULATION OF SHIP WAKES
}

\author{
Shaoping Shi \\ Dissertation submitted to the \\ College of Engineering and Mineral Resources \\ at West Virginia University \\ in partial fulfillment of the requirements \\ for the degree of \\ Doctor of Philosophy \\ in \\ Mechanical Engineering \\ Ismail Celik, Ph.D., Chair \\ John Kuhlman, Ph.D. \\ Andrei Smirnov, Ph.D. \\ Steve Lewellen, Ph.D. \\ Stephen Jordan, Ph.D. \\ Ian Christie, Ph.D.
}

Mechanical and Aerospace Engineering Department

Morgantown, West Virginia

2001

Keywords: Large-eddy simulation, Subgrid-scale models, Ship wake flows

Copyright 2001 by Shaoping Shi 


\section{ABSTRACT \\ LARGE-EDDY SIMULATION OF SHIP WAKES}

\section{Shaoping Shi}

The objective of the present study is to improve our understanding of turbulent wake flows. Large-Eddy Simulation (LES) technique is applied for this purpose. A readily available code was used with necessary modifications. Three dimensional incompressible Navier-Stokes equations are solved in non-orthogonal curvilinear coordinates. Finitevolume approach is implemented on a non-staggered grid. The core of the numerical scheme is a fractional step method. The overall accuracy of the method is second order in both space and time.

The LES approach has been validated for four cases: channel flow , flow past a square body, a shear layer flow, and open channel flow. Different subgrid-scale models and numerical schemes have been tested for these benchmarks. Comparisons between the simulations and experiments show the capability of this LES method.

An efficient and accurate Random Flow Generation (RFG) approach has been improved to provide turbulent initial and inflow conditions for developing wake flow calculations. The RFG method can handle anisotropy and inhomogeneity, and it satisfies the instantaneous continuity equations. This approach has been verified by reproducing a turbulent channel flow based on a Direct Numerical Simulation (DNS). Perfect agreements have been obtained.

A typical one equation sub-grid scale model has been selected and modified to include the backscatter of energy by applying the RFG algorithm. Meanwhile, an attempt was made to implement the one equation sub-grid scale model dynamically. Comparisons have been made between the experiments and the simulation results using different one equation sub-grid scale models. The RFG approach along with the LES technique has been applied to the wake behind a flat plate. Effects of grid resolution and SGS models on the turbulent flow field have been investigated. Good results are obtained as compared to experiments.

Wake flows behind a Naval ship model (5415) have been studied in details by applying the combined LES-RFG method. The calculation domain starts from a plane behind the ship model. Because of the lack of experimental data, Reynolds Averaged Navier-Stokes (RANS) calculations are used to provide the RFG procedure with the information on the inflow boundary. The ship wake flow with an impose surface wave has been studied. The result shows that the wave surface has a significant influence on the turbulent kinetic energy distribution. Finally, part of the ship hull is included in the simulation of the ship wake with a static wave surface $(\mathrm{Fr}=0.28)$ to investigate the difference between RANS and LES results in the near wake of a ship model. The overall quality of the LES calculations is found to be very good. In particular the large coherent structures with significant vorticity concentration and gradients could be captured in the wake of a ship model which is not possible to accomplished by RANS. These structures have significant importance in the transplant of entrained air bubbles. 


\section{Acknowledgements}

Completion of this dissertation would have been impossible for me without the guidance and help from my advisors, colleagues, friends and family.

I am indeed indebted to a number of individuals for their support and advice during this period. Foremost, I would like to thank Dr. Ismail Celik, my advisor, for his support and encouragement. His high attainments in mathematics, keen insight in physics, enthusiasm and optimistic view of research and life have constantly inspired me.

I offer my profound gratitude to Dr. Andrei Smirnov. He deserves high credit for providing numerous hours of free consultation and kindly guidance through all my study in WVU. Dr. John Kuhlman has my grateful acknowledgments for his patience with me and for his wonderful guidance in the world of Fluids Mechanics. Special thanks are also due to Dr. Steve Lewellen, Dr. Stephen Jordan and Dr. Ian Christie for providing important perspectives to my research and insightful suggestions in the face of my simulations.

I would express my deep appreciation to Dr. Robert Street of Stanford University for providing the LES code. I am grateful to Dr. Ron Calhoun and Mr. Li Ding for their kindly help on using this code. I thank Dr. Fred Stern, Dr. Eric Paterson, Dr. Robert Wilson, and Dr. Joe Longo of Iowa University, Dr. Mark Hyman of Navy for providing their valuable data.

I thank my office mate. Dr. Ibrahim Yavuz and Dr. Aytekin Gel, for their friendship and their help on setting the computers and using softwares. Also, my thanks are given to Andrew Burt, Allen Badeau, Sherif Kandil, Zeynep Cehreli, Wesley Wilson, and all of my lab mates and friends, who made my stay a great experience.

I am specially grateful to my parents and family in my homeland China. Their love and encouragement to me have always been great sources of strength.

Most importantly, I thank my wife, Rong. She made many sacrifices in helping me carry on this research in the last several years. Her constant love and support have made my life happy and fulfilling.

This work has been performed under a DOD EPSCoR project sponsored by the Office of Naval Research (ONR), Grant No.N00014-98-1-0611. The program monitor was Dr. Edwin P. Rood/Patrick L. Purtell. Their financial support is gratefully acknowledged. 


\section{Nomenclature}

\section{Roman Symbols}

$\begin{array}{ll}a_{i j} & \text { a transfer coefficient tensor } \\ A & \text { coefficients } \\ B_{i} & \text { discrete operator for the pressure gradient } \\ C & \text { coefficients } \\ D & \text { side length of the square cylinder } \\ F_{i j} & \text { flux in the momentum equation } \\ F r & \text { Froude number } \\ G^{m n} & \text { mesh skewness tensor } \\ g & \text { gravitational acceleration } \\ J & \text { Jacobian } \\ k & \text { turbulent kinetic energy, wave number } \\ K & \text { SGS turbulent kinetic energy on the test filter } \\ l & \text { turbulence length scale } \\ L & \text { ship length } \\ L_{i j} & \text { Leonard term } \\ L_{i j}^{T} & \text { modified Leonard term of the test scale } \\ N & \text { number of grid points in one dimension } \\ P & \text { pressure } \\ p & \text { reduced dynamic pressure } \\ q & \text { a generic variable for filterring } \\ r_{i j} & \text { anisotropic velocity correlation tensor } \\ R_{j} & \text { flux in the scalar equation } \\ R_{e} & \text { Reynolds number } \\ R_{y} & \text { turbulence Reynolds number } \\ S_{i} & \text { source term of the momentum equation and scalar equation } \\ S_{T i} & \text { modified source term of the scalar equation } \\ \bar{S}_{i j} & \text { resolved strain rate tensor } \\ T_{i j} & \text { sub-test-scale stress } \\ t & \text { time } \\ U_{m} & \text { volume flux } \\ u_{\tau} & \text { friction velocity } \\ u, v, w & \text { Cartesian velocities in } x, y \text { and } z \text { direction } \\ & \end{array}$




$\begin{array}{ll}v & \text { subgrid-scale fluctuations } \\ X_{j} & \text { sub-test-scale scale flux } \\ x_{i},(x, y, z) & \text { Cartesian coordinates } \\ y & \text { distance to the wall }\end{array}$

\section{Greek Symbols}

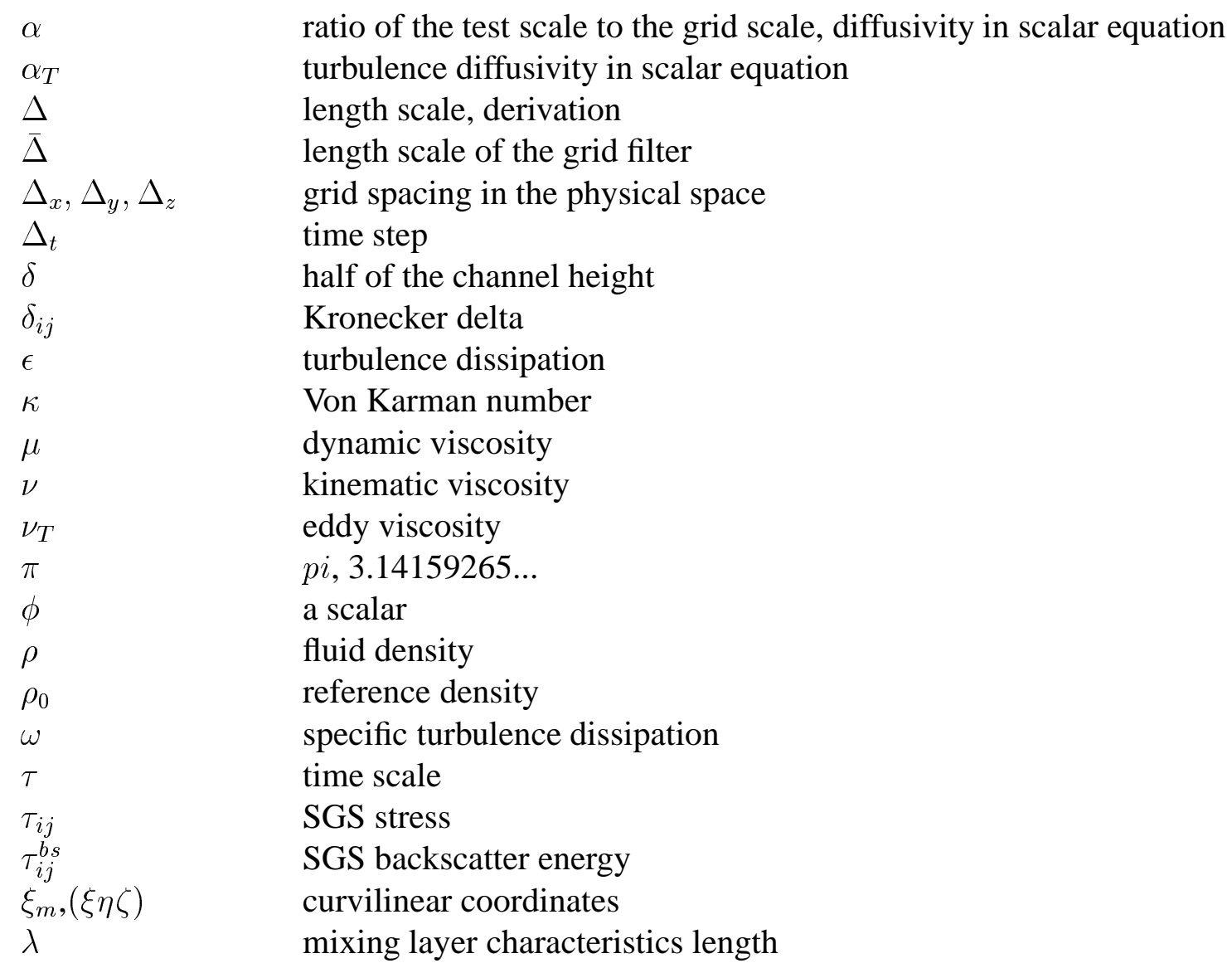

\section{Other Symbols}

$\begin{array}{ll}\partial & \text { partial derivative operator } \\ \delta & \text { discrete derivative operator } \\ \cong & \text { approximately equal } \\ \sim & \text { order of magnitude } \\ \sigma & \text { summation } \\ \Pi & \text { product }\end{array}$

\section{Superscripts}




$\begin{array}{ll}+ & \text { nondimensional quantity } \\ b s & \text { backscatter } \\ n & \text { index of the time step } \\ p & \text { index of the pressure iteration } \\ ()^{\prime} & \text { a SGS or spatial perturbation quantity }\end{array}$

\section{Subscripts}
$i, j, k$
indices for the Cartesian coordinates or vector quantities
$m$
indices for the curvilinear coordinates or vector quantities
$w$
wall

\section{Abbreviations}

DMM

RFG

DNS

DSM

LES

SGS

CD

QUICK

ISM

ESM

RANS dynamic mixed model

Random flow generation

direct numerical simulation

dynamic subgrid-scale eddy viscosity model

large eddy simulation

subgrid scale

central differencing

Quadratic Upstream Interpolation for Convective Kinematics

Implicit Superimposition Method

Explicit Superimposition Method

Reynolds Averaged Navier Stokes 


\section{Contents}

$\begin{array}{ll}\text { Abstract } & \text { ii }\end{array}$

Acknowledgements

Nomenclature iv

1 Introduction 1

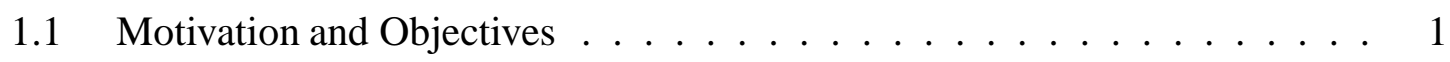

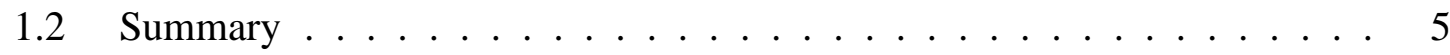

2 Mathematical Formulation 6

2.1 Governing Equations . . . . . . . . . . . . . . . . 6

2.2 Boussinesq Approximation . . . . . . . . . . . . . . . 7

2.3 Conservation Law Form . . . . . . . . . . . . . . . . . 8

2.4 Equations for the Resolved Motion . . . . . . . . . . . . . . . . . 9

2.5 Coordinate Transformation ................... 13

3 Numerical Method and Validation 16

3.1 Summary of Numerical Method . . . . . . . . . . . . . . . . . . 17

3.2 Subgrid Scale Model . . . . . . . . . . . . . . . . . . . . 18

3.3 LES of Channel Flow . . . . . . . . . . . . . . . . . . . . . 19

3.4 LES of Flow Past Square Cylinder . . . . . . . . . . . . . . . . 21

3.5 LES of Plane Mixing Layer . . . . . . . . . . . . . . . . . . . . . . . . . . . . . . . . . . . . .

3.6 LES of Open Channel Flow . . . . . . . . . . . . . . . . . . . . . . . . . . . . . . . . 26

3.7 Summary . . . . . . . . . . . . . . . . . . 27

4 Random Flow Generation $\quad 44$

4.1 Introduction . . . . . . . . . . . . . . . . 44

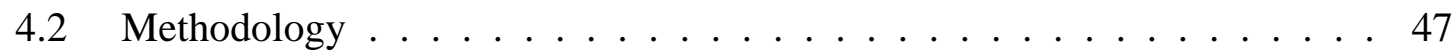

4.3 Determination of the Length Scale and Time Scale . . . . . . . . . . . 50

4.4 Validation of RFG . . . . . . . . . . . . . . . . . . . . . . . . . . . . . 51

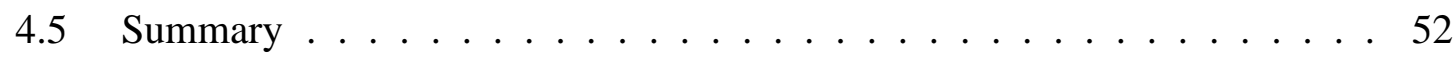


5 One Equation Subgrid-scale Model 58

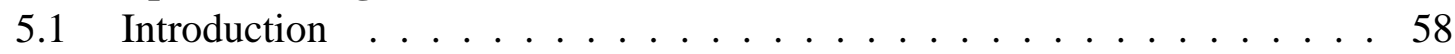

5.2 Subgrid-scale Kinetic Energy Equation _. . . . . . . . . . . . . 62

5.3 Solution to the SGS Turbulent Kinetic Energy Equation . . . . . . . . 66

5.4 Near Wall Modification . . . . . . . . . . . . . . . . . . . . . . 67

5.5 Calculation of the Backscatter Energy _. . . . . . . . . . . 68

5.5.1 Superimposition of the velocity fluctuations on the resolved flow field —Explicit Superimposition Method (ESM) . . . . . . . 69

5.5.2 Superimposition of backscatter stresses in both $k$-equation and momentum equation —-Implicit Superimposition Method (ISM) 70

5.6 Application to the Channel Flow . . . . . . . . . . . . 71

5.6.1 Results of one equation model . . . . . . . . . . . 72

5.6.2 Backscatter results and discussion: approach $1 \ldots \ldots$. . . . . 74

5.6.3 Backscatter results and discussion: approach $2 \ldots \ldots \ldots 75$

$5.7 \quad$ Summary . . . . . . . . . . . . . . . . . . . . . . 77

6 Applications to Flat Plate Wake 92

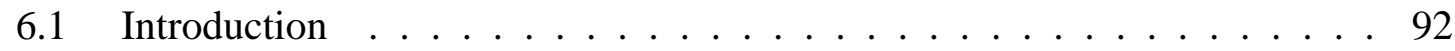

6.2 Details of Simulations . . . . . . . . . . . . . . . . . 93

6.3 Inflow Boundary . . . . . . . . . . . . . . . . . . 93

6.4 Instantaneous Flow Field . . . . . . . . . . . . . . . . . . . . 94

6.5 Mean Flow Quantities . . . . . . . . . . . . . . . . . . . 95

6.6 Reynolds Stresses . . . . . . . . . . . . . . . . . . . . . . 96

6.7 Sensitivity to Grid Resolution . . . . . . . . . . . . . . . . . 97

6.8 Effects of SGS models . . . . . . . . . . . . . . . . . . . 97

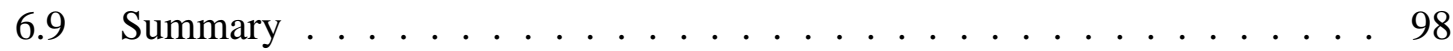

7 Application to Ship Wake 119

7.1 Introduction . . . . . . . . . . . . . . . . . . . . . . 119

7.1.1 Experiments on turbulent ship wake flows . . . . . . . 120

7.1.2 Computations of turbulent ship wake flows . . . . . . . . 120

7.2 Ship Model DTMB $5415 \ldots \ldots$. . . . . . . . . . . . . . . . 124

7.3 Designing of Cases Studied . . . . . . . . . . . . . . . . 124

7.3.1 Case 1: Ship wake with flat free-surface . . . . . . . . . . . 124

7.3.2 Case 2: Ship wake with sinusoidal free surface . . . . . . . 126

7.3.3 Case 3: Simulations including part of the ship hull with wave free

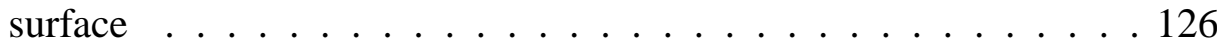

7.4 Results and Discussion . . . . . . . . . . . . . . . . . . 128

7.4.1 Ship wake with flat surface . . . . . . . . . . . . 128

7.4.2 Ship wake with sinusoidal free surface . . . . . . . . . . 131

7.4.3 Ship wake flows including part of the ship hull with free surface waves . . . . . . . . . . . . . . . . 131 


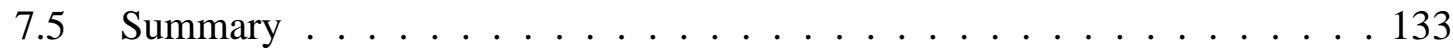

8 Conclusions and Recommendations 163

8.1 Conclusions . . . . . . . . . . . . . . . . . 163

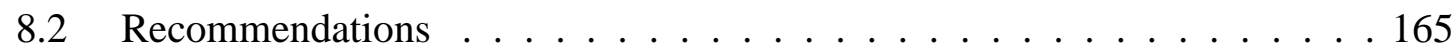

$\begin{array}{lll}\text { A Parallel Computation } & 167\end{array}$

A.1 Domain Decomposition Strategy . . . . . . . . . . . . . . . . 167

A.2 Implementation on the Beowulf cluster . . . . . . . . . . . . . . . 169

A.3 Test Simulation . . . . . . . . . . . . . . . . . . 171

A.4 Scalability Analysis . . . . . . . . . . . . . . . . . . . . . . . . . . . . . . . . . . . . . .

A.5 Summary . . . . . . . . . . . . . . . . . . 172

B Introduction of the LES Code $\quad 178$

B.1 Flow Chart . . . . . . . . . . . . . . . . . . . . . . 178

B.2 Description of the Subroutines . . . . . . . . . . . . 181

B.3 Using the Code . . . . . . . . . . . . . . . . . . 189

C Calculation of the Energy Spectra 194 


\section{List of Tables}

5.1 Parameters for large eddy simulation of channel flow using One-equation

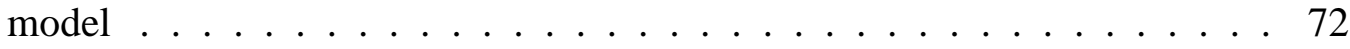

A.1 Parabolic domain decomposition algorithm . . . . . . . . . . . 168

A.2 Elliptic domain decomposition algorithm . . . . . . . . . . . . 170 


\section{List of Figures}

3.1 Resolved and modeled energy spectra $\ldots \ldots \ldots \ldots . \ldots 28$

3.2 Overactive model and underactive model $\ldots \ldots \ldots \ldots . \ldots 28$

3.3 The geometry of the channel flow . . . . . . . . . . . . . . . . 29

3.4 The geometry of the flow past a square cylinder . . . . . . . . . . . 29

3.5 Mean flow logarithmic velocity profile in the channel flow . . . . . . . 30

3.6 The turbulence intensity for the channel flow . . . . . . . . . . 30

3.7 Contours on the center $x-y$ plane . . . . . . . . . . . . . . 31

3.8 Mean velocity on the center plane of the cylinder . . . . . . . . . 32

3.9 Turbulence intensity of the flow around the square cylinder . . . . . . . . 33

3.10 Contours on center $x-y$ plane . . . . . . . . . . . . . . . . 34

3.11 Energy spectrum of the square cylinder $\ldots \ldots \ldots \ldots \ldots$

3.12 The sketch of plane mixing layer flow . . . . . . . . . . . . 36

3.13 Mean and rms of streamwise velocity in mixing layer, streamwise . . . . 37

3.14 Mean and rms of streamwise velocity in mixing layer, vertical $\ldots \ldots .37$

3.15 Velocity vectors at $x / \lambda=1.25 \ldots \ldots \ldots \ldots \ldots$

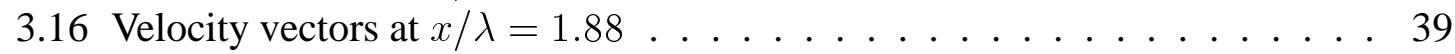

3.17 Velocity vectors at $x / \lambda=2.50 \ldots \ldots \ldots \ldots \ldots \ldots$

3.18 Vertical velocity contours . . . . . . . . . . . . . . 41

3.19 Bubble trajectories in the mixing layer . . . . . . . . . . . . 41

3.20 The mean streamwise velocity in the open channel flow . . . . . . 42

3.21 The contours for open channel flow, $u \ldots \ldots \ldots \ldots$. . . . . . . 42

3.22 The contours for open channel flow, $w \ldots \ldots \ldots \ldots$. . . . . . 43

4.1 Contours of the u-velocity at $x-y$ plane $\ldots \ldots \ldots \ldots \ldots$

4.2 Contours of the u-velocity at $x-z$ plane parallel to the wall $\ldots \ldots \ldots 5$

4.3 The mean velocity profile, $\circ$ : DNS; —

4.4 The Reynolds stresses . . . . . . . . . . . . . . . . . . . . 56

4.5 The energy spectra of axial velocity fluctuations . . . . . . . . . 57

5.1 The mean velocity. $\longrightarrow$, case $I V ;-\ldots--$, case $I I I \ldots \ldots \ldots$

5.2 The mean subgrid scale energy $\ldots \ldots \ldots \ldots$. . . . . . . . . . 78

5.3 The mean turbulence viscosity . . . . . . . . . . . . . . . . . 79

5.4 The turbulence intensity $u_{r m s} .-\longrightarrow$, case $I I ;----$, case $I$; $\circ$, measurements (Hussain \& Reynolds, 1975). . . . . . . . . . . . . . . . 79 
5.5 The turbulence intensity $v_{r m s} \ldots \ldots \ldots \ldots \ldots$

5.6 The turbulence intensity $w_{r m s} \ldots \ldots \ldots \ldots$

5.7 The energy spectra $\ldots \ldots \ldots \ldots \ldots \ldots \ldots$

5.8 The resolved TKE and subgrid-scale TKE $\ldots \ldots \ldots \ldots$. . . . . 81

5.9 The mean streamwise velocity, ESM . . . . . . . . . . . . . . 82

5.10 The turbulence intensity $u_{r m s}$, ESM $\ldots \ldots \ldots \ldots 2$

5.11 The turbulence intensity $v_{r m s}$, ESM $\ldots \ldots \ldots \ldots \ldots$

5.12 The turbulence intensity $w_{r m s}$, ESM $\ldots \ldots \ldots \ldots$

5.13 The subgrid scale turbulent kinetic energy, ESM . . . . . . . . . . . 84

5.14 The subgrid scale turbulent kinetic energy, no backscatter . . . . . . . . . 84

5.15 The streamwise velocity contours, ESM . . . . . . . . . . . 85

5.16 The streamwise velocity contours, no backscatter $\ldots \ldots \ldots \ldots$. . . 85

5.17 The spanwise velocity contours, ESM _ . . . . . . . . . . . 86

5.18 The spanwise velocity contours, no backscatter . . . . . . . . . . 86

5.19 The spanwise velocity contours, ESM . . . . . . . . . . . . . 87

5.20 The spanwise velocity contours, no backscatter . . . . . . . . . . . 87

5.21 The mean streamwise velocity with backscatter,ISM . . . . . . . . . 88

5.22 The turbulence intensity $u_{r m s}$, ISM . . . . . . . . . . . . . . 88

5.23 The turbulence intensity $v_{r m s}$, ISM . . . . . . . . . . . . . . . . 89

5.24 The turbulence intensity $w_{r m s}$, ISM $\ldots \ldots \ldots$

5.25 The subgrid scale turbulent kinetic energy, ISM $\ldots \ldots \ldots$

5.26 The streamwise velocity contours, ISM . . . . . . . . . . . . . 90

5.27 The spanwise velocity contours, ISM . . . . . . . . . . . . . . 91

5.28 The spanwise velocity contours, ISM . . . . . . . . . . . . . 91

6.1 The schematic of the flat plate wake . . . . . . . . . . . . 100

6.2 The velocities at the inflow boundary . . . . . . . . . . . . . 101

6.3 Turbulence intensities at the inflow boundary, $u_{r m s} \ldots \ldots \ldots$. . . . . 102

6.4 Turbulence intensities at the inflow boundary, $v_{r m s} . \ldots \ldots \ldots$. . . . . 102

6.5 Turbulence intensities at the inflow boundary, $w_{r m s} . \ldots \ldots . \ldots 103$

6.6 Turbulence intensities at the inflow boundary, $u v_{r m s} . \quad \ldots \ldots$. . . . . 103

6.7 Typical streamwise vorticities $(\omega) \ldots \ldots \ldots$

6.8 Temporal history of the instantaneous velocity . . . . . . . . . . . 105

6.9 Energy spectrum at the same point as $\mathrm{x}=0$ in Fig.6.8 $\ldots \ldots \ldots \ldots$

6.10 Energy spectrum at the same point as $\mathrm{x}=0.16$ in Fig.6.8 $\ldots \ldots \ldots \ldots$

6.11 Energy spectrum at the same point as $\mathrm{x}=0.53$ in Fig.6.8 . . . . . . . 107

6.12 Comparison of the turbulence structures between LES and experiments . . 108

6.13 Centerline velocity in the wake of flat plate wake . . . . . . . . . . 109

6.14 U-component mean-velocity profile in the wake of the flat plate . . . . 109

6.15 Centerline velocity in the wake of flat plate wake (for testing ergodicity). . 110

6.16 Comparison between simulation and measured turbulence intensity, $\left(u_{r m s}\right) .110$

6.17 Comparison between simulation and measured turbulence intensity $\left(v_{r m s}\right)$. 111

6.18 Comparison between simulation and measured turbulence intensity $\left(w_{r m s}\right) .112$ 
6.19 Comparison between simulation and measured shear stresses . . . . . . . . 113

6.20 Turbulent kinetic energy profile along the center line in the wake of a flat

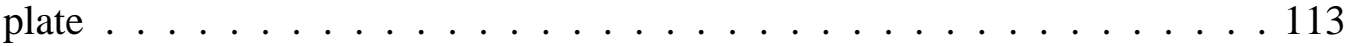

6.21 Turbulence intensity $\left(u_{r m s}\right)$ with finer grid . . . . . . . . . . . . . . 114

6.22 Turbulence intensity $\left(v_{r m s}\right)$ with finer grid . . . . . . . . . . . . . . . . . 114

6.23 Turbulence intensity $\left(w_{r m s}\right)$ with finer grid . . . . . . . . . . . . 115

6.24 Turbulence intensity $(\overline{u v})$ with finer grid . . . . . . . . . . . . . . . 115

6.25 Turbulent kinetic energy profile with one equation model . . . . . . . . 116

6.26 Turbulent kinetic energy profile along y direction . . . . . . . . . . . . . 116

6.27 Turbulence intensity $\left(u_{r m s}\right)$ with one equation model $\ldots$. . . . . . . . . 117

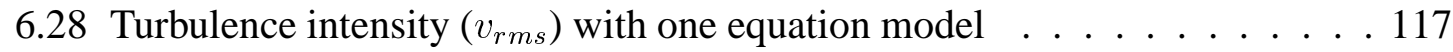

6.29 Turbulence intensity $\left(w_{r m s}\right)$ with one equation model . . . . . . . . . . . 118

6.30 Turbulence intensity $(u v)$ with one equation model . . . . . . . . . . . . 118

7.1 Hydrodynamic wake schematic . . . . . . . . . . . . . . . . 134

7.2 Geometry of DTMD $5415 \ldots \ldots \ldots \ldots$. . . . . . . . . . . . . . . . . . . . . . . . .

7.3 Ship wake simulations setup . . . . . . . . . . . . . . . . . . . . . 135

7.4 Ship wake simulations setup with surface waves . . . . . . . . . 136

7.5 The sketch of ship wake including part of the ship hull and wave surface . . 137

7.6 The mean streamwise velocity at the inlet plane for case $1 \ldots$. . . . . 137

7.7 The mean vertical velocity at the inlet plane for case $1 \ldots \ldots$. . . . . . 138

7.8 The mean spanwise velocity at the inlet plane for case $1 \ldots \ldots$. . . . 138

7.9 The mean velocity vectors at the inlet plane for case $1 \ldots$. . . . . . . 139

7.10 The mean turbulent kinetic energy at the inlet plane for case 1 . . . . . . . 139

7.11 Typical instantaneous streamwise velocity at the inlet plane for case 1 . . 140

7.12 Typical instantaneous vertical velocity at the inlet plane for case 1 . . . . . 140

7.13 Typical instantaneous spanwise velocity at the inlet plane for case 1 . . . 141

7.14 Typical instantaneous velocity vectors at the inlet plane for case 1 . . . . . 141

7.15 Typical instantaneous streamwise vorticity $\left(\omega_{x}\right)$ contours on different y-z plane in the ship wake . . . . . . . . . . . . . . . . . 142

7.16 Typical instantaneous vorticity contours $\left(\omega_{y}\right)$ on a x-z plane parallel to free surface at $y / L=-0.01$ in the ship wake . . . . . . . . . . . . . . . 142

7.17 Typical instantaneous streamwise velocity contours on a $\mathrm{X}-\mathrm{Z}$ plane parallel to free surface at $y / L=-0.01$ in the ship wake . . . . . . . . . . 143

7.18 The same as Fig.7.16 but double grid number in streamwise direction . . . 143

7.19 Comparison of resolved turbulence kinetic energy for different computing

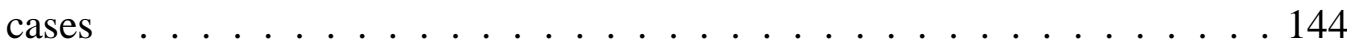

7.20 Typical instantaneous velocity vectors on $y-z$ plane at $x / L=0.2$ in the ship

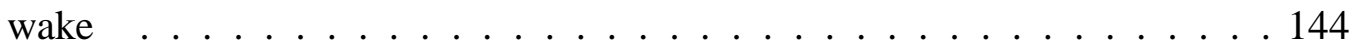

7.21 Typical instantaneous velocity vectors on $y-z$ plane at $\mathrm{x} / \mathrm{L}=0.6$ in the ship

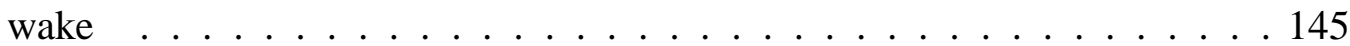

7.22 Typical instantaneous velocity vectors on $y-z$ plane at $x / L=1.0$ in the ship

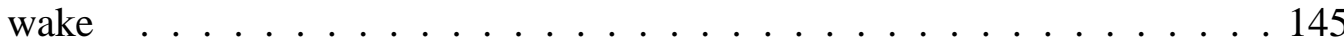


7.23 Typical instantaneous velocity vectors and the enlargement of area A on $\mathrm{y}$-z plane at $\mathrm{x} / \mathrm{L}=1.2$ in the ship wake . . . . . . . . . . 146

7.24 Typical instantaneous velocity vectors on $y-z$ plane at $x / L=1.4$ in the ship wake . . . . . . . . . . . . . . . . . . . 147

7.25 Typical instantaneous vorticity contours $\left(\omega_{x}\right)$ on y-z plane at $x / L=0.2$ in the ship wake . . . . . . . . . . . . . . . . . . . . 147

7.26 Typical instantaneous vorticity contours $\left(\omega_{x}\right)$ on $y-z$ plane at $x / L=0.6$ in the ship wake . . . . . . . . . . . . . . . . . 148

7.27 Typical instantaneous vorticity contours $\left(\omega_{x}\right)$ on $y-z$ plane at $x / L=1.2$ in the ship wake . . . . . . . . . . . . . . . . . . 148

7.28 Resolved turbulent kinetic energy at $\mathrm{Y} / \mathrm{L}=-0.02 \ldots \ldots \ldots$

7.29 The mean streamwise velocity at the inlet plane for case $3 \ldots \ldots$. . . . 150

7.30 The detail information of area $\mathbf{A}$ in Fig. $7.29 \ldots \ldots$

7.31 The mean vertical velocity at the inlet plane for case $2 \ldots \ldots \ldots 151$

7.32 The mean spanwise velocity at the inlet plane for case $3 \ldots \ldots . \ldots 151$

7.33 The mean velocity vectors at the inlet plane for case $3 \ldots \ldots . . . . .152$

7.34 The turbulent kinetic energy at the inlet plane for case $3 \ldots \ldots . \ldots 152$

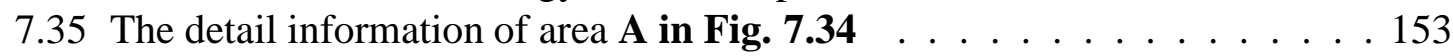

7.36 The instantaneous streamwise velocity at the inlet plane for case $3 \ldots 153$

7.37 The instantaneous vertical velocity at the inlet plane for case $3 \ldots \ldots 154$

7.38 The instantaneous spanwise velocity at the inlet plane for case $3 \ldots 154$

7.39 The instantaneous velocity vectors at the inlet plane for case $3 \ldots \ldots 155$

7.40 The instantaneous streamwise velocity at $x / L=0.935$ for case $3 \ldots 155$

7.41 The mean streamwise velocity at $x / L=0.935$ from measurements (Stern and Longo, 2001) . . . . . . . . . . . . . . . . . 156

7.42 The instantaneous vertical velocity at $x / L=0.935$ for case $3 \ldots \ldots 156$

7.43 The mean vertical velocity at $x / L=0.935$ from measurements (Stern and Longo, 2001) . . . . . . . . . . . . . . . . . . 157

7.44 The instantaneous spanwise velocity at $x / L=0.935$ for case $3 \ldots 158$

7.45 The mean spanwise velocity at $x / L=0.935$ from measurements (Stern and Longo, 2001) . . . . . . . . . . . . . . . . . . 158

7.46 The instantaneous velocity vectors at $x / L=0.935$ for case $3 \ldots 159$

7.47 The mean velocity vectors at $x / L=0.935$ from measurements (Stern and Longo, 2001) . . . . . . . . . . . . . . . . . . . 159

7.48 The instantaneous streamwise velocity at the free surface for case $3 \ldots 160$

7.49 The instantaneous vertical velocity at the free surface for case $3 \ldots 160$

7.50 The instantaneous spanwise velocity at the free surface for case $3 \ldots 161$

7.51 The instantaneous spanwise velocity at the free surface for case $3 \ldots 161$

7.52 The instantaneous streamwise velocity at the center vertical plane for case 3162

7.53 The instantaneous spanwise vorticity at the center vertical plane for case $3 \quad 162$

A.1 Domain decomposition strategy $\ldots \ldots \ldots \ldots$. . . . . . . . . 173

A.2 Wake decomposition . . . . . . . . . . . . . . . 174 
A.3 The demonstration of domain decomposition scheme . . . . . . . . . . 175

A.4 The streamwise velocity contours . . . . . . . . . . . . . . 176

A.5 A cluster of DEC-Alpha workstations at the CFD lab of WVU . . . . . . 177

B.1 The flow chart of the LES code - A . . . . . . . . . . . . . 179

B.2 The flow chart of the LES code - B . . . . . . . . . . . . . . 180

C.1 The sinusoidal signals . . . . . . . . . . . . . . . . . 198

C.2 The energy spectra of sinusoidal signals . . . . . . . . . . . . . . . . . . . . . . . . . . . . . . . . . .

C.3 The energy spectra of the test data . . . . . . . . . . . . . 199 


\section{Chapter 1}

\section{Introduction}

\subsection{Motivation and Objectives}

Turbulent wake flows are an important area of the Naval hydrodynamic research. They strongly effect the bubble dynamics, such as bubble trajectories and bubble concentrations, in the wake. They also constitute a significant part of the geo-system, including the global climate and our local environments. In 1978, a NASA scientific satellite, SEASAT equipped with a synthetic aperture radar (SAR) spent approximately five months in a nearEarth orbit. Analysis of scenes from SEASAT revealed a large number of ship wakes as well as other ocean features (Hoekstra, 1991). Many of these images revealed some fairly consistent wake features as well as indications to potential military and commercial implications resulting from either detection of ships and their wakes or to possible technological developments as to minimize, under a given set of environmental conditions, the wake scar left by a passing ship.

Most of the ship wake flows are turbulent. Several types of research directions were pursued to study wake flows, including field observations, as well as experimental and analytical methods. However, due to the big scale and complexity of ship wake flows 
especially those associated with the effects of the free surface, very few data are available so far, including those for a ship model instead of real ship. On the other hand, with the fast development of supercomputers, numerical simulation has been emerging as an increasingly effective tool of system optimization and design. When used as a prediction approach it can provide very useful information for ship designers, whereas as a postdiction approach it can give us some information on operating the ship so that it could lead to the reduction of energy consumption or required power.

However, numerical simulation of ship wake flows is still in its infancy, although some can be found, for example, in Dommermuth and Novikov (1993), Dommermuth et al. (1996), Hyman (1998; 1995), and Paterson et al.(1996). So far, most simulations have used Reynolds Averaged Navier-Stokes (RANS) methods. One of the well knowns is CFDSHIPIOWA (Paterson et al., 1998; Sotiropoulos and Patel, 1995).

LES is becoming a popular tool attributing to its obvious advantage and the fast increase in computer resources. From its beginning in the 60's (Lilly, 1967), LES had been successfully applied to weather prediction. It then came to the attention of engineering research. The works in the literature include but are not limited to: channel flows (Moin and Kim, 1982; Kim et al., 1987), flow past cylinders (Jordan and Ragab, 1998; Bosch and Rodi, 1998; Sohankar et al., 2000), backward facing step (Avancha and Pletcher, 2000), and jet (Akselvoll and Moin, 1996). The objective of LES is to compute the three-dimensional time-dependent details of the relatively large scales of motion (those responsible for the primary transport) while the small scales are modeled with an appropriate sub-grid scale (SGS) model. LES is intended to be used in the study of transient and turbulent flows at moderately high Reynolds numbers, in the development of turbulence models, and for predicting flows of technical interest in demanding complex situations where simpler modeling approaches (e.g. Reynolds stress transport) are inadequate. The most critical point in LES is for the user to ensure that the energy-bearing scales of the turbulent field are fully 
resolved by the numerics and grid spacing. This requirement is necessary for acceptable accuracy because the SGS model is only satisfactory when the cut-off wave number lies within the inertial subrange. The subgrid scale (SGS) model which is used to model the small scales unresolved by the numerical mesh is a very important point in LES. Most SGS stress models are based on an eddy viscosity assumption and one of the first and widely used models was suggested by Smagorinsky (1963). Based on this model a lot of new models have been developed, e.g., a dynamic model (DM) by Germano et al.(1991), a mixed dynamic model (MDM) by Zang et al. (1993), a two-parameter mixed dynamic model (TMDM) by Salvetti et al. (1997) among others. All these models have been successfully applied to the simulations of some particular flow field selected by the investigator. An important effect of subgrid-scale motion in the turbulent flow, is the so called backscatter phenomenon which can be captured by most dynamic models. However, these models suffer from numerical instabilities. Moreover, most of them were validated on wall-bounded flows, such as channel flow (Piomelli et al., 1991; Piomelli, 1999a) and lid-driven cavity flows (Zang, 1993). Thus, a stable subgrid-scale model which represents more accurately the backscatter of the turbulent energy and is more suitable for wake type flows is highly desirable.

Regarding LES of ship wake flows, the work of Dommermuth, et al. (1996; 1993) is the most relevant to the present study. In their study they considered a relatively small rectangular region $(0.25 \mathrm{~L} \times 1.0 \mathrm{~L} \times 0.25 \mathrm{~L}$ at axial, transverse, and vertical directions, respectively, L being the ship cord length) and initialized it with fully developed isotropic, homogeneous turbulence (IHT) superimposed on the measured (or calculated via RANS) flow field and perform LES of the flow development in that box. A forcing function (or a stirring force) is applied to the momentum equation to provide a prescribed turbulent dissipation rate. The dissipation rate can be prescribed according to RANS or empirical information available. Periodic boundary conditions are applied in the axial direction. Free surface waves induced 
by turbulence are included in the LES computation in a semi-empirical manner while the disturbances due to Kelvin waves are subtracted from the velocity field and calculated separately using potential flow theory. These transient results in the box are projected onto the spatial domain to describe the flow development in the far wake by assuming that the temporal behavior predicted in the prescribed region is related to the spatial behavior through a Galilean transformation. This means that a full similarity is assumed between the turbulence in the prescribed region and the corresponding reference region located at a distance; $x=U_{0} t$, where $U_{0}$ is the ship speed.

As it stands, their approach relies too heavily on the initialization using RANS results. Since, in its true sense, spatial development is not calculated but assumed to be related to temporal development, the initial turbulent flow field must play a dominant role in the subsequent development of the turbulence. By imposing the turbulent dissipation rate (as calculated from RANS) via a forcing function, this method becomes even more dependent on RANS. Moreover, it is not clear if the periodic boundary conditions in the axial direction where there is a significant flow development are appropriate for wake flows. Based on all these uncertainties, a new approach is taken in the present study in which the assumptions mentioned above are eliminated, and both the spatial and temporal development of the flow are calculated directly and simultaneously with appropriate boundary conditions.

Another issue concerning simulations of ship wake flows is how to deal with the complicated ship geometry. Normally, the ship should be included in the simulations because the boundary layer generated by the ship walls is the source of turbulence. However, the available computer resources are still not enough to satisfy the demand of LES of ship wakes including all the ship surface due to a prohibitively large number of grid nodes needed to capture fine flow structures in the thin wall boundary layer, including the viscous sublayer. Thus, it is desirable to have an efficient and robust method to start the computations excluding the body itself, if the focus is on the wake flow. The mean flow velocity as well 
as the turbulent quantities, such as turbulence intensity, time scale and length scale could be prescribed at the inflow boundary from empirical data or RANS (Reynolds Averaged Navier-Stokes) solution.

The principle function of LES is to resolve the large energy-bearing scales of the turbulent field. To achieve this target, very fine grids are essential. This becomes more evident for high Reynolds number flows such as ship wakes. However, the capability of even the fastest single processor computers today still can not satisfy the memory and CPU requirements of LES fully. Moreover, all the new supercomputers are built with multi-process architecture as shared or distributed memory. Workstations or PC clusters are constructed and used as an efficient and economical alternative. Parallel computing techniques make it possible to run large calculations by taking the advantage of the supercomputers or the so called "Beowolf" clusters. All this opens excellent opportunities for LES. In this regard an attempt was made in the present study to modify the serial code to run on a distributed memory cluster.

\subsection{Summary}

The objectives of this research are as follows:

1. Validate and improve an existing Navier-Stokes solver for LES applications.

2. Develop new or improved subgrid-scale models.

3. Validate and/or improve an approach to provide instantaneous turbulent inflow boundary conditions.

4. Investigate the physics of the turbulent ship wakes.

5. Parallelize the LES code using Message-Passing-Interface (MPI) technique. 


\section{Chapter 2}

\section{Mathematical Formulation}

In this chapter, the governing equations which describe the motions of an incompressible flow are presented. The Boussinesq approximation together with other simplifications are introduced to yield a reduced set of equations. Then increasing levels of complexity of the conservation laws are presented, and grid-filtered forms to describe the type of motions, which are resolved on a given grid, and finally with the curvilinear conservation laws, and discretized conservation laws in curvilinear coordinates are described.

\subsection{Governing Equations}

The application of the principles of conservation of mass and momentum to an incompressible, viscous fluid flow gives

Continuity:

$$
\frac{\partial u_{j}}{\partial x_{j}}=0
$$

Momentum:

$$
\frac{\partial u_{i}}{\partial t}+\frac{\partial\left(u_{j} u_{i}\right)}{\partial x_{j}}=-\frac{1}{\rho} \frac{\partial P}{\partial x_{i}}+\frac{\mu}{\rho} \frac{\partial^{2} u_{i}}{\partial x_{j}^{2}}+g \delta_{i 2}
$$


An additional equation is required to describe the conservation of a scalar, namely $\phi$ in this study.

$$
\frac{\partial \phi}{\partial t}+\frac{\partial\left(u_{j} \phi\right)}{\partial x_{j}}=\alpha \frac{\partial^{2} \phi}{\partial x_{j}^{2}}+S_{i}(\phi)
$$

Here $\alpha$ is a material coefficient which could be thermal diffusivity, conductivity, or viscosity, depending on which scalar equation is solved. $S_{i}(\phi)$ is a whole source/sink term.

The Einstein summation rule applies to repeated indices $(i, j=1,2,3)$ and $x_{1}, x_{2}$, and $x_{3}$ represent the three spatial axes in the Cartesian coordinate system. Note that the direction of gravity is in the negative $i=2$ direction. In the above equations, $u_{i}, P, \mu, t, g$ represent Cartesian velocity components, pressure, density, viscosity, time, the gravitational constant, respectively.

\subsection{Boussinesq Approximation}

The density of fluid may be written as

$$
\rho=\rho_{0}+\Delta \rho
$$

where $\rho_{0}$ is a reference density, and $\Delta \rho$ is the relative deviation from that reference state.

Substitution of Eqn. 2.4 into the momentum equation 2.2 gives

$$
\left(1+\frac{\Delta \rho}{\rho_{0}}\right)\left(\frac{\partial u_{i}}{\partial t}+\frac{\partial\left(u_{j} u_{i}\right)}{\partial x_{j}}\right)=-\frac{1}{\rho_{0}} \frac{\partial P}{\partial x_{i}}+\frac{\mu}{\rho_{0}} \frac{\partial^{2} u_{i}}{\partial x_{j}^{2}}+g \delta_{i 2}+\frac{\Delta \rho}{\rho_{0}} g \delta_{i} 2 .
$$

In the present research which focuses on the flow of ship wake the variation of density is very small compared to the reference density. Thus the Boussinesq Approximation can be applied in the momentum equation. By using the Boussinesq Approximation, the density 
variations in the inertial terms may be neglected while only in the buoyancy term that is retained. Let us redefine the pressure as

$$
p=\frac{1}{\rho_{0}}\left(P-\rho_{0} g x_{2}\right),
$$

the momentum equation can then be simplified as

$$
\frac{\partial u_{i}}{\partial t}+\frac{\partial\left(u_{j} u_{i}\right)}{\partial x_{j}}=-\frac{\partial p}{\partial x_{i}}+\nu \frac{\partial^{2} u_{i}}{\partial x_{j}^{2}}+\frac{\Delta \rho}{\rho_{0}} g \delta_{i} 2,
$$

where $\nu=\mu / \rho_{0}$ is the kinematic viscosity.

\subsection{Conservation Law Form}

In order to use a finite volume method for space integration (Ferziger and Peric, 1997), the above equations are expressed in a conservative form. To express these equations in a form more consistent, the equivalent, conservation law formulation is used by following the formulation of Zang et al. (1994):

$$
\begin{gathered}
\frac{\partial u_{j}}{\partial x_{j}}=0 \\
\frac{\partial u_{i}}{\partial t}+\frac{\partial F_{i j}}{\partial x_{j}}=S_{i} \\
\frac{\partial \phi}{\partial t}+\frac{\partial R_{j}}{\partial x_{j}}=S_{i}(\phi)
\end{gathered}
$$

Where

$$
F_{i j}=u_{i} u_{j}+p \delta_{i j}-\nu \frac{\partial u_{i}}{\partial x_{j}}
$$




$$
\begin{gathered}
S_{i}=\frac{\Delta \rho}{\rho_{0}} g \delta_{i} 2, \\
R_{j}=u_{j} \phi-\alpha \frac{\partial \phi}{\partial x_{j}} .
\end{gathered}
$$

Note that in order to calculate $S_{i}$ in Eqn.2.12, one more equation is required. One choice is to build a scalar equation for density directly (Zang, 1993), another choice is to solve the energy (temperature) equation and then get density from the temperature (Calhoun, 1998). At the current stage of this research, the variation of the density is ignored thus this term is set to zero. In the future when the density changes need to be considered, e.g. stratified flow, $S_{i}$ can be re-implemented together with a scalar equation for density. Another point needs to be made about $S_{i}(\phi)$. The actual expressing for $S_{i}$ really depends on the particular equation in which it appears. Any term that can not be included in the convection or diffusion terms may be included in this source term.

\subsection{Equations for the Resolved Motion}

The governing equations are discretized into algebraic form and solved on a numerical mesh (grid) system. In the case where the grid is relatively coarse or the turbulent energy is transferred through a wide range of spatial scales, there may be small-scale motions which the grid can not resolve. The scales which are resolved by the grid spacing are called the "resolved scales", while those which are beneath the grid's resolution are called the "subgrid scales".

For a generic flow variable $q$, the resolved component $\bar{q}$ and the subgrid-scale component $q^{\prime}$ are expressed as

$$
q=\bar{q}+q^{\prime}
$$


Mathematically, the resolved-scale field is obtained by applying the following spatial filtering operation to the original field

$$
\bar{q}\left(x_{1}, x_{2}, x_{3}\right)=\int_{D} \prod_{i=1}^{3} G_{i}\left(x_{i}, x_{i}^{\prime}\right) q\left(x_{i}^{\prime}\right) d x_{1}^{\prime} d x_{2}^{\prime} d x_{3}^{\prime}
$$

where $D$ denotes the calculation domain. In a finite volume or finite difference method, such as the one used in the present work, a box filter is usually used, in which $G_{i}=1, D$ spans only the local grid cell, and an appropriate scheme is used for the integration of $q$ within the cell. Applying the filtering operation to Eqns. 2.8 to 2.13, we obtain

$$
\begin{gathered}
\frac{\partial \bar{u}_{j}}{\partial x_{j}}=0 \\
\frac{\partial \bar{u}_{i}}{\partial t}+\frac{\partial \bar{F}_{i j}}{\partial x_{j}}=\bar{S}_{i}, \\
\frac{\partial \bar{\phi}}{\partial t}+\frac{\partial \bar{R}_{j}}{\partial x_{j}}=\bar{S}_{i}(\phi),
\end{gathered}
$$

where

$$
\begin{gathered}
\bar{F}_{i j}=\bar{u}_{i} \bar{u}_{j}+\bar{p} \delta_{i j}-\nu \frac{\partial \bar{u}_{i}}{\partial x_{j}}+\tau_{i j}, \\
\bar{S}_{i}=\frac{\bar{\Delta} \rho}{\rho_{0}} g \delta_{i} 2 \\
\bar{R}_{i}=\bar{u}_{j} \bar{\phi}-\alpha \frac{\partial \bar{\phi}}{\partial x_{j}}+\chi_{j} .
\end{gathered}
$$

Two extra terms which represent the effect of the subgrid-scale motion appear in the above equations: $\tau_{i j}$, which is called the subgrid-scale stress, and $\chi_{j}$, which is called the subgrid-scale flux. They are defined as 


$$
\begin{gathered}
\tau_{i j}=\overline{u_{i} u_{j}}-\bar{u}_{i} \bar{u}_{j} \\
\chi_{j}=\overline{u_{j} \phi}-\bar{u}_{j} \bar{\phi}
\end{gathered}
$$

The resolved field is represented by "overbar"ed quantities, and $\tau_{i j}$ and $\chi_{j}$ have to be modeled using the resolved quantities. In the present study, the models for $\tau_{i j}$ and $\chi_{j}$ are recasted to the following mixed form:

$$
\begin{gathered}
\tau_{i j}-\frac{\delta_{i j}}{3} \tau_{k k}=-2 \nu_{T} \bar{S}_{i j}+C_{1}\left(L_{i j}^{m}-\frac{\delta_{i j}}{3} L_{k k}^{m}\right), \\
\chi_{j}=-\alpha_{T} \frac{\partial \bar{\phi}}{\partial x_{j}}+C_{2} P_{j}
\end{gathered}
$$

where $\bar{S}_{i j}$ is the resolved strain rate tensor,

$$
\bar{S}_{i j}=\frac{1}{2}\left(\frac{\partial \bar{u}_{i}}{\partial x_{j}}+\frac{\partial \bar{u}_{j}}{\partial x_{i}}\right)
$$

$L_{i j}^{m}$, (the 'modified Leonard term', (Germano, 1986)), and $P_{j}$ are defined as

$$
\begin{gathered}
L_{i j}^{m}=\overline{\bar{u}_{i} \bar{u}_{j}}-\overline{\bar{u}}_{i} \overline{\bar{u}}_{j} \\
P_{j}=\overline{\bar{u}_{j} \bar{\phi}}-\overline{\bar{u}}_{i} \overline{\bar{\phi}}
\end{gathered}
$$

The model coefficients $C_{1}$ and $C_{2}$ can take the value 0 or 1 , or may be determined dynamically depending on the type of subgrid-scale model being used; $\nu_{T}$ and $\alpha_{T}$ are called the 'eddy viscosity' and the 'eddy diffusivity', respectively. In the computation, the isotropic parts $\tau_{k k}$ and $L_{k k}$ are lumped into the pressure gradient term. Rewriting $\tau_{i j}$ as 


$$
\tau_{i j}-\frac{\delta_{i j}}{3} \tau_{k k}=-\nu_{T} \frac{\partial \bar{u}_{i}}{\partial x_{j}}-\nu_{T} \frac{\partial \bar{u}_{j}}{\partial x_{i}}+C_{1}\left(L_{i j}^{m}-\frac{\delta_{i j}}{3} L_{k k}^{m}\right),
$$

one can see that the first term on the RHS of the above equation may be combined with the viscous term in $\bar{F}_{i j}$, which is the third term on the RHS of Eqn.2.19. Similarly, we may combine the first term on the RHS of Eqn. 2.25 with the diffusive term in $\bar{R}_{j}$, which is the second term on the RHS of Eqn.2.21. Substituting Eqns. 2.29 and 2.25 into Eq.2.16 to Eq. 2.21 and combining terms accordingly, we have

$$
\begin{gathered}
\frac{\partial \bar{u}_{j}}{\partial x_{j}}=0 \\
\frac{\partial \bar{u}_{i}}{\partial t}+\frac{\partial \bar{F}_{i j}}{\partial x_{j}}=\bar{S}_{i}, \\
\frac{\partial \bar{\phi}}{\partial t}+\frac{\partial \bar{R}_{j}}{\partial x_{j}}=\bar{S}_{T i}(\phi),
\end{gathered}
$$

where

$$
\begin{gathered}
\bar{F}_{i j}=\bar{u}_{i} \bar{u}_{j}+\bar{p} \delta_{i j}-\left(\nu+\nu_{T}\right) \frac{\partial \bar{u}_{i}}{\partial x_{j}} \\
\bar{S}_{i}=\frac{\bar{\Delta} \rho}{\rho_{0}} g \delta_{i} 2+\frac{\partial \nu_{T}}{\partial x_{j}} \frac{\partial \bar{u}_{j}}{\partial x_{i}}-C_{1} \frac{\partial L_{i j}^{m}}{\partial x_{j}}, \\
\bar{R}_{i}=\bar{u}_{j} \bar{\phi}-\left(\alpha+\alpha_{T}\right) \frac{\partial \bar{\phi}}{\partial x_{j}} \\
\bar{S}_{T i}(\phi)=\bar{S}_{i}(\phi)-C_{2} \frac{\partial P_{j}}{\partial x_{j}}
\end{gathered}
$$

Note, in Eqn.2.33, variable $p$ includes the isotropic part $\tau_{k k}$ and $L_{k k}$. 


\subsection{Coordinate Transformation}

To be able to treat problems of complex geometry, the above mentioned equations are transformed from the physical space to computational space and written for a generalized curvilinear coordinate system. In the following, the physical space is denoted by coordinates $\left(x_{1}, x_{2}, x_{3}\right)$, and the computational space by coordinates $\left(\xi_{1}, \xi_{2}, \xi_{3}\right)$. The density fluctuations in the RHS of the source term 2.34 in the momentum equation are neglected. By applying the chain rule of derivatives we have

$$
\frac{\partial}{\partial x_{j}}=\frac{\partial \xi_{m}}{\partial x_{j}} \frac{\partial}{\partial \xi_{m}}
$$

Using this we obtain the equations:

$$
\begin{gathered}
\frac{\partial \xi_{m}}{\partial x_{j}} \frac{\partial \bar{u}_{j}}{\partial \xi_{m}}=0 \\
\frac{\partial \bar{u}_{i}}{\partial t}+\frac{\partial \xi_{m}}{\partial x_{j}} \frac{\partial \bar{F}_{i j}}{\partial \xi_{m}}=\bar{S}_{i} \\
\frac{\partial \bar{\phi}}{\partial t}+\frac{\partial \xi_{m}}{\partial x_{j}} \frac{\partial \bar{R}_{j}}{\partial \xi_{m}}=\bar{S}_{T i}(\phi),
\end{gathered}
$$

where

$$
\begin{gathered}
\bar{F}_{i j}=\bar{u}_{i} \bar{u}_{j}+\bar{p} \delta_{i j}-\left(\nu+\nu_{T}\right) \frac{\partial \xi_{m}}{\partial x_{j}} \frac{\partial \bar{u}_{i}}{\partial \xi_{m}} \\
\bar{S}_{i}=\frac{\partial \xi_{m}}{\partial x_{j}} \frac{\partial \nu_{T}}{\partial \xi_{m}} \frac{\partial \xi_{n}}{\partial x_{j}} \frac{\partial \bar{u}_{j}}{\partial \xi_{n}}-C_{1} \frac{\partial \xi_{m}}{\partial x_{j}} \frac{\partial L_{i j}^{m}}{\partial \xi_{m}} \\
\bar{R}_{i}=\bar{u}_{j} \bar{\phi}-\left(\alpha+\alpha_{T}\right) \frac{\partial \xi_{m}}{\partial x_{j}} \frac{\partial \bar{\phi}}{\partial \xi_{m}} \\
\bar{S}_{T i}(\phi)=\bar{S}_{i}^{\prime}(\phi)-C_{2} \frac{\partial \xi_{m}}{\partial x_{j}} \frac{\partial P_{j}}{\partial \xi_{m}}
\end{gathered}
$$


$\bar{S}_{i}^{\prime}(\phi)$ in Eqn. 2.44 is the transformation of $\bar{S}_{i}(\phi)$ to the computational domain. In order to use the finite-volume discretization, it is desirable to recast the equations in 'StrongConservation-Law Form'. This is done by to multiplying the above equation by the inverse of the Jacobian defined as

$$
J^{-1}=\operatorname{det}\left(\frac{\partial x_{i}}{\partial \xi_{m}}\right)
$$

and utilizing the metric identity

$$
\frac{\partial}{\partial \xi_{m}}\left(J^{-1} \frac{\partial \xi_{m}}{\partial x_{i}}\right)=0
$$

to bring the term $J^{-1} \partial \xi_{m} / \partial x_{i}$ inside the derivative $\partial / \partial \xi_{m}$. By carrying out these operations and, in addition, defining the following

$$
\begin{aligned}
\bar{U}_{m} & =J^{-1} \frac{\partial \xi_{m}}{\partial x_{j}} \bar{u}_{j}, \\
G^{m n} & =J^{-1} \frac{\partial \xi_{m}}{\partial x_{j}} \frac{\partial \xi_{n}}{\partial x_{j}},
\end{aligned}
$$

we obtain the final set of governing equations

$$
\begin{gathered}
\frac{\partial \bar{U}_{m}}{\partial \xi_{m}}=0, \\
\frac{\partial J^{-1} \bar{u}_{i}}{\partial t}+\frac{\partial \bar{F}_{i m}}{\partial \xi_{m}}=\bar{S}_{i}, \\
\frac{\partial J^{-1} \bar{\phi}}{\partial t}+\frac{\partial \bar{R}_{m}}{\partial \xi_{m}}=\bar{S}_{T i}(\phi),
\end{gathered}
$$

where

$$
\bar{F}_{i m}=\bar{U}_{m} \bar{u}_{i}+J^{-1} \frac{\partial \xi_{m}}{\partial x_{i}} \bar{p}-\left(\nu+\nu_{T}\right) G^{m n} \frac{\partial \bar{u}_{i}}{\partial \xi_{n}}
$$




$$
\begin{gathered}
\bar{S}_{i}=J^{-1} \frac{\partial \xi_{m}}{\partial x_{j}} \frac{\partial \xi_{n}}{\partial x_{i}} \frac{\partial \nu_{T}}{\partial \xi_{m}} \frac{\partial \bar{u}_{j}}{\partial \xi_{n}}-C_{1} \frac{\partial}{\partial \xi_{m}}\left(J^{-1} \frac{\partial \xi_{m}}{\partial x_{j}} L_{i j}^{m}\right) \\
\bar{R}_{m}=\bar{U}_{m} \bar{\phi}-\left(\alpha+\alpha_{T}\right) G^{m n} \frac{\partial \bar{\phi}}{\partial \xi_{n}} \\
\bar{S}_{T i}(\phi)=\bar{S}_{i}^{\prime}(\phi)-C_{2} \frac{\partial}{\partial \xi_{m}}\left(J^{-1} \frac{\partial \xi_{m}}{\partial x_{j}} P_{j}\right) .
\end{gathered}
$$

In Eqn.2.55, $\bar{S}_{i}^{\prime}(\phi)$ should be changed accordingly, depending on which scalar equation is solved. In Eqns 2.47 and 2.48, $\bar{U}_{m}$ is the resolved volume flux (contravariant velocity multiplied by the inverse of the Jacobian or the volume of the computational cell) normal to the surface of constant $\xi_{m}$, and $G^{m n}$, which is called the 'mesh skewness tensor', measures the skewness of the grid cell. The 'contravariant velocity' is not a true velocity but is a quantity with the dimension $(1 / t)$. 


\section{Chapter 3}

\section{Numerical Method and Validation}

The nature of LES requires the use of accurate numerical schemes such that the numerical errors, and aliasing, etc, do not pollute the solution and mask the contribution of subgridscales. Theoretically, the higher the order of the numerical scheme, the better the resolution should be under the same grid spacing. However, the concept of "accuracy" should not be taken in the strict meaning of this word, especially the interpretation linked to the formal order of truncation error in the Taylor series expansion. It has been demonstrated by Rai and Moin (1991) that the higher order of accuracy combined with coarse grid spacing is not necessarily better(even the 7th order upwind scheme was found unsuitable for LES). Specifically, the higher order upwind biased finite difference methods damp a substantial amount of the higher wave number range due to numerical dissipation which also controls the aliasing. However, Jordan (1999) showed that severe damping or damping of the finer resolved scales can be minimized by improving the grid spacing. Rai and Moin recommend the central differencing (CD) schemes where the aliasing must be controlled by enforcing kinetic energy conservation. Higher order central differencing schemes have in addition the problem of artificial high frequency oscillations that may contaminate the turbulence field. Another fact that needs to be considered is that the accuracy of CD schemes deteriorate 
rapidly in the presence of non-uniform grids with large $(>3 \%)$ expansion ratio. Temporally, in LES, explicit schemes are preferable, but if stability is an issue, some implicitness, e.g. via Crank-Nicolson time splitting, can be introduced.

This chapter provides information on the numerical method and the validation of the methods described in the previous chapter. First a summary of references for the numerical methods and turbulence models used in this work are reviewed. Validations of these methods have been performed for four benchmarks, namely, channel flow, flow past square cylinder, $2 D$ mixing layer flow, and open channel flow. Comparisons between simulated and experimental results are made.

\subsection{Summary of Numerical Method}

The Navier-Stokes solver used in the present work was developed by Zang et al. (1994). Part of the pressure solver was based on the work of Perng (1990). A non-staggered grid is employed. The method used is essentially a finite volume formulation using two sets of variables: Cartesian variables defined at cell centers, and contravariant volume fluxes defined at cell faces. The governing equations are discretized in the general curvilinear coordinates. Time advancement is semi-implicit using an implicit Crank-Nicolson scheme for the diffusive and diagonal viscous terms and an explicit Adams-Bashforth scheme for the other terms. In the momentum equations, spatial derivatives are computed with secondorder central differences, except for the convective terms which can be discretized by either central difference (with special care due to numerical instabilities) or quadratic upwind scheme, a variation of the QUICK (Quadratic Upstream Interpolation for Convective Kinematics) scheme (Leonard, 1979). Convective terms in the scalar equation are discretised using the SHARP scheme (Leonard, 1988). Henceforth, in this study,unless specially emphasized, terms such as CD, QUICK, or SHARP scheme always refer to the discretization 
of the convective terms in the momentum equation or the scalar equation.

The fractional step approach (Kim and Moin, 1985) was used to for time advancement. The idea of the fractional step method is to split the momentum equation into two parts: the pressure vs. all the other terms. A three-step predictor-corrector solution procedure is then formulated such that: (1) a predicted velocity field is calculated which is not constrained by the continuity equation; (2) the pressure field is computed from the pressure Poisson equation; and (3) the true velocity field is obtained by correcting the predicted velocity with pressure. The pressure Poisson equation is solved with a multigrid method (Brandt, 1977), and the approximate factorization technique (Beam and Warming, 1976) is used in solving the momentum equation. For the details about all these methods the reader may refer to the above cited papers and Zang (1993).

\subsection{Subgrid Scale Model}

Spatially filtering the equations of motion introduces extra terms which are called the subgrid scale (SGS) stresses that must be modeled. These terms represent the effects of the subgrid-scale motion on the resolved motion; dissipate the forward scatter resolved energy or backscatter energy to the finest resolved scale motion (Mason and Thomson, 1992). In other words, the interaction of the resolved and modeled fields occurs mainly over a very narrow wave number range where the coarsest modeled scales interact only with the finest resolved scales. This is the basic hypothesis of the scale similarity or mixing model (Liu et al., 1994b) and it can been represented by Figure 3.1. There are several characteristics that make a reliable subgrid scale model. The most important is that it adequately dissipates the resolved turbulence energy. Those models which dissipate too much energy are called overactive models, whereas those which dissipate much less energy can be named underactive models (Fig.3.2). A second important feature is that the SGS stresses should 
vanish at the wall preferably with the correct asymptotic behavior. The SGS model should also turn off when no SGS dissipation should occur, i.e., in laminar, potential and fully resolved flow.

Smagorinsky's model (Smagorinsky, 1963) was developed by assuming that the small scales are in equilibrium so that energy production and dissipation are in balance. Recently, dynamic versions of Smagorinsky and mixed subgrid scale turbulence models have been developed, see for example, Germano et al. (1991), Zang et al. (1993), and Salvetti and Banerjee (1995). In these dynamic models, the essential characteristics mentioned above are relatively well satisfied (Piomelli, 1999a; Piomelli, 1998). In the present work, these SGS models have been tested in order to find out which model is more appropriate for wake type flows. However, the results obtained were not as good as expected. Thus it is desirable to seek a SGS model which may be more suitable for wake flow. Considering the many advantages, such as solving the kinetic energy explicitly, the one-equation SGS model is getting much more attention (Menon et al., 1996; Sohankar et al., 2000; Ghosal et al., 1995; Okamoto and Shima, 1999). In Chapter 5 (this study), the main features of one-equation model will be reviewed and a one equation model is proposed.

\subsection{LES of Channel Flow}

Turbulent channel flow has been studied widely using experimental measurements (Clark, 1968; Hussain and Reynolds, 1975), Reynolds-Averaged-Navier-Stokes (RANS) simulation (C.Wilcox, 1993), Direct Numerical Simulation (DNS) (Mansour et al., 1988; Abe et al., 2001), and LES (Moin and Kim, 1982). Thus it is a good benchmark due to the existence of abundant data bases. The geometry of the channel flow is shown in Figure 3.3. The Reynolds number, based on the half channel width, is 33000 . A $66 \times 66 \times 130$ grid with a non-uniform distribution in vertical direction is used. This grid is selected so that the 
grid distribution in the y-direction (vertical to the wall) is sufficient to resolve the viscous sublayer $\left(y^{+}<5\right)$ and in the z-direction (spanwise) is capable to capture the 'streaks' in the wall region (Moin and Kim, 1982). no-slip boundaries were used for the bottom and top walls with $y^{+} \cong 3.095$ at the first grid node, while the other boundaries are periodic. The grid number as well as the other setups are similar to those used by Moin, et al. The flow is driven by a fixed pressure gradient.

First the QUICK scheme without any SGS model was tested. It resulted in much higher turbulence intensities compared to the experimental measurements. It seems that the numerical diffusion inherent in this upwind scheme is not enough to balance the energy dissipated by the subgrid-scale motion for this relatively fine grid. Next the dynamic subgridscale eddy viscosity model and the dynamic mixing subgrid-scale model (Zang et al., 1993) with central differencing scheme were applied. Although Vreman, et al.(1997) pointed that in Zang's model the test filter is incorrect, it should not have significant influence on the results. The reason lies on that, theoretically, any test filter larger than the filter of the original equation can be used in dynamic model. While they have many advanced features such as resolving subgrid dissipation well, capable of capaturing backscatter energy, it has been found that they predict much lower turbulence intensities compared to the experimental measurement. This was also noticed by Vreman, et al.(1997). In the end,the Smagorinsky model with $C=0.05$ with a central differencing scheme was applied, which gave results much closer to the measurements. The wall damping was not included in this study because grid is relatively fine in the wall range. The mean flow, the turbulence intensity and the streamwise velocity contours are shown in Figure 3.5, Figure 3.6 and Figure 3.7, respectively.

Fig. 3.5 shows that the classical log-law profile is captured correctly. In Figure 3.6 the turbulence intensity is shown and compared to the experiment data. The turbulence intensity is obtained from the formula $\frac{\sqrt{\overline{u^{\prime 2}}}}{u_{\tau}}$, etc., where $u_{\tau}(\cong 0.05)$ is calculated from the 
pressure gradient. From Figure 3.6, it can been seen that the predicted rms-fluctuations are in good agreement with the experiments except that the location of maximum fluctuations is predicted farther away from the wall. The reason may lie in the fact that not sufficient grid was used near the wall although finer grids were located in this region, which precludes the resolution of the whole boundary layer.

Figure 3.7 shows the instantaneous streamwise velocity contours and spanwise vorticity contours at different times on a central $x-y$ plane. The flow is fully turbulent with the large scale structures well captured. The presence of the boundary layer and the experimentally observed ejection and sweep events can be observed near the wall. Particularly, the resolution of the well known bursting phenomenon in the boundary layer is noteworthy. Figures 3.7(b) and 3.7(c) which are for two different instantaneous with sufficient separate time show the dynamics of the unsteady turbulent motion of large eddies captured in the present simulations.

\subsection{LES of Flow Past Square Cylinder}

The case of flow past a square cylinder has been studied by both experimental measurements (Lyn et al., 1995) and numerical simulations (Rodi, 1997; Sohankar et al., 2000). The geometry is shown in Figure 3.4. The Reynolds number, based on the cylinder side length is 22000, which is the same as in Lyn et al.'s experiments. A nonuniform grid $194 \times 162 \times 18$ in streamwise, vertical and spanwise directions, respectively, was used. Such grid size was selected because more grids were needed to resolve the flow in $\mathrm{x}$ and $\mathrm{y}$ directions due to the significant changes of the flow field in these directions, while in the spanwise direction (z-direction) the flow can be assumed as homogeneous. Near the body surface relatively finer girds were applied. As to the boundary conditions, symmetry boundaries in the normal direction, inflow and outflow (Neumann) boundaries in the 
streamwise direction, periodic boundaries in the spanwise directions, as well as no-slip boundaries on the surface of the cylinder were used. In this study case there was concern more about on the wake of the body because the goal is eventually to study ship wakes.

Similar to the study of channel flow, different subgrid-scale models with different numerical schemes were applied. The results showed that the QUICK scheme without any SGS model gave better results for this case compared to the measurements by Lyn et al. (1995), while dynamic model and Smagorinsky model had much lower turbulence fluctuations, both in frequency and magnitude. It seems that these models dissipate more energy than expected with the relatively coarse grid applied.

However, all the methods used gave good results for the mean flow parameters, such as the Strouhal Number of vortex shedding and the mean velocity profile.

In this case an oscillatory flow with a period of about $0.56 \mathrm{~s}$ was obtained, which is close to that reported by Rodi(1997). The predicted Strouhal Number is 0.1335 as compared to the experimental value of 0.132 reported by Lyn et al. (1995).

The velocity variation along the center-line is illustrated in Figure 3.8. Here the instantaneous velocity profile at the central plane of the cylinder is compared to the measurements (Lyn et al., 1995). The body is placed at $x=0$. The $X$-axis is nondimensionized by $D$ (the length of one side of the square) and $Y$-axis is nondimensionized by $U_{0}$ (inflow velocity).

The distributions of the rms-velocity fluctuations $\overline{u^{\prime 2}}$ and $\overline{v^{\prime 2}}$ (including the contribution of the periodic oscillations) along the center-line are depicted in Figure 3.9 as compared with the measurements. In the very near wake, the results agree very well with the experimental measurements while in the far wake the predicted fluctuations are smaller. This may be because the grid size used in the far wake was much coarser. The spanwise vorticity $\left(\omega_{z}\right)$ contours and the streamlines are shown in Figure 3.10. This Figure gives vorticitycontours at the center of $X Y$ plane. One can clearly see the features of the vortex shedding. Large turbulence structures are well resolved. The energy spectrum at one point in the near 
wake ( $2 \mathrm{D}$ from the rear edge) is shown in Figure 3.11. The inertial range captured in the calculations is an indication of the validity of the LES.

\subsection{LES of Plane Mixing Layer}

The mixing layer flow simulations have been performed in order to study the bubble dynamics in a turbulence environment. The bubble dynamics (Smirnov et al., 2001a) is a different topic and will not be included in this study. Here focus will be only on the turbulent flow field.

This study is based on the experimental measurements (Lazaro and Lashears, 1992a; Lazaro and Lashears, 1992b; Rightley, 1995). The computational domain is sketched in Fig.3.12, which is sized as $0.55 m \times 0.2 m \times 0.2 m$ in $x, y$, and $z$ direction, respectively. The mixing layer is generated by two separate parallel flows with different incoming velocities. A thin flat plate, which is $0.15 m$ length, $0.003 m$ height, and $0.2 m$ width (the whole spanwise extent), is mounted in the middle of the inlet plane. The velocity of the lower half flow is $0.28 \mathrm{~m} / \mathrm{s}$, while the upper half value is $0.07 \mathrm{~m} / \mathrm{s}$. Bubbles are carried in from the lower half. In this study, a sinusoidal force, with the amplitude of five percent of the mean flow, is added on the vertical velocity component of the flow in the lower half. The grid used in this simulation was a $194 \times 66 \times 42$ uniform grid on all three dimensions. The cell size is $2.8 \mathrm{~mm} \times 3.0 \mathrm{~mm} \times 4.8 \mathrm{~mm}$.

As for the boundary conditions, uniform inflow is applied at the inlet plane, except at the lower half, where a sinusoidal force is imposed on the vertical velocity component. Free gradient boundary is used for outflow. Slip-wall boundary conditions are used at the top and bottom ( $y$ direction). In the spanwise $(z)$ direction, periodic boundary conditions are applied. At the surface of the flat plate, slip-wall boundary conditions are used. The reason of using slip-wall boundary conditions is that in this computation the effect of the 
mixing layer is dominant.

Central differencing (CD) scheme was applied on the discretization of the convection term. The SGS model used was the Smagorinsky model.

Because the volume fraction of the bubbles was kept very small, the coupling between the carrier flow and the bubbles was not considered. In other words, the flow behaves as there were no bubbles inside. This approximation is also supported by the research of Elghobashi and Truesdell (1993) in which they indicated that the influence of such low dispersed phase particles (bubbles) would be seen in the smallest scales, not in the large, energy-containing scales dominating the mean and rms velocity profiles. Following the experiments (Rightley, 1995), the range of the computational domain is wholly confined to the developing region of the mixing layer.

The streamwise mean velocities and the fluctuations are shown in Fig. 3.13 at $x / \lambda=$ 1.25 , where $\lambda$ is the characteristic flow length scale and is equal to $8 \mathrm{~mm}$. The profile of the mean streamwise velocity is in good agreement with that of the measurements. However, near the top and the bottom regions $(y<-20 \mathrm{~mm}$ and $y>20 \mathrm{~mm}$ ), small differences exist. It is not clear, in the author's knowledge, why the measurements give a higher mean streamwise velocity at the lower half of the downstream location than the velocity at the inlet, which is $0.28 \mathrm{~m} / \mathrm{s}$. Theoretically, at a downstream station, after the fluid in the lower half is mixed with that of the upper half, which has a lower velocity $(0.07 \mathrm{~m} / \mathrm{s})$, the flow of the fluid in the lower half should expand. Thus the velocity should decrease according to satisfy the continuity, although the change is small. In the upper half, it seems that the flow is still in the earlier stages of development. The Kelvin-Helmholtz vortex is not strong enough to affect the flow. With the development of the mixing, at the downstream locations, such influence is remarkable and the flow is restrained at some extent. Then the streamwise velocity at the upper half will decrease.

The predicted rms velocity in axial direction is generally lower than the measurements. 
The reason may lie on the slip-wall boundary conditions applied on the flat plate, which means the influence of wall on the flow is removed. Thus leads to underestimation of turbulence intensities. Farther away from the region where the mixing occurs, the difference is even larger. Again, slip-wall boundary condition is used at the top and bottom boundaries and this may be the primary reason for the observed differences. Very little mixing occurs in these regions, so the turbulence intensity is almost zero. A similar phenomenon is observed for the vertical mean velocities and their fluctuations (Fig. 3.14).

The comparison of the predicted velocity field and the measurements at different axial locations are shown in Figs. 3.15 to 3.17. In the experiments, the velocity field is obtained by conditional averaging (phase averaging). Two periods of the conditional averages are presented here - one period being broken into ten phase bins. Each period corresponds to the characteristic length scale, $\lambda$, of the large coherent vortical structure. On the other hand, in the simulations, space averaging along the spanwise direction is carried out. Also in the simulations, a region of length $(2 \lambda)$ is depicted. The mid point of the depicted region corresponds to the location of the measurement. Although the averaging methods are not the same, it is still worthwhile to notice that the coherent vortical structures match very well. Besides, obviously, if the space averaging domain is transferred to the phase averaging domain, the measurements and simulations would match perfectly. The mean convective velocity has been subtracted in the Figures 3.15 to 3.17 .

The vertical velocity contours are shown in Fig. 3.18 in which the developing KelvinHelmholtz vortices are clearly observed. Correspondingly, the bubble distributions are presented in Fig. 3.19. The bubble cloud can be seen to be entrained by the fluid entering the mixing region from the high speed side into the cores of the coherent vortical structures present in the mixing region.

Nevertheless, based on previous work by others (Elghobashi and Truesdell, 1993; Truesdell and Elghobashi, 1994; Elghobashi and Lasheras, 1996), when there is a large density 
ratio between the phases, such as in this study, the influence of bubbles on the carrier phase may become important even though the void fraction remains small. Works on this topic are continuing under the current project.

\subsection{LES of Open Channel Flow}

The goal of this benchmark is to test the capability of the presently used code in representing the model of the free surface as the symmetry boundary. The computation was set up exactly the same as in channel flow in Section 3.3 except that half of the grid nodes in the spanwise wise direction were used in order to save the computational time. The top wall has been represented by the symmetry boundary conditions:

$$
\frac{\partial u}{\partial y}=0 \quad \frac{\partial w}{\partial y}=0 \quad v_{n}=-v_{n-1}
$$

Note, that $y$ represents the vertical direction, and $n$ and $n-1$ are the last two grid nodes in the vertical direction.

The computed mean streamwise velocity is shown in Fig. 3.20. It is seen that by using

the symmetry boundary condition a free surface type velocity profile could be obtained. This means that symmetry boundary conditions can be applied on the free surface. The streamwise velocity contours as well as the velocity vectors on the center vertical plane are shown in Fig.3.21. Clearly while the turbulence has been well captured near the wall, there is hardly turbulence near the free surface. The spanwise vorticity contours presented in Fig. 3.22 also confirm this conclusion. A wave-like flow field can be seen in Fig.3.21 although it is very weak. It is believed that this phenomenon was generated by the ejections and sweeps at the wall boundary layer. 


\subsection{Summary}

In this chapter, the solution method for the incompressible Navier-Stokes equations has been described briefly. The subgrid-scale models have been summarized and the necessity for refinement of the current SGS models has been emphasized. The approach has been tested by applying it to four benchmark cases: channel flow, flow past a square cylinder,

mixing layer flow, and open-channel flow. In general, good results have been achieved, testifying to the feasibility of the current LES approach. 


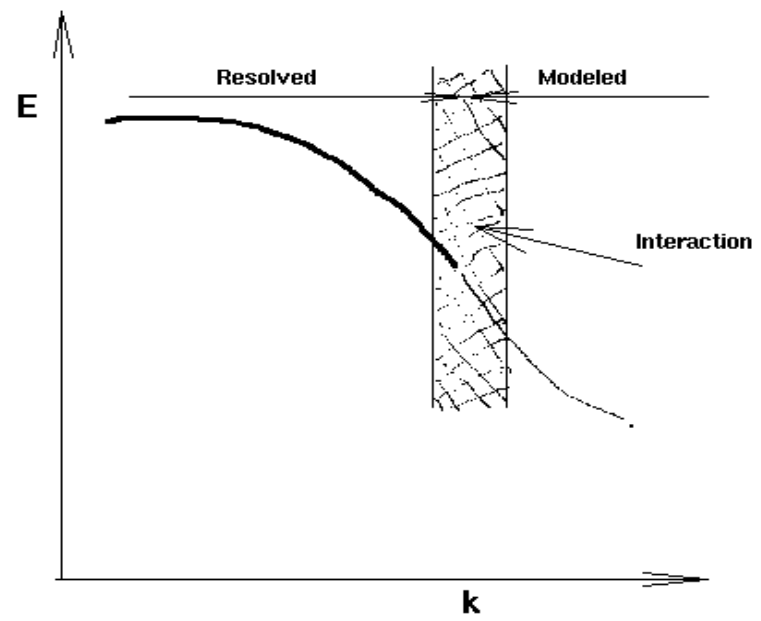

Figure 3.1: Resolved and modeled energy spectra

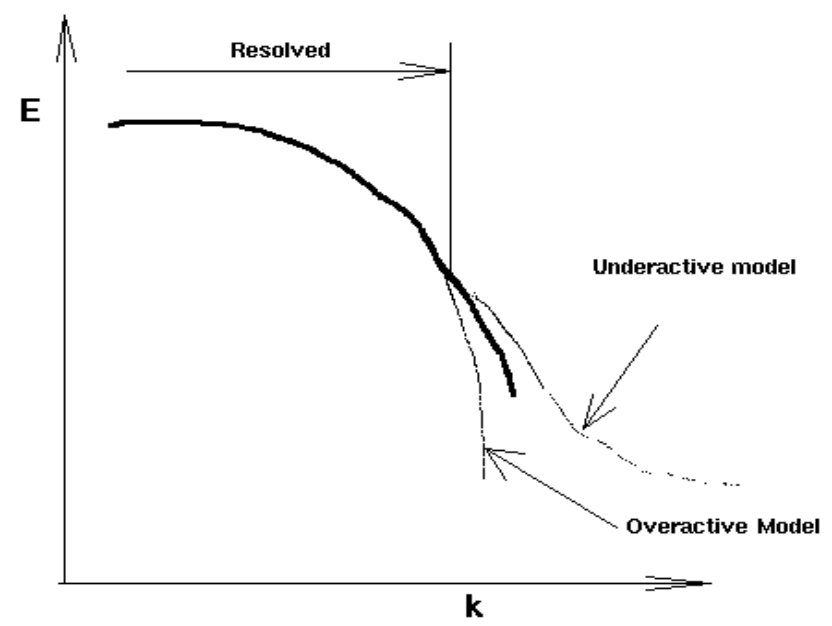

Figure 3.2: Overactive model and underactive model 


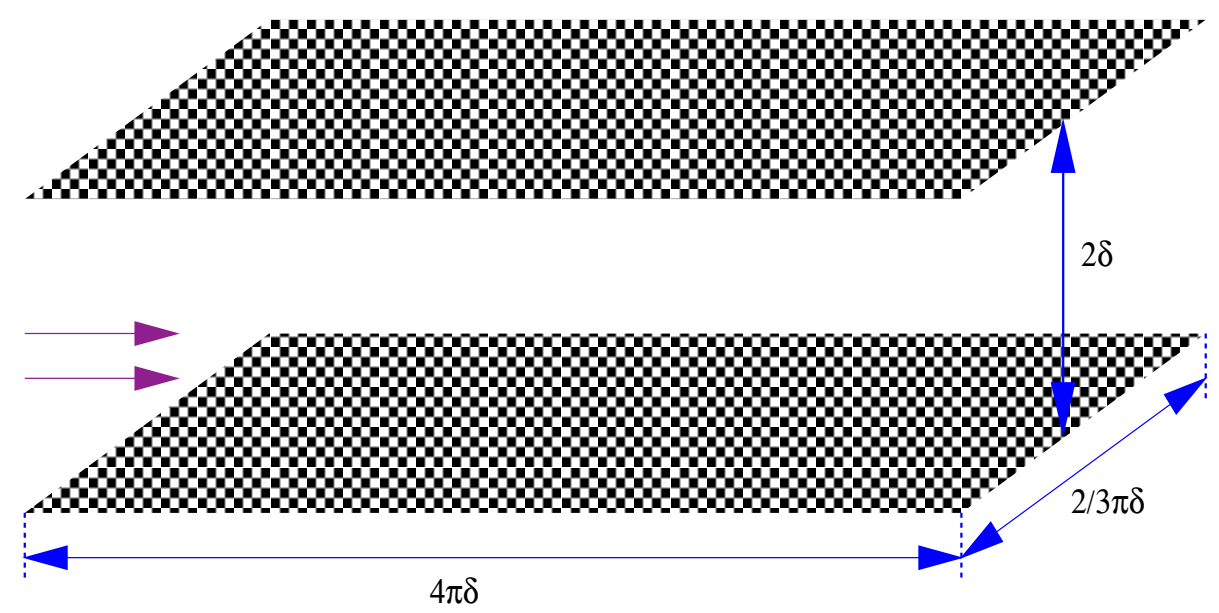

Figure 3.3: The geometry of the channel flow

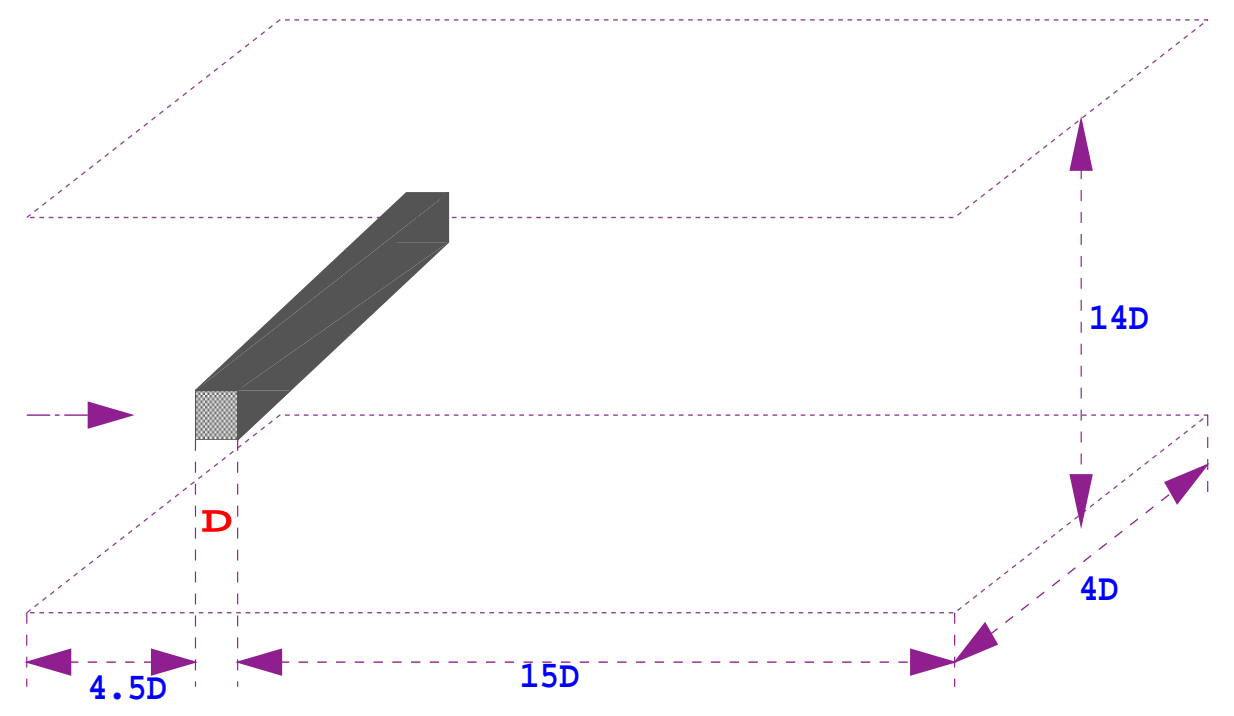

Figure 3.4: The geometry of the flow past a square cylinder 


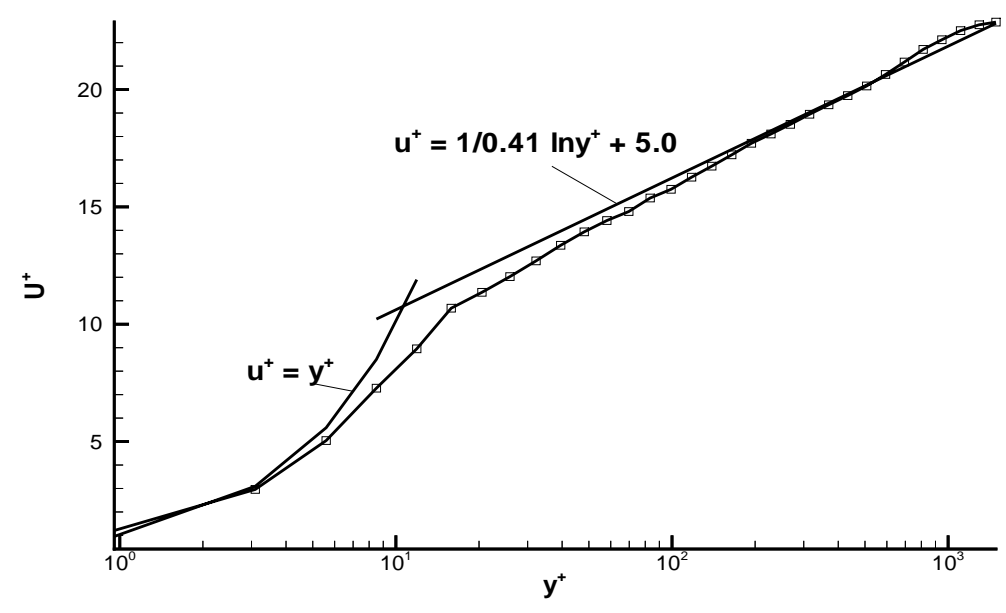

Figure 3.5: Mean flow logarithmic velocity profile in the channel flow (Central difference with the Smagorinsky model).

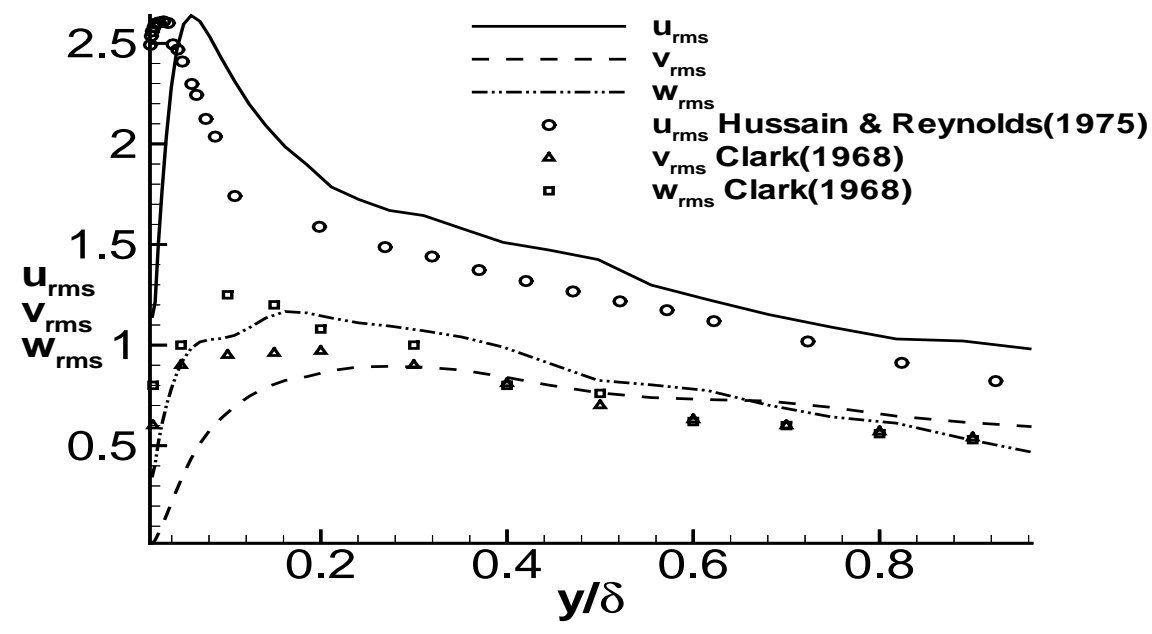

Figure 3.6: The turbulence intensity for the channel flow (central difference with the Smagorinsky model). Here $u_{r m s}=\sqrt{\overline{u^{\prime 2}}} / u_{\tau}$, similarly for $v_{r m s}$ and $w_{r m s}$. 


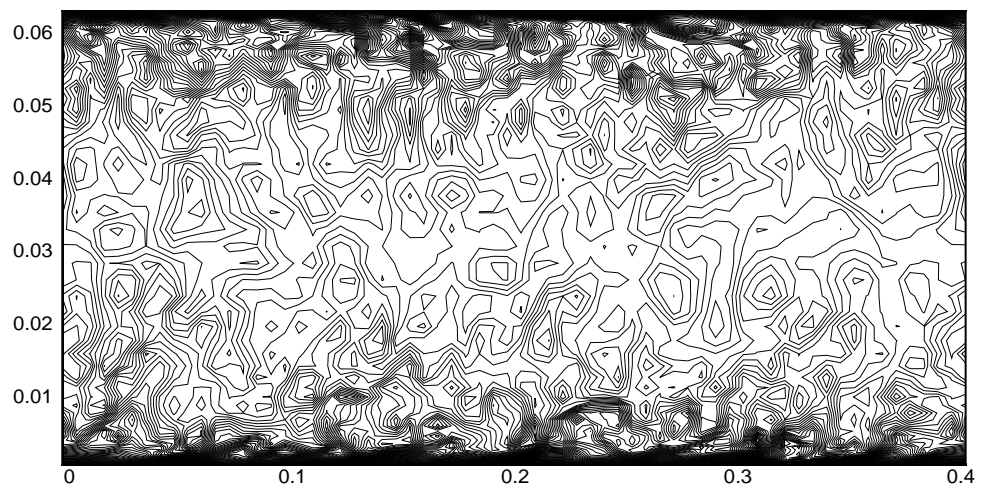

(a) u-contours

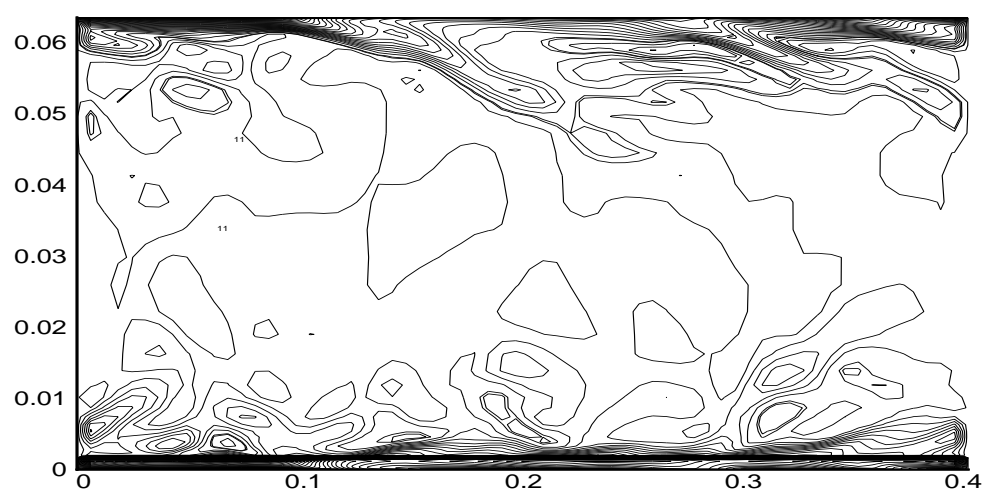

(b) contours of spanwise vorticity at Instant 1

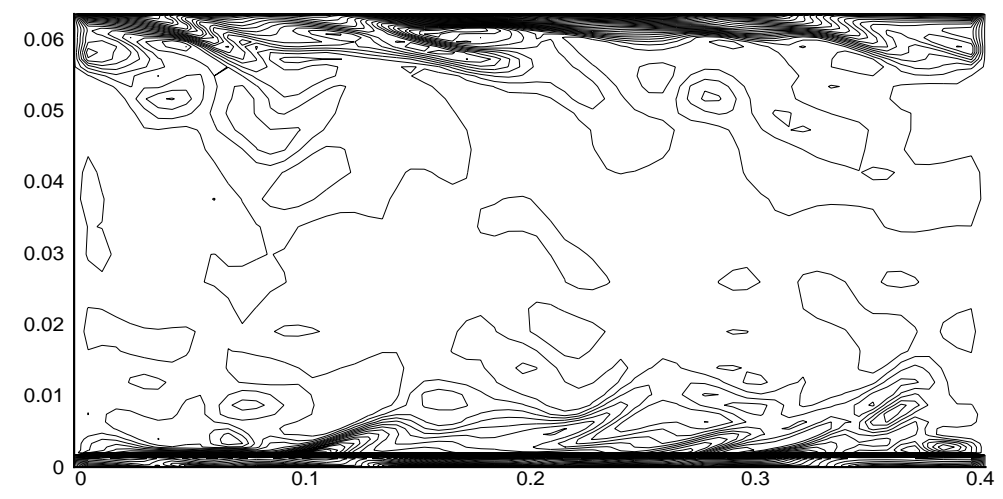

(c) contours of spanwise vorticity at Instant 2 (Sufficient time apart from Instant 1

Figure 3.7: Contours on the center x-y plane (Central difference with the Smagorinsky model) 


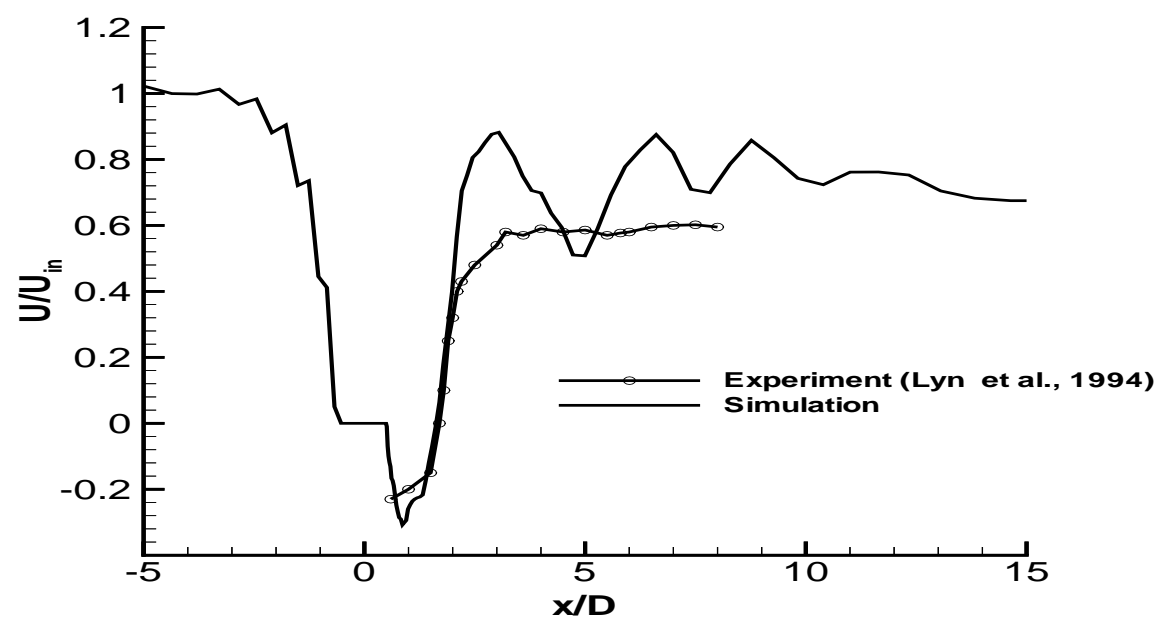

Figure 3.8: Mean velocity distribution on the center plane of the cylinder. The experiments are by Lyn et al. (1994) and the data starts behind the cylinder. The velocity decreases ahead of the cylinder because of the viscous forces. The cylinder is located in $-1 \leq$ $x / D \leq 1$. The QUICK scheme without any SGS model is used. 

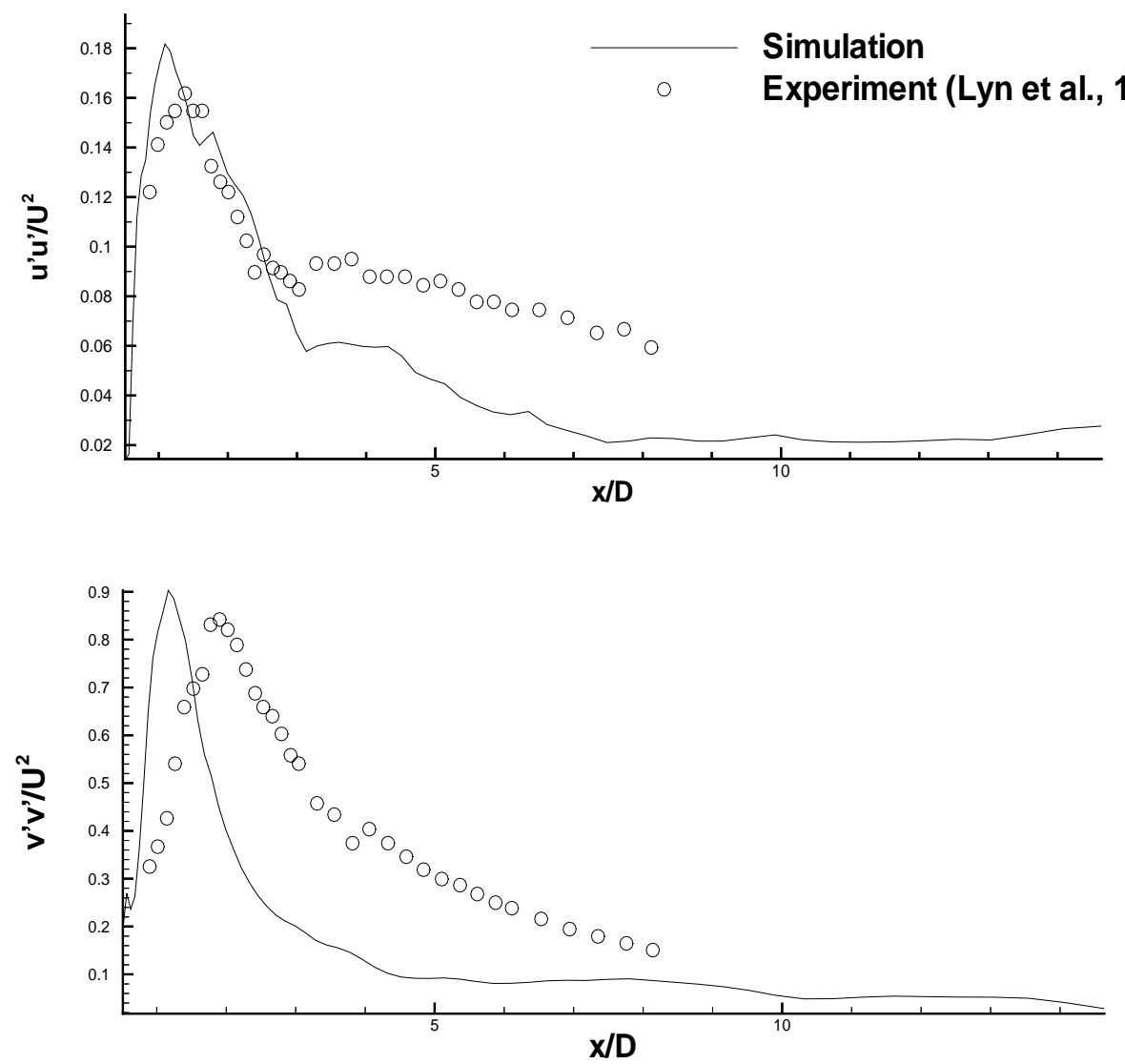

Figure 3.9: Turbulence intensity of the flow around the square cylinder. QUICK scheme without any SGS model is used. 


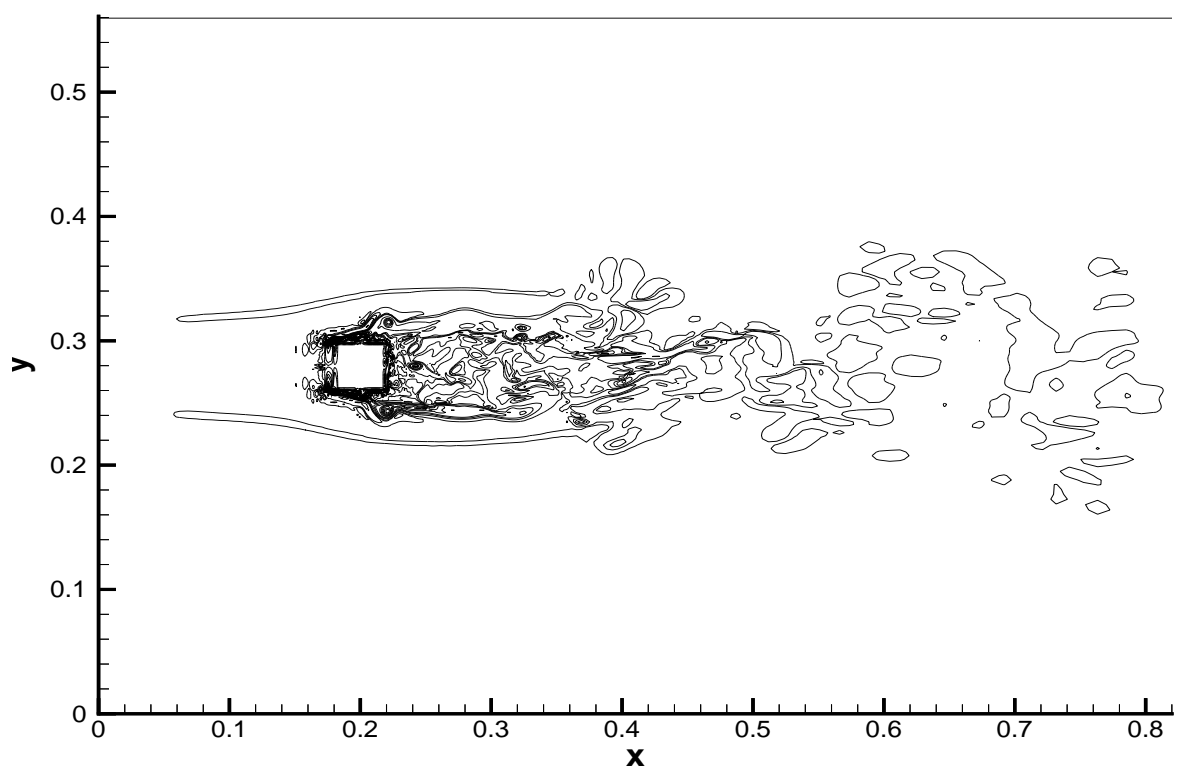

(a) $\omega_{z}$ - contours

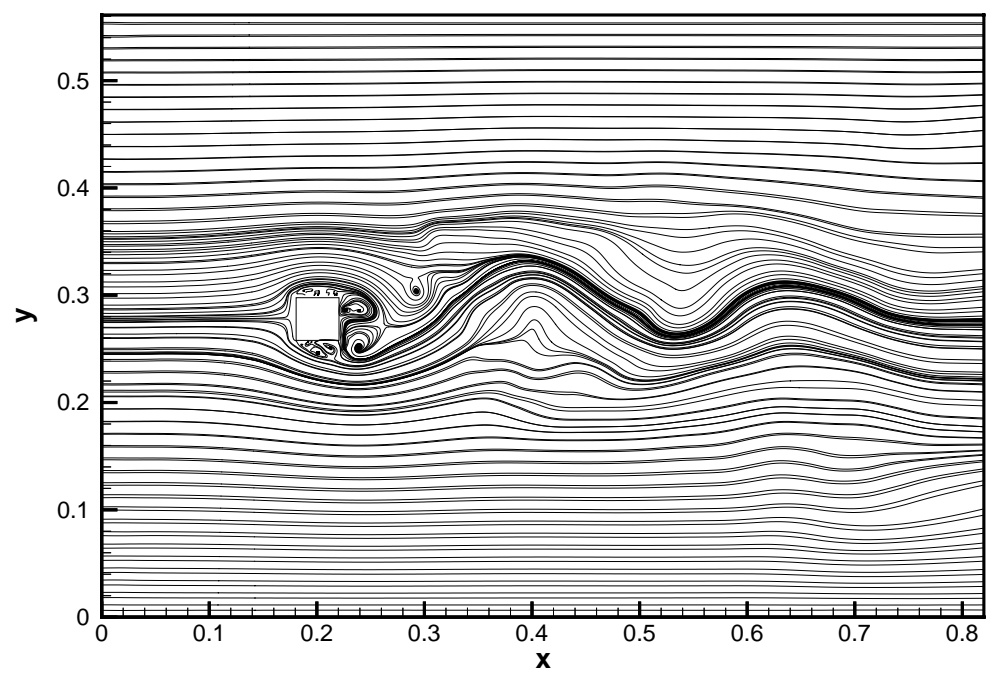

(b) streamlines

Figure 3.10: Contours of the spanwise vorticity and the streamlines along the center $x-y$ plane. QUICK scheme without any SGS model is used. 


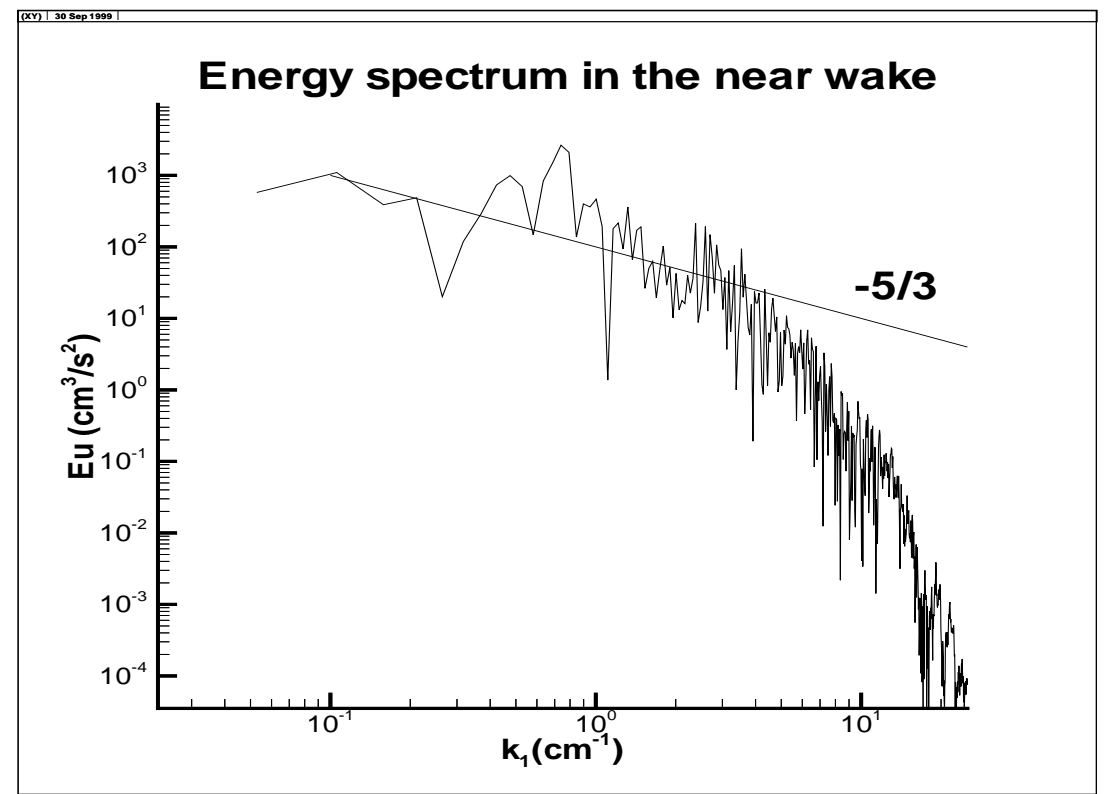

Figure 3.11: Energy spectrum in the near wake of the flow passed the square cylinder. QUICK scheme without any SGS model is used. 


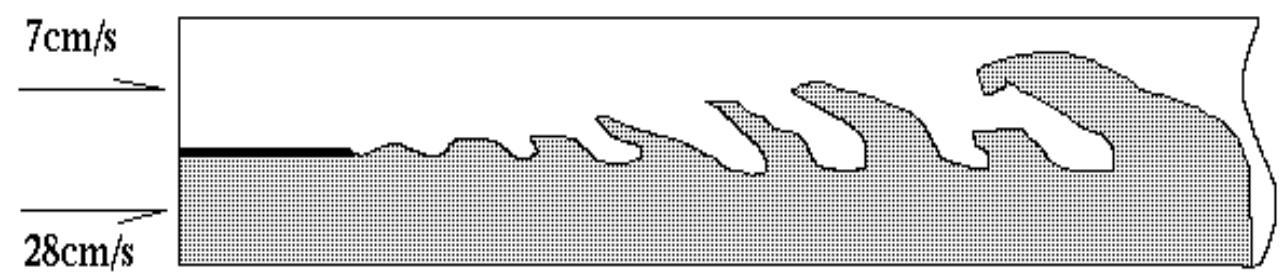

Figure 3.12: The sketch of plane mixing layer flow 


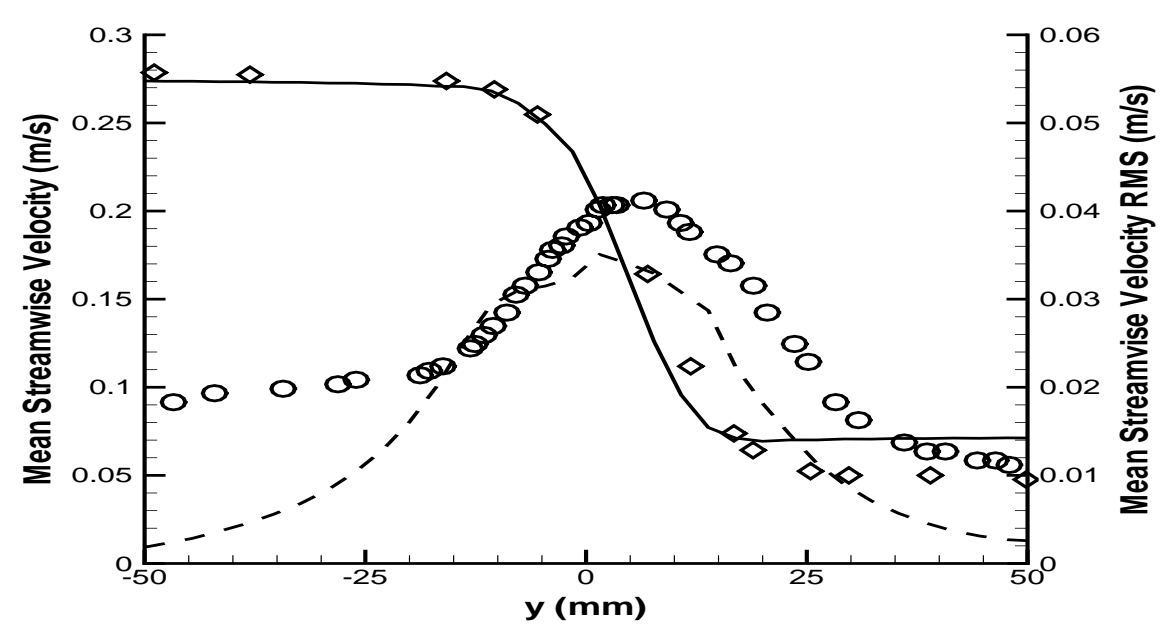

Figure 3.13: Mean and rms of streamwise velocity at $x / \lambda=1.25$. Symbols are from Rightley (1995): $\diamond:$ mean streamwise velocity; $\circ: \mathrm{rms}$ of streamwise velocity fluctuations. Lines: - - mean streamwise velocity of present simulation; $---: r m s$ of present simulation.

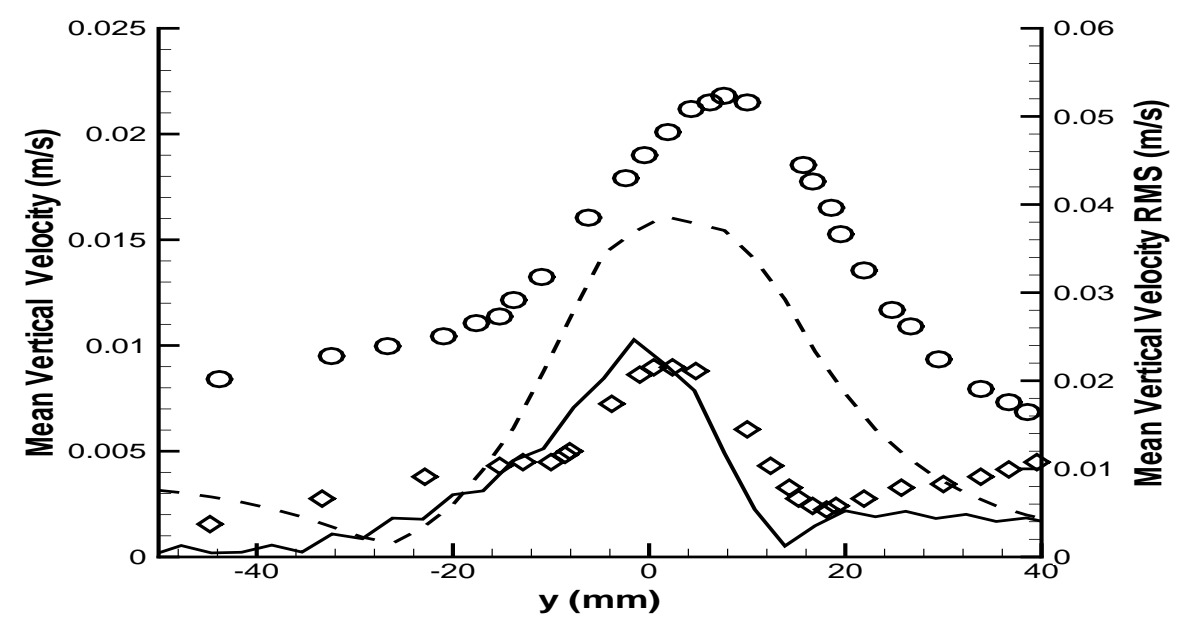

Figure 3.14: Mean and rms of vertical velocity at $x / \lambda=1.25$. Symbols are from Rightley (1995): $\diamond:$ mean vertical velocity; $\circ$ : rms of vertical velocity fluctuations. Lines: mean vertical velocity of present simulation; - - $:$ rms of present simulation. 


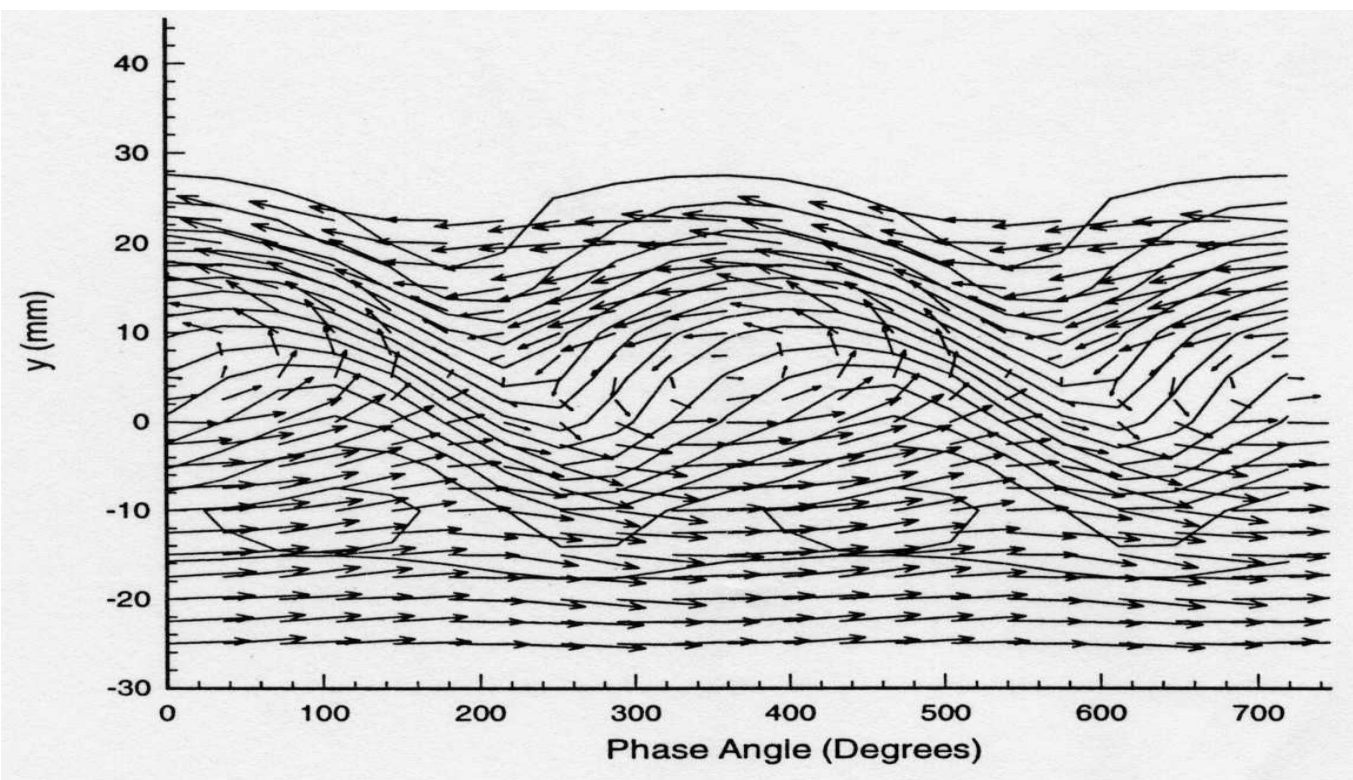

(a) Measurements by Rightley (1995)

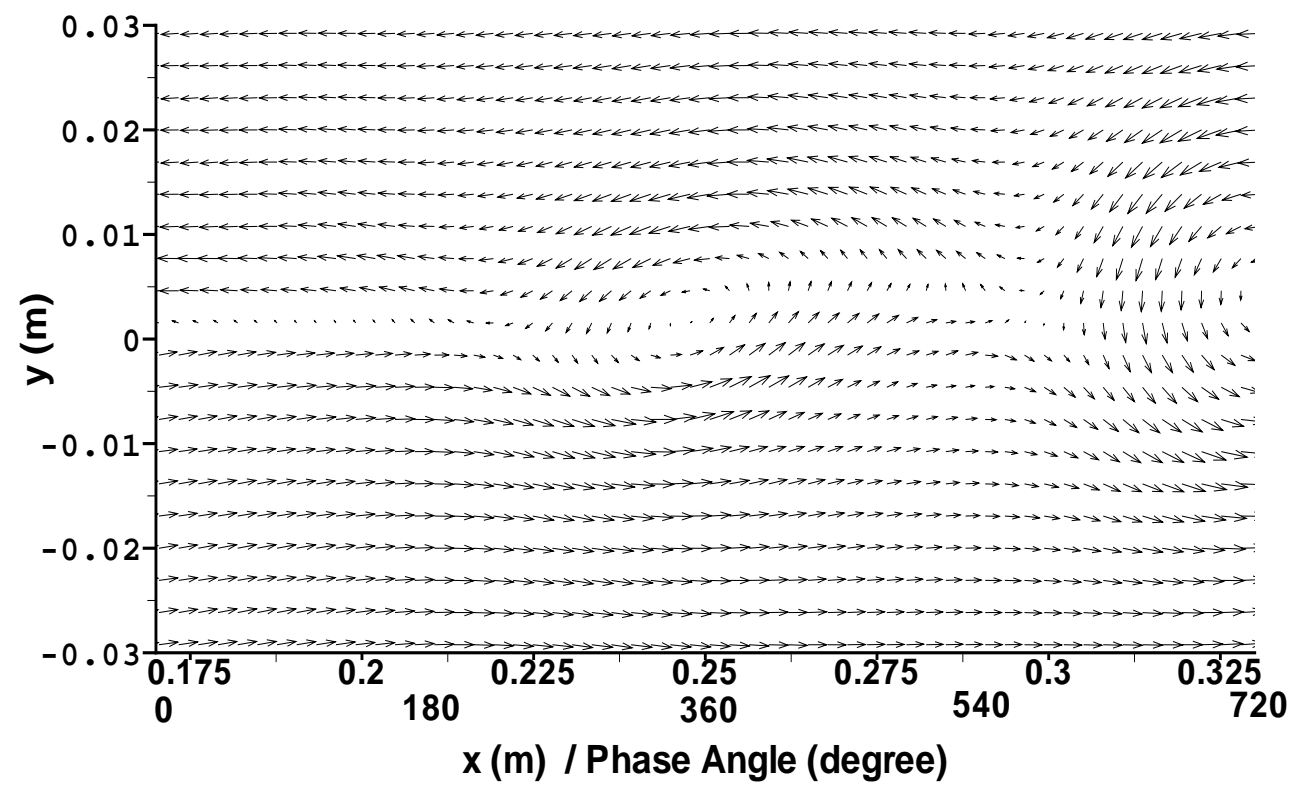

(b) Present simulations

Figure 3.15: Velocity vectors at $x / \lambda=1.25$ 


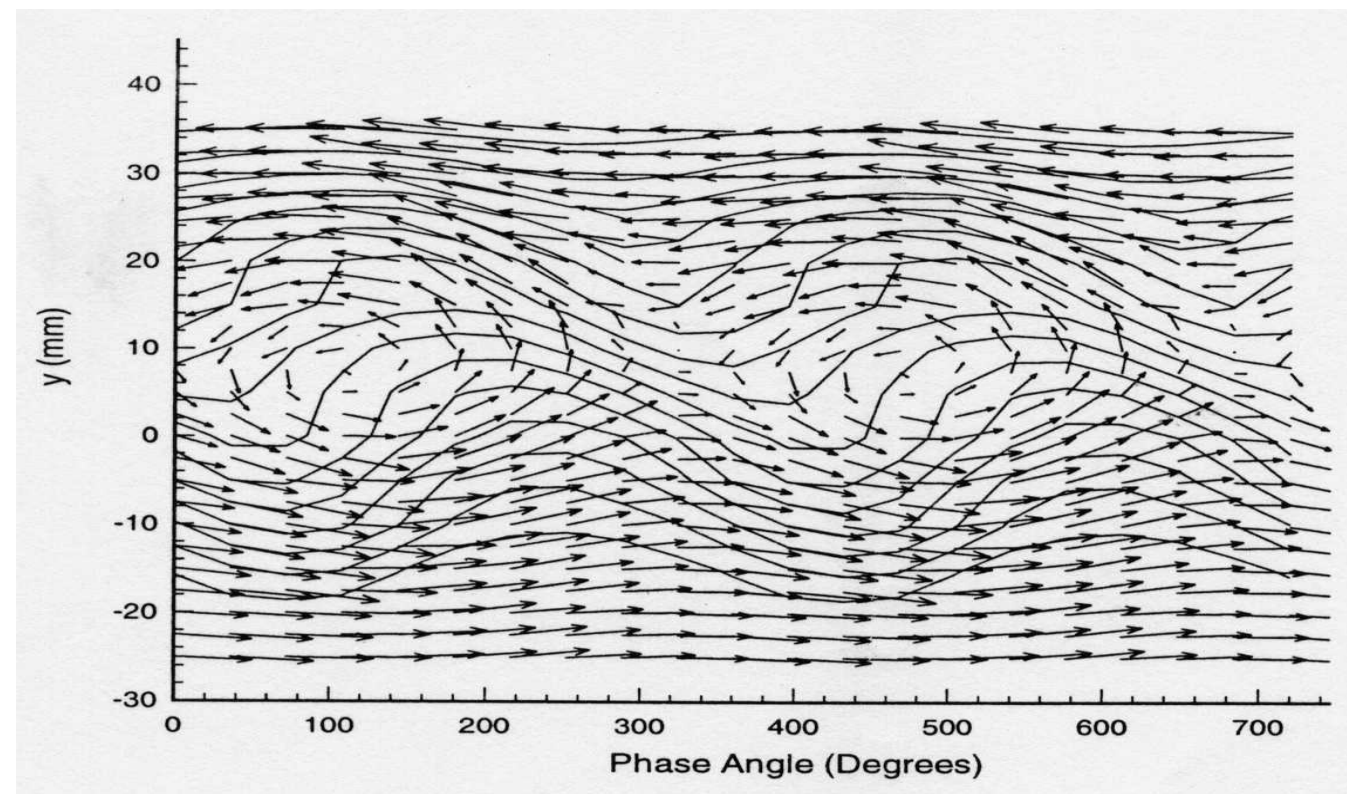

(a) Measurements by Rightley (1995)

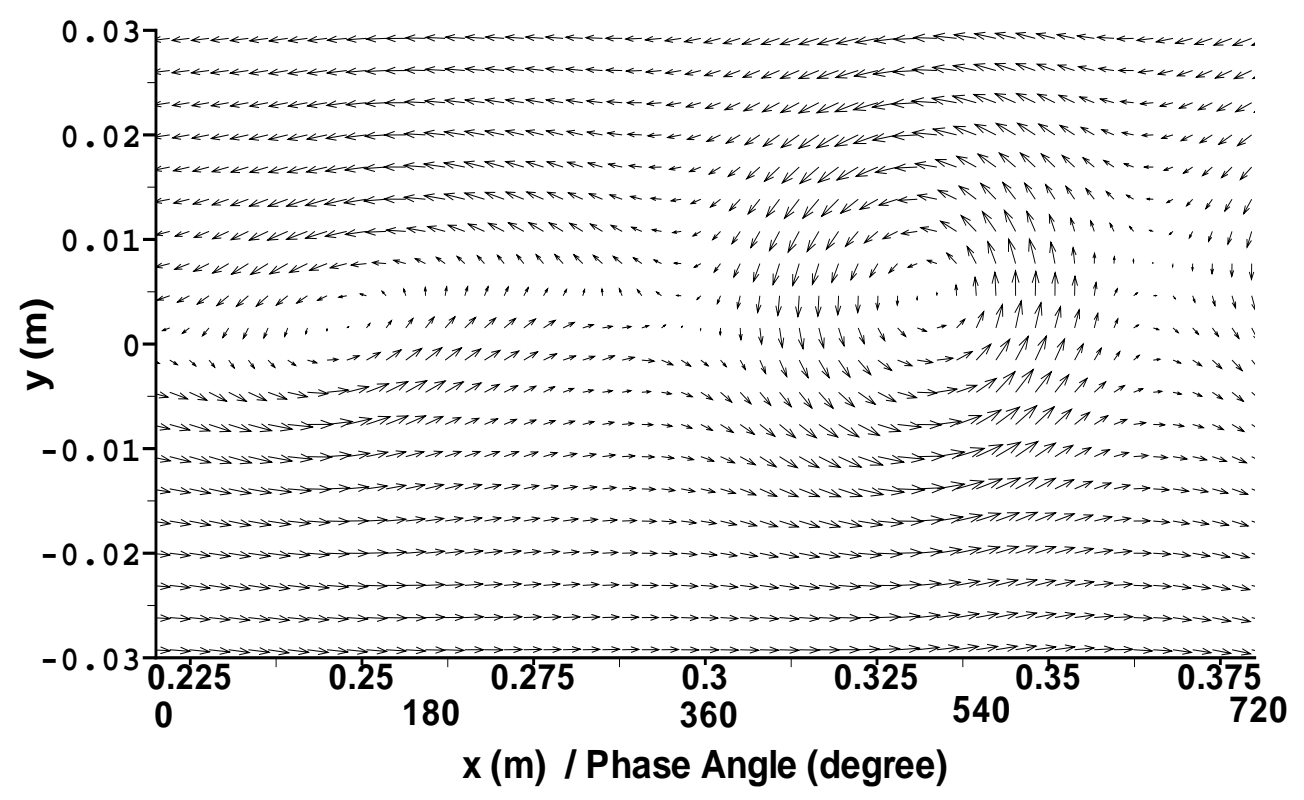

(b) Present simulations

Figure 3.16: Velocity vectors at $x / \lambda=1.88$ 


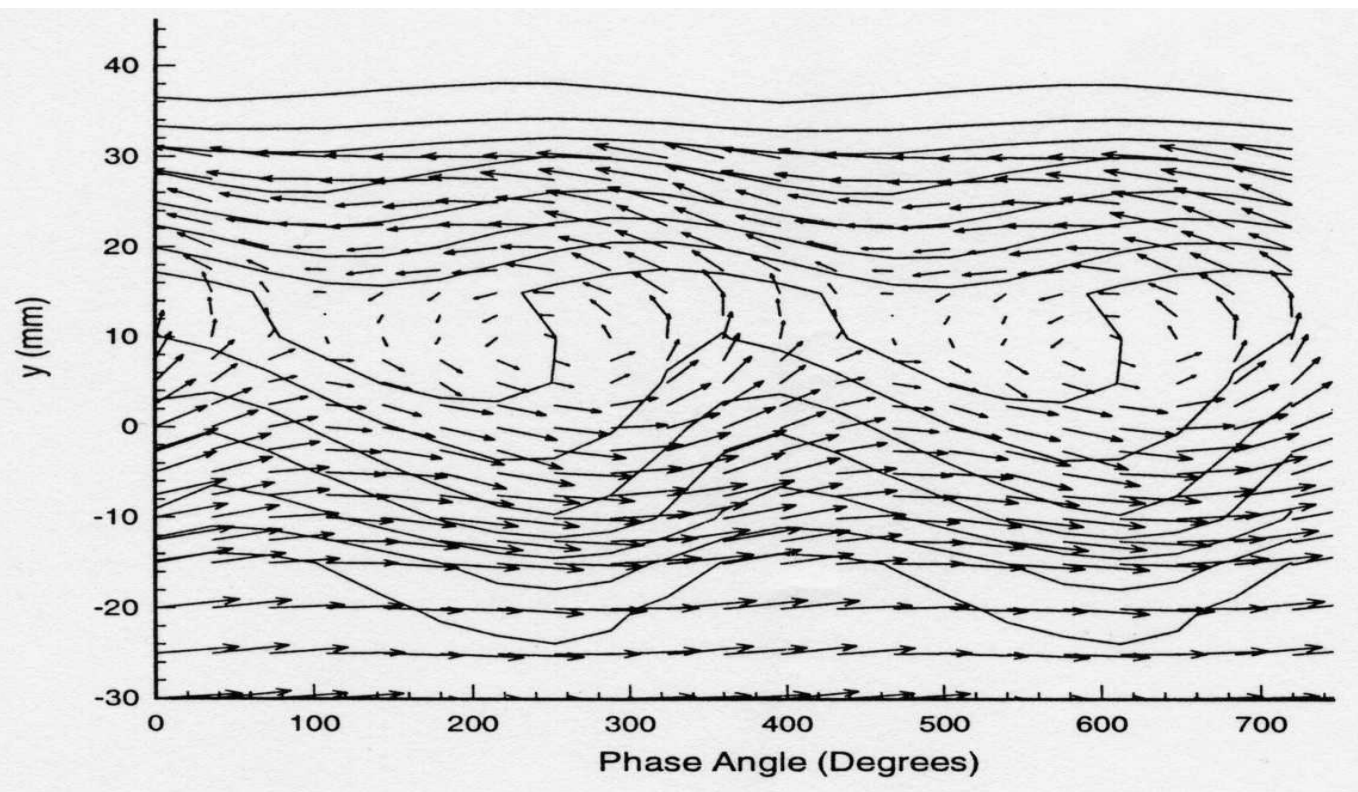

(a) Measurements by Rightley (1995)

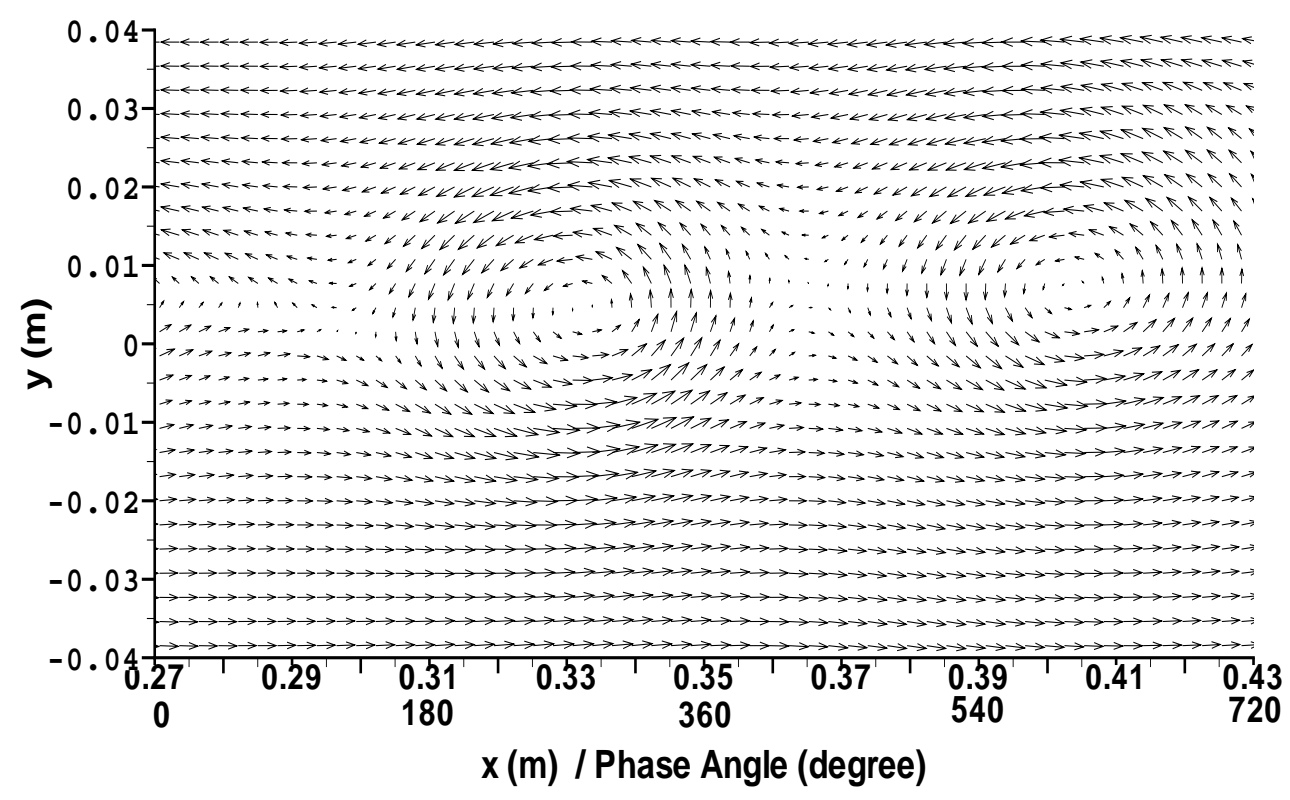

(b) Present simulations

Figure 3.17: Velocity vectors at $x / \lambda=2.50$ 


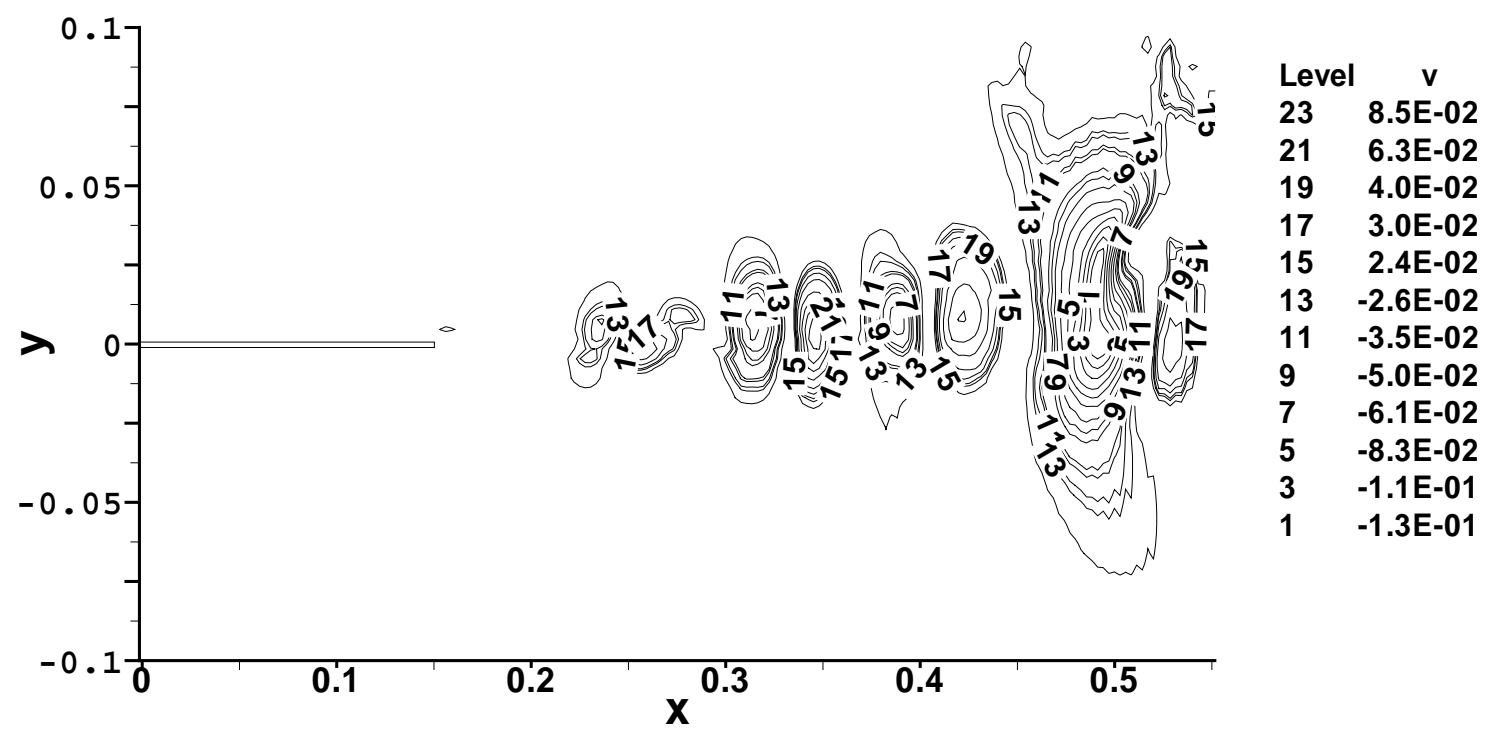

Figure 3.18: Vertical velocity contours

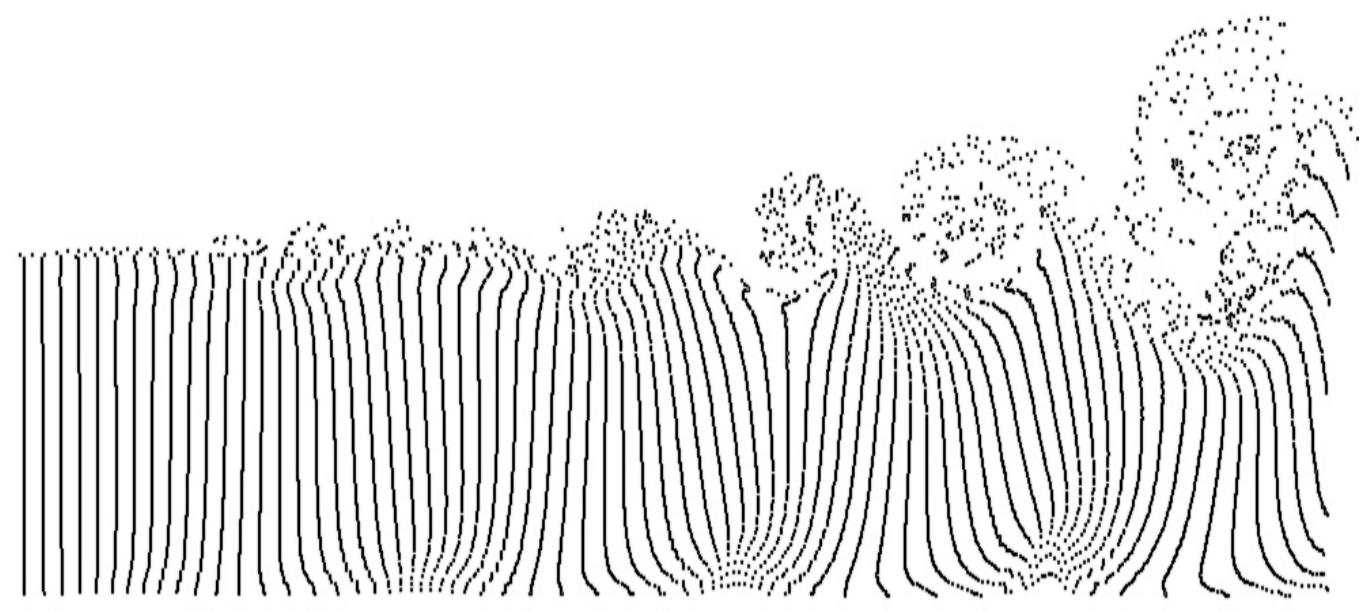

Figure 3.19: Bubble trajectories in the mixing layer, courtesy of Smirnov, A. (2001). 


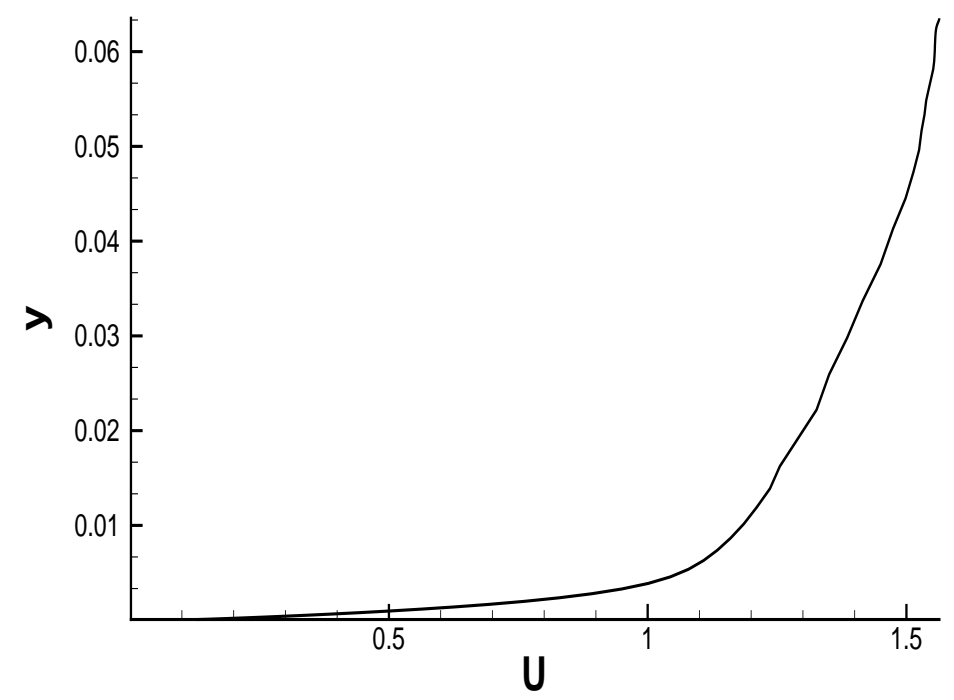

Figure 3.20: The mean streamwise velocity in the open channel flow

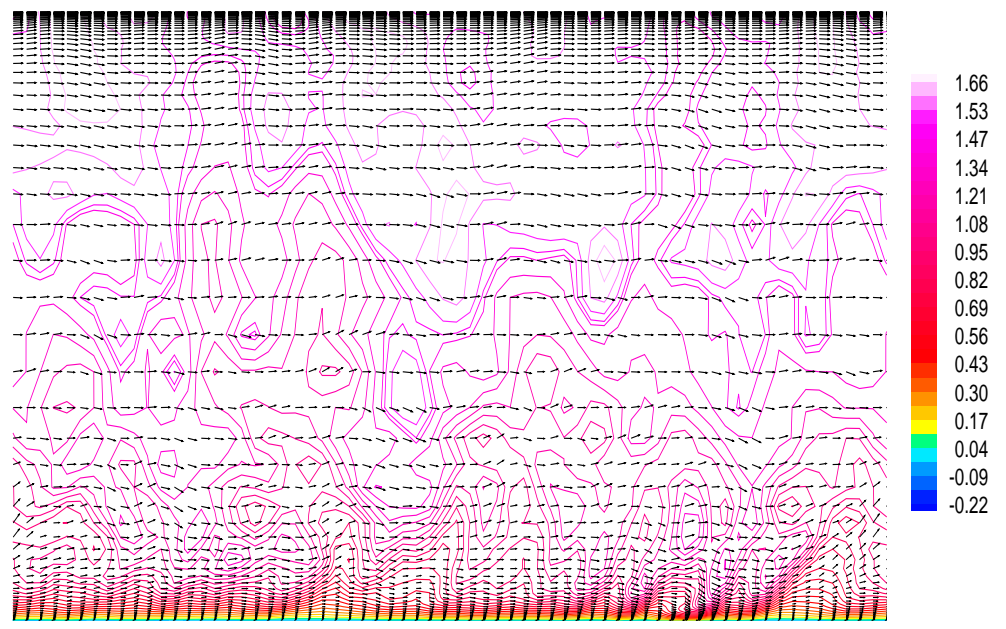

Figure 3.21: The contours of the instantaneous streamwise velocity and velocity vectors in the center vertical plane in the open channel flow 


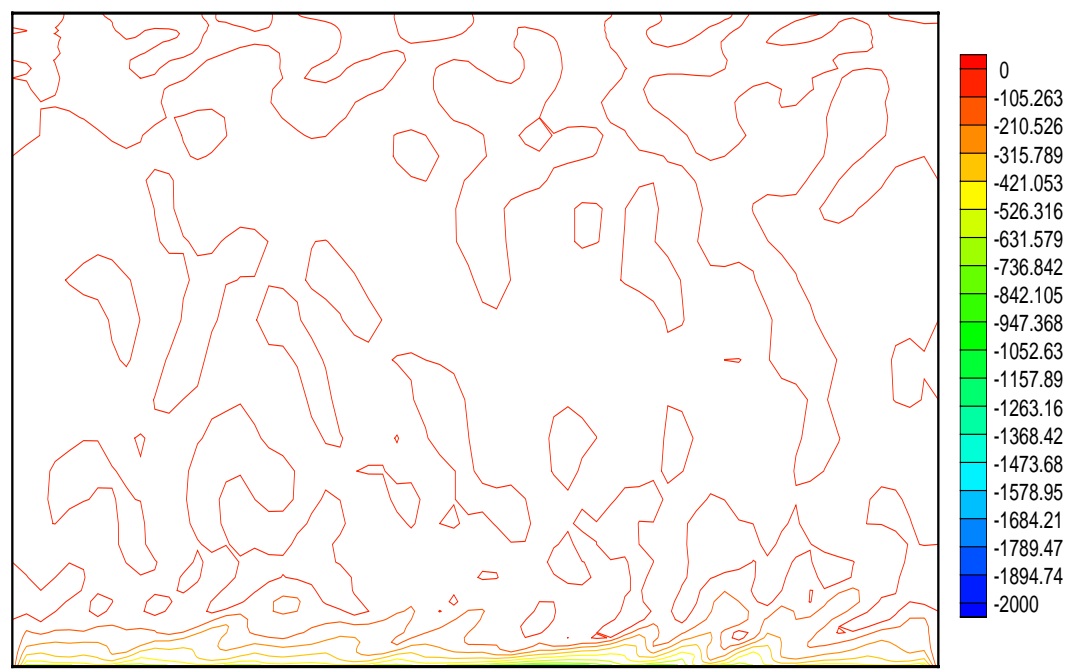

Figure 3.22: The contours of the instantaneous spanwise voticity in the center vertical plane in the open channel flow 


\section{Chapter 4}

\section{Random Flow Generation}

In this chapter, a Random Flow Generation (RFG) (Smirnov et al., 2000b; Shi et al., 2000b) approach is described that can provide turbulent initial or inlet flow conditions for LES. The technique is based on previous methods of synthesizing divergence-free vector fields from a sample of Fourier harmonics and allows the generation of a non-homogeneous anisotropic flow field representing turbulent velocity fluctuations. This approach has been verified by reproducing the turbulent channel flow.

\subsection{Introduction}

In a Reynolds Averaged Navier-Stokes (RANS) turbulence modeling approach information about turbulent fluctuations is contained in the time averaged Reynolds stresses of the form $\overline{u_{i} u_{j}}$. These are obtained as an outcome of a turbulence model that links Reynolds Stresses to mean flow quantities (e.g. k- $\epsilon$ model), or solves modeled transport equations for each Reynolds stress component (e.g. Reynolds Stress models). However, this is not the case when the large eddy simulation (LES) methodology is employed since the goal here is to 
explicitly resolve the large eddy turbulent fluctuations. In LES the inlet conditions can not be derived directly from experimental results, because of the unsteady and pseudo-random nature of the flow being resolved, unless, of course, the turbulent intensity is zero at the inlet, which is rarely the case. This problem becomes more important for spatially developing turbulent flows where for example the boundary or shear layer thickness changes rapidly. In such cases periodic boundary conditions can not be specified as in the case of a fully developed channel flow (Ravikanth and Pletcher, 2000). A similar situation exists when prescribing the initial conditions over the whole calculation domain. This can be of importance when the turbulent flow is not steady in the mean (i.e., non-stationary turbulence) and the transients of the flow are to be resolved. Even for stationary turbulent flows, if realistic initial conditions are not prescribed, the establishment of a fully developed turbulence takes an unreasonably long execution time. For these reasons it is necessary to initialize the flowfield with some realistic form of perturbation to provide the initial turbulent conditions. It is important that the perturbation be spatially correlated, as is the case with the real flow. For external flow problems the turbulent flow field can be initiated simply by appropriately perturbing the inlet flow-field. In this case an accurate representation of temporal correlations of the flow-field can be important. The inlet perturbation propagates throughout the domain and helps trigger the turbulence that is to be captured. One strategy is to begin the LES by initializing the flow field to that of a previously obtained RANS solution. A higher resolution grid is then used with an appropriate sub-grid-scale model. The Reynolds stress terms provided by the RANS solution can be used to construct spatially and temporally correlated perturbed inlet and initial conditions. In principle it is possible to predict turbulence via LES technique by starting from a quiescent flow or with the mean flow obtained from RANS. Unfortunately, it takes a very long time for a turbulent flow to develop spatially and temporally. This is especially true in the case of decaying turbulence in the absence of strong turbulence generating factors like walls. A reasonably accurate approach 
to this problem is used in modeling of boundary layer turbulence (Lund, 1998). It consists in applying a separate flow solver with periodic boundary conditions to construct the inlet conditions for the LES/DNS solver. It provides well-formed inflow conditions consistent with the solution of the Navier-Stokes equation, which makes it particularly suitable for DNS. However, its implementation may not be straightforward for the problems without well defined fully developed boundary/shear layers. For some engineering problems it may also be too expensive in the usage of computer resources and programming effort.

To remedy this problem the inlet and initial conditions are often viewed as consisting of a mean component and a randomly fluctuating component with the appropriate statistics. Most of the work done in this direction is based on simplified variants of a spectral method, in which Fourier harmonics are generated with the appropriate statistics and assembled into a random flow-field. Realistic turbulence spectra can be realized in this way. In the work of Lee et al. (1992) for example, a very good representation of turbulence spectra was achieved by using Fourier harmonics with a random phase shift. This is a rather efficient method to generate the inflow turbulence with pre-defined characteristics. However, it does not satisfy the continuity of the flow-field, which may be important in diminishing the size of the non-physical transition region between the inlet flow-field and the solution provided by the Navier-Stokes solver inside the computational domain.

A considerable amount of work in random flow generation has been performed in the area of particle dispersion modeling using the RANS approach (Zhou and Leschziner, 1991; Zhou and Leschziner, 1996; Li et al., 1994). RANS modeling produces smooth flow fields, which do not accurately disperse particles that are embedded in the flow. To correct this turbulent Reynolds stresses are used to generate temporally and spatially correlated fluctuations, such that the resultant instantaneous velocity can be superimposed on the particles to induce a realistic dispersion. A number of approaches found in the literature (Li et al., 1994; Bechara et al., 1994; Fung et al., 1992) are based on a variant 
of spectral method of generating an isotropic continuous flow-field proposed earlier by Kraichnan (1970). However, this flow-field does not satisfy the requirement of spatial inhomogeneity and anisotropy of turbulent shear stresses, which may be important in realistic flows. The method of Zhou and Leschziner (1991) complies with the latter requirement, but the resultant flow field does not satisfy the continuity condition and is spatially uncorrelated. For homogeneous isotropic turbulence, the initial conditions can also be constructed as described by Ferziger (1983). The approach is based on a vector curl operation and forward/backward Fourier transforms. The extension of this method to anisotropic inho-

mogeneous flows is not trivial. At least one study presents a successful application of Kraichnan's method to anisotropic flows (Maxey, 1987). The technique is based on filtering and scaling operations applied to the generated isotropic flow-field to filter only the vectors with the prescribed correlations. Again, the filtering operation may be expensive computationally. The method presented in this study is different in that it is based only on scaling and simple coordinate transformation operations.

It is the objective of this study to formulate a relatively simple random flow generation (RFG) algorithm, which can be used to prescribe inlet conditions as well as initial conditions for spatially developing inhomogeneous, anisotropic turbulent flows.

\subsection{Methodology}

To generate a more realistic flow field a modified version of Kraichnan's technique (Kraichnan, 1970) is used. The detailed information about this approach can be found from the references (Celik et al., 1999; Smirnov et al., 2000b). Here only a brief description is provided.

1. Given an anisotropic velocity correlation tensor 


$$
r_{i j} \equiv \overline{u_{i} u_{j}}
$$

of a turbulent flow field $\left\{\tilde{u}_{i}\left(x_{j}, t\right)\right\}_{i, j=1 . .3}$, find an orthogonal transformation tensor $a_{i j}$ that would diagonalize $r_{i j}{ }^{1}$

$$
\begin{array}{r}
a_{m i} a_{n j} r_{i j}=\delta_{m n} v_{(n)}^{\prime 2} \\
a_{i k} a_{k j}=\delta_{i j}
\end{array}
$$

As a result of this step both $a_{i j}$ and $v_{n}^{\prime}$ become known functions of space.

2. Generate a transient flow-field in a three-dimensional domain $\left\{v_{i}\left(x_{j}, t\right)\right\}_{i, j=1 . .3}$ using the new method based on Kraichnan (1970)

$$
\begin{aligned}
& v_{i}(\vec{x}, t)=\sqrt{\frac{2}{N}} \sum_{n=1}^{N}\left[p_{i}^{n} \cos \left(\tilde{k}_{j}^{n} \tilde{x}_{j}+\omega_{n} \tilde{t}\right)\right. \\
& \left.+q_{i}^{n} \sin \left(\tilde{k}_{j}^{n} \tilde{x}_{j}+\omega_{n} \tilde{t}\right)\right] \\
& \tilde{x}_{j}=\frac{x_{j}}{l}, \tilde{t}=\frac{t}{\tau}, v^{\prime}=\frac{l}{\tau}, \tilde{k}_{j}^{n}=k_{j}^{n} \frac{v^{\prime}}{v_{(j)}^{\prime}} \\
& p_{i}^{n}=\varepsilon_{i j m} \zeta_{j}^{(n)} k_{m}^{(n)}, q_{i}^{n}=\varepsilon_{i j m} \xi_{j}^{(n)} k_{m}^{(n)} \\
& \zeta_{i}^{n}, \xi_{i}^{n}, \omega_{n} \in N(0,1), k_{i}^{n} \in N(0,1 / 2),
\end{aligned}
$$

where $l, \tau$ are the length and time-scales of turbulence, $\varepsilon_{i j k}$ is the permutation tensor used in vector product operation (Spain, 1965), and $N(M, \sigma)$ is a normal distribution with mean $M$ and standard deviation $\sigma$. Numbers $k_{j}^{n}, \omega_{n}$ represent a sample of $n$ wavenumber vectors and frequencies of the modeled turbulence spectrum

\footnotetext{
${ }^{1} f_{, i} \equiv \frac{\partial f}{\partial x_{i}}$. Repeated indexes imply summation, parentheses around indexes preclude summation.
} 


$$
E(k)=16\left(\frac{2}{\pi}\right)^{1 / 2} k^{4} \exp \left(-2 k^{2}\right)
$$

3. Apply scaling and orthogonal transformations to the flow-field $v_{i}$ generated in the previous step to obtain a new flow-field $u_{i}$

$$
\begin{gathered}
w_{i}=v_{(i)}^{\prime} v_{(i)} \\
u_{i}=a_{i k} w_{k}
\end{gathered}
$$

The procedure above takes as an input the correlation tensor of the original flow-field $r_{i j}$ and information on length- and time-scales of turbulence $(l, \tau)$. As was shown in (Smirnov et al., 2000b; Shi et al., 2000c), the time dependent flow-field $u_{i}\left(x_{j}, t\right)$ generated by the procedure is divergence-free for homogeneous anisotropic flow fields and to a high-degree divergence free for inhomogeneous anisotropic fields. The shear-stresses of the generated flow-field are equal to $r_{i j}$. By virtue of Eq.(4.4), spatial and temporal variations of $u_{i}$ follow Gaussian distributions with characteristic length and time-scales of $l, \tau$; however, other distributions can be used to simulate different turbulence spectra.

This approach has been tested by applying it to isotropic turbulence, homogeneous anisotropic turbulence and inhomogeneous anisotropic turbulent flows (Celik et al., 1999; Smirnov et al., 2000a). The boundary layer is selected as a test case because it has all the features of non-homogeneous anisotropic turbulent flow. Experimental and large eddy simulation (LES) data exist for this flow field, providing both mean and fluctuating velocity profiles, as well as turbulent correlations. The details such as flow field, turbulence intensities $\left(u_{r m s}, v_{r m s}, w_{r m s}\right)$ are reproduced very well as compared with the experiments (Smirnov et al., 2001b). 


\subsection{Determination of the Length Scale and Time Scale}

To build the turbulent inflow boundary, embedding the right turbulence length scale and time scale is one of the crucial requirements. Generally, turbulence length scale can be obtained by using information of kinetic energy, $k$, and its dissipation rate, $\epsilon$,

$$
l=C_{\mu} \frac{k^{3 / 2}}{\epsilon}
$$

where $C_{\mu}$ is a constant.

However there is no effect of the filter or grid size, which is the baseline of LES. There are two feasible ways to account for the filtering effect.

1. Compare the length scale calculated from Eq. 4.10 and the filter, the grid size in this study, and use the larger one as the length scale in RFG. This is reasonable because in LES the resolved length scale of the turbulence can not be smaller than the size of the numerical mesh.

2. Use the length scale calculated from Eq. 4.10 in RFG directly, filter the resulting fluctuations, and then superimpose the filtered fluctuations on the mean parameters.

The turbulence velocity scale, $u$, can be obtained from the turbulent kinetic energy, $k$.

$$
u=\sqrt{\frac{2}{3} k}
$$

The turbulence time scale $\tau$ is computed by using

$$
\tau=l / u,
$$

or

$$
\tau=1 / \omega=C_{\mu} k / \omega,
$$


where $\omega$ is the so-called specific dissipation rate (C.Wilcox, 1993).

\subsection{Validation of RFG}

A turbulent channel flow case was used to test the reconstruction capability of RFG approach. Instead of solving the Navier-Stokes equation, the flow field is built by superimposing a RFG field on the given mean flow field. The purpose of this test is to check how close the turbulence field from RFG can be compared with measurements or DNS results.

The data used in this study was taken from DNS results by Kim and Moin (1989). The Reynolds number based on the velocity at the center line and half of the height of the channel is 7890. The domain used in this study was selected as $2 \delta \times 2 \delta \times \delta$, where $\delta$ is half of the channel height. The grid size was $30 \times 97 \times 30$ in $x, y$, and $z$ direction, respectively. In the vertical direction $(y)$, nonuniform grid is used and it matches the grid of the database, while in the streamwise $(x)$ and spanwise $(z)$ direction uniform grid was used.

The length scale was obtained by using a similar formulation as in a generic mixing length model, i.e.

$$
l_{y}=\left\{\begin{array}{ll}
\kappa y & y \leq 0.21 \delta \\
c \delta & y>0.21 \delta
\end{array}\right\}
$$

where $\kappa=0.41$, and far from the wall $(>0.21 \delta)$ the length scale was taken constant which is equal to $c \delta$, where $c=0.09$. The resulted length scale should be bigger than the cell size, which is given by as $\Delta=\left(\Delta x_{1} \Delta x_{2} \Delta x_{3}\right)^{1 / 3}$.

$$
l=\max \left(l_{y}, \Delta\right)
$$

The velocity scale is deduced from the turbulent kinetic energy of DNS. 
The Fourier space was sampled with 1000 wave-numbers selected according to Eq. 4.4. Fig. 4.1 and Fig. 4.2 show examples of the snapshots of the flow field. The size of the captured turbulent eddies clearly indicate the length scale used in the calculation. Fig. 4.2 shows the velocity on a plane parallel to the wall. The captured length scales and time scales indicated that the turbulence is isotropic and homogeneous in the transverse direction.

To compare the RFG simulations with the DNS data, the velocity profile along a vertical line in the center of the axial plane was stored for each simulated realization of the flowfield. The profiles of ten thousands time realizations were then used to calculate the mean flow characteristics. Fig. 4.3 shows the mean axial velocity, while Fig. 4.4 shows the fluctuating components as well as the Reynolds shear stresses. As can be seen, the DNS data is well reproduced. The energy spectrum at a point near the wall is presented in Fig. 4.5. The integral of this curve (the area under the curve) is approximately $1 \times 10^{(-6)}$ and it matches the turbulence intensity. This means the fluctuations generated from RFG truly captured the energy-scales.

\subsection{Summary}

The RFG approach used to construct the instantaneous turbulent flow field for a given mean flow field is validated. This approach is used in the present study to provide the initial condition of turbulent flow or the turbulent inflow boundary conditions. This is important in simulations of developing wake flows because of the difficulty to incorporate the whole wake-generating bluff body in LES. The turbulence information needed in RFG can be obtained from measurements, or RANS.

The RFG method has been validated by reproducing the turbulent flow field of channel flow. Comparisons of the results between RFG and DNS show that they are extremely well 
matched. This demonstrates the potential of the RFG approach to enhance the capabilities of large eddy simulations in complex geometries, which are otherwise prohibitive due to the limited computer capabilities. The advantages of this approach is that it can generate a turbulent flow field given a limited information on inlet conditions, thus giving a way to study the complex flow dynamics. 

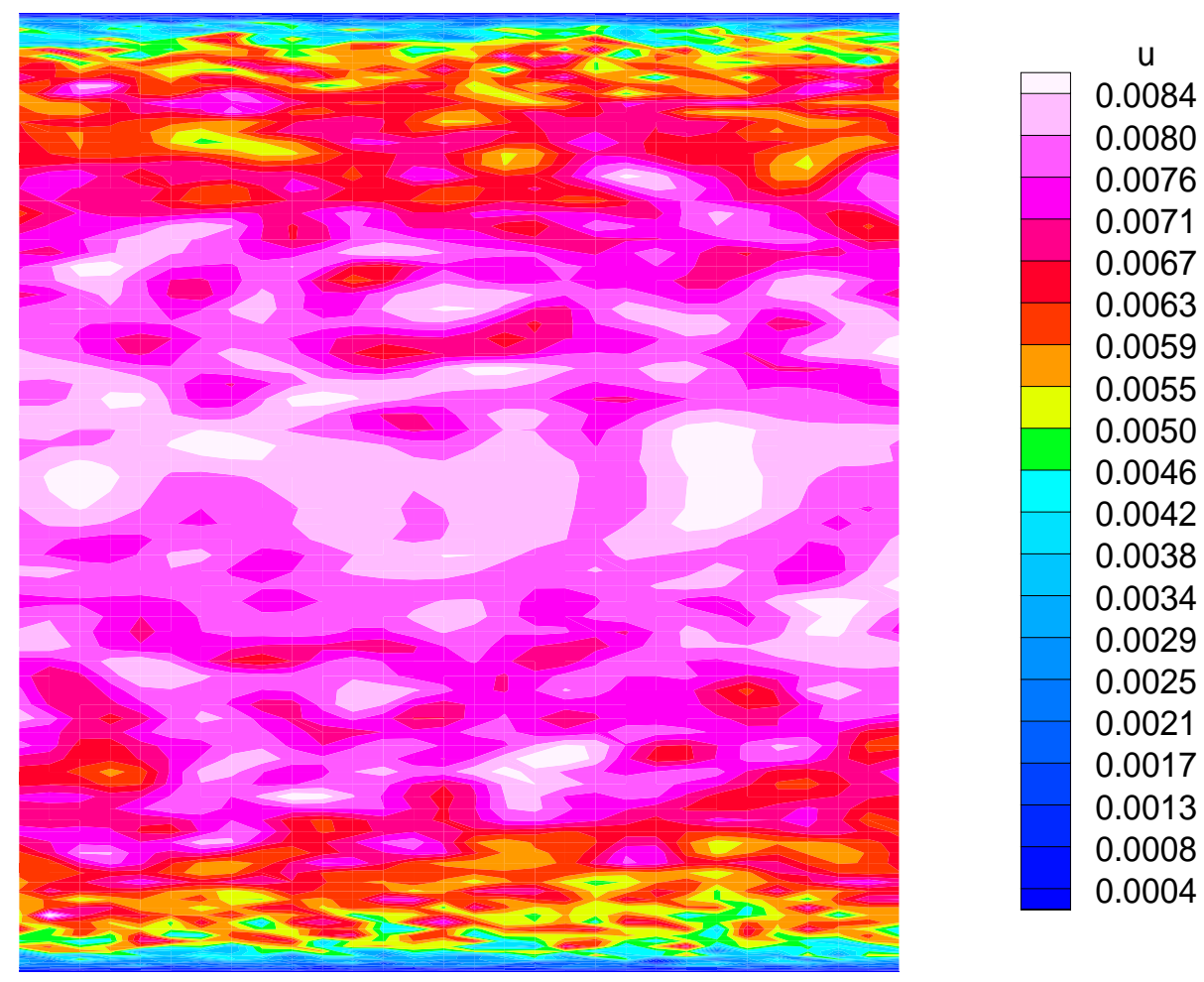

Figure 4.1: Contours of the u-velocity at $x-y$ plane 

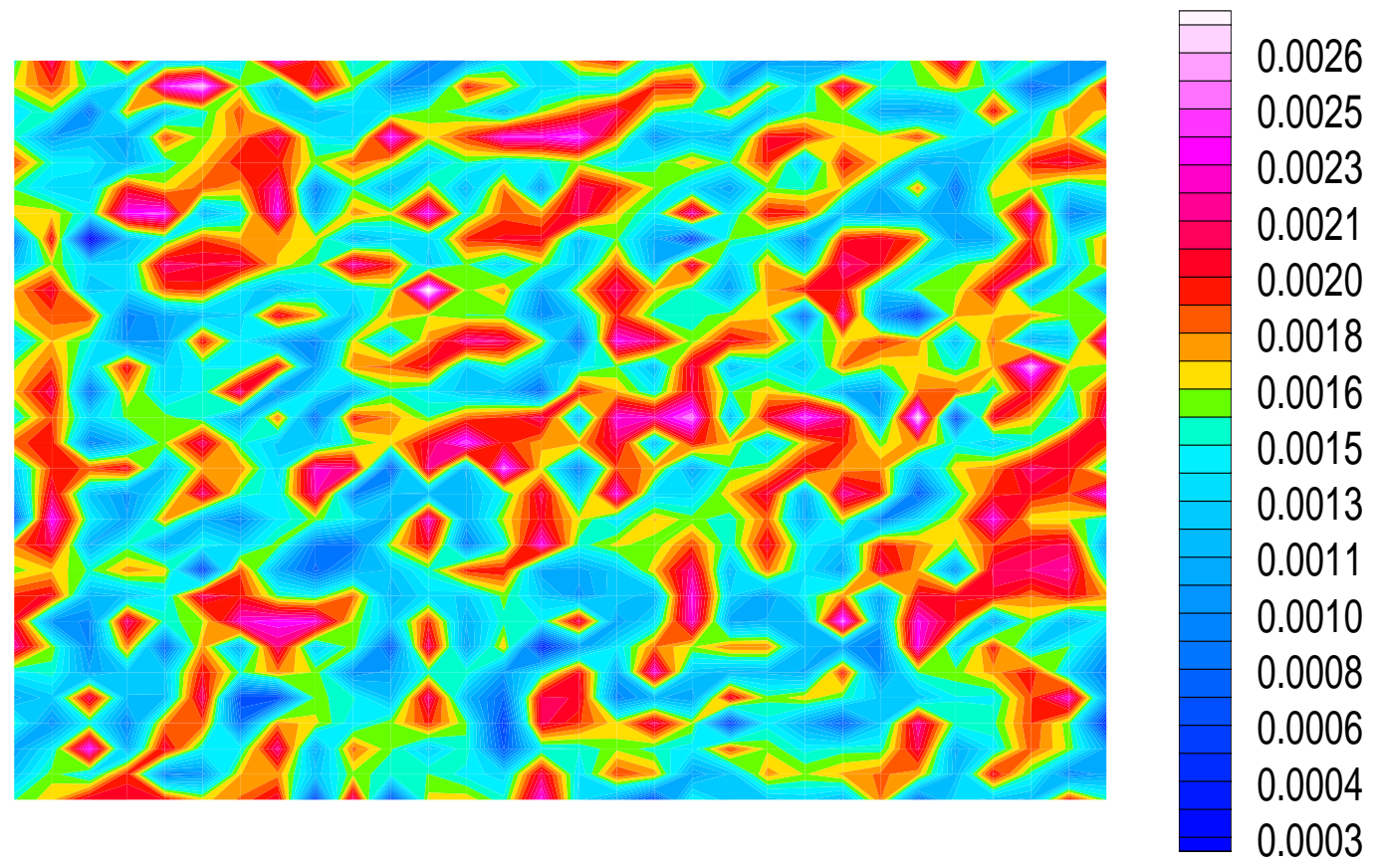

Figure 4.2: Contours of the u-velocity at $x-z$ plane parallel to the wall 


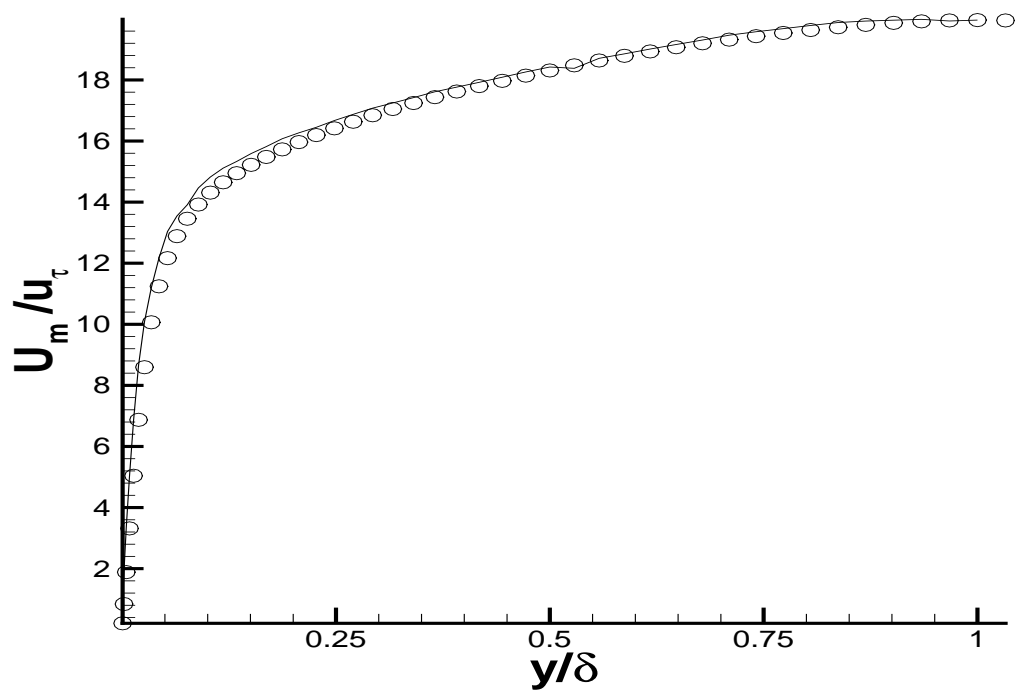

Figure 4.3: The mean velocity profile, o: DNS; _—: RFG

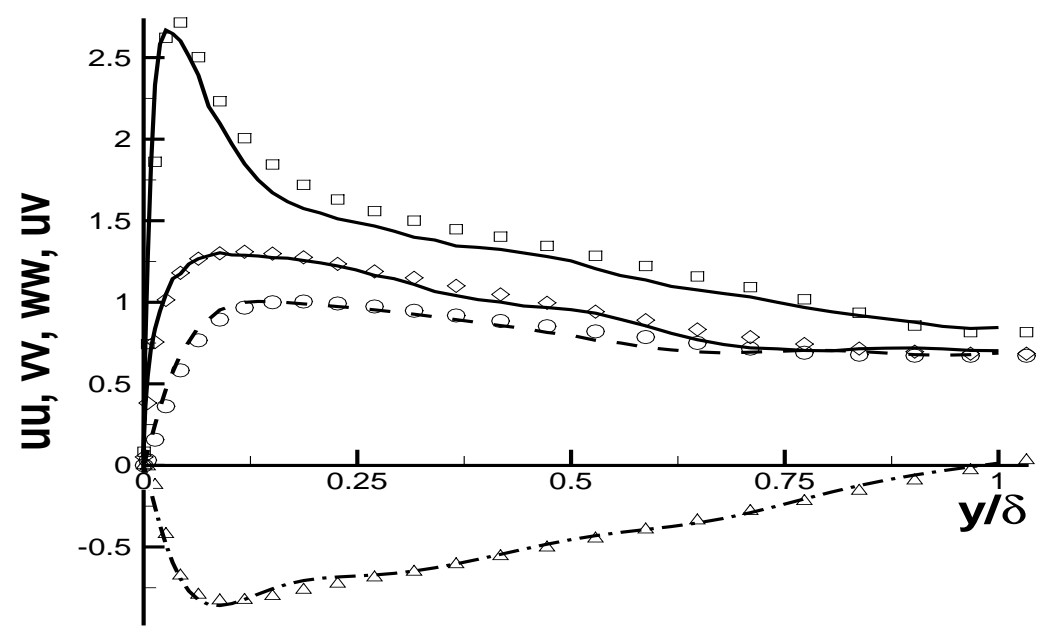

Figure 4.4: The Reynolds stresses. $\square: \overline{u u}$ of DNS, ○: $\overline{v v}$ of DNS, $\diamond: \overline{v v}$ of DNS, $\Delta: \overline{u v}$ of DNS; - : $\overline{u u}$ of RFG, $----: \overline{v v}$ of RFG, $\cdots \cdots: \overline{v v}$ of RFG, $-\cdot-\cdot-: \overline{u v}$ of RFG. 


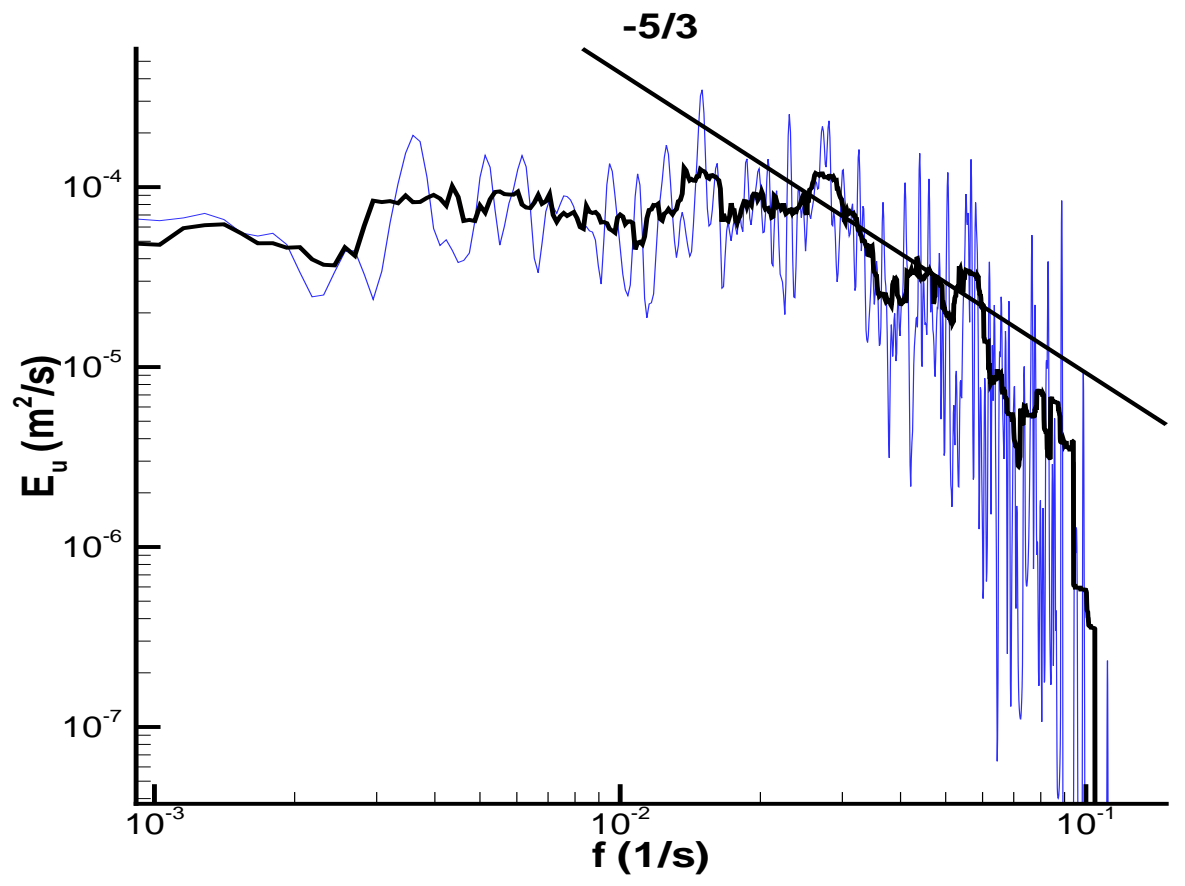

Figure 4.5: The energy spectra of axial velocity fluctuations at $y / \delta=0.02$ 


\section{Chapter 5}

\section{One Equation Subgrid-scale Model}

In Chapter 3, the numerical methods have been briefly described to compute incompressible flows. A summary of the Smagorinsky-based subgrid-scale models has been made. As have been discussed in Section 3.2, the development of a SGS model capable to represent the effect of the unresolved motion is essential in LES of turbulent flows. Since then one wishes to keep the computational domain as large as possible for the present ship wake simulation, there is a need to find a SGS model which would give good resolution even with relatively coarse grids. This is the subject of this chapter.

\subsection{Introduction}

There are many SGS models being used in LES today. Some of these are the Smagorinsky model(Smagorinsky, 1963), scale similarity model or mixing model(Bardina, 1989), dynamic model(Germano et al., 1991), dynamic mixed model (Zang et al., 1993), oneequation model (Sohankar et al., 2000), monotone integrated LES model (Boris et al., 1992), and those based on the estimation of unresolved scales of turbulence (Domaradzki 
and Saiki, 1997). Studies have been carried out to assess many of these models (Fureby et al., 1997; Vreman et al., 1997; Jordan, 2001). It is not easy to determine which SGS models generate the best results. One of the reasons is that the true behavior of a turbulent flow may well change depending upon the specific case. Another factor relevant to LES is the selection of the filter, which becomes important for complex geometry flows. Matters of this topic have been experimentally studied (Vasilyev et al., 1998). More recently, Magnient et al. (2001) summarized the theoretical studies on LES filters and kinetic energy spectra. They presented a mesh-independent filtering length larger than the mesh size and applied it together with the Smagorinsky model and the Schumann model (Schumann, 1975). To the author's knowledge, the Schumann model was the first one equation subgrid scale model developed for LES.

The idea of the one equation subgrid-scale model in LES comes from the one equation model (C.Wilcox, 1993) of RANS type. Normally, as in this study, one equation refers to the turbulent kinetic energy equation. An important feature of a one equation model is that no assumption is made of local balance between the subgrid scale energy production and dissipation rate. Therefore, it is expected that this model would be much better than the algebraic eddy viscosity model having constant coefficients in regions where local equilibrium is violated. Yoshizawa and Horiuti (1985) and Horiuti (1985) developed a one-equation model and applied it to channel flow. Comparison was made between this model and the Smagorinsky model, which is a special case of one-equation model when the equilibrium between production and dissipation of the SGS kinetic energy is assumed (Piomelli, 1997). The one-equation model gave better results, especially near the wall where the assumption of homogeneity is no longer valid.

Among the advantages of a one equation model is the fact that the independent definition of the velocity scale results in a more accurate prescription of the SGS time scale compared to algebraic eddy-viscosity models (Piomelli, 1999b). Lewellen (1977) pro- 
posed a one equation model and applied it to the atmospheric flow simulations. This model works very well in their LES of the atmospheric study and is able to capture the main eddies in the air flows. A rotational damping term was added to this model recently and it has been successfully applied to simulate tornado flows (Lewellen et al., 2000). Menon and Yeung (1995) proposed a one equation subgrid-scale model with or without a stochastic backscatter forcing term. These results showed that the subgrid stresses and the energy fluxes predicted by the subgrid-scale model correlated very well with the experimental data. Comparisons between this one equation model and a scale similarity model (Liu et al., 1994a) showed that when the grid-cell size decreases, the one-equation model gave better predictions (higher correlation). The results also showed that to use the scale similarity assumption relatively fine grid resolution was required, whereas, the kinetic energy model could be used even on relatively coarse grids. Later this one equation model has been improved by Menon et al., (1996) and was named as a dynamic one-equation model. The main objective of the dynamic one-equation model was to improve the representation of the dissipation term, which was poorly resolved in the previous model. Thus, in the dynamic one-equation model, the constants for modeling the diffusion and the dissipation were determined dynamically, analogous to those in other dynamic models (Germano et al., 1991), based on the assumption of the similarity of the stresses between the subgrid scale and the test scale. Recently, different versions of the dynamic one-equation model have been developed (Sohankar et al., 2000; Ghosal et al., 1995). The least squares approach is applied to reduce the number of constants within the filtering operation. Some constrained variational formulations were used to avoid the instability. However, all of the methods, including the local least-squares method and the volume-averaging prescription method, were designed to prevent instability in the calculation by ruling out backscatter in one way or the other.

On the other hand, the inclusion of a backscatter model is desirable because this phe- 
nomenon has been shown to be important. By using DNS results, Piomelli et al. (1991) indicated that about $50 \%$ of the grid points were experiencing backscatter. Moreover, the backscatter and forward scatter contributions to the SGS dissipation were comparable, and each is often larger than the total SGS dissipation. The backscatter increases with Reynolds number while the magnitude of the SGS dissipation increases with filter width. The conclusion is: for nonequilibrium flows, backscatter must be taken into account to ensure accurate prediction by LES. A comprehensive review about the backscatter energy has been done by Domaradzki et al.(1997).

The Smagorinsky model is a purely dissipation model and can not account for the backscattering energy. Other Smagorinsky-type models, including the mixture model, dynamic model, and mixed dynamic model, may involve the backscatter energy, but they do not explicitly contain any information regarding the total amount of energy contained in the subgrid scales. The happening of the backscatter totally depends on the assumption of the similarity of the shear stresses between the test scale and grid scale. Therefore, if the proportionality coefficient in the eddy viscosity relation becomes negative in any part of the domain, then the backscatter happens which means that part of the energy is transferred back from the subgrid-scale to the resolved scale. However, the model itself do not have any information on the available energy in the subgrid scales and is therefore unable to provide the mechanism to saturate the reverse flow of energy. Moreover, in a physical system, if all the energy of the subgrid scales is removed the subgrid-scale stress should go to zero, thus quenching the reverse flow of energy. Clearly, a more elaborate model that keeps track of the subgrid-scale kinetic energy $k$ is required. But the subgrid-scale kinetic energy transport equation itself does not describe the reverse flow of energy from the subgrid to resolved scales, i.e. backscatter, so other terms responsible for energy reversal might be required. The energy transfer to/from the subgrid-scales can be divided in two types. One is the effect of the very small eddies representing the dissipation which can be easily modeled 
by applying a positive eddy viscosity. The other is the effect of the intermediate size eddies which can be thought to be responsible for the phenomenon of backscatter. There are some methods designed to model the effect of the intermediate-size eddies. Among them are:

1. Gradient diffusion model (Clark et al., 1979);

2. An addition of a stochastic force to the eddy viscosity term (Chasnov, 1991; Mason and Thomson, 1992; Leith, 1990).

Although some fairly good results have been reported through those methods, theoretically the models are not quite satisfactory. Modeling backscatter using a stochastic noise totally uncorrelated from one time-step to the next implies that the correlation time of the intermediate eddies is much shorter than that of the smallest resolved eddies. Obviously this is not the case since both of them should not be so different, at least being of the same order of magnitude. Moreover, the backscatter also has the feature of uncertainty (Piomelli et al., 1991) which is strongly dependent on the filter type. These issues have to be better understood before any meaningful assessment of the importance of backscatter in LES and a better way to represent it can be achieved.

In the following sections, first the mathematical description of the subgrid-scale kinetic energy transport will be derived. Then a one equation model, in which the backscatter energy is included, will be proposed. The validation will be performed next followed by the summary of the results.

\subsection{Subgrid-scale Kinetic Energy Equation}

The subgrid kinetic energy $k_{s g s}$ is defined as,

$$
k_{s g s}=\frac{1}{2}\left(\overline{u_{i} u_{i}}-\bar{u}_{i} \bar{u}_{i}\right)
$$


The equation for $k_{s g s}$ is first derived by filtering the equation of the kinetic energy $u_{i}^{2} / 2$ and subtracting from it the exact equation for the resolved kinetic energy $\bar{u}_{i}{ }^{2}$. After modeling the dissipation, diffusion, and pressure term, the resulting equation can be written as

$$
\frac{\partial k_{s g s}}{\partial t}+\frac{\partial}{\partial x_{j}}\left(\bar{u}_{j} k_{s g s}\right)=\tau_{i j} \frac{\partial \bar{u}_{j}}{\partial x_{j}}-C_{D} \frac{k_{s g s}^{3 / 2}}{\Delta}+\frac{\partial}{\partial x_{j}}\left[\left(\nu_{T}+\nu\right) \frac{\partial k_{s g s}}{\partial x_{j}}\right],
$$

where the Reynolds stress tensor $\tau_{i j}$ is given by:

$$
\tau_{i j}=\overline{u_{i} u_{j}}-\bar{u}_{i} \bar{u}_{j}
$$

and the strain rate tensor is defined as:

$$
\bar{S}_{i j}=\frac{1}{2}\left(\frac{\partial \bar{u}_{i}}{\partial x_{j}}+\frac{\partial \bar{u}_{j}}{\partial x_{i}}\right)
$$

The essential idea of one-equation SGS model is that the eddy viscosity is a function of the SGS turbulent kinetic energy while an eddy-viscosity assumption is used in modeling the Reynolds stresses, i.e.

$$
\tau_{i j}=-2 \nu_{T} \bar{S}_{i j}-\frac{2}{3} k_{s g s} \delta_{i j}
$$

and

$$
\nu_{T}=C_{k} k_{s g s}^{1 / 2} \Delta
$$

where $\Delta$ is the length scale which is defined as $\Delta=\left(\Delta x_{1} \Delta x_{2} \Delta x_{3}\right)^{1 / 3}$. The model constants are chosen as, $C_{k}=0.09, C_{D}=0.9$ and $\sigma_{k}=1.0$ (Menon and Yeung, 1995).

If unresolved scales have energy-containing eddies, then there is a good chance for backscatter of energy from the subgrid scales to the resolved scales. An earlier study (Chasnov, 1991) has shown that forward scatter (by eddy viscosity term) and backscatter are two distinct processes. Therefore, these two effects must be modeled separately. Menon and Yeung (1995) assume that the backscatter effect can be modeled by a random force, which satisfies certain constraints. This model can be written as follows 


$$
\tau_{i j}^{b s}=C_{b s} \prod \frac{\Delta^{2}}{\sqrt{\Delta t}}|\bar{S}| \bar{S}_{i j}^{1 / 2} .
$$

Here $C_{b s}$ is a constant of order unity. $\Delta t$ is the time step of the LES. $\prod$ is a random number with zero mean and unit variance. Then the modeled SGS stresses $\tau_{i j}$ become

$$
\tau_{i j}=-2 \nu_{T} \bar{S}_{i j}-\frac{2}{3} k_{s g s} \delta_{i j}+\tau_{i j}^{b s}
$$

As discussed in the last section, modeling backscatter as a stochastic noise totally uncorrelated from one time-step to the next leads to some unphysical features. Another fact is that the influence of the filter should be included according to the investigation by Piomelli et al. (1991). To overcome these deficiencies, here a SGS model is proposed in which the backscatter energy is included by applying the RFG approach (Chapter 4). The resulting form of the backscatter contribution to the subgrid stress model can be written as:

$$
\tau_{i j}^{b s}=f\left(k_{s g s}, l, \tau\right)
$$

here $l$ and $\tau$ are turbulence length scale and time scales, respectively, and $f$ represents the RFG algorithm.

The subgrid scale kinetic energy is applied as the input of RFG. There are two benefits by doing this. First, it will guarantee that there is energy available to transfer back, and the energy transferred between the GS (Grid Scale) and SGS is limited by the amplitude of the subgrid scale energy. Secondly, by using the parameters of time scale and length scale, the energy correlation between time steps and filter influence can be resolved. It should be noted that the RFG function $\mathrm{f}$ is not a totally random function. The SGS model now can be expressed as

$$
\tau_{i j}=-2 C_{k} k_{s g s}^{1 / 2} \Delta \bar{S}_{i j}-\frac{2}{3} k_{s g s} \delta_{i j}+\tau_{i j}^{b s}
$$


Substitution of Eqn. 5.9 and Eqn. 5.10 into Eqn. 5.2 gives

$$
\frac{\partial k_{s g s}}{\partial t}+\frac{\partial}{\partial x_{j}}\left(\bar{u}_{j} k_{s g s}\right)=\frac{\partial}{\partial x_{j}}\left(C_{k} k_{s g s}^{1 / 2} \Delta \frac{\partial k_{s g s}}{\partial x_{j}}\right)+\tau_{i j} \frac{\partial \bar{u}_{j}}{\partial x_{j}}-C_{D} \frac{k_{s g s}^{3 / 2}}{\Delta}
$$

$C_{k}$ and $C_{D}$ are calculated by using the same strategy as the dynamic eddy viscosity model, dynamic one equation subgrid scale model (Sohankar et al., 2000; Kim et al., 1999). This approach is described briefly following Sohankar et al.(2000). The dynamic coefficient $C_{k}$ in the production term is computed as

$$
\begin{gathered}
C_{k}=\frac{L_{i j} M_{i j}}{2 M_{i j} M_{i j}}, \\
L_{i j}={\widehat{u_{i} \bar{u}_{j}}}-\widehat{u_{i}} \widehat{\bar{u}}_{j}, \\
M_{i j}=\widehat{\Delta} \widehat{K^{1 / 2}} \widehat{\bar{S}_{i j}}-\Delta k_{s g s}^{1 / 2} \bar{S}_{i j}, \\
K=k_{s g s}+\frac{1}{2} L_{i i} .
\end{gathered}
$$

Here $L_{i j}$ denotes the dynamic Leonard stresses, and $K$ is the subgrid kinetic energy on the test level. The test filter is twice as large as the grid filter, i.e., $\widehat{\Delta}=2 \Delta$.

To estimate $C_{D}$ is not as straightforwards as $C_{k}$. Using $C$ for the energy convective term, $D$ for the diffusion term, and $P$ for the production term, i.e.

$$
\begin{aligned}
C_{k_{s g s}} & =\frac{\partial k_{s g s}}{\partial t}+\frac{\partial}{\partial x_{j}}\left(\bar{u}_{j} k_{s g s}\right), \\
D_{k_{s g s}} & =\frac{\partial}{\partial x_{j}}\left(C_{k} k_{s g s}^{1 / 2} \Delta \frac{\partial k_{s g s}}{\partial x_{j}}\right),
\end{aligned}
$$

and 


$$
P_{k_{s g s}}=\tau_{i j} \frac{\partial \bar{u}_{j}}{\partial x_{j}},
$$

the subgrid scale $k$ equation can be written as:

$$
C_{k_{s g s}}-D_{k_{s g s}}=P_{k_{s g s}}-C_{D} \frac{k_{s g s}^{3 / 2}}{\Delta} .
$$

A similar formulation can be written for $K$,

$$
C_{K}-D_{K}=P_{K}-C_{D} \frac{K^{3 / 2}}{\widehat{\Delta}} .
$$

Assuming that similarity exists between the test filter and grid filter, after filtering Eqn. 5.19 and combining it with Eqn. $5.20 C_{D}$ can be obtained as:

$$
C_{D}^{n+1}=\left(P_{K}-\widehat{P_{k_{s g s}}}+\frac{1}{\Delta} \widehat{C_{D}^{n} \hat{k}_{s g s}^{3 / 2}}\right) \frac{\widehat{\Delta}}{K^{1 / 2} \widehat{k_{s g s}}}
$$

The dissipation cannot be negative, which requires $C_{D}$ to attain nonnegative values. In Eqn. 5.21 $C_{D}$ is kept inside the filtering process and is taken from the previous time step.

\subsection{Solution to the SGS Turbulent Kinetic Energy Equa-}

\section{tion}

The SGS $k$-equation is similar to the generalized scalar equation described in Chapter 2 except that there are two more terms, the production term and the dissipation term. However, if the equation is solved explicitly, then these two extra terms can be treated as source terms. Thus the SGS $k$ equation can be solved by using the same methods as those for the scalar equation. 


\subsection{Near Wall Modification}

In the wall region it is always very difficult to resolve the sublayer especially for high Reynolds number flow, even when the dynamic one-equation model is applied. Because of the numerical instabilities, the dynamic coefficients have to be averaged through the flow field. This process eliminates the advantage of the dynamic model which is supposed to resolve the near wall problems.

There are many different ways to account for wall effects. Wilcox (1993) has done very comprehensive reviews about modeling wall bounded flows by using so called LowReynolds-Number model. Hwang and Lin (1998) proposed a model by using the Taylor microscale in the damping function and including the pressure diffusion terms in the equation. Goldberg et al.(1990) use a hybrid $k-L$ model to account for the near wall influences by altering the formulas for $k$ and $L$ in the near wall region, while Lewellen (1977) added a new term in the $k$ equation. As for LES, Cabot and Moin (2000) reviewed the popular methods in dealing with the near wall resolution problem and discussed the achievements and discrepancies of the near wall treatments up to date.

In this study, Goldberg's method was adopted as a base model and some modifications have been made.

The dissipation rate of the energy is modeled in terms of $k$ and a length scale which should guarantee proper behavior from the wall to the fully turbulent region, that is

$$
\epsilon=2 A_{\epsilon} k_{\text {sgs }}^{3 / 2} /\left[y\left(1-e^{-A_{\epsilon} R_{y}}\right)\right]
$$

where $A_{\epsilon}=C_{\mu}^{3 / 4} / 2 \kappa, \kappa=0.4, C_{\mu}=0.09, R_{y}=\sqrt{k_{s g s}} y / \nu$, and $y$ is the distance from the wall.

From Eq. 5.22 it can be seen that at the wall the dissipation becomes zero because $k$ tends to zero there. This is not physical because at the wall the dissipation is balanced by 
the molecular diffusion which has a limited value. Thus the dissipation term is modified as:

$$
\epsilon=2 A_{\epsilon} k_{s g s}^{3 / 2} /\left[y\left(1-e^{-A_{\epsilon} R_{y}}\right)\right]+2 \nu \frac{k_{s g s}}{y^{2}}
$$

The velocity scale, needed to complete the formulation for the eddy viscosity field, is given by

$$
u=\sqrt{k_{s g s}}
$$

The entire eddy viscosity field is given by

$$
\nu_{T}=C_{k} f_{\mu} k_{s g s}^{1 / 2} \Delta
$$

$f_{\mu}$ is a near-wall function and is given by (Goldberg, 1991)

$$
f_{\mu}=1+\left[A_{\epsilon} \sqrt{k^{+}} y^{+}\left(1-e^{-A_{\epsilon} \sqrt{k^{+}} y^{+}}\right)\right]^{-1 / 2}\left(1-e^{-A_{\mu} \sqrt{k^{+}} y^{+}}\right)
$$

where $y^{+}=y u_{\tau} / \nu, k^{+}=k_{s g s} / u_{\tau}^{2}, u_{\tau}=\sqrt{\tau_{w} / \rho_{w}}$, and $A_{\mu}=0.016$.

\subsection{Calculation of the Backscatter Energy}

The backscatter energy is included in the present one-equation SGS model by applying the RFG method. The main idea is to import a reasonable amount of energy from the unresolved small structures into the large scale structures. In some sense, this strategy is similar to those used in other studies(Menon et al., 1996; Leith, 1990). However, in these studies the backscatter energy is mainly based on the approximation that the energy is proportional to the strain rate tensor using scale analysis. The modeled terms are multiplied by a random number and added to the momentum equation as a random force. Such a scheme is more 
or less arbitrary because the more important physical parameters, such as the subgrid time scale or the subgrid scale energy, are not included. To overcome these inadequacies, in the present model, two important turbulence parameters, namely, the subgrid length scale and time scale, are included in the stochastic term based on the real subgrid turbulent kinetic energy itself.

Two approaches have been proposed to implement the backscattering process.

\subsubsection{Superimposition of the velocity fluctuations on the resolved flow field —Explicit Superimposition Method (ESM)}

Assuming a specific percentage of subgrid scale turbulent kinetic energy $(k)$ can be involved in the inverse interaction between the grid scale and subgrid scale one can use it as the input for RFG. The volume size is selected as the length scale for RFG because the energy transfer mainly takes place between the smallest resolved structures and the biggest unresolved structures. The velocity scale is calculated from the square root of $k$. Time scale can be calculated from length scale and velocity scale.

The condition for the backscattering is realized whenever the time interval (integral time from last backscattering) is bigger than a specified time, for example, the Taylor time scale or the Kolmogorov time scale.

The Taylor time scale is computed (Tennekes and Lumley, 1997) from

$$
\tau_{T}=\lambda / u=(15 \nu l)^{1 / 2} / u^{3 / 2}
$$

where $\lambda$ is the the Taylor length scale. In Eq. 5.27, $l$ and $u$ can use the length scale and time scale of RFG.

The Kolmogorov time scale can be computed from

$$
\tau=(\nu / \epsilon)^{1 / 2}
$$


Here $\nu$ is kinematic viscosity, and $\epsilon=k^{3 / 2} / l$ is dissipation rate.

The wall damping was also applied for backscatter energy by reducing the ratio of the backscatter energy to the total SGS turbulent kinetic energy.

After RFG a sub-turbulent random flow field with smaller magnitude of turbulence intensity was generated. As it was discussed in Chapter 4, this sub-flow field, which could be called subgrid fluctuations, satisfied continuity. Thus the whole flow field still obeys mass conservation after the addition of the subgrid fluctuations. Besides, some iterations will be performed before another "backscattering" happens. Therefore the overall flow field essentially satisfies the Navier-Stokes equations.

\subsubsection{Superimposition of backscatter stresses in both $k$-equation and}

\section{momentum equation — Implicit Superimposition Method (ISM)}

Instead of adding the subgrid fluctuations directly on the resolved flow field, they are used to create a sort of stress, namely subgrid stresses, and then these subgrid stresses are added to the Reynolds stresses in both the momentum equation and the subgrid scale $k$ equation. The way to compute the subgrid stresses is by averaging the product of the subgrid fluctuations $\overline{v_{i} v_{j}}$ in space, i.e.

$$
\tau_{i j}^{b s}=\overline{v_{i} v_{j}}
$$

Here $v$ is the subgrid fluctuations.

Superimposing the subgrid stresses on the Reynolds stresses is quite straightforward in the $k$ equation. However, in the momentum equation it is more complicated and is explained below.

The Reynolds stresses without backscattering in the momentum equation is split into two parts. One is combined with the molecular diffusion term, another is treated as a source 
term (see Eq. 2.33 and Eq. 2.34). To make the backscattering approach simple, the subgrid stresses are added as source terms in the momentum equation. Similar to Eq. 2.34, these source terms are rewritten as:

$$
\bar{S}_{i}=\frac{\bar{\rho}^{\prime}}{\rho_{0}} g \delta_{i} 2+\frac{\partial \nu_{T}}{\partial x_{j}} \frac{\partial \bar{u}_{j}}{\partial x_{i}}-C_{1} \frac{\partial L_{i j}^{m}}{\partial x_{j}}+\frac{\partial \overline{v_{i} v_{j}}}{\partial x_{j}} .
$$

After coordinate transformation the source term (Eq. 2.53) appears like:

$$
\bar{S}_{i}=J^{-1} \frac{\partial \xi_{m}}{\partial x_{j}} \frac{\partial \xi_{n}}{\partial x_{i}} \frac{\partial \nu_{T}}{\partial \xi_{m}} \frac{\partial \bar{u}_{j}}{\partial \xi_{n}}-C_{1} \frac{\partial}{\partial \xi_{m}}\left(J^{-1} \frac{\partial \xi_{m}}{\partial x_{j}} L_{i j}^{m}\right)+\frac{\partial}{\partial \xi_{m}}\left(J^{-1} \frac{\partial \xi_{m}}{\partial x_{j}} \overline{v_{i} v_{j}}\right)
$$

Note, the density fluctuations have been ignored.

As in approach 1, the wall damping was also applied for backscatter energy.

\subsection{Application to the Channel Flow}

The channel flow is always a good choice to test a newly developed numerical scheme or turbulence model. Although the geometry is simple, it contains most of the flow features in turbulent flows. The Reynolds number based on half of the channel height and the mean center velocity was 33000 , which is the same as it was in Chapter 3. Three different grids have been used to test the one equation model. The parameters and grid resolution used are given in Table 5.1

The grid is uniform in both streamwise $(x)$ and spanwise $(z)$ directions, but nonuniform in the vertical $(y)$ direction, with fine grids near the wall. Non-slip boundary conditions are used on the wall, while periodic boundary conditions are applied in the other directions. The initial condition is that the velocity field is at rest with no random perturbations. All the statistics were taken after the flow was fully developed, with at least 20 characteristic flow times (particles past the flow domain 20 times). The instantaneous velocities on 


\begin{tabular}{|c|c|c|c|c|c|}
\hline Case & SGS Model & Grid points & Domain size (/delta) & $C_{S}$ or $C_{K}$ & $C_{D}$ \\
\hline I & Smagorinsky & $(34,34,34)$ & $4 \pi \times 1 \times 2 / 3 \pi$ & 0.065 & - \\
\hline \hline II & OEM & $(34,34,34)$ & $4 \pi \times 1 \times 2 / 3 \pi$ & 0.05 & 0.10 \\
\hline \hline III & OEM & $(50,50,50)$ & $4 \pi \times 1 \times 2 / 3 \pi$ & 0.05 & 0.10 \\
\hline \hline IV & OEM & $(66,66,66)$ & $4 \pi \times 1 \times 2 / 3 \pi$ & 0.05 & 0.10 \\
\hline
\end{tabular}

Table 5.1: Parameters for large eddy simulation of channel flow using One-equation model three different vertical lines were saved for turbulence statistics. Then all the mean flow characteristics, such as mean velocity and turbulence intensities on these three lines were averaged and projected on half of the channel height. The final mean velocities were taken by weighting the space averaging and time averaging.

\subsubsection{Results of one equation model}

To test the one equation ( $K$ equation) model, three cases with different grid numbers have been computed (Table 5.1). Simulations with the Smagorinsky model have also been performed in Case $I$ using the same grid as in Case $I I$ in order to verify the one equation model.

The mean streamwise velocity along the vertical line for Case $I I I$ and Case $I V$ is presented in Fig. 5.1. It has not been converted into $y^{+}-u^{+}$because not enough data have been accumulated yet. However from this figure it is seen that the logarithm range is captured. In Case $I$ and Case $I I$, this range has not been resolved because of relatively coarse grid being used. This issue will be discussed later.

The mean subgrid scale turbulent kinetic energy $\left(k_{s g s}\right)$ along the vertical line is shown in Fig. 5.2 for Case $I I$ and Case $I V$. Case $I I I$ is in between and has been omitted. $k_{\text {sgs }}$ is decreasing with the increasing of the grid number. This is the right trend because for 
finer grids more energy-bearing eddies will be resolved in LES thus leaving less energy to transfer to the subgrid scales. When the grids are fine enough to resolve the smallest eddies (Kolmogorov scale), then LES becomes DNS and $k_{s g s}$ will tend to be zero. Correspondingly, the turbulence viscosity should also drop down when fine grids are used. This feature is confirmed in Fig. 5.3.

The comparison of the turbulence intensities between Case $I$ and Case $I I$ is presented in Fig. 5.4 to Fig. 5.6. Because the logarithm range has not been resolved in these two cases, the friction velocity $u_{\tau}$ is calculated by using the empirical relationship (Hussain and Reynolds, 1975). In both cases the same $u_{\tau}$ was used in order to compare the results. It can be seen that $u_{r m s}$ from the one equation model is larger than those from the Smagorinsky model and measurements. But the position of the maximum $u_{r m s}$ from the one equation model is much closer to that of the measurements. The other two components, $v_{r m s}$ and $w_{r m s}$, are almost the same. Therefore, if the total turbulent kinetic energy is considered, then it appears that the one equation model can capture more turbulence structures. The energy spectra, Fig. 5.7, clearly show that the resolved energy of the one equation model, the area under the curve, is higher than that of the Smagorinsky model.

To validate the one equation subgrid-scale model and the solver for the subgrid scale turbulent kinetic energy (TKE), the resolved TKE and the subgrid-scale TKE for case $I V$ are shown in Fig. 5.8. The TKE from the measurements is also depicted in this figure. Note that $k$ has been nondimensionized by $u_{\tau}^{2}$. The overall subgrid-scale TKE is under twenty percent which means the grid is fine enough to capture most of the turbulence. However, near the wall, it seems that the grid is not fine enough to resolve the boundary layer. The peak point is too close to the wall. The subgrid-scale TKE estimated from the Smagorinsky model is also presented in Fig. 5.8. It is comparable to the subgrid-scale TKE resulted from the subgrid-scale TKE equation. This implies that the solver for the subgrid-scale TKE is appropriate. 
Dynamic one equation model, where the constants $C_{k}$ and $C_{D}$ are computed locally, has been implemented in the computer code. But to achieve numerical stability, these constants have to be smoothed along the whole domain, thus giving no benefit. The results will not be presented here and focus will be put on the one equation model with energy backscatter.

\subsubsection{Backscatter results and discussion: approach 1}

In this approach, the backscatter is implemented by superimposing the velocity fluctuations on the resolved flow field directly. This procedure will be active whenever the criteria cited above are reached. Adding random fluctuations onto the resolved velocity may not sound physical. However it really depends on what kind of fluctuations were added. In this study, the fluctuations are obtained by reproducing the small scale turbulence (subgrid scale turbulence) field which can not be resolved. This small scale field is based on the subgrid scale turbulent kinetic energy $\left(k_{s g s}\right)$, subgrid length scale and subgrid time scale which is computed from $k_{s g s}$ and subgrid length scale. As have been verified by Smirnov et al. (2001b), the fluctuations thus generated satisfied continuity. Therefore, adding such a small scale turbulence flow field will not greatly alter the physical behavior of the main flow field, rather it feeds back some reasonable energy (carried by those small eddies) through which the flow field will be rearranged and directed to the more physical outcomes.

The mean velocity is shown in Fig. 5.9 compared with that of the case without backscatter. There are slight differences and the logarithm range is still not resolved well.

The turbulence intensities have been changed significantly by including the backscatter. The streamwise component, Fig. 5.10, is somewhat reduced and comes closer to the measurements. The mechanism for this result is not very clear but it can be explained to some extent. From the last section it can be found that the turbulence intensities are strongly anisotropic. The streamwise component is unphysically larger than the other two 
components, which implies that somehow the fluid particles are much easier to oscillate in the streamwise direction. After including the small scale fluctuations, which are isotropic those disparities could be averaged to some degree. Thus the energy of flow field will be redistributed. This analysis is also confirmed by the noticeable change of the turbulence intensities in the other directions, Fig. 5.11 and Fig. 5.12. Both have increased.

The instantaneous subgrid scale turbulent kinetic energy on the center vertical plane is presented in Fig. 5.13 to compare with that without backscatter which is shown in Fig. 5.14. Before backscatter is included, the subgrid scale turbulent kinetic energy is smoothly distributed on the plane. With backscatter, subgrid scale turbulent kinetic energy is clearly effected and redistributed among the turbulence structures.

To show the detail flow field, comparisons of the velocity contours, with the same contour levels for both with backscatter and without backscatter, are depicted in Fig. 5.15 to Fig. 5.20. More turbulence structures, especially small eddies, are presented when backscatter is included.

However, despite some encouraging results have been obtained, many uncertainties still exist. The first one is how much out of the subgrid scale turbulent kinetic energy can serve as backscatter resource. Another one that comes into mind is how often the backscatter should happen. Needless to say that considerable work is still needed to resolve these issues.

\subsubsection{Backscatter results and discussion: approach 2}

The backscatter stresses have been added to the momentum equations, which can become a source when it is positive and a sink when negative. When it acts as a source term, backscatter is realized. Otherwise it provides another dissipation source to the modeled Reynolds stresses $\tau_{i j}$. Thus the constant $C_{k}$ in eddy viscosity formulation $\nu_{t}=C_{K} K^{1 / 2} \Delta$ 
needs to be adjusted. The empirical constant has been well tested and it is verified that it properly accounts for the global SGS dissipation. So to keep the total dissipation rate fixed, the constant $C_{K}$ has to be reduced. This conclusion is somehow different from those by Mason and Thomson (1992), in which the Smagorinsky constant is not changed, or needs to be slightly increased.

One circumstance that is worth mentioning is that with the backscatter included, the logarithmic velocity profile can be clearly reproduced (Fig. 5.21). This feature has also been investigated by Mason and Thomson (1992) for boundary flow simulations. When backscatter was modeled, the logarithm range was captured; otherwise the gradient near the wall was unrealistical.

The turbulence intensities computed with approach 2 have the same trend as in approach 1 but slightly better; see Fig. 5.22 to Fig. 5.24. The $r m s$-streamwise velocity components are still too large compared to the measurements. The reason may lie partially in the smaller empirical constant being used. However, as pointed out by Mason and Thomson (1992), and also was supported by numerical experiments performed by the present author, the improvements found with backscatter are not just a consequence of effectively constant adjusting or the length scale modification.

The subgrid scale turbulent kinetic energy presented in Fig. 5.25 also shows the influence of the backscatter, i.e. some turbulence structures appears compared to Fig. 5.14.

For completeness, the velocity contours at the center vertical plane and a horizontal plane close to the wall are depicted in Fig. 5.26 to Fig. 5.28. Clearly, more turbulence structures are present in the cases with backscatter compared to those without backscatter.

Based on the results in this section and last section, we may conclude that including energy backscatter in LES is feasible, and that better results can be expected. Both approaches proposed in this study provide a simple but physical and effective way to account for the energy backscatter, which is an observed feature in turbulent flows. The second approach, 
in which the backscatter stresses are superimposed on the Reynolds stresses in both the $k$ equation and the momentum equation is preferred since the process has a sounder physical basis. Nevertheless, more validation study is necessary.

\subsection{Summary}

In this Chapter subgrid scale models have been reviewed. The focus was mainly on the one equation model. A subgrid scale one equation model has been briefly derived. The dynamic modeling procedure has also been presented. Near wall modifications have been discussed and a combination of new approaches has been proposed. Results show that the proposed modifications are appropriate in LES. The new one equation model has been tested on channel flow by using three different grid sizes. The Smagorinsky model was also used for comparison.

Backscatter, which means that energy is transfered back from the subgrid scales to the resolved scales is an important factor in LES. In this study, two different approaches have been developed to model the backscatter: (i) Superimposing the velocity fluctuations on the resolved flow field; (ii) Superimposing backscatter stresses on the Reynolds stresses in both $k$-equation and momentum equation. It is well known that the sub-grid stress tensor correlates very poorly with the strain rate tensor (Pomraning and Rutland, 2001; Jimenez and Moser, 2000). By adding the backscatter part which is not correlated with the strain rate tensor the correlation can be reduced. Consequently, the resulted stresses may be more physical. The results obtained from the presently proposed approaches are encouraging. 


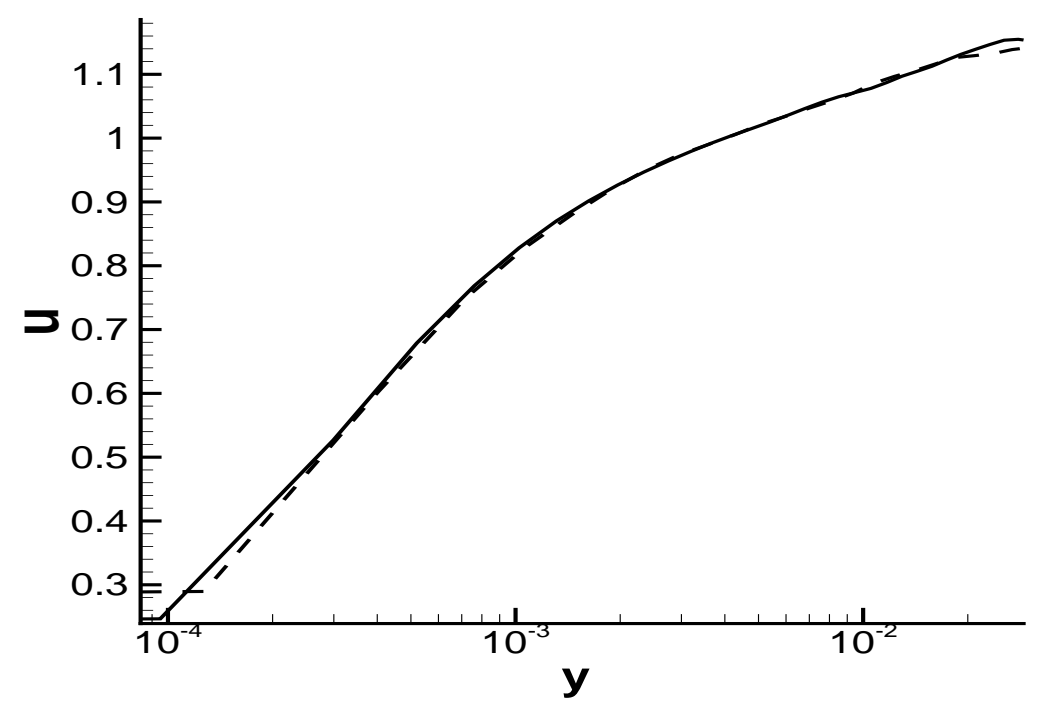

Figure 5.1: The mean velocity. - , case $I V ;----$, case $I I I$.

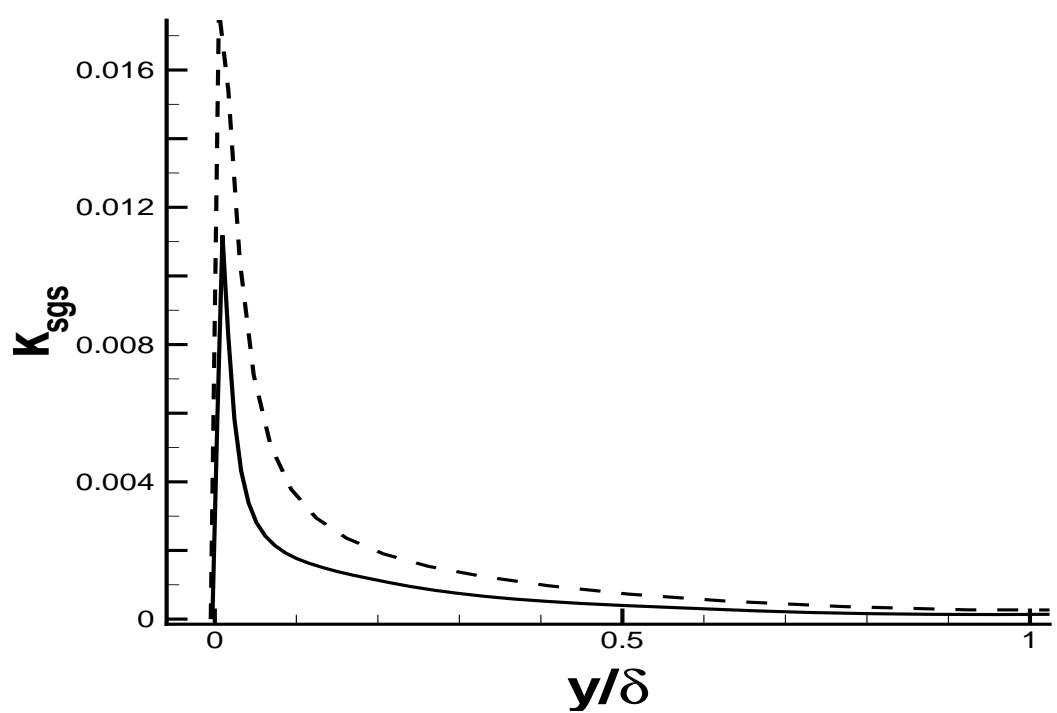

Figure 5.2: The mean subgrid scale turbulent kinetic energy. - , case $I V$; ---- , case $I I$. 


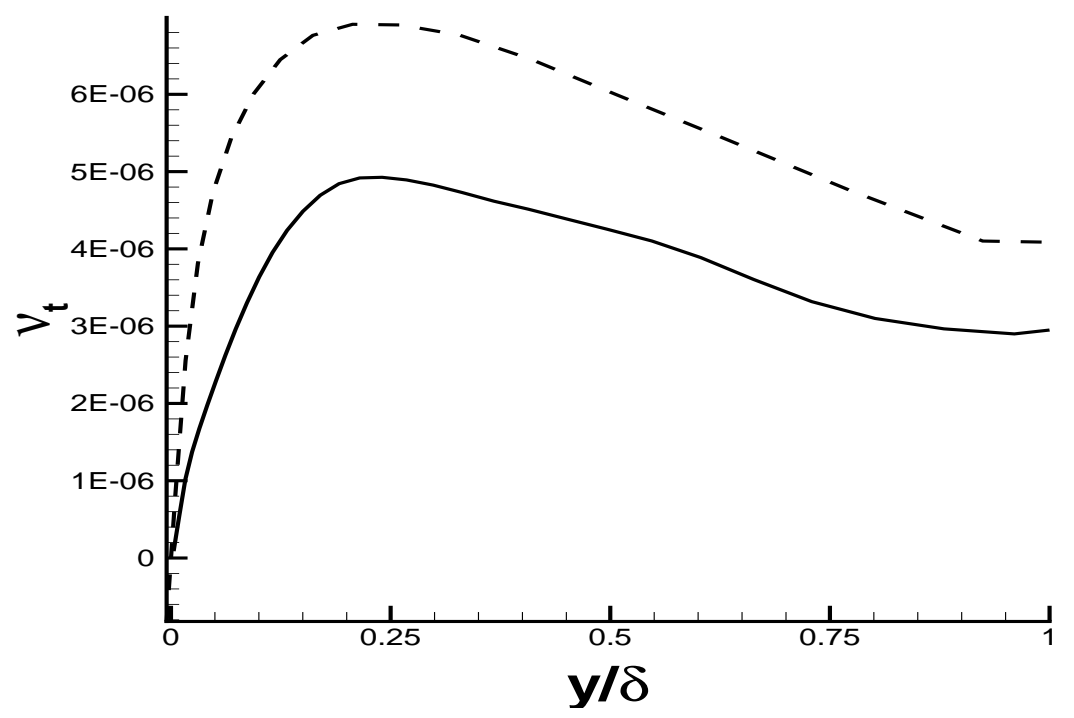

Figure 5.3: The mean turbulence viscosity. $\_$, case $I V ;----$, case $I I$.

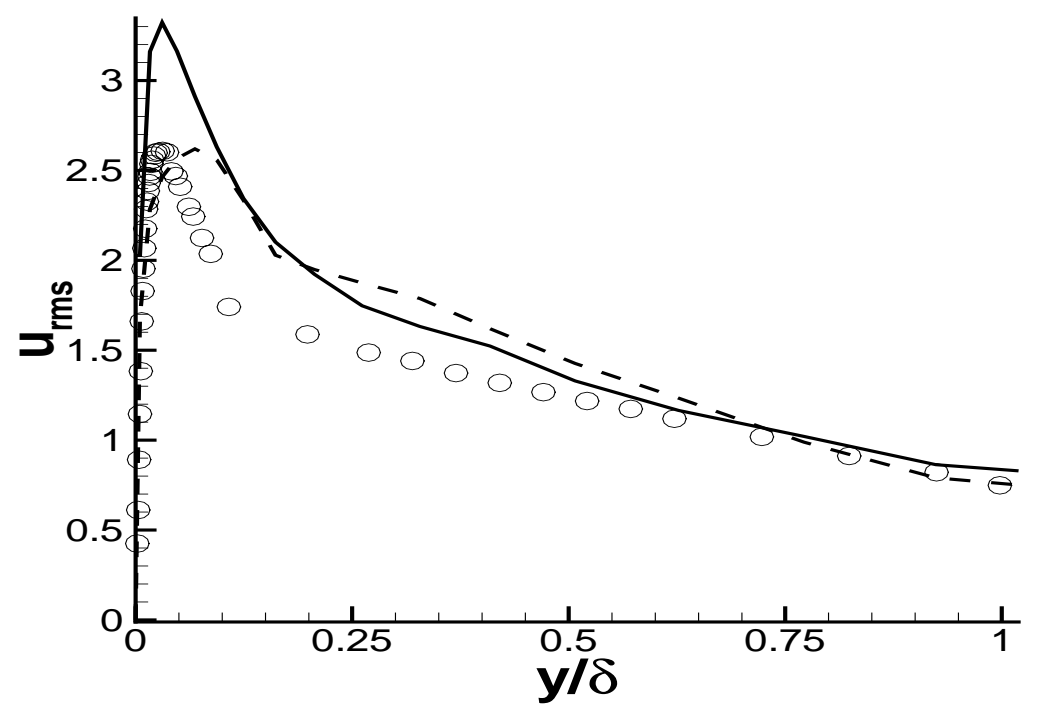

Figure 5.4: The turbulence intensity $u_{r m s} .-\longrightarrow$, case $I I ;----$, case $I$; ०, measurements (Hussain \& Reynolds, 1975). 


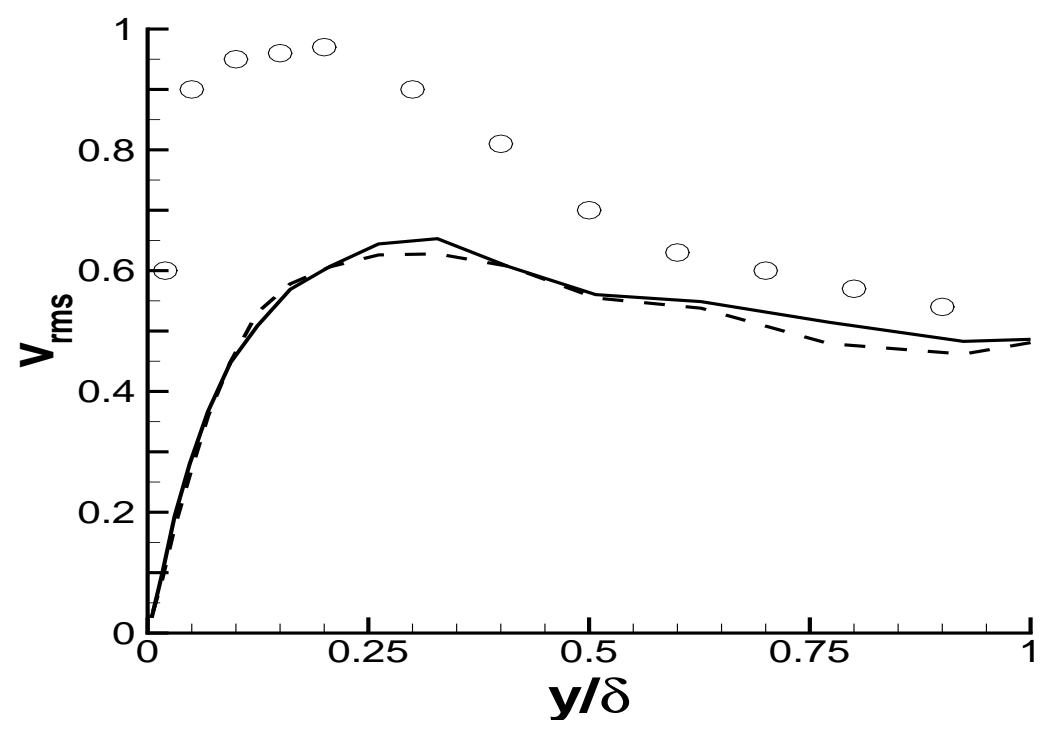

Figure 5.5: The turbulence intensity $v_{r m s} .-$, case $I I$; - - - , case $I$; ०, measurements (Clark, 1968).

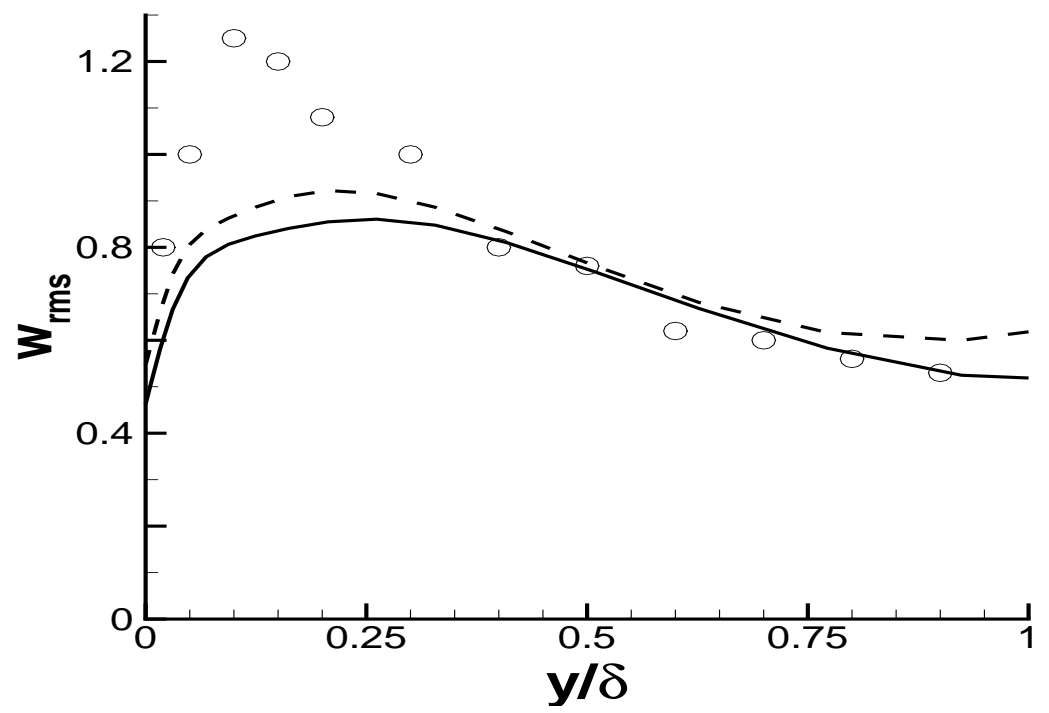

Figure 5.6: The turbulence intensity $w_{r m s} .-\longrightarrow$, case $I I$; - - - , case $I$; 。, measurements (Clark, 1968). 


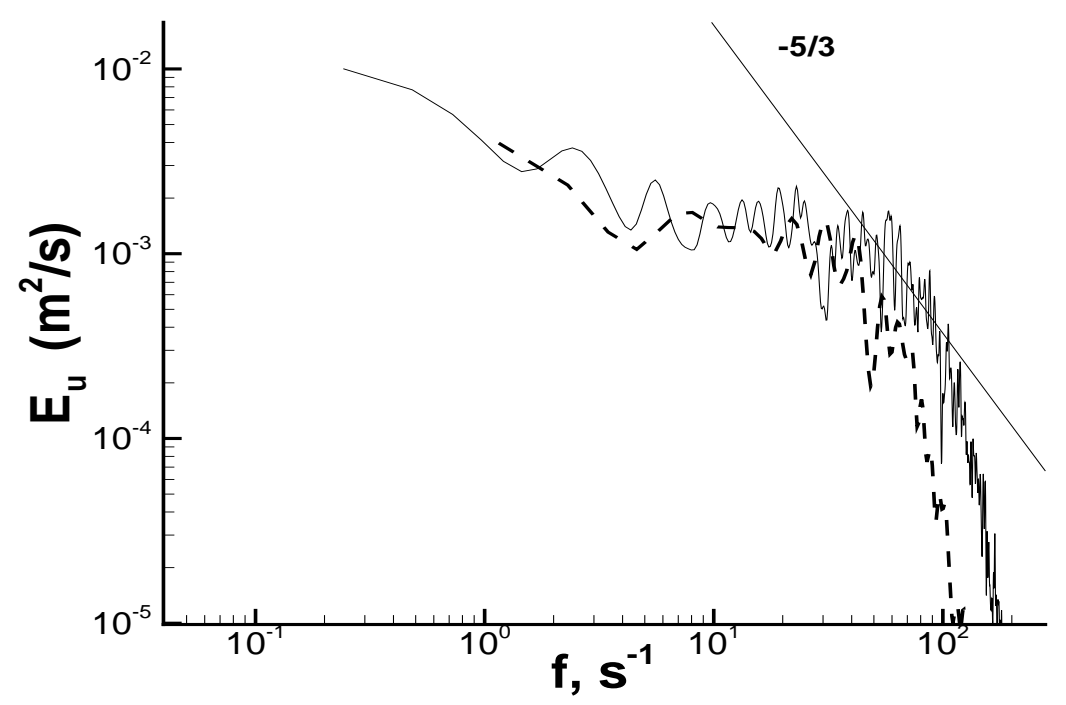

Figure 5.7: The energy spectra. ——, case $I I ;----$, case $I$.

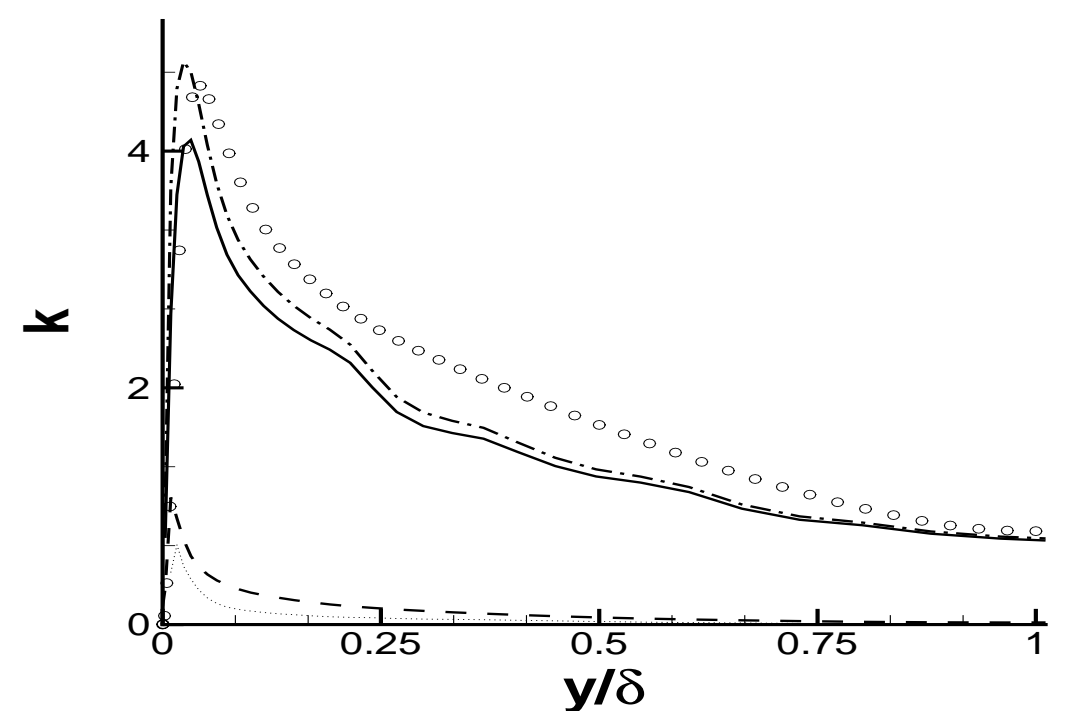

Figure 5.8: The comparisons of the resolved turbulent kinetic energy and subgrid-scale turbulent kinetic energy. ——, resolved $k ;----$, subgrid-scale $k ; \cdots \cdots \cdot$, estimated subgrid-scale $k$ from Smagorinsky model; $-\cdot-\cdot-\cdot$, the total calculated TKE; $\circ$, measurements (Hussain \& Reynolds, 1975; Clark, 1968). 


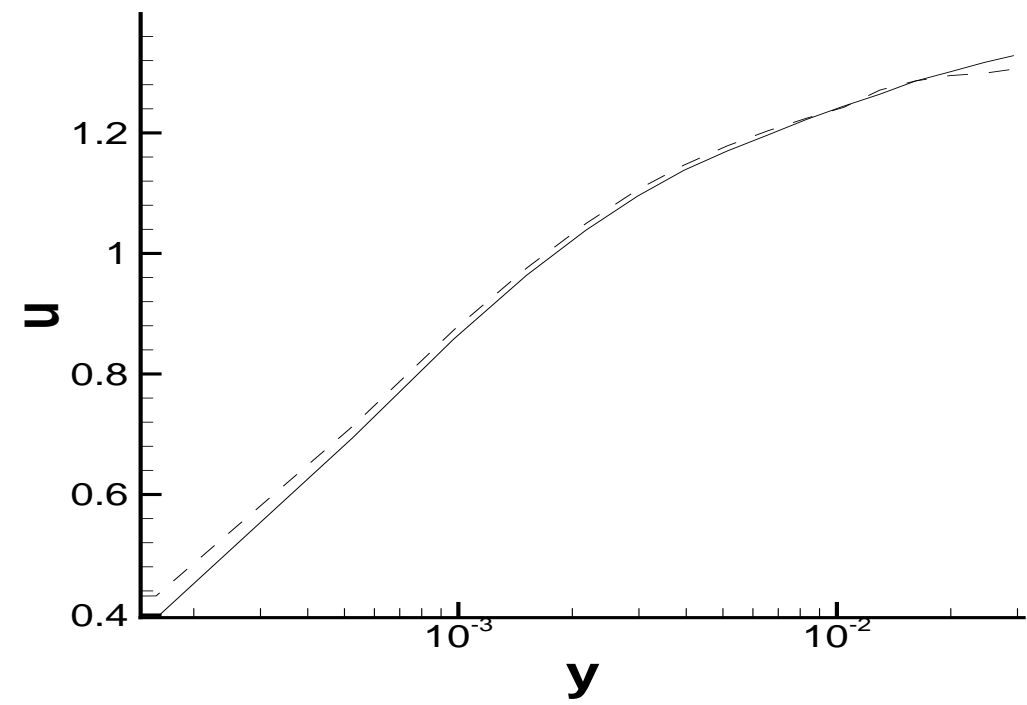

Figure 5.9: The mean streamwise velocity. _—, without backscatter; - - - , with backscatter (ESM).

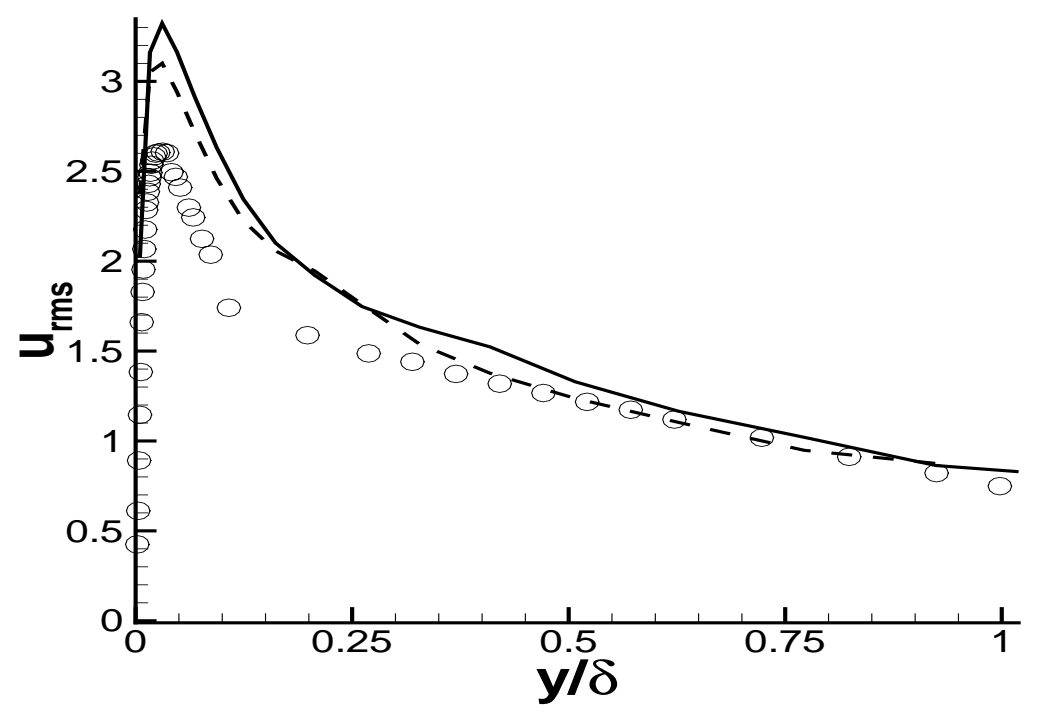

Figure 5.10: The turbulence intensity $u_{r m s} .-\longrightarrow$, without backscatter; - - - , with backscatter (ESM); o, measurements (Hussain \& Reynolds, 1975). 


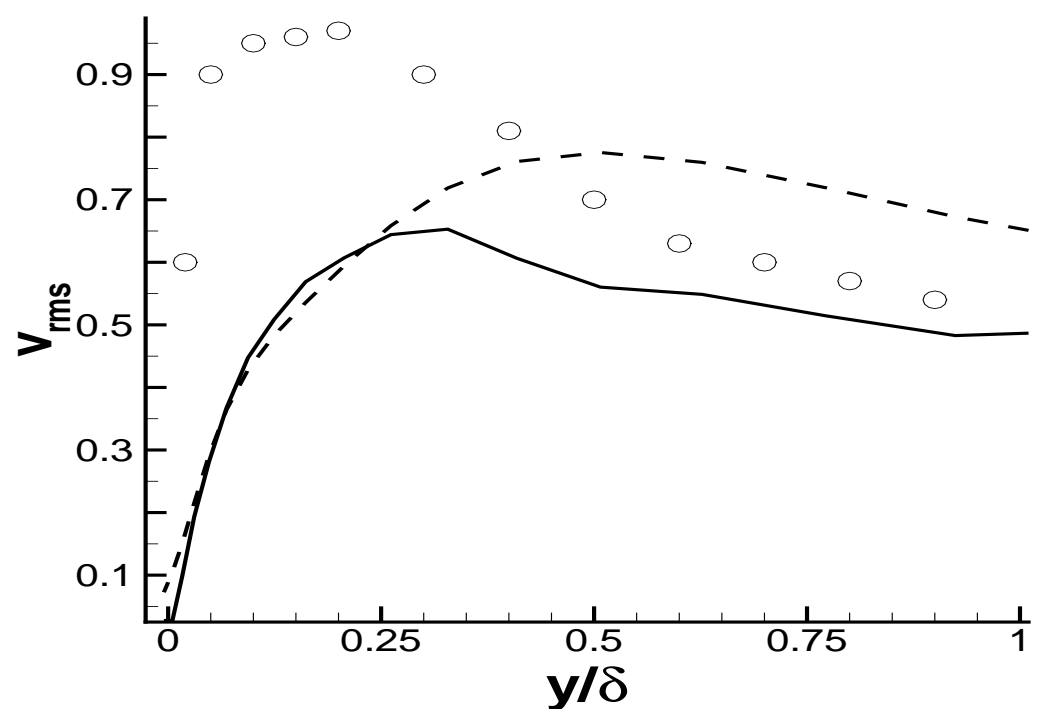

Figure 5.11: The turbulence intensity $v_{r m s}$. - , without backscatter; - - - , with backscatter (ESM); o, measurements (Clark, 1968).

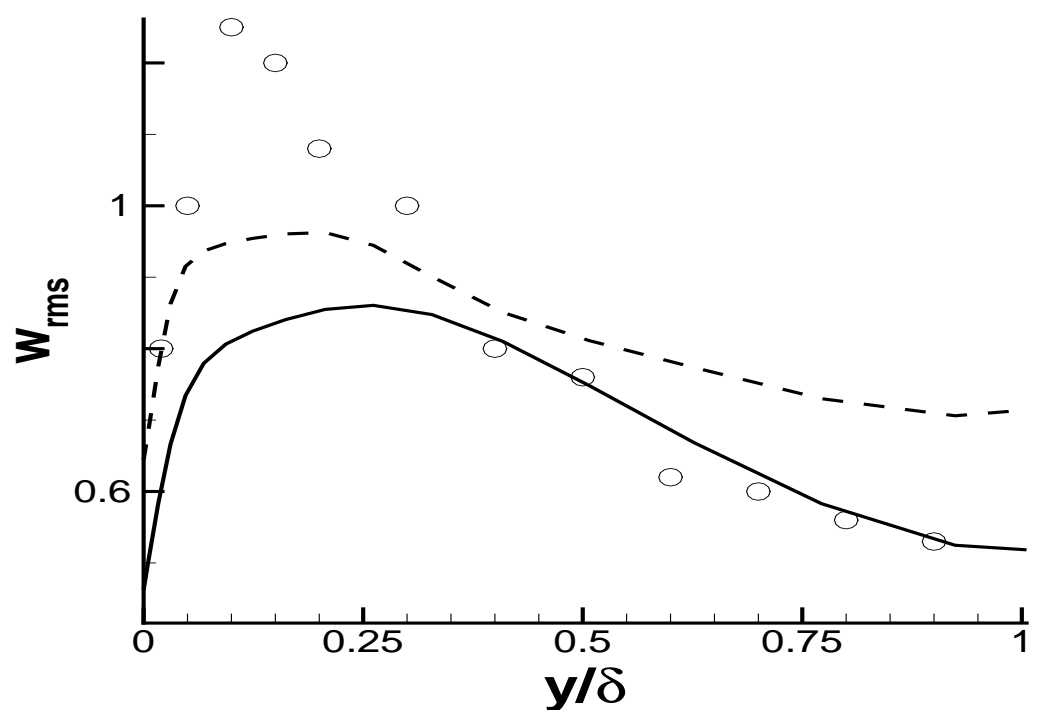

Figure 5.12: The turbulence intensity $w_{r m s}$. - - without backscatter; - - - , with backscatter (ESM); o, measurements (Clark, 1968). 


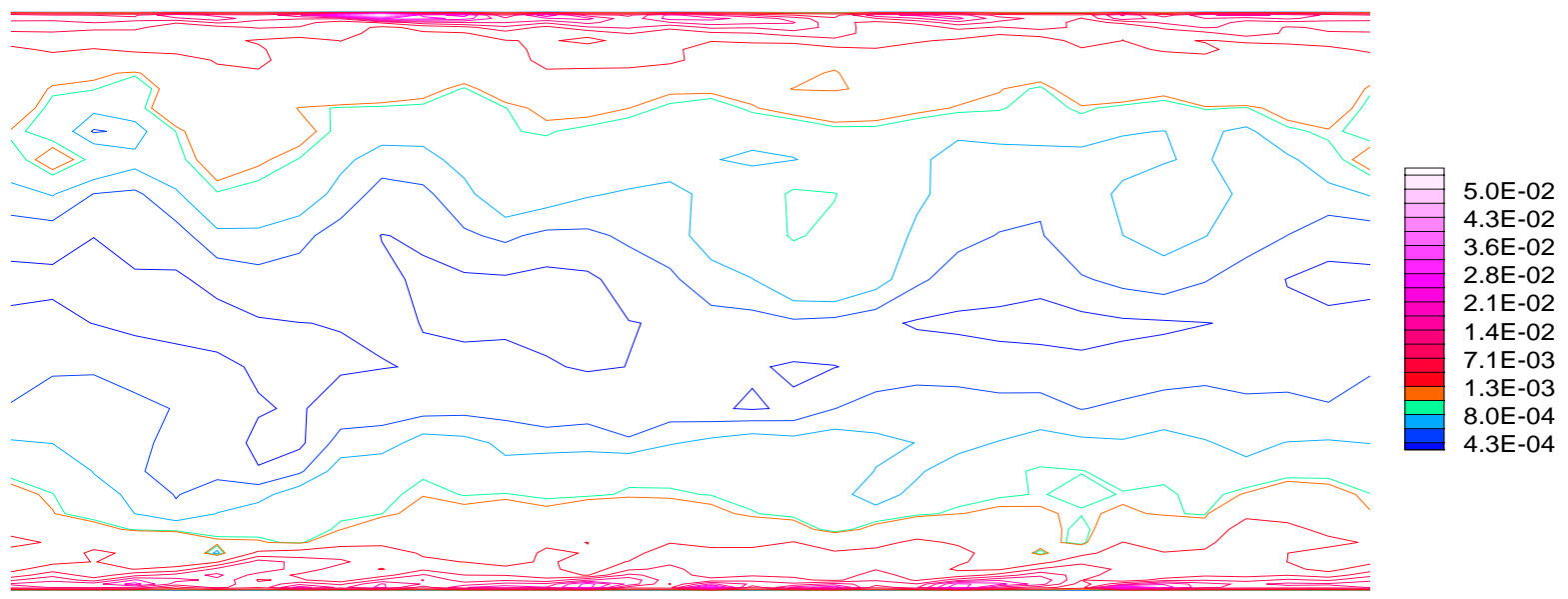

Figure 5.13: Typical subgrid scale turbulent kinetic energy at the center $x y$ plane with backscatter (ESM).

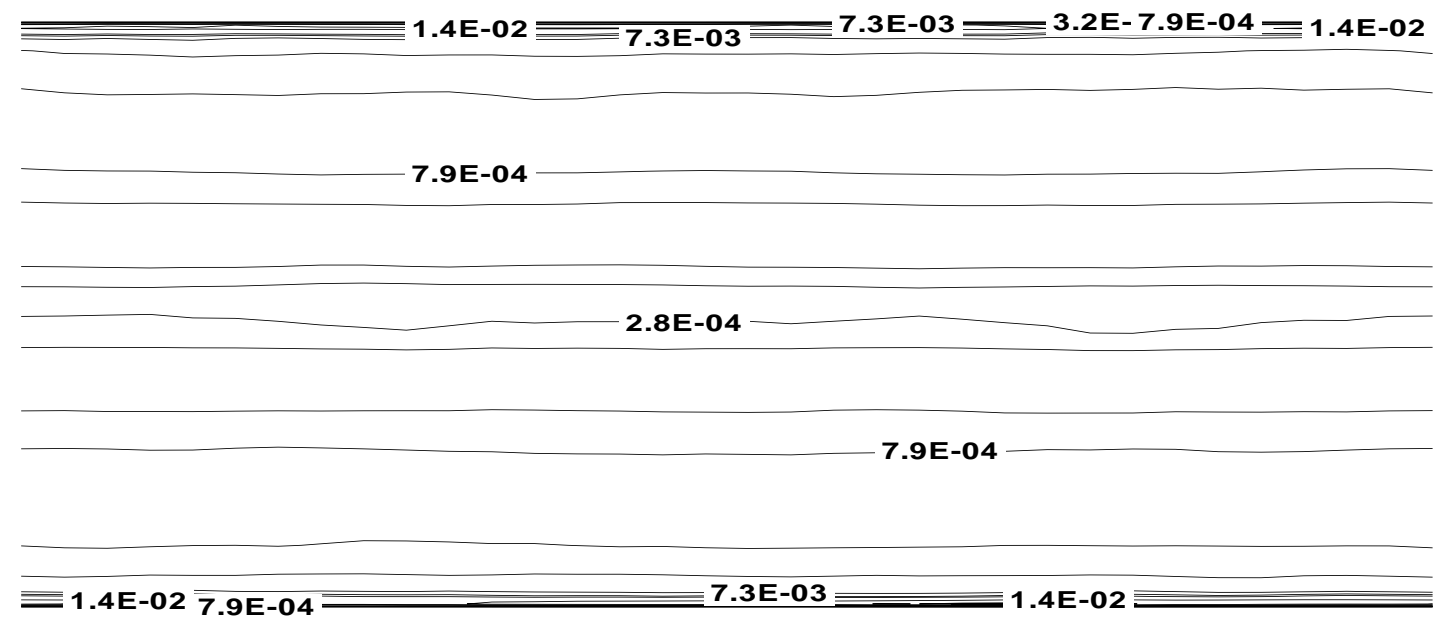

Figure 5.14: Typical subgrid scale turbulent kinetic energy at the center $x y$ plane without backscatter. 


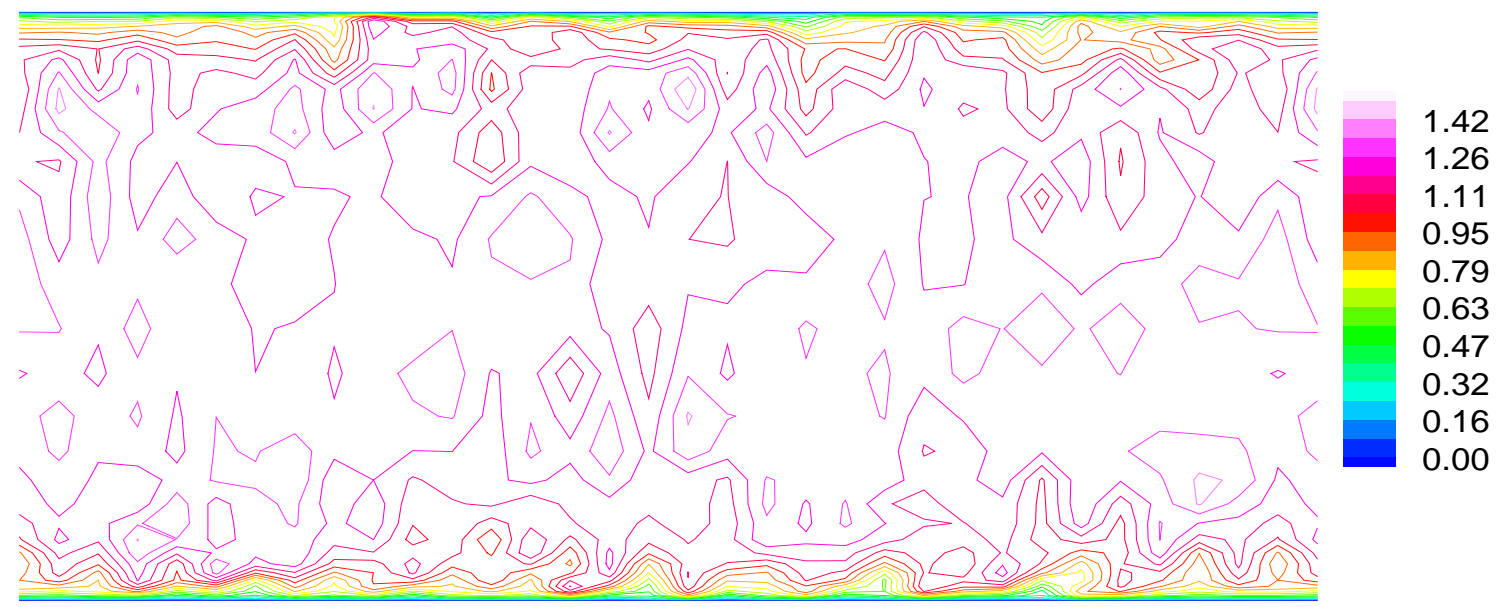

Figure 5.15: Typical streamwise velocity contours at the central $x y$ plane with backscatter (ESM).

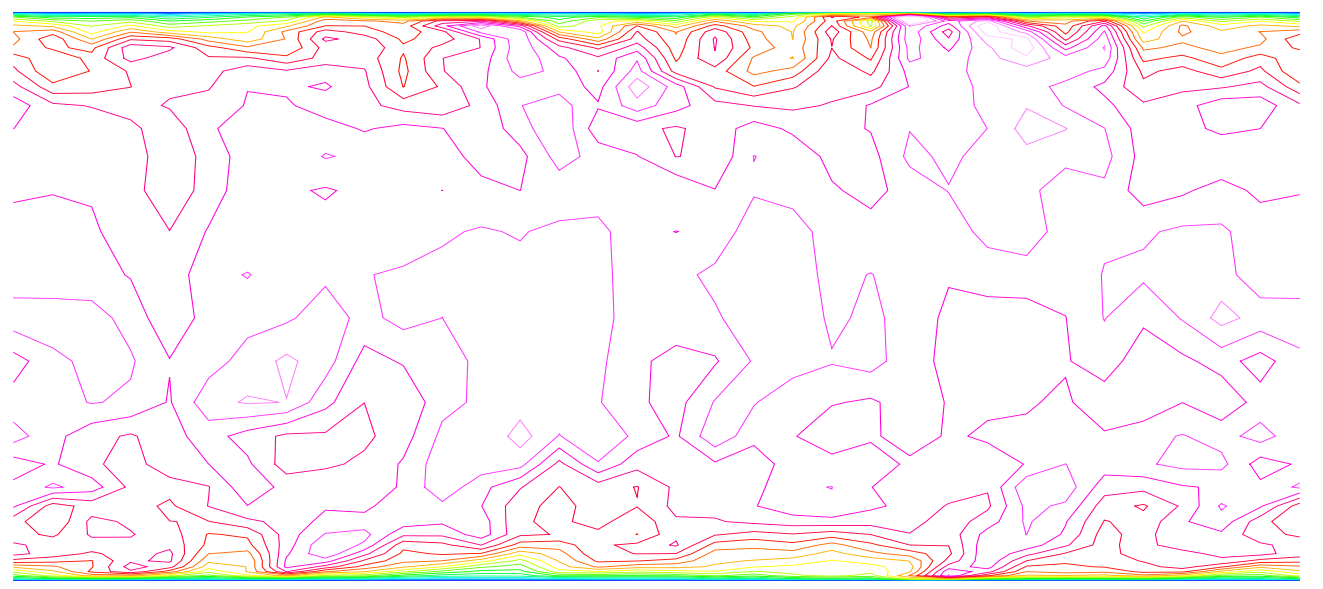

1.42

1.26

1.11

0.95

0.79

0.63

0.47

0.32

0.16

0.00

Figure 5.16: Typical streamwise velocity contours at the central $x y$ plane without backscatter. 


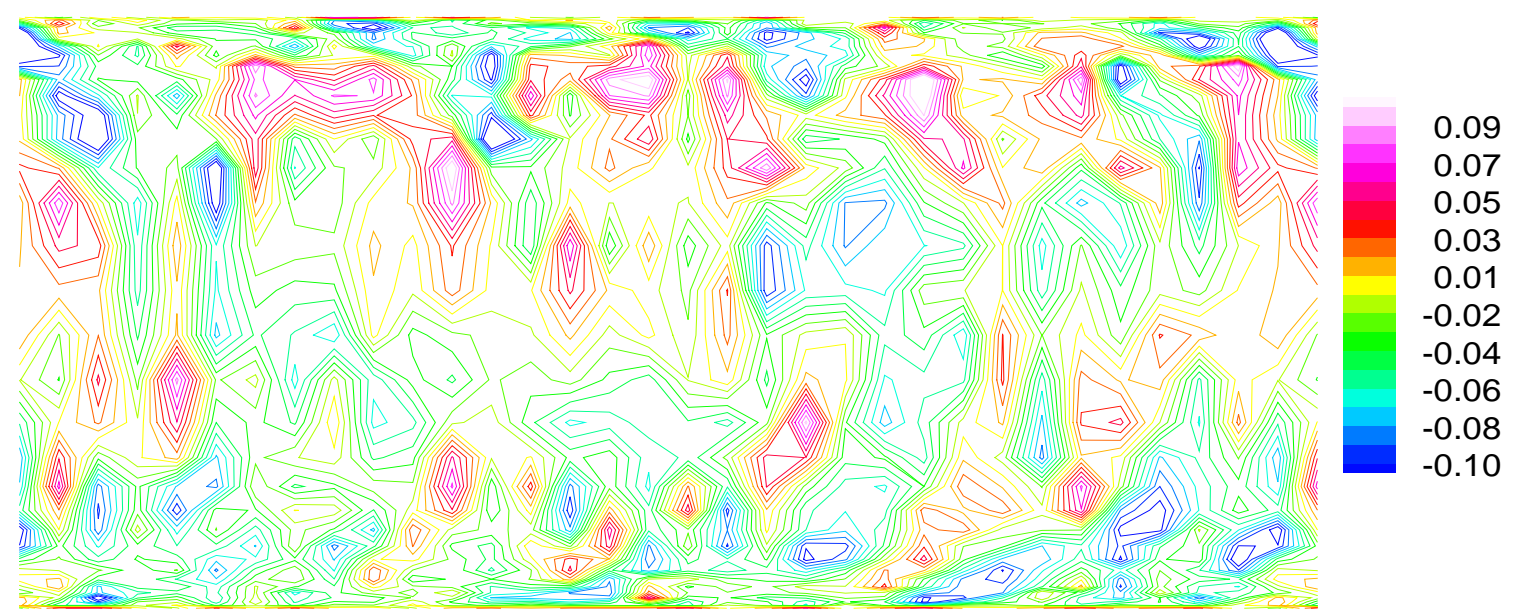

Figure 5.17: Typical spanwise velocity contours at the central $x y$ plane with backscatter (ESM).

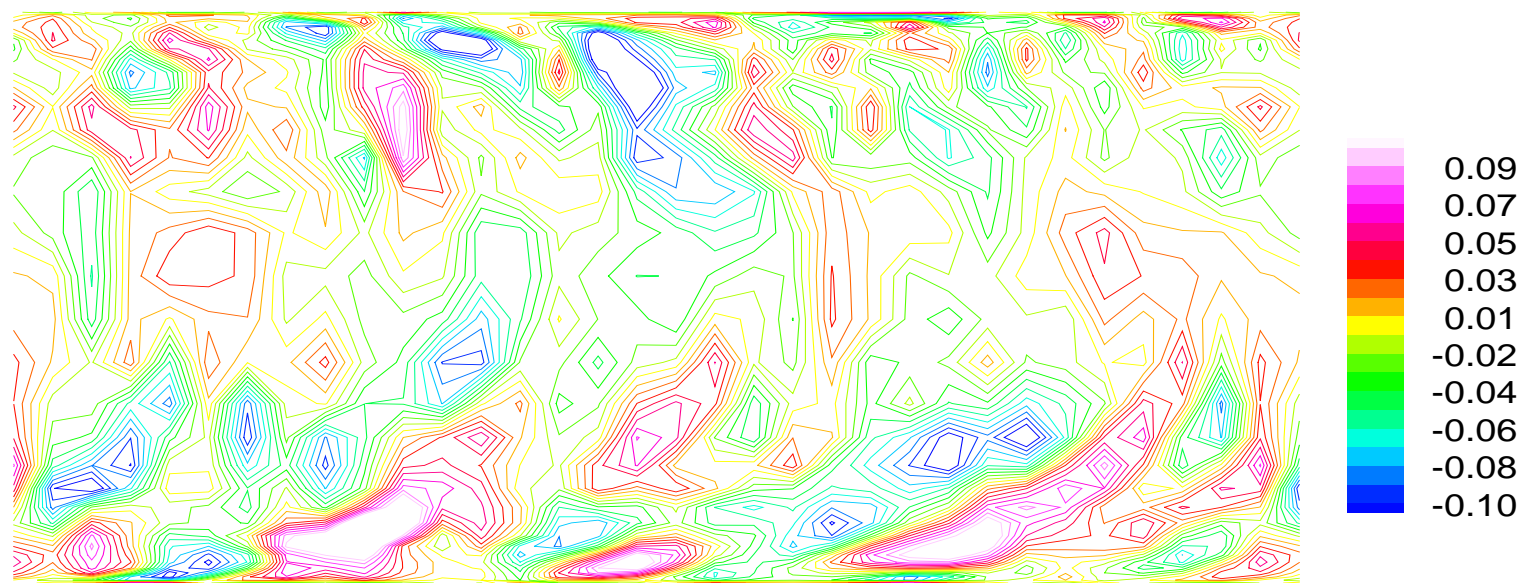

Figure 5.18: Typical spanwise velocity contours at the central $x y$ plane without backscatter. 


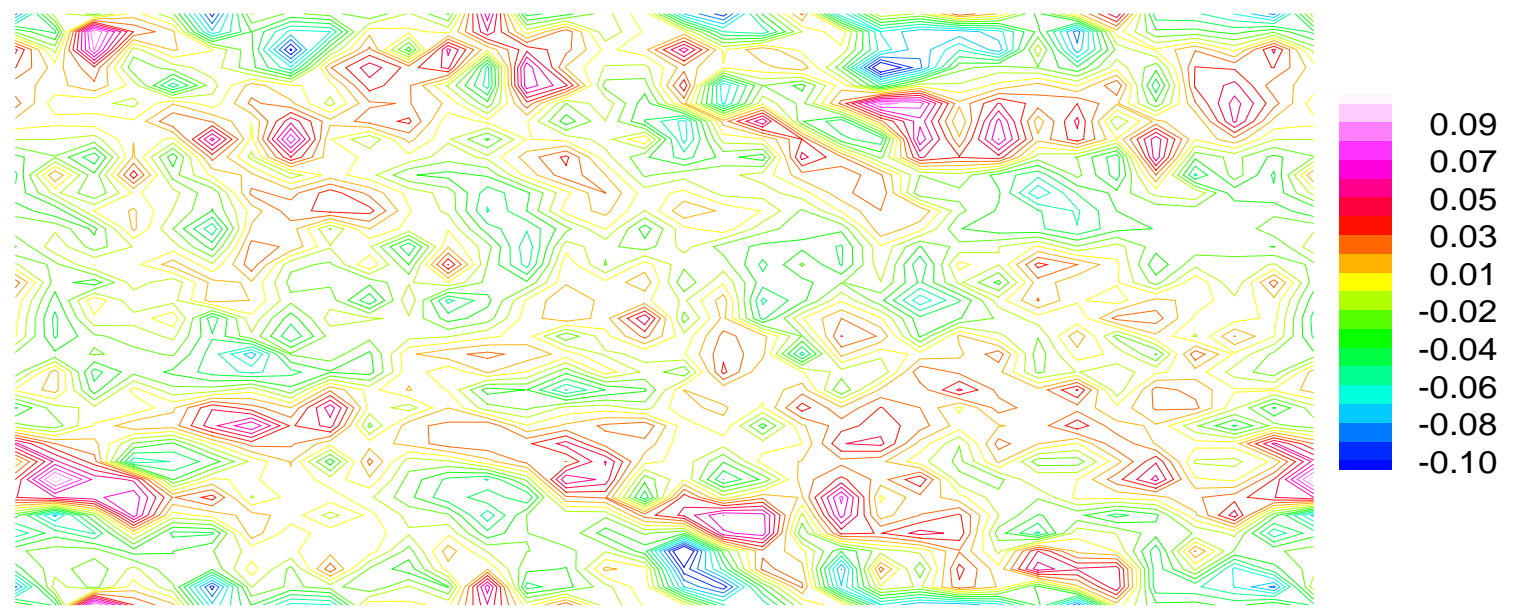

Figure 5.19: Typical spanwise velocity contours on the $x z$ plane at $y / \delta=0.16$ with backscatter (ESM).
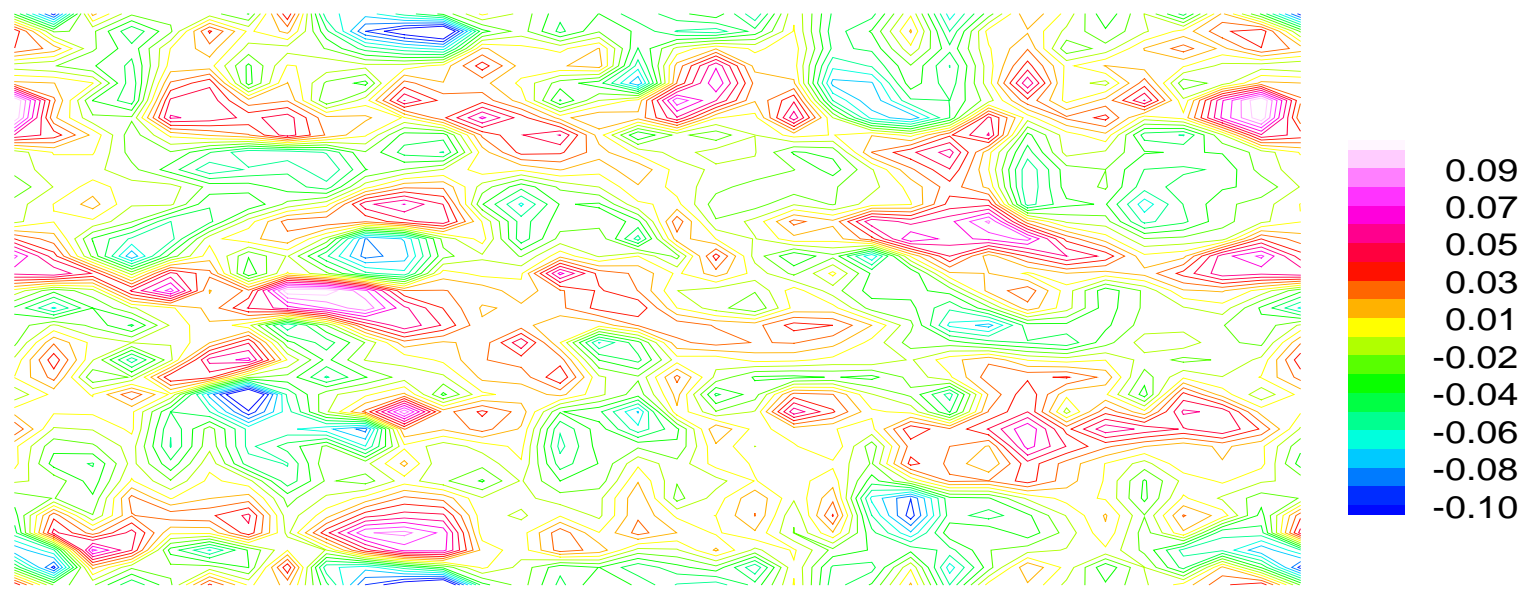

Figure 5.20: Typical spanwise velocity contours on the $x z$ plane at $y / \delta=0.16$ without backscatter. 


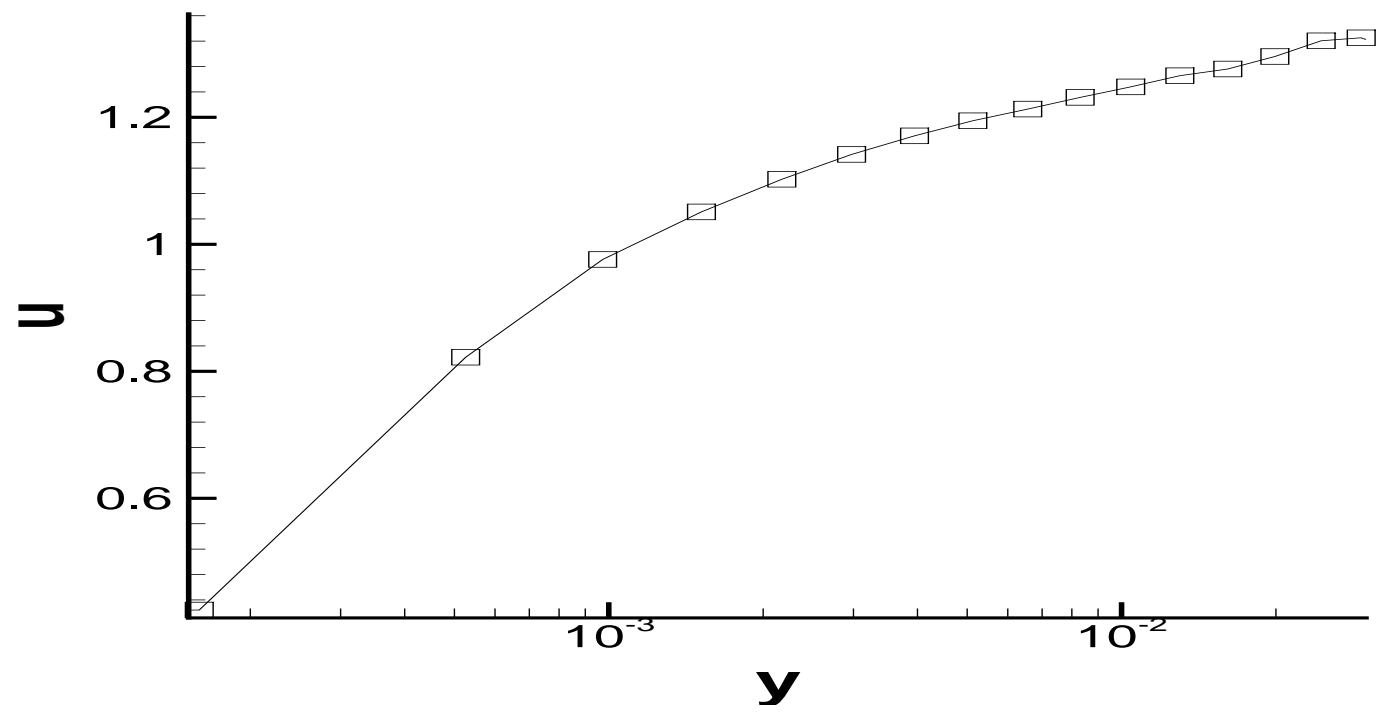

Figure 5.21: The mean streamwise velocity with backscatter (ISM), the logarithm range is shown.

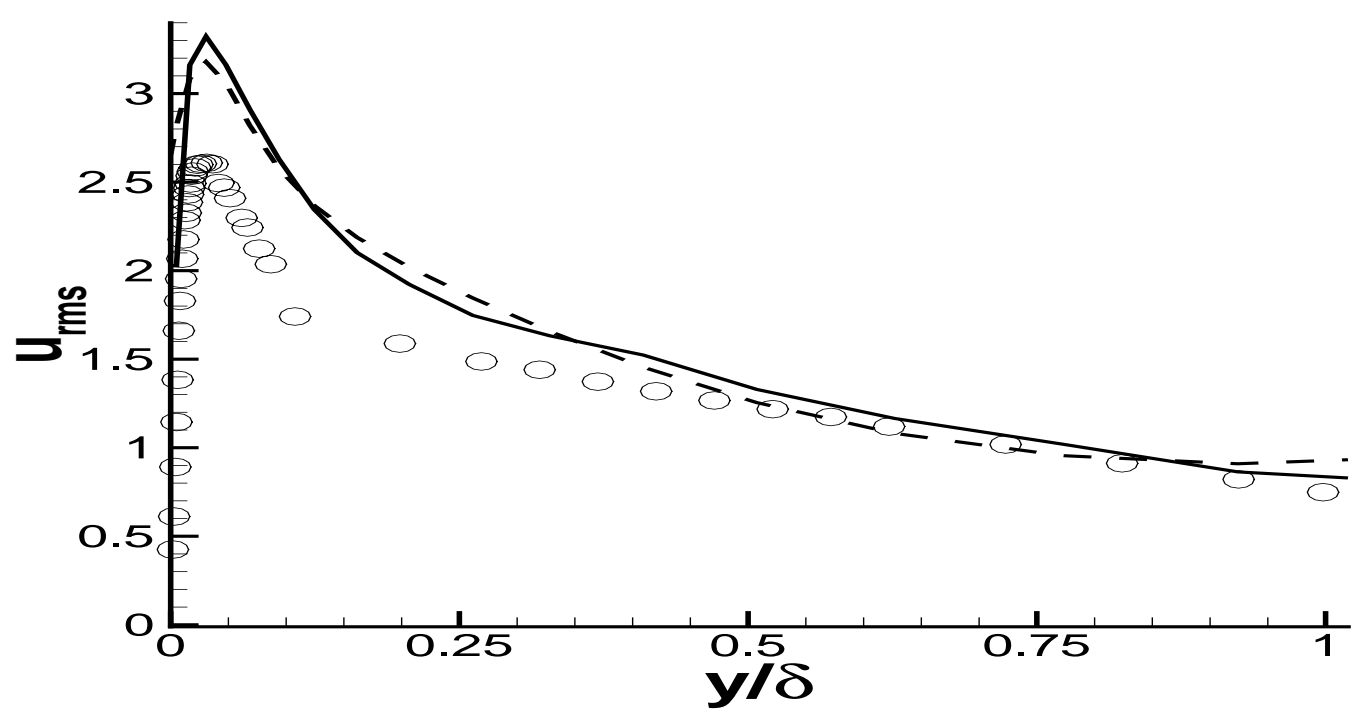

Figure 5.22: The turbulence intensity $u_{r m s}$. - , without backscatter; - - - , with backscatter (ISM); o, measurements (Hussain \& Reynolds, 1975). 


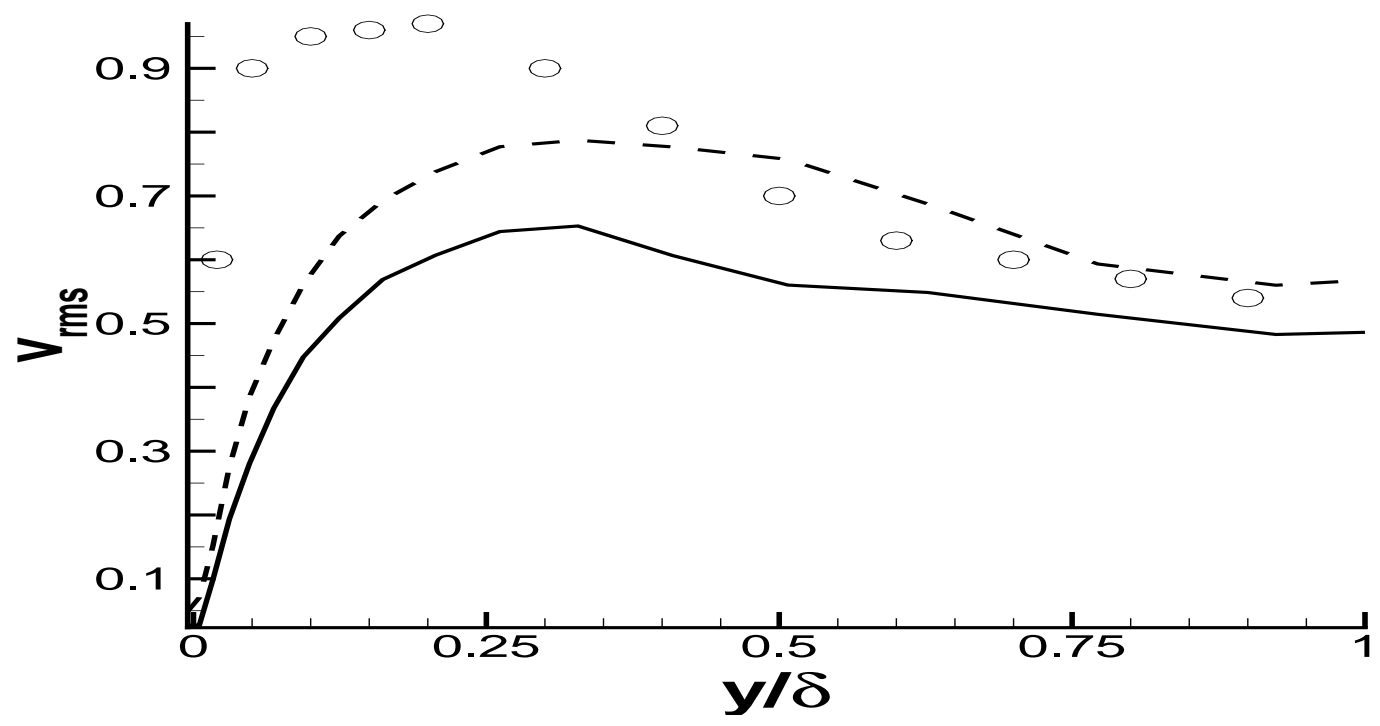

Figure 5.23: The turbulence intensity $v_{r m s}$. - - without backscatter; - - - , with backscatter (ISM); o, measurements (Clark, 1968).

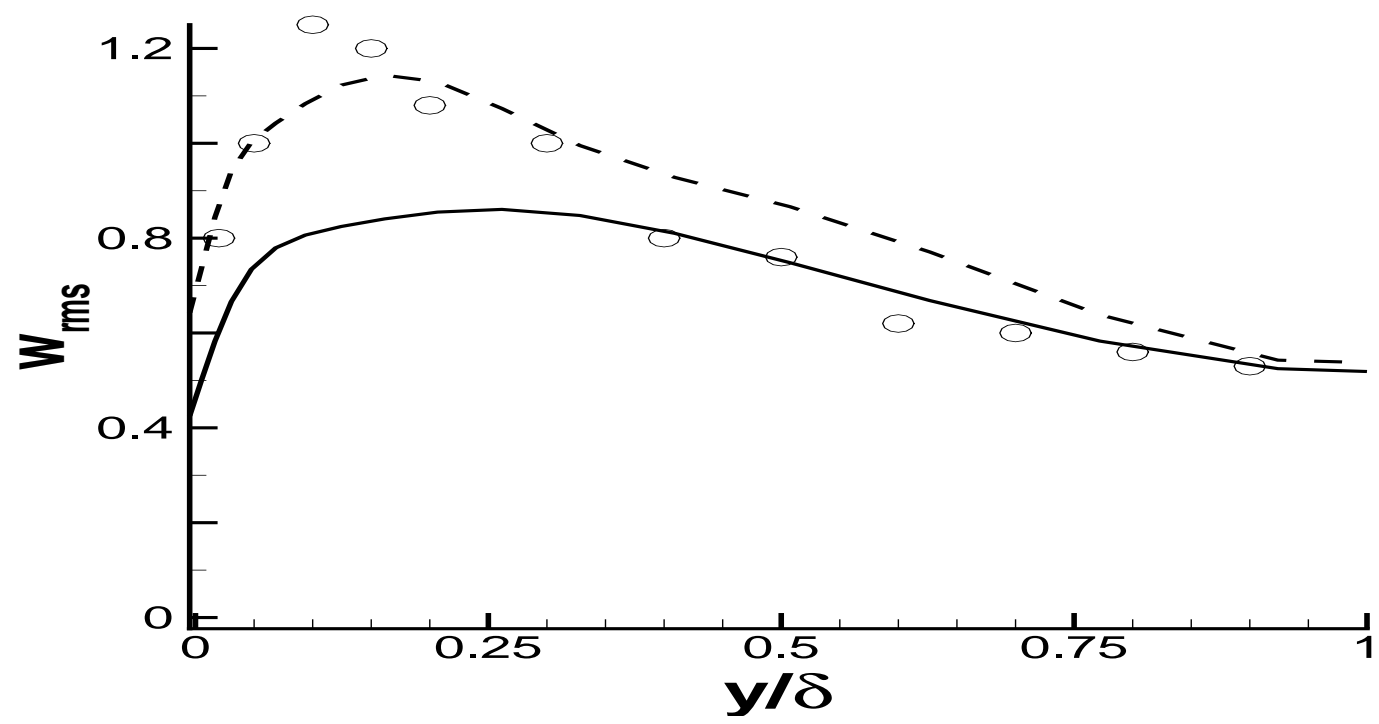

Figure 5.24: The turbulence intensity $w_{r m s}$. - - without backscatter; - - - , with backscatter (ISM); ○, measurements (Clark, 1968). 


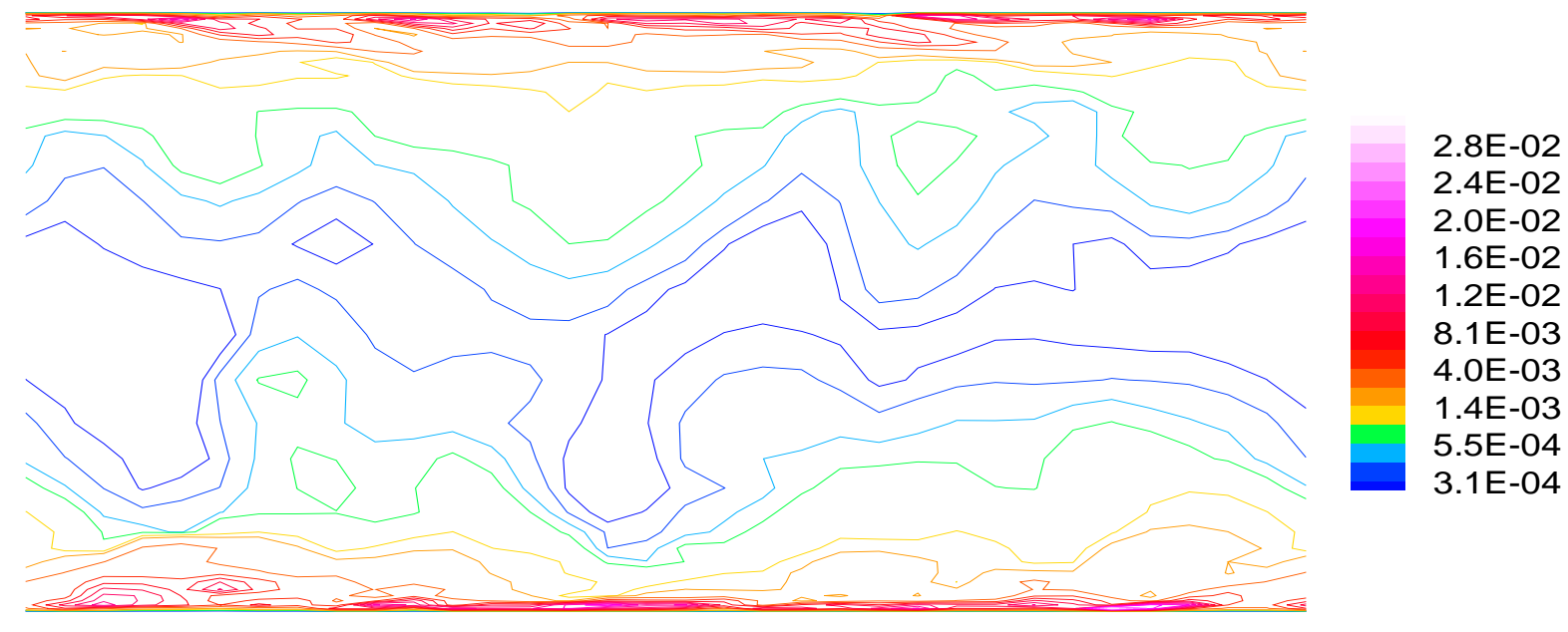

Figure 5.25: Typical subgrid scale turbulent kinetic energy at the central $x y$ plane with backscatter (ISM).

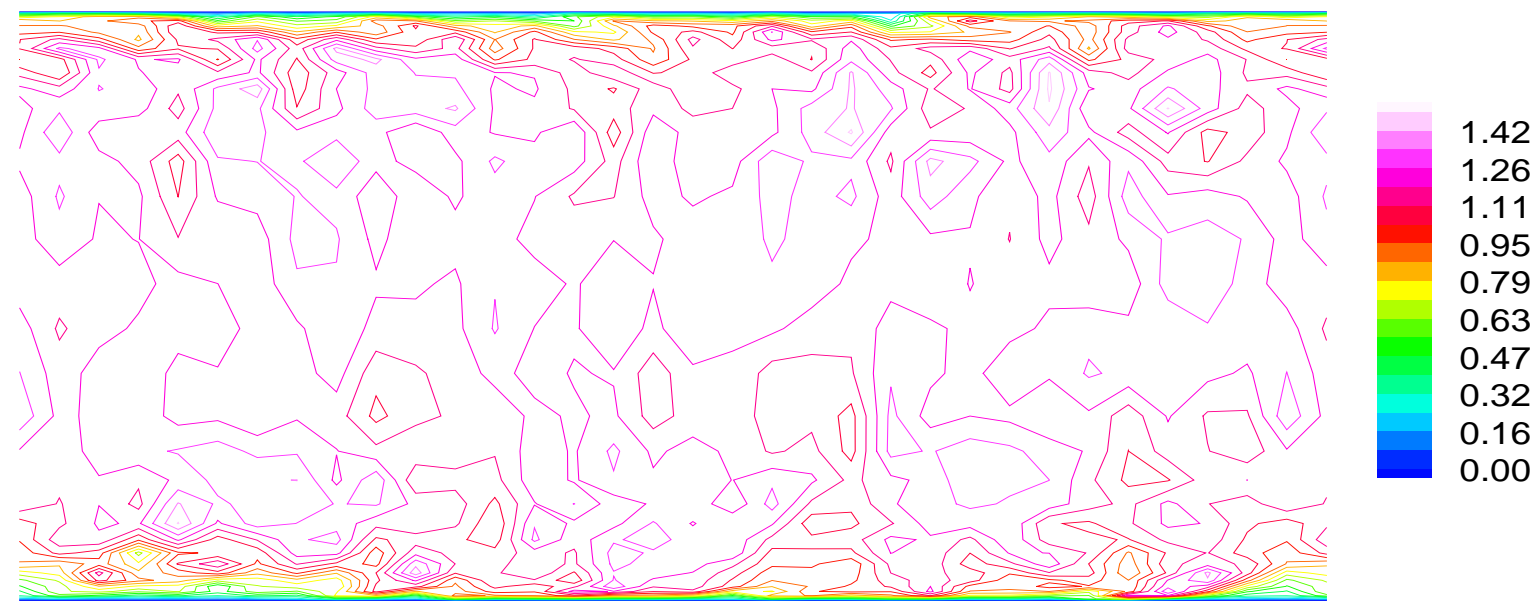

Figure 5.26: Typical streamwise velocity contours at the central $x y$ plane with backscatter (ISM). 


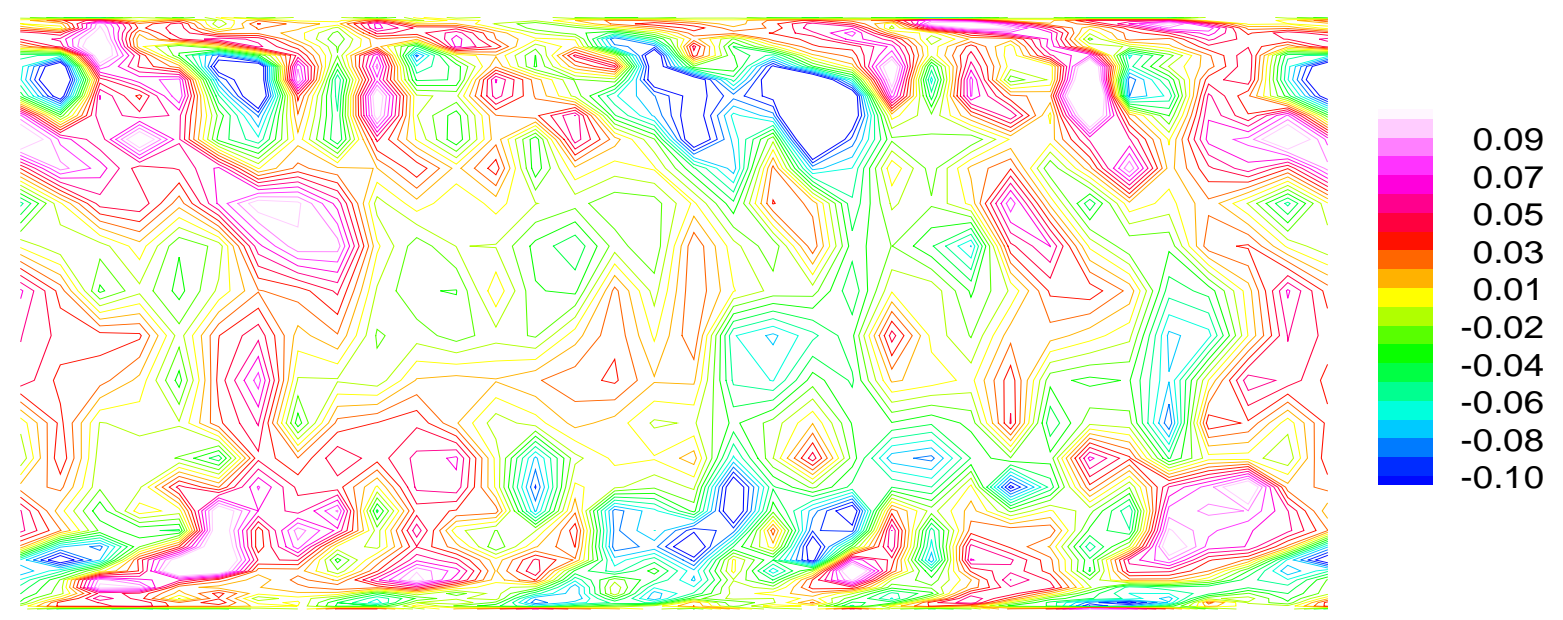

Figure 5.27: Typical spanwise velocity contours at the central $x y$ plane with backscatter (ISM).

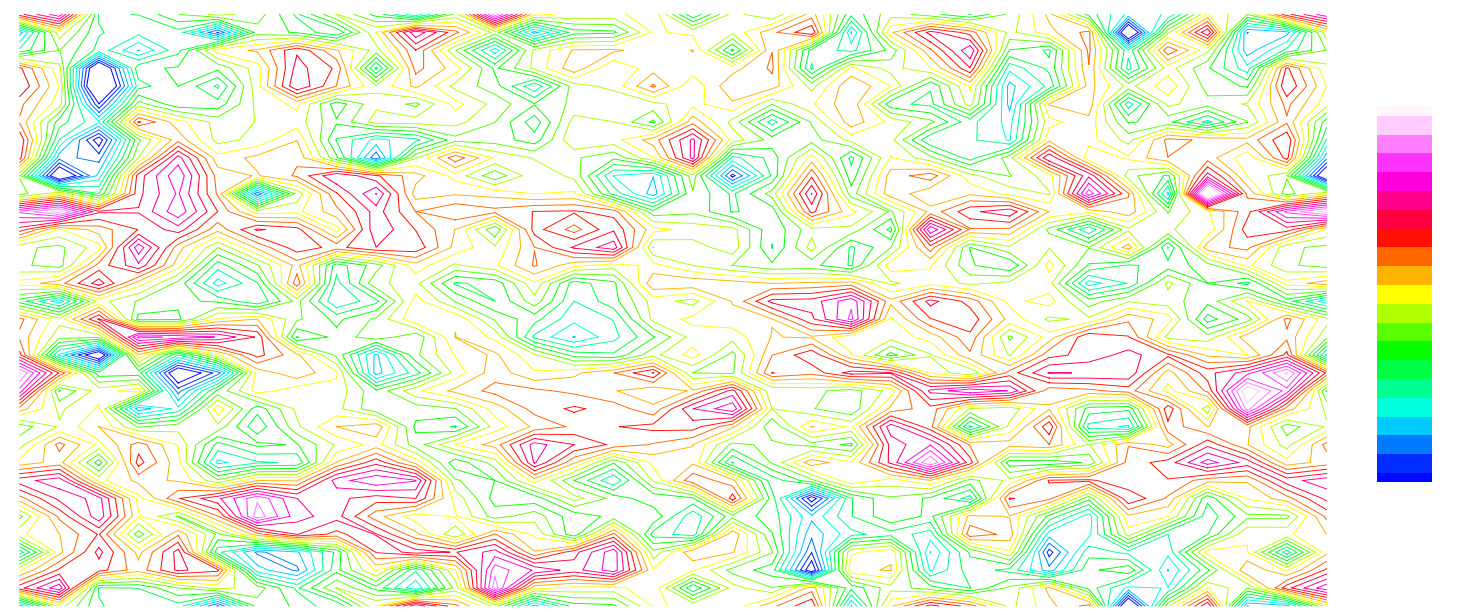

Figure 5.28: Typical spanwise velocity contours on the $x z$ plane at $y / \delta=0.16$ backscatter (ISM). 


\section{Chapter 6}

\section{Applications to Flat Plate Wake}

\subsection{Introduction}

The primary goal of this research is to study the turbulent flow in the ship wake. Due to the lack of measurement or DNS data for the turbulent ship wake flows, to the best of author's knowledge, it is necessary to test the techniques described in the previous chapters by applying them to a well documented similar, but simpler, flow. The flat plate wake is thought to be a proper case.

In line with the present approach of simulating high-Reynolds number wake flows without incorporating the body itself, the LES simulations started from a plane located behind the plate in the wake region (Fig. 6.1). In this way solving a complex boundary layer problem around the object, e.g. flat plate, was avoided. The inflow boundary is generated using the RFG method described above with input from experiments (Ramaprian et al., 1981), including mean velocity $U_{m}$, the turbulence intensities $u_{r m s}, v_{r m s}, w_{r m s}$, and the turbulent shear stress $\overline{u v}$. Thus the realistic inflow boundary conditions, which include the turbulence characteristics created by the plate, are provided. This is an important factor for accurate and efficient LES. 


\subsection{Details of Simulations}

The length of the flat plate is $1.829 m$ (Ramaprian et al., 1981). The inflow boundary is located at $19.5 \mathrm{~mm}(x / L=0.01)$ behind the rear edge of the plate. The computational domain size is $1.0 m \times 0.2 m \times 0.6 m$ in $\mathrm{x}, \mathrm{y}$ and $\mathrm{z}$ direction, respectively. Two different grid numbers have been used. One of these is $82 \times 50 \times 50$, which is the primary grid in this case. Another is $258 \times 66 \times 50$, which is used to test the sensitivity of the grid resolution in LES. A non-uniform grid is used in both $\mathrm{x}$ and $\mathrm{y}$ directions with stretching not exceeding three

percent. Again, in this study, x represents streamwise direction, y represents vertical and $\mathrm{z}$ represents spanwise direction, respectively. Neumann boundary condition (free gradient) is applied for the outflow. Symmetry boundaries are used in vertical direction and periodic boundaries are used in the spanwise direction. Unless particularly pointed out, a central difference scheme is applied.

\subsection{Inflow Boundary}

The time-dependent inflow boundary conditions were re-constructed using RFG and the experimental measurements. Because the inlet plane is very close to the flat plate, it still keeps the features of boundary layer. Thus the length scale and the time scale can be calculated by using similar methods as those described in Section 4.4. Fig. 6.2 shows the $u, v$, and $w$ velocity time histories at one point at the inlet plane. The velocity fluctuations closely resemble the real turbulence except that it has a weak periodic behavior because sinusoidal functions were used to generate these signals (Eq.4.4).

Fig.6.3 to Fig.6.6 present the comparison of the turbulence statistics between the simulations and the measurements at the inlet plane. The agreement is very good except very near the centerline of the wake. This is because the grid is relatively coarse in this region, which leads to the smoothing of the very sharp gradients. The overall difference is under 
$1 \%$. It should be noted that the agreement is good because the comparison is given for the inlet plane where the RFG procedure was designed to reproduce the turbulence quantities exactly.

\subsection{Instantaneous Flow Field}

Fig. 6.7 shows the contours of the streamwise vorticity at different cross sections. As can be seen, with the increasing of $x$, the size of the turbulent eddies increase while their vorticity (i.e. their strength) decrease which means the turbulence is decaying. Strong anisotropic structures are notable. In Fig.6.8, the velocity time histories are shown for different points along the wake. From these pictures it can be seen that both the amplitude and the frequency of the velocity fluctuations decrease (decaying turbulence) along the streamwise direction which is consistent with the behavior of a turbulent wake. Additionally, the grids are becoming coarse with the increasing of $x$ which might have smeared out part of the velocity fluctuations. To support these conclusions, the energy spectrum at the corresponding locations have been calculated. Fig.6.9 shows the energy spectrum at the inflow boundary. A sharp cut-off exists at the wave length 0.001 which matches the length scale that was selected for RFG. Fig.6.10 and Fig.6.11 show the energy spectra at $x=0.16$ and $x=0.53$, respectively. It is seen from these figures that a good portion of the inertial range is captured. With the grid becoming coarser farther downstream in the wake only large wave lengths can be resolved. The predicted anisotropy is also noteworthy.

The spanwise vorticity contours are shown in Fig.6.12(a). Large coherent structures are clearly visible. These structures are similar in appearance to the Karman vortex street because they seem to be comprised of vortices of alternating sign of vorticity. This is similar to the experimental visualization (Fig.6.12(b)) of Wygnanski et al. (1986). As they explained "Neither the varicose mode, which requires that the vortices appear in pairs distributed symmetrically about the centerline, nor the sinuous mode, which requires vortices 
whose center coincides with the centerline, dominate this flow". Similar structures can be seen in the simulations (Fig.6.12(a)).

\subsection{Mean Flow Quantities}

It is believed by many authors (Nakayama and Liu, 1990; Ramaprian and Patel, 1982; Wygnanski et al., 1986; Andreopoulos and Bradshaw, 1980) that in the near wake the meanvelocity in both streamwise and transverse directions has a log-law feature as in the wall boundary layer, although Nakayama and Liu (Nakayama and Liu, 1990) claimed that this behavior is Reynolds number dependent while the others have the opposite point of view. If such a feature exists, then $U_{c}^{+} \equiv U_{c} / u_{\tau}$ versus with $x^{+} \equiv x u_{\tau} / \nu$ could be described by the logarithmic function

$$
U_{c}^{+}=A \log _{10} x^{+}+B
$$

where $U_{c}$ is the streamwise mean velocity at the center line, $u_{\tau}=0.853 \mathrm{~m} / \mathrm{s}$ is the friction velocity of the boundary layer at the trailing edge, $x$ is the distance from the plate edge, $\nu$ is the kinetic viscosity and $A$ and $B$ are constants which are 4.65 and 0.7 (Andreopoulos and Bradshaw, 1980), respectively.

In the transverse direction (direction normal to the plate), the logarithmic mean velocity profile (if it exists) can be expressed (Nakayama and Liu, 1990) as

$$
U^{+}=\frac{1}{\kappa} \ln y^{+}+5.2
$$

where $U^{+} \equiv U / u_{\tau}$ and $y^{+} \equiv y u_{\tau} / \nu . \kappa$ is the Von Karman constant taken as 0.41 .

Fig.6.13 and Fig.6.14 show the comparisons of the present simulations with experiments (Ramaprian and Patel, 1982). The predictions are in good agreement with experiments. The predicted mean velocity along the $x$ - direction has a small amplitude long wave 
oscillation. This phenomenon may be due to a weak vortex shedding which could exist because only the statistical analysis at one line instead of the whole center plane was performed. In Fig. 6.13, the results from the QUICK scheme is also presented. There is not much difference between the QUICK scheme and CD scheme results. It has to be pointed out that when QUICK scheme is applied, no SGS model is used.

The ergodicity of the flow field was tested and shown in Fig. 6.15. The results of time average was taken at the center line on the middle plane, while space average was taken by averaging the instantaneous velocities of the same plane along the spanwise direction. The difference is significant especially at the downstream locations. It is noticed that if the mean of the time averaged and space averaged results are taken, the simulations could agree with the measurements even better.

\subsection{Reynolds Stresses}

Figures 6.16 to 6.19 show the computed Reynolds stresses along $y$-direction at different $x$ locations in comparison with the experimental results. The agreement is good although more quantitative studies would be appropriate. Near the very beginning of the wake, most of the fluctuations can be captured. Later in the flow field, the percentage of the resolved turbulence is becoming small due to the coarser grids. It can be noted that the values of both $v^{\prime}$ and $\overline{u v}$ at the second $\mathrm{x}$-station are higher than those at the first $\mathrm{x}$-location $(x=31.75 \mathrm{~mm})$. This effect has been investigated and discussed by Nakayama and Liu (1990) where they attribute it to a near wake phenomenon. Figure 6.20 shows the decay of the turbulent kinetic energy along the center line of the wake. In the near wake most of the turbulent kinetic energy has been captured. Also, it is noted that the LES results with a finer grid are closer to the experimental results. 


\subsection{Sensitivity to Grid Resolution}

Up to now, all the simulations were based on the same grid size, of $82 \times 50 \times 50$. Obviously this is relatively coarse for LES of the flat plate wake. Although some encouraging results have been obtained, in the coarse grid range a considerable difference between simulations and experiments still exists. If the proposed approaches are appropriate, then with the refining of the grid size, more turbulence should be resolved. To test this hypothesis, a finer grid of $258 \times 66 \times 50$ was utilized. All the other conditions were kept same as in the previous simulations.

Fig. 6.20 presents the fine grid solution of the resolved turbulent kinetic energy $(k)$ along the center line. It can be clearly seen that with the refining of the grid size, the solution has been significantly improved. Again, the oscillations are because of the nonsufficient averaging (time averaging only at one line). The Reynolds stresses are also shown in Figures 6.21 to 6.24. Only the comparisons in the far wake are provided here, as having been demonstrated before, the solution in this area is poor when a coarse grid was applied. All the stresses in the central region of the wake have been enhanced by using fine grids. However, away form the centerline, the predictions become worse. This is not surprising because in the vertical $(y)$ direction a relatively coarser grid has been used.

\subsection{Effects of SGS models}

The one equation model proposed in Chapter 5 was applied to the the flat plate wake simulation. The grid number is still $82 \times 50 \times 50$.

Fig. 6.25 shows the comparisons of the turbulent kinetic energy (TKE) along the streamwise direction (the center line of the wake) between the measurements and the simulations. When one equation subgrid scale model was used, the backscatter energy was not included in the simulation. The resolved TKE increases significantly at the beginning, 
much more than that when Smagorinsky model was used. It is not clear to the author why this happens. As the wake develops downstream, the resolved TKE comes much closer to the measurements than that of Smagorinsky model. The subgrid-scale TKE is also presented in this figure. Generally, the summation of the resolved TKE and subgridscale TKE is slightly smaller than the TKE of the measurements. However, considering the uncertainty in the measurements, the agreement between the two is very good. Similar comparisons along the vertical direction at $x=590.55 \mathrm{~mm}$ is also presented in Fig. 6.26. Again, when one equation model was used, the resolved TKE is much higher than that of Smagorinsky model, and the total TKE agrees well with that of the measurements.

The Reynolds stresses along the vertical direction at $x=590.55 \mathrm{~mm}$ are shown in Figures 6.27 to 6.30 . With one equation model being used, only $u$ ' is notably improved. All the other stresses $\left(v^{\prime}, w^{\prime}, u v\right)$ are similar to those when Smagorinsky model was used. The reason is that at this location, the cell size $\Delta x(0.020 \mathrm{~m})$ is much bigger than $\Delta y(0.003 \mathrm{~m})$ and $\Delta z(0.005 m)$. One equation model may improve the LES simulation when the grid is relatively coarse.

The one equation subgrid-scale model with backscatter energy has also been tested in the flat plate wake. However, the results have not improved remarkably over that of without backscatter. The reason might be that the ratio of the subgrid scale TKE involved in the backscatterring was not appropriate.

\subsection{Summary}

The RFG and LES techniques have been applied to compute the wake of a flat plate. Unlike the traditional approach, the flat plate is not included in the computational domain. Instead, the simulation starts from a plane behind the flat plate. The instantaneous flow field at the inflow boundary is numerically generated using the RFG technique that provides the instantaneous velocity field at the inlet boundary in conjunction with the prescribed mean flow 
field obtained from experiments. This approach enables LES of high-Reynolds number flows with complicated geometries, such as those seen in ship wakes.

The flow information at the inflow boundary has been reproduced very well. The simulation results have been compared with the experiments. Good agreement has been obtained thus validating the LES procedure used in the remainder of this study.

Issues relevant to SGS models and grid resolutions have been discussed. With finer grids being employed, the resolution of turbulence scales hence prediction of turbulence statistics have been improved. 


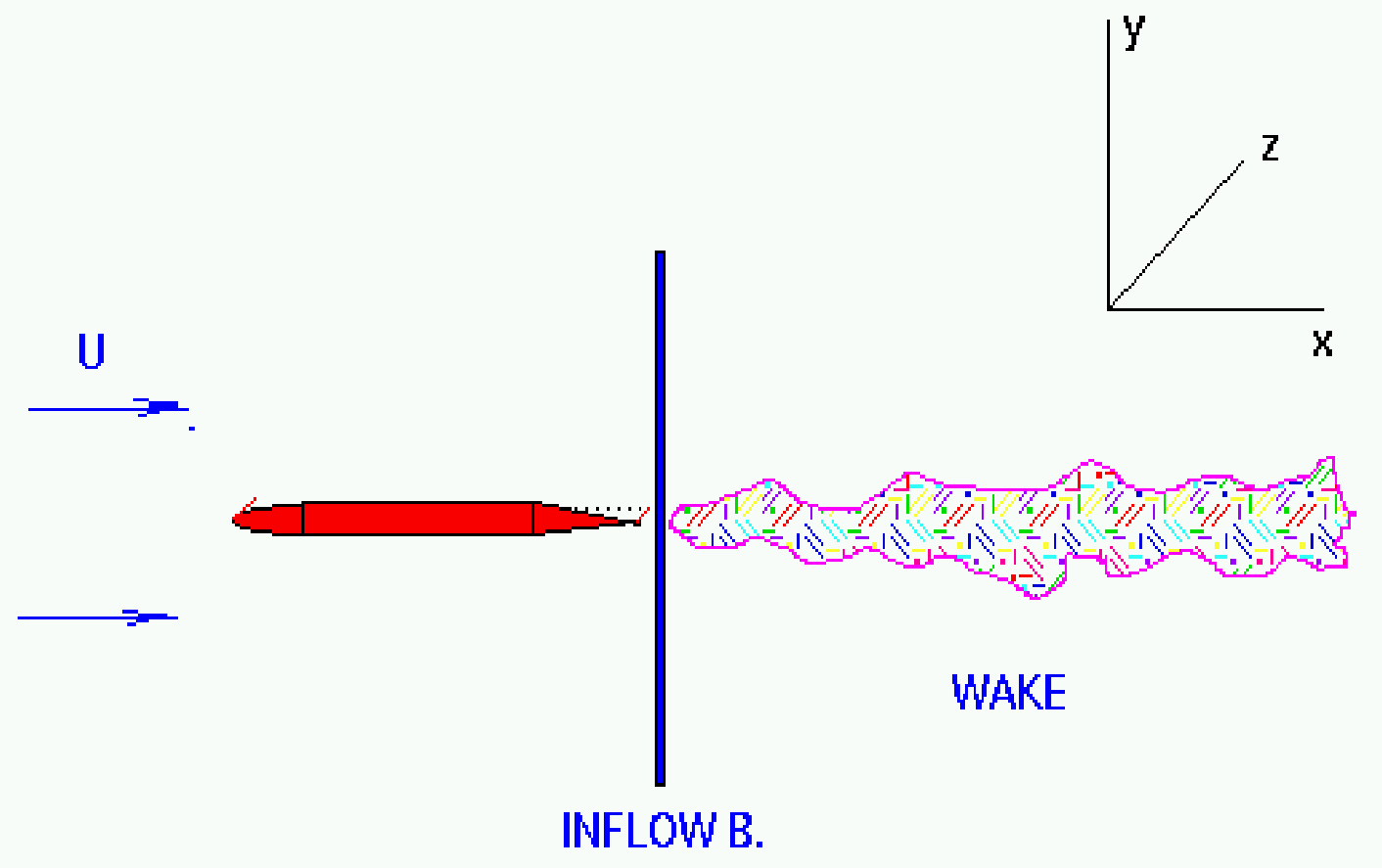

Figure 6.1: The schematic of the flat plate wake 

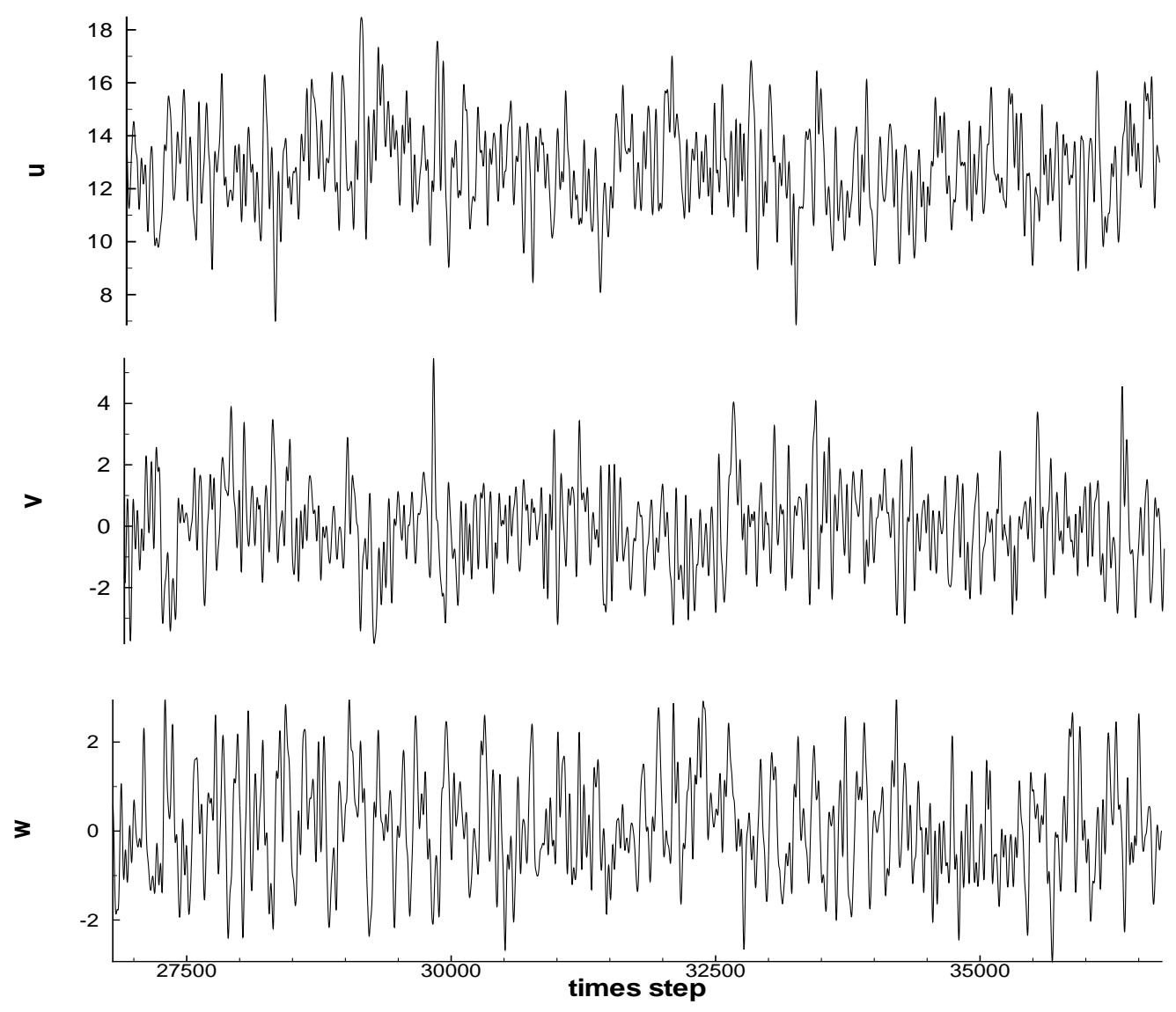

Figure 6.2: The velocities at the inflow boundary. Grid of $82 \times 50 \times 50$ are used along this chapter unless otherwise stated 


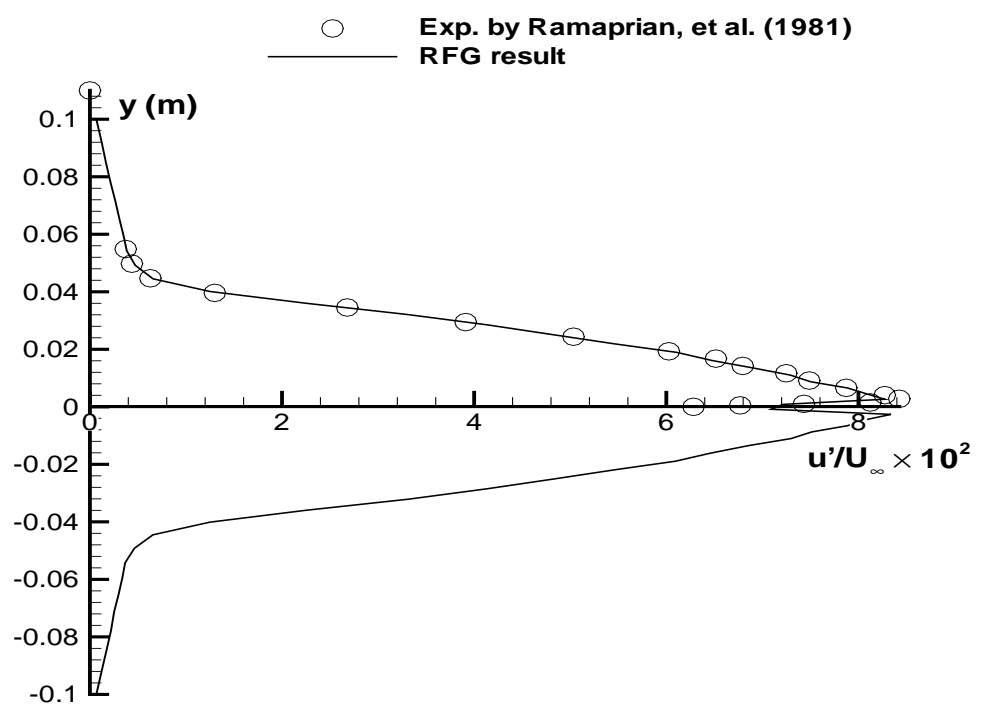

Figure 6.3: Turbulence intensities at the inflow boundary, $u_{r m s}$.

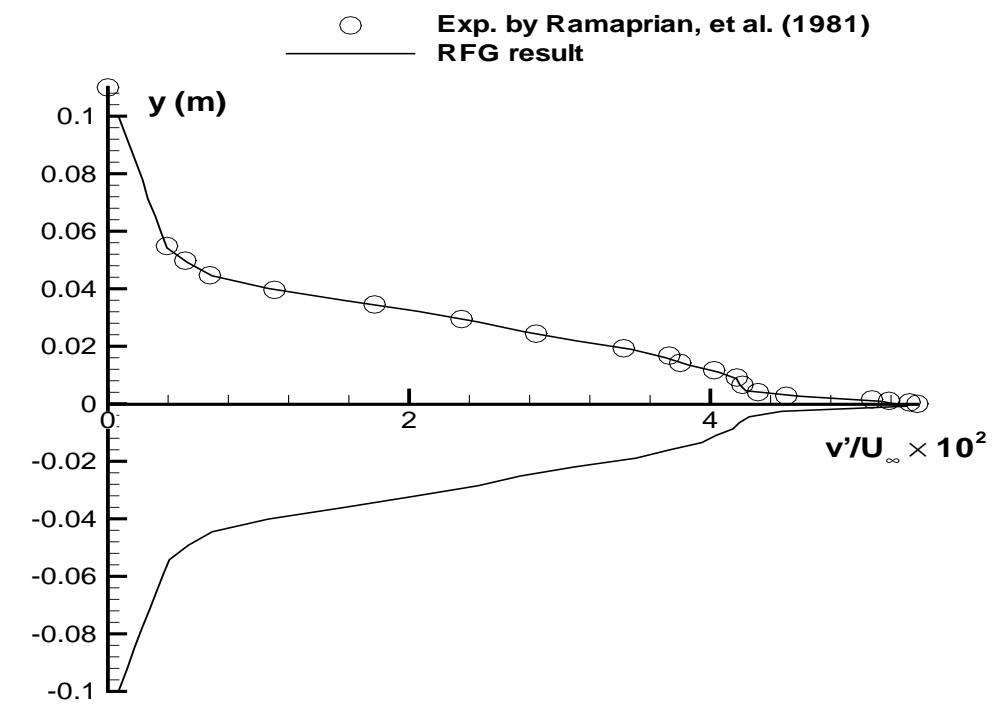

Figure 6.4: Turbulence intensities at the inflow boundary, $v_{r m s}$. 


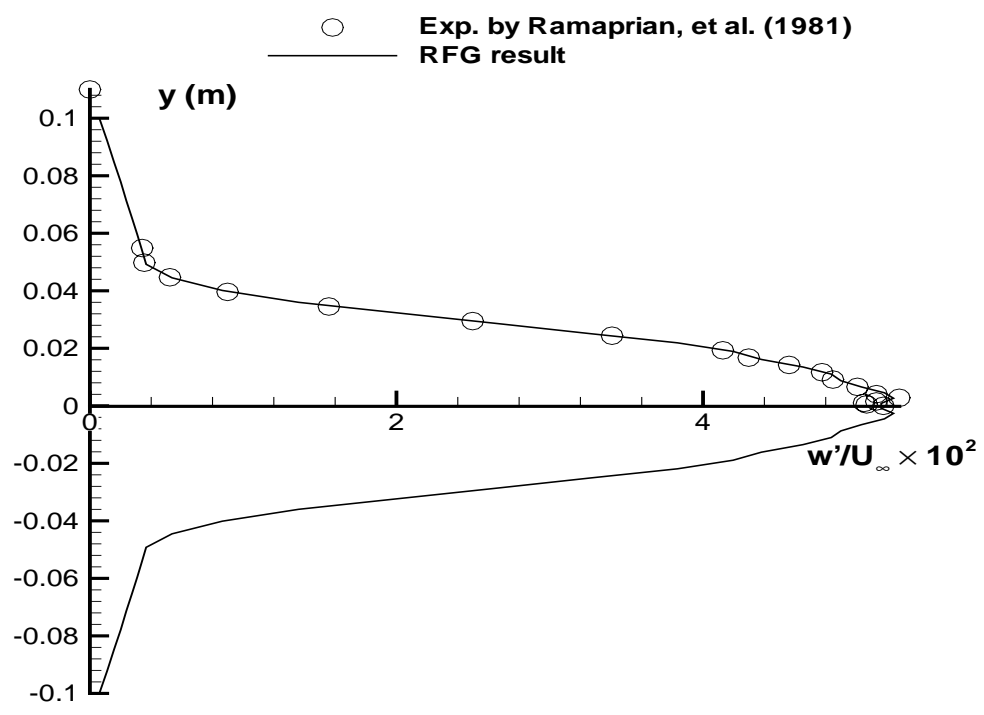

Figure 6.5: Turbulence intensities at the inflow boundary, $w_{r m s}$.

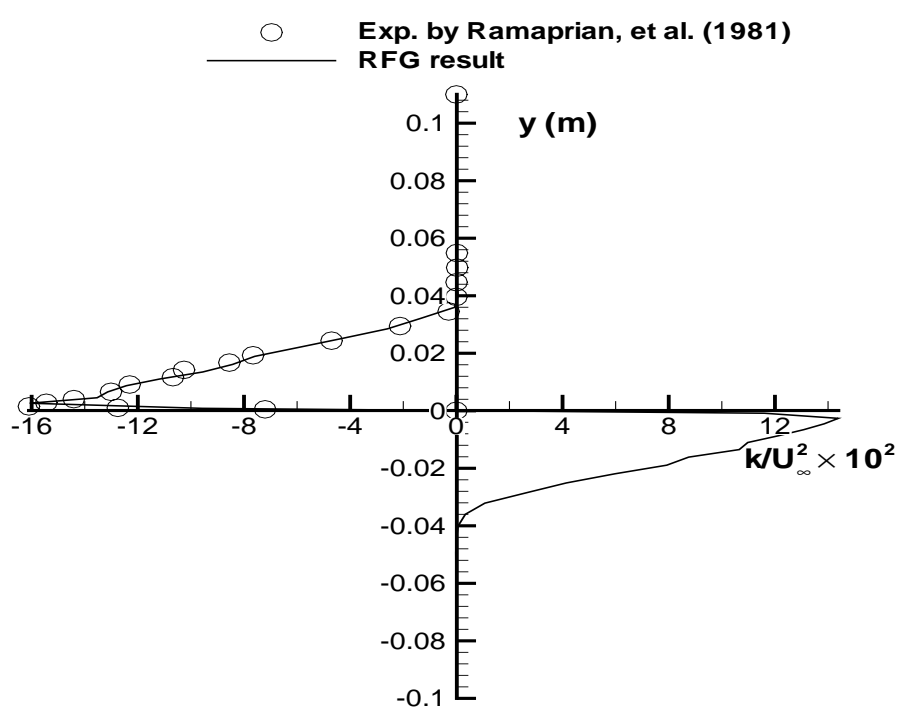

Figure 6.6: Turbulence intensities at the inflow boundary, $u v_{r m s}$. 

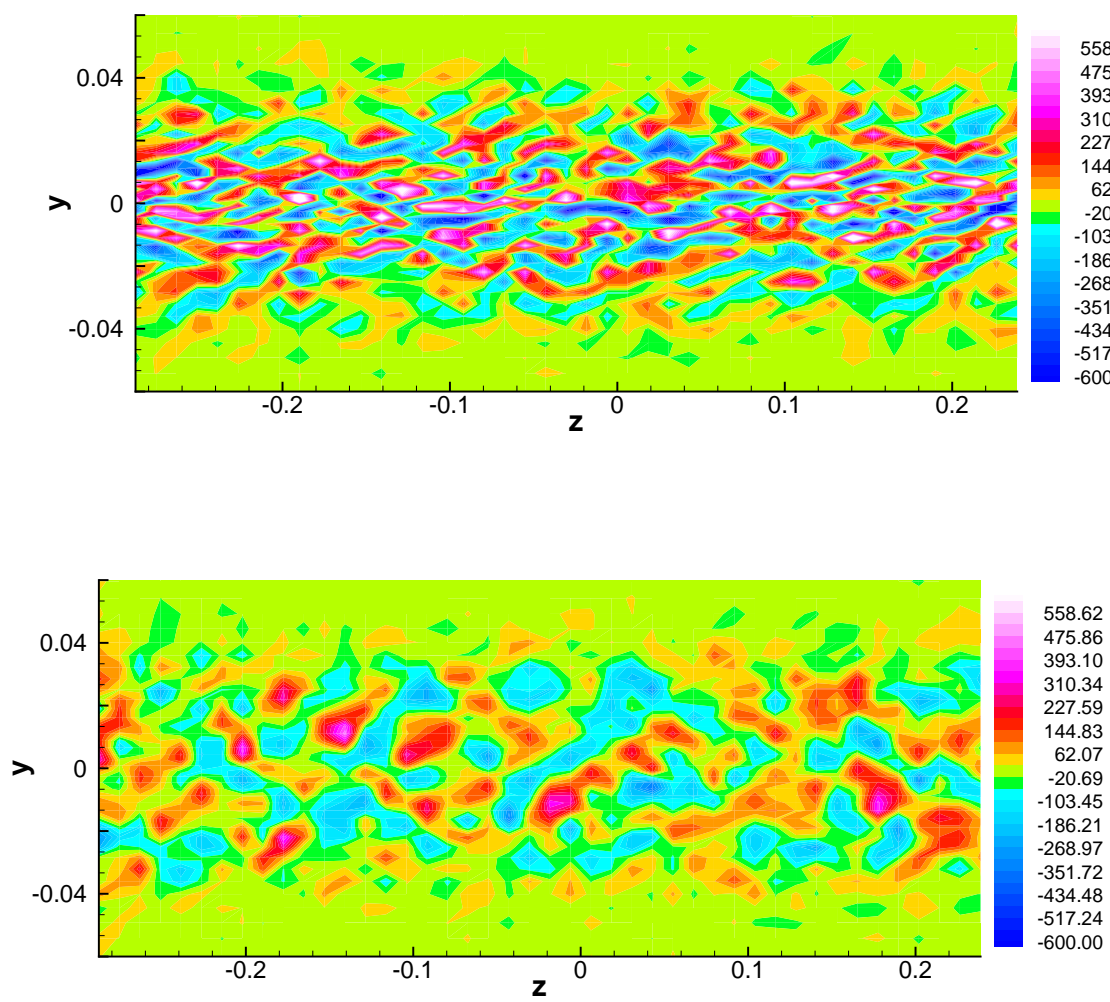

$x=0.2$

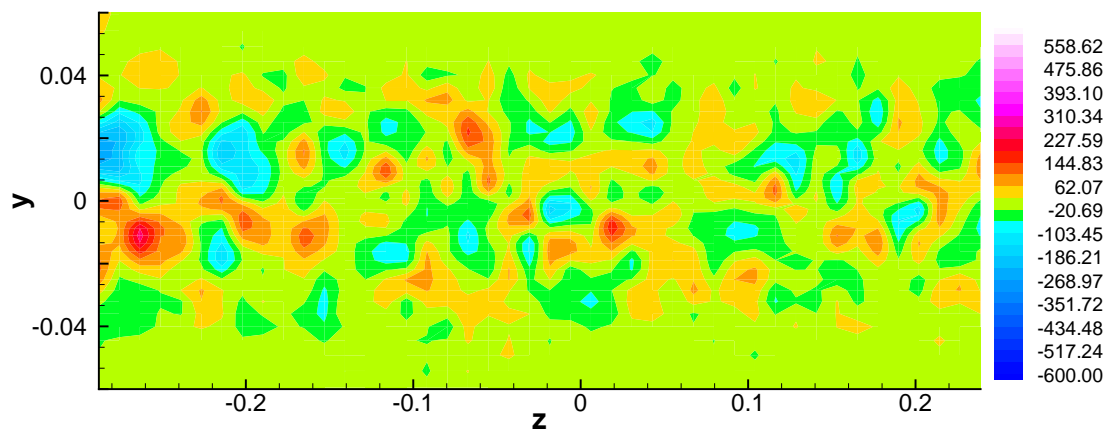

$x=0.4$

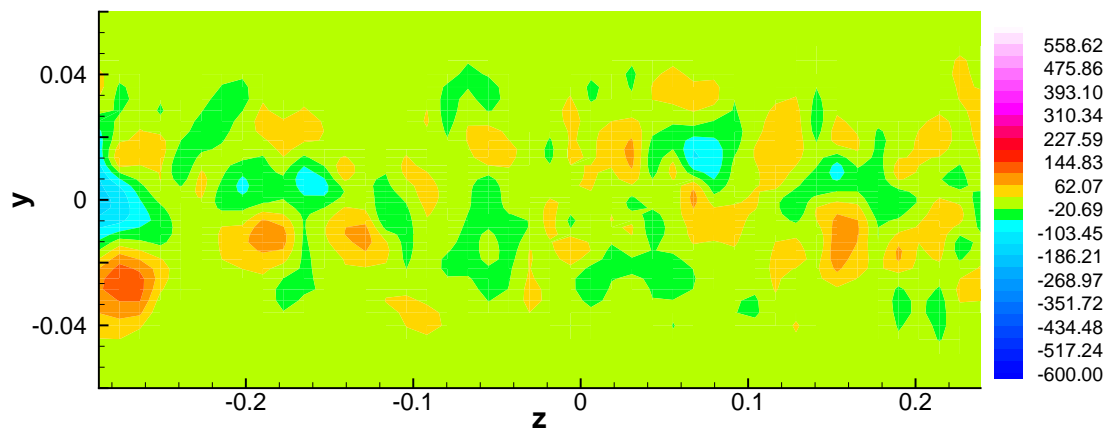

Figure 6.7: Typical streamwise vorticities $(\omega)$

$$
x=0.6
$$



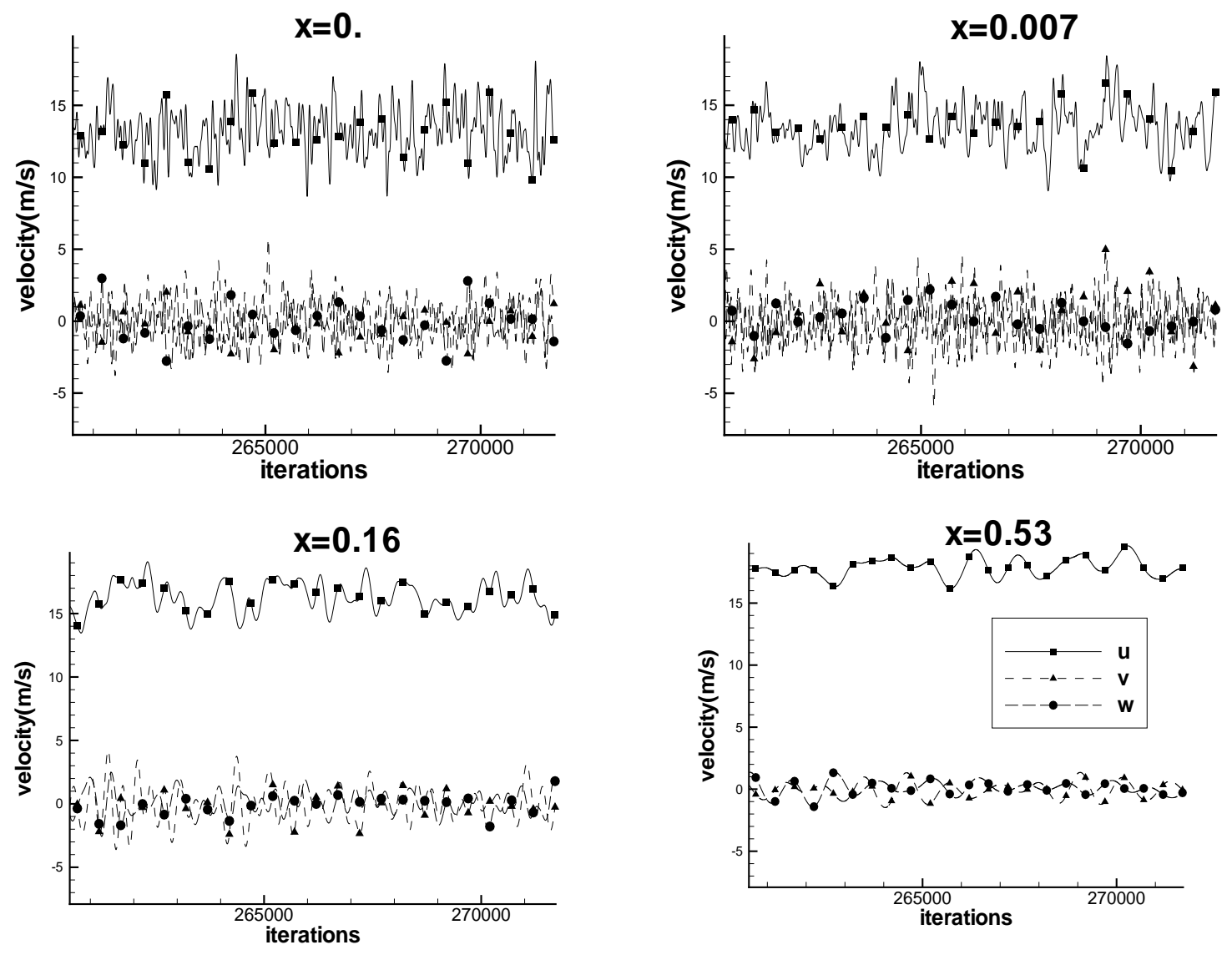

Figure 6.8: Temporal history of the instantaneous velocity components at different points in the flat plate wake $(y=0, z=0$ (center line) 


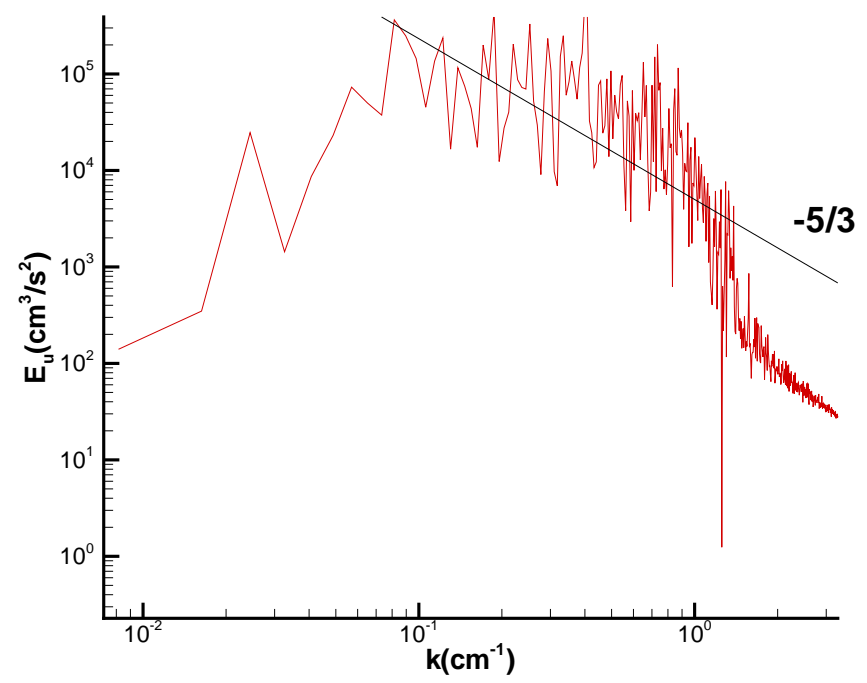

Figure 6.9: Energy spectrum at the same point as $x=0$ in Fig.6.8

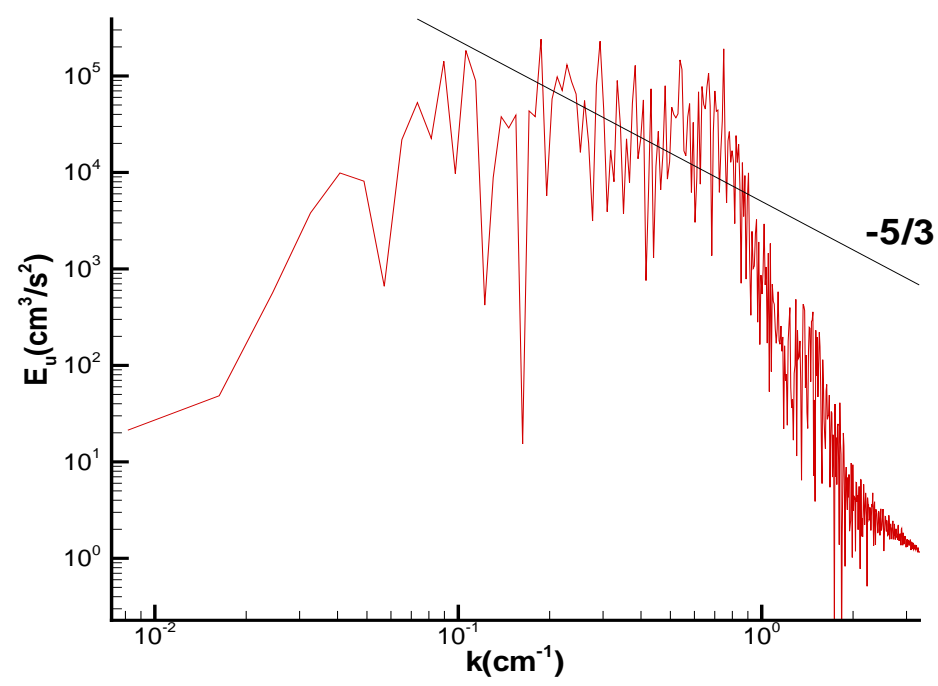

Figure 6.10: Energy spectrum at the same point as $x=0.16$ in Fig.6.8 


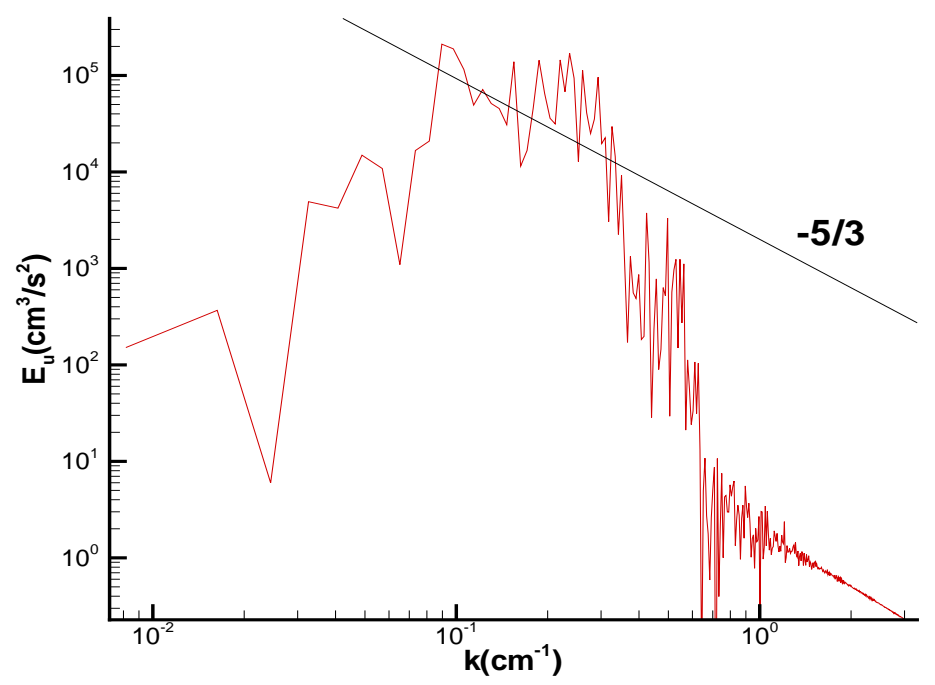

Figure 6.11: Energy spectrum at the same point as $\mathrm{x}=0.53$ in Fig.6.8 


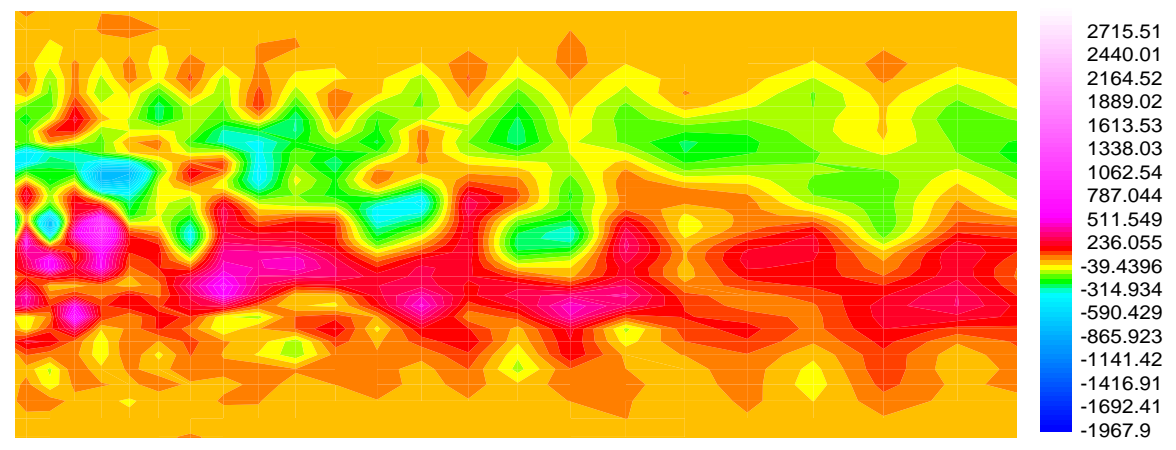

(a) Typical spanwise vorticity contours at one $x-y$ plane in the wake of the flat plate

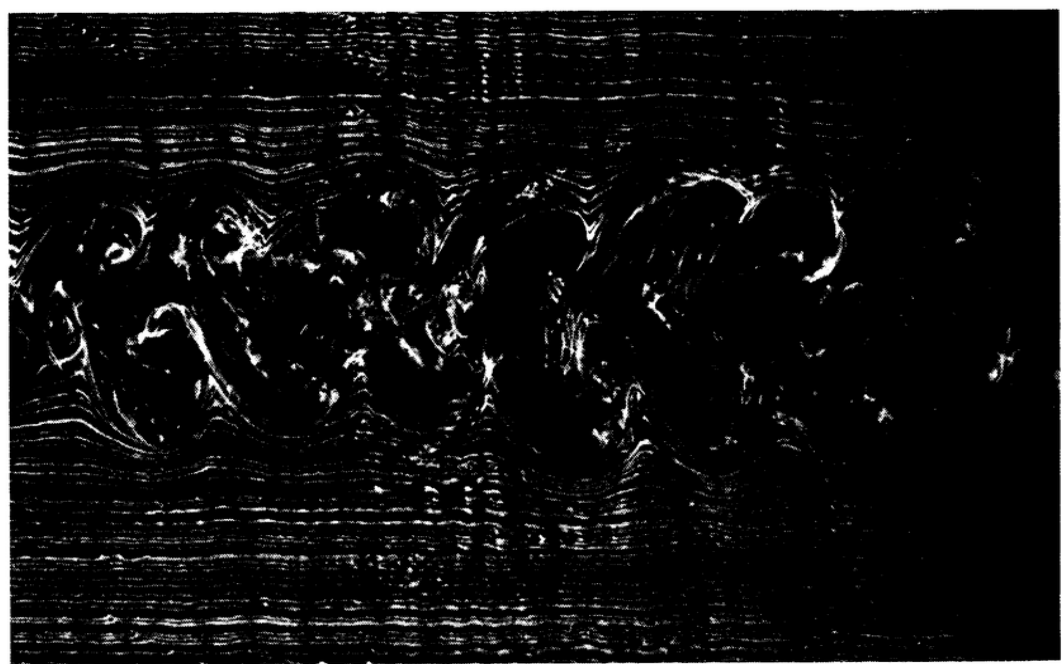

$\bar{x} \approx 400$

$\ddot{x} \approx 600$

(b) A photograph showing the large coherent structures in the flat plate wake (Wygnanski et al., 1986)

Figure 6.12: Comparison of the turbulence structures between LES and experiments 


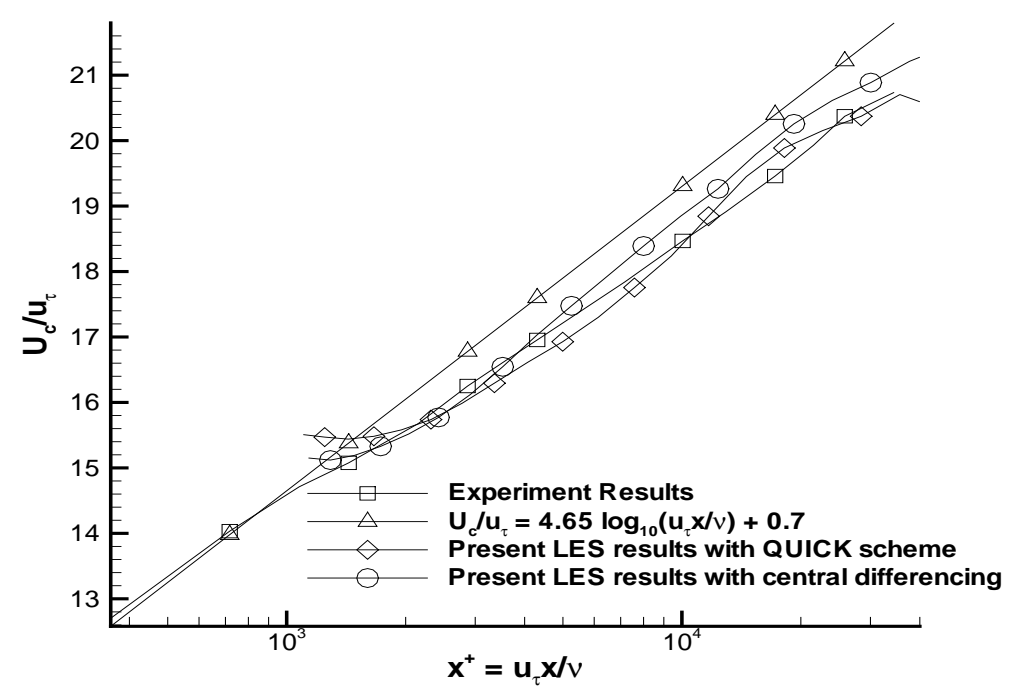

Figure 6.13: Centerline velocity in the wake of flat plate wake.

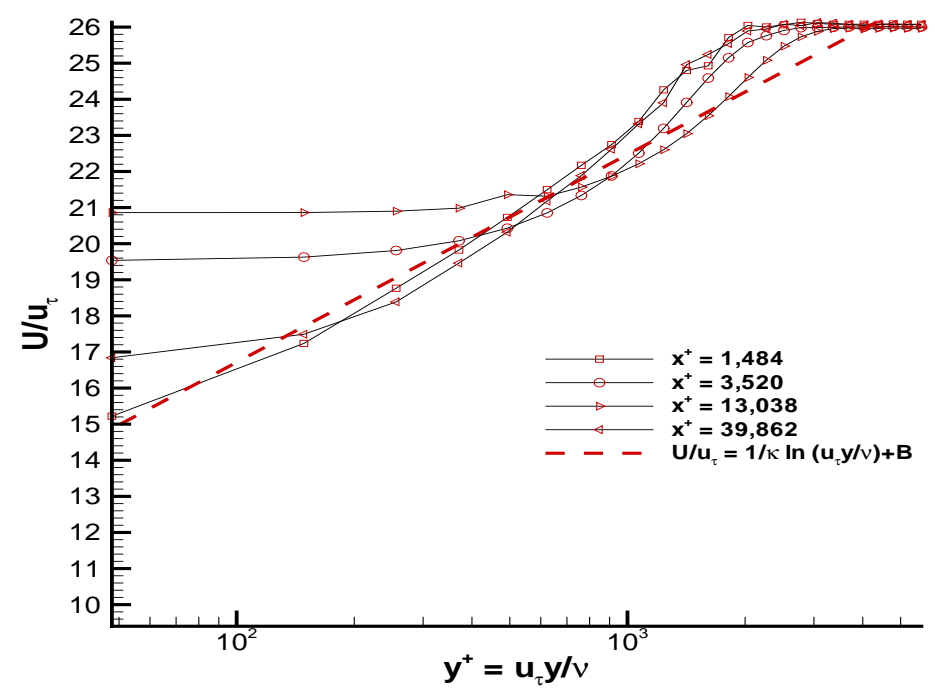

Figure 6.14: U-component mean-velocity profile in the wake of the flat plate 


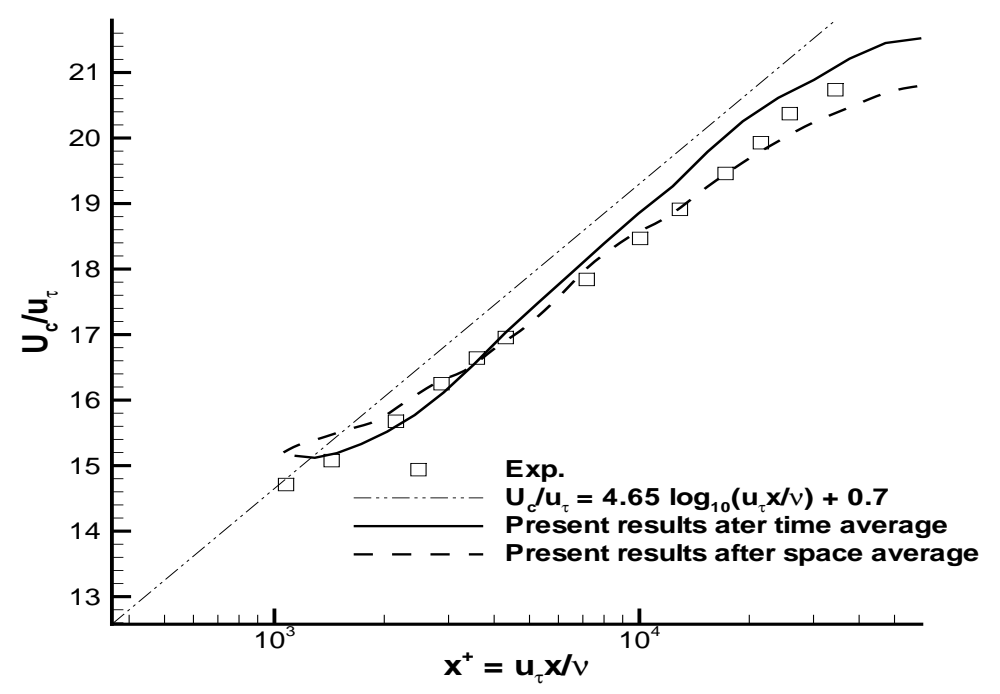

Figure 6.15: Centerline velocity in the wake of flat plate wake (for testing ergodicity).

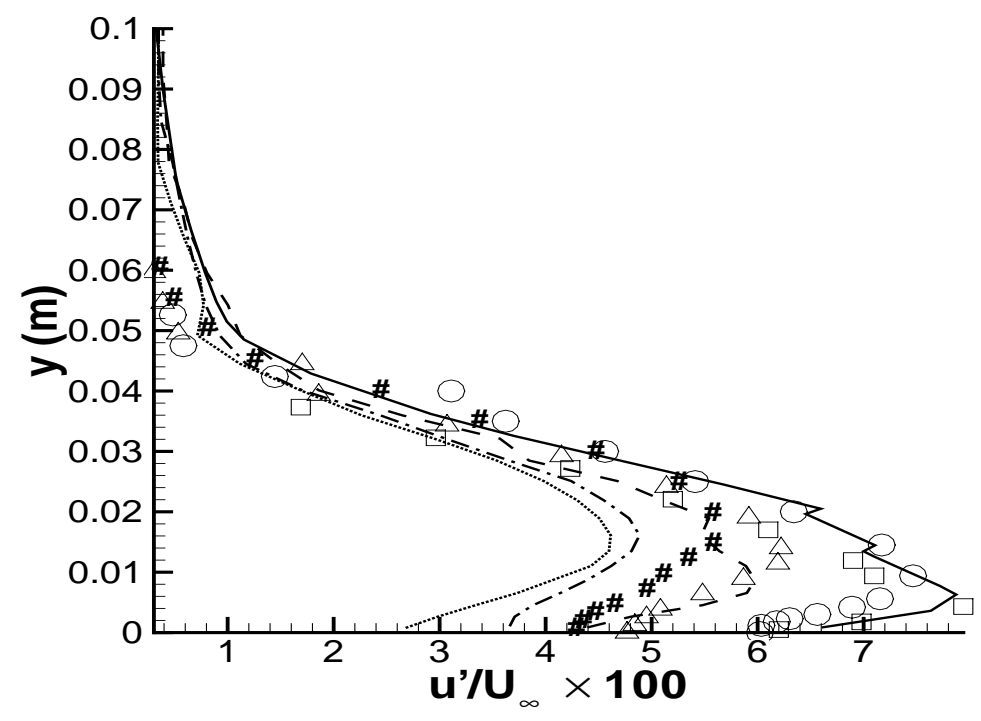

Figure 6.16: Comparison between simulation and measured turbulence intensity $\left(u_{r m s}\right)$. Present simulation:,$- x=31.75 \mathrm{~mm}$; - - - , $x=158.75 \mathrm{~mm}$; - - - -,$x=$ $361.95 \mathrm{~mm}$; $\cdots, x=590.55 \mathrm{~mm}$. Experiment results (Ramaprian, et al. 1981): $x=31.75 \mathrm{~mm}$; o, $x=158.75 \mathrm{~mm} ; \Delta, x=361.95 \mathrm{~mm}$; \#, $x=590.55 \mathrm{~mm}$; 


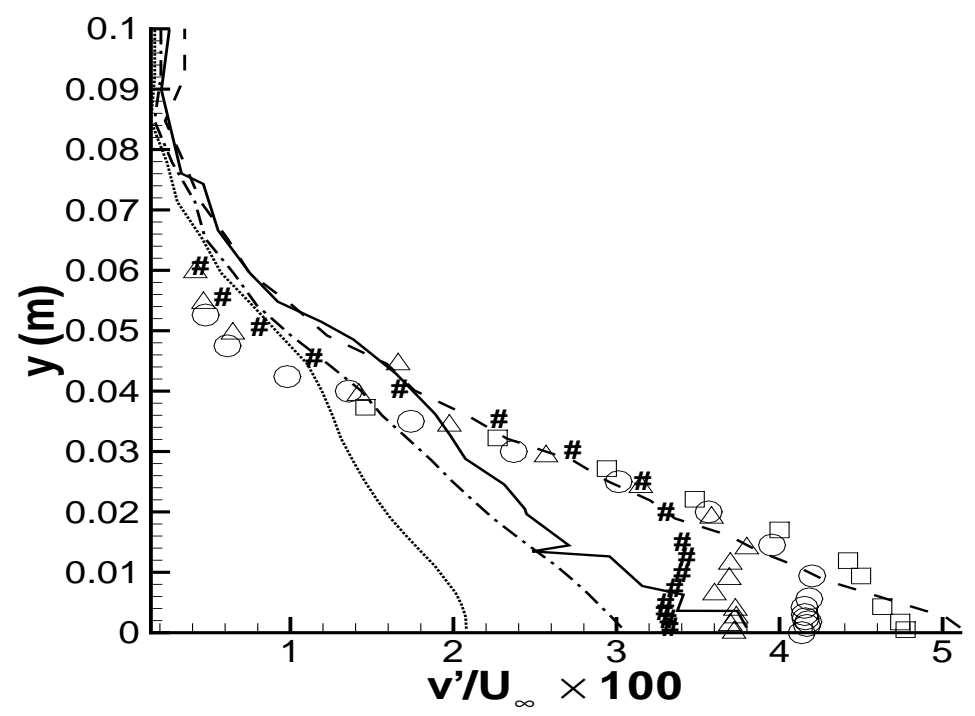

Figure 6.17: Comparison between simulation and measured turbulence intensity $\left(v_{r m s}\right)$. Present simulation:,$- x=31.75 \mathrm{~mm}$;,$--- x=158.75 \mathrm{~mm}$; $-\cdot-\cdot-, x=$ $361.95 \mathrm{~mm}$; $\cdots, x=590.55 \mathrm{~mm}$. Experiment results (Ramaprian, et al. 1981): $x=31.75 \mathrm{~mm}$; o, $x=158.75 \mathrm{~mm} ; \Delta, x=361.95 \mathrm{~mm}$; \#, $x=590.55 \mathrm{~mm}$; 


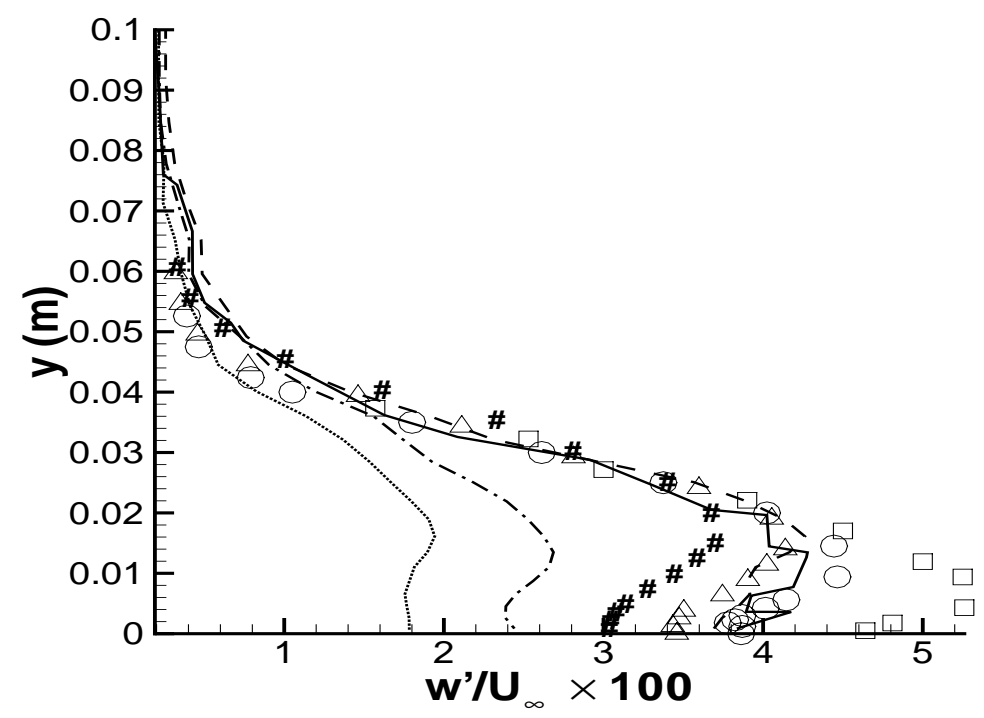

Figure 6.18: Comparison between simulation and measured turbulence intensity $\left(w_{r m s}\right)$. Present simulation:,$- x=31.75 \mathrm{~mm}$;,$--- x=158.75 \mathrm{~mm}$; $-\cdot-\cdot-, x=$ $361.95 \mathrm{~mm}$; $\cdots, x=590.55 \mathrm{~mm}$. Experiment results (Ramaprian, et al. 1981): $x=31.75 \mathrm{~mm}$; o, $x=158.75 \mathrm{~mm} ; \Delta, x=361.95 \mathrm{~mm}$; \#, $x=590.55 \mathrm{~mm}$; 


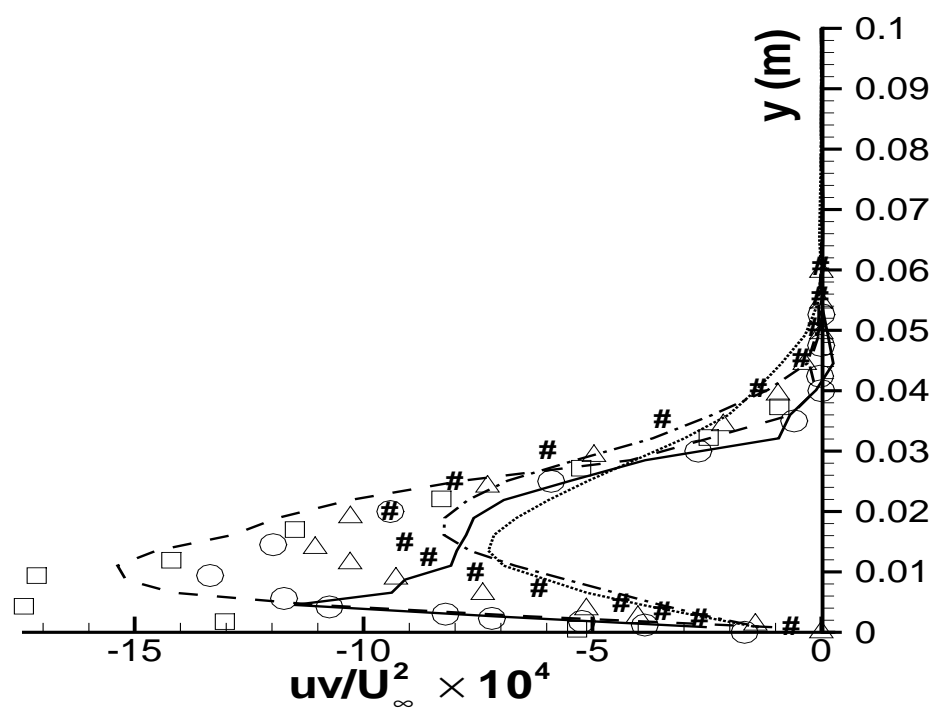

Figure 6.19: Comparison between simulation and measured shear stresses Present simulation:,$- x=31.75 \mathrm{~mm}$; - - - , $x=158.75 \mathrm{~mm}$; - - - -,$x=361.95 \mathrm{~mm}$; $\cdots, x=590.55 \mathrm{~mm}$. Experiment results (Ramaprian, et al. 1981): $\square, x=31.75 \mathrm{~mm}$; o, $x=158.75 \mathrm{~mm}$; $\Delta, x=361.95 \mathrm{~mm}$; \#, $x=590.55 \mathrm{~mm}$;

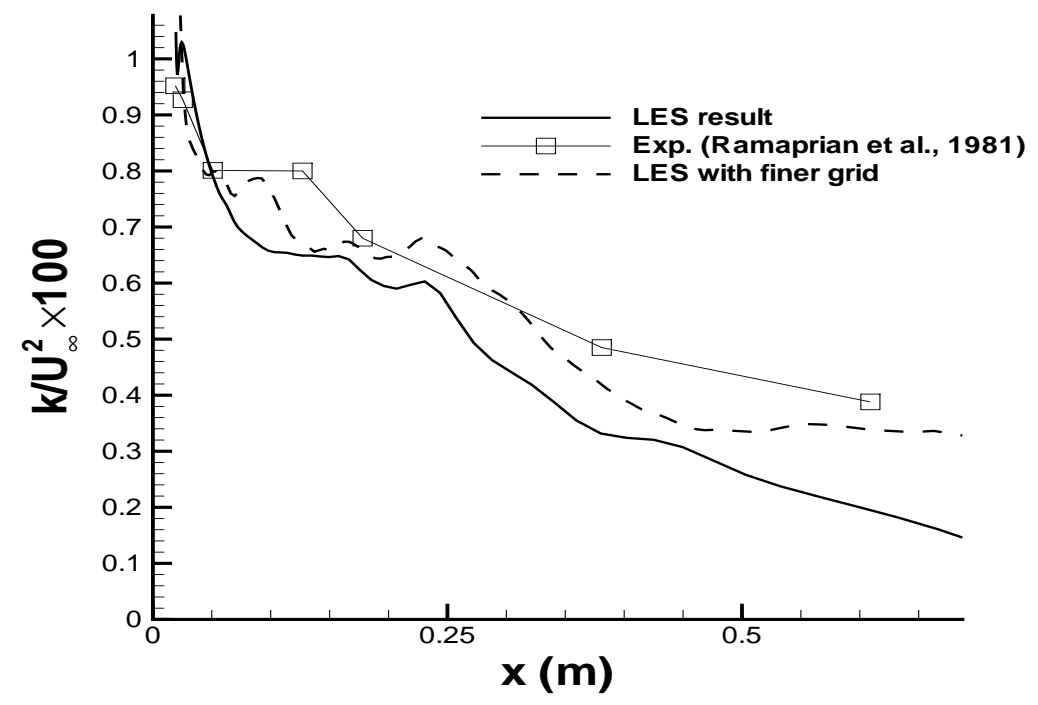

Figure 6.20: Turbulent kinetic energy profile along the center line in the wake of a flat plate 


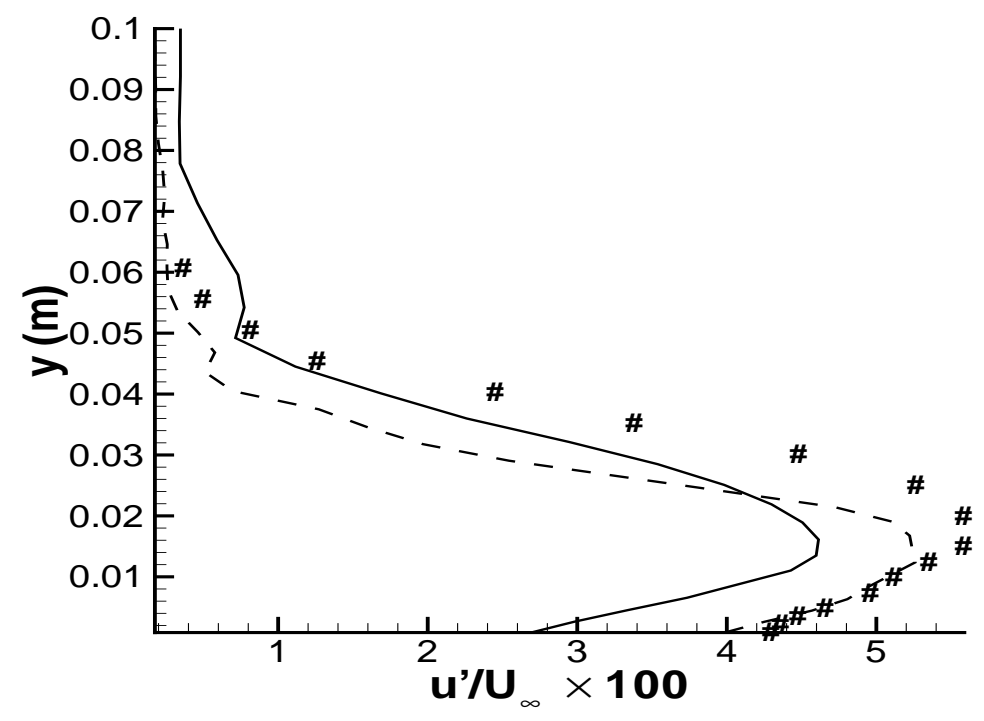

Figure 6.21: Turbulence intensity $\left(u_{r m s}\right)$ with finer grid at $x=590.55 \mathrm{~mm}$. - coarser grid solution; - - - , finer grid solution; \#, Exp. results (Ramaprian, et al. 1981) at $x=590.55 \mathrm{~mm}$;

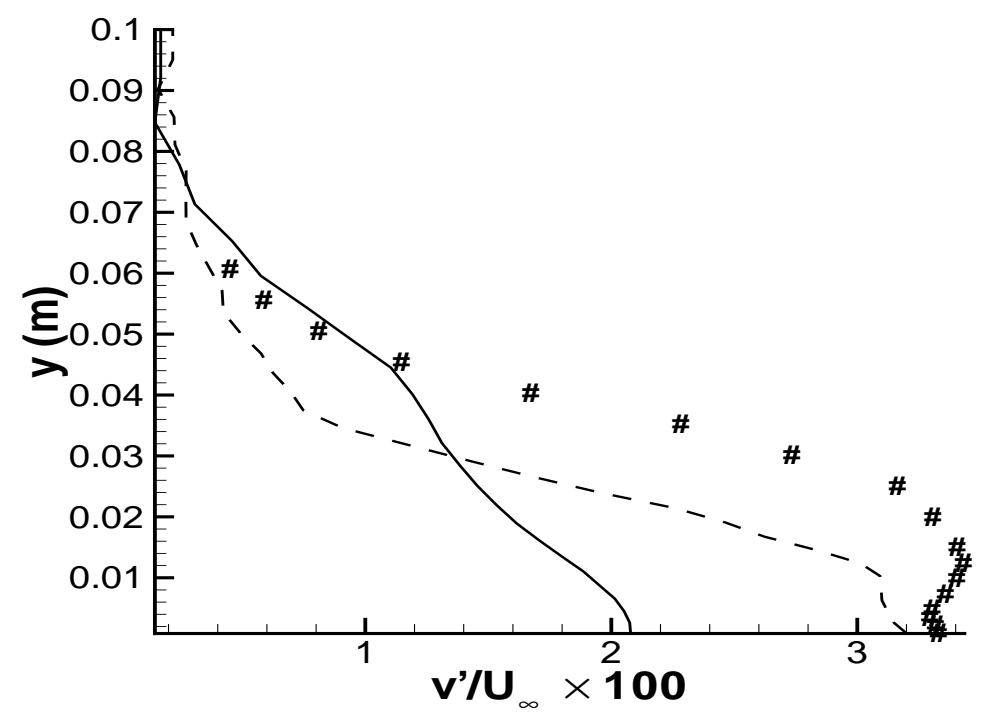

Figure 6.22: Turbulence intensity $\left(v_{r m s}\right)$ with finer grid at $x=590.55 \mathrm{~mm} . \longrightarrow$, coarser grid solution; - - - , finer grid solution; \#, Exp. results (Ramaprian, et al. 1981) at $x=590.55 \mathrm{~mm}$; 


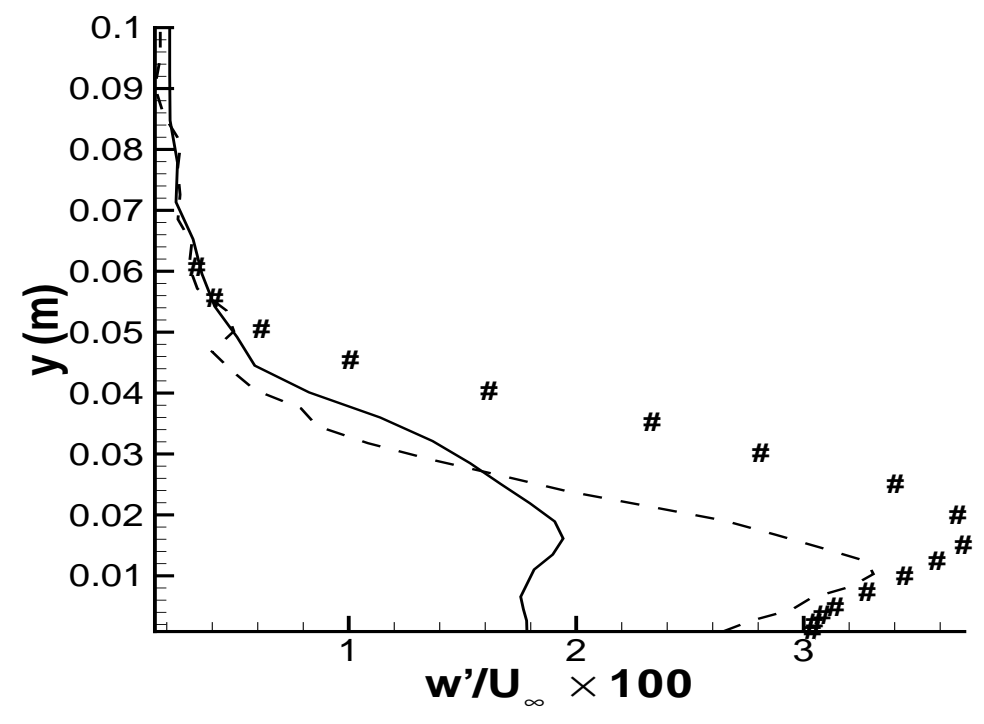

Figure 6.23: Turbulence intensity $\left(w_{r m s}\right)$ with finer grid at $x=590.55 \mathrm{~mm}$. — grid solution; - - - , finer grid solution; \#, Exp. results (Ramaprian, et al. 1981) at $x=590.55 \mathrm{~mm}$;

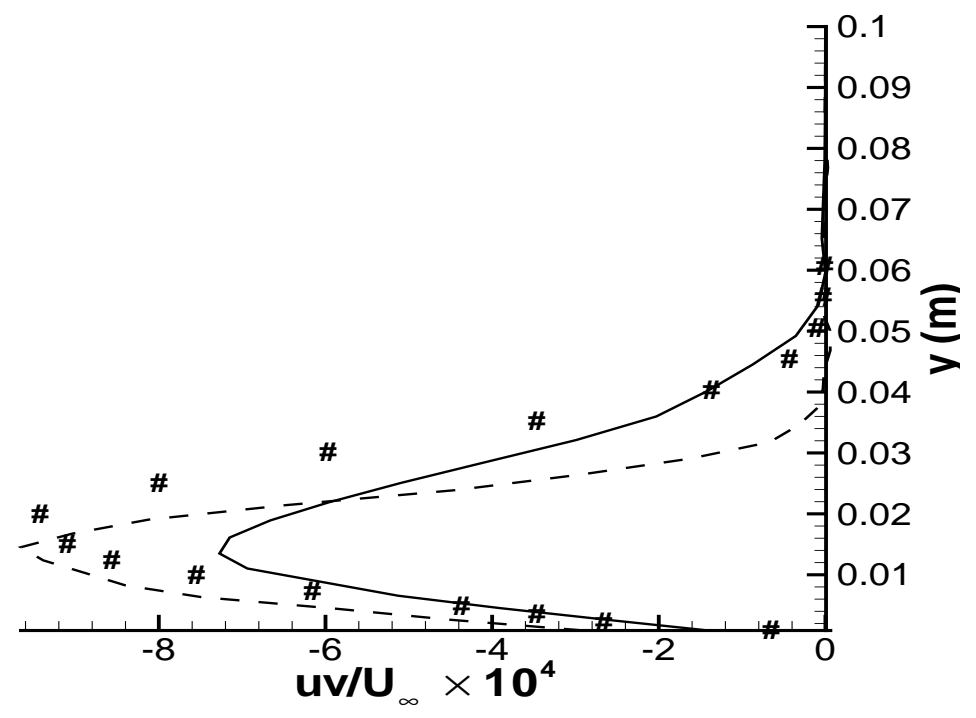

Figure 6.24: Turbulence intensity $(\overline{u v})$ with finer grid at $x=590.55 \mathrm{~mm}$. - , coarser grid solution; - - - , finer grid solution; \#, Exp. results (Ramaprian, et al. 1981) at $x=590.55 \mathrm{~mm}$; 


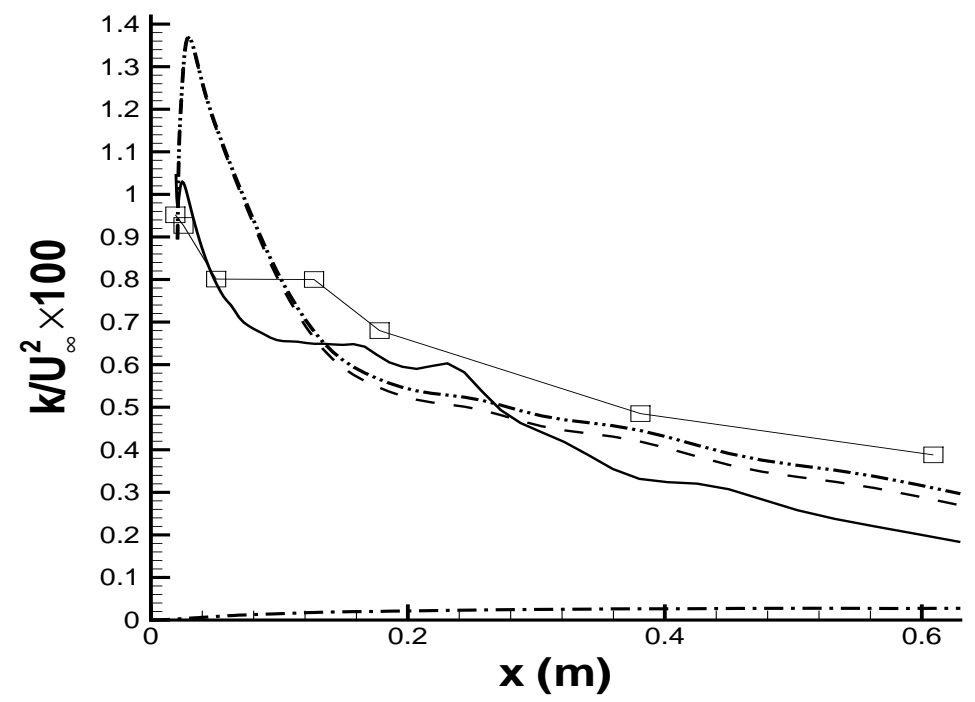

Figure 6.25: Turbulent kinetic energy profile along the center line in the wake of a flat plate with one equation model. $\square$ Experiment results (Ramaprian, et al. 1981); $\square$, Smagorinsky model; ----- , one equation model; $-\cdot-\cdot-$, subgrid-scale $k ;-\cdot \cdot-\cdot \cdot-\cdot--$, the total calculated TKE.

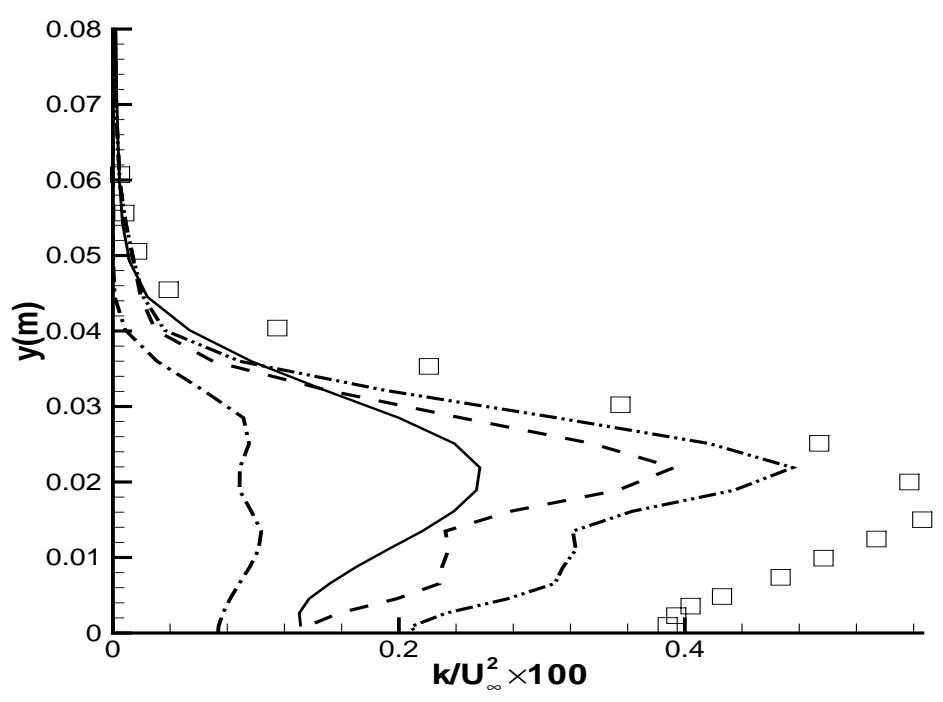

Figure 6.26: Turbulent kinetic energy profile along y direction at $x=590.55 \mathrm{~mm}$ with one equation model. $\square$ Experiment results (Ramaprian, et al. 1981); — $\_$, Smagorinsky model; ---- , one equation model; $-\cdot-\cdot-$, subgrid-scale $k ;-\cdots-\cdots-\cdots-$, the total calculated TKE. 


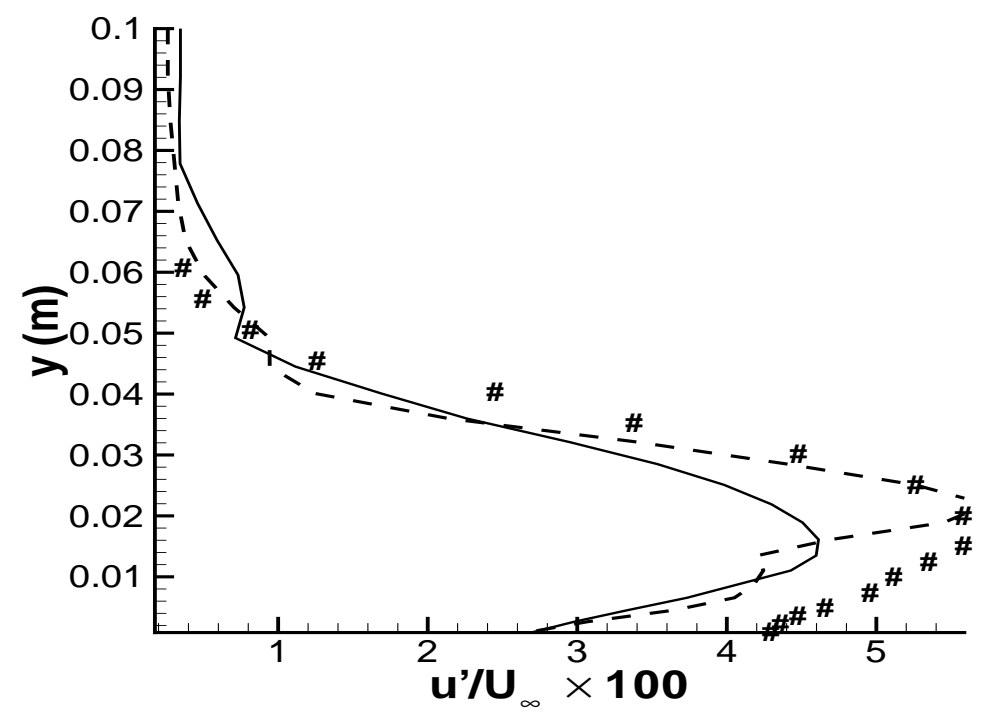

Figure 6.27: Turbulence intensity $\left(u_{r m s}\right)$ with one equation at $x=590.55 \mathrm{~mm}$. coarser grid solution; - - - , finer grid solution; \#, Exp. results (Ramaprian, et al. 1981) at $x=590.55 \mathrm{~mm}$;

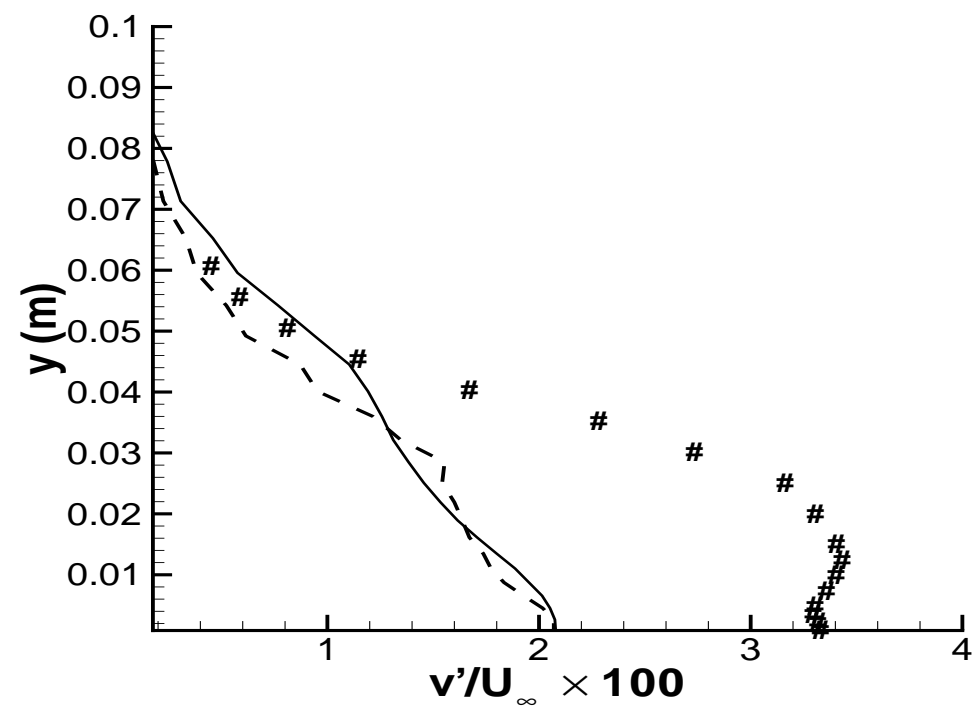

Figure 6.28: Turbulence intensity $\left(v_{r m s}\right)$ with one equation at $x=590.55 \mathrm{~mm}$. coarser grid solution; - - - , finer grid solution; \#, Exp. results (Ramaprian, et al. 1981) at $x=590.55 \mathrm{~mm}$; 


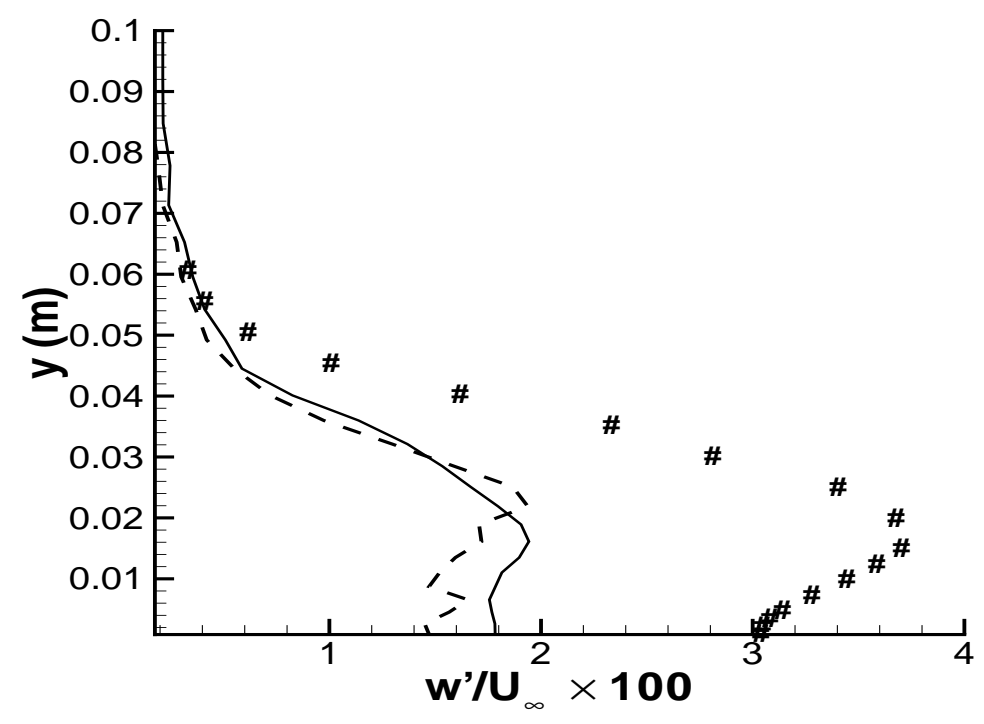

Figure 6.29: Turbulence intensity $\left(w_{r m s}\right)$ with one equation at $x=590.55 \mathrm{~mm}$. coarser grid solution; - - - , finer grid solution; \#, Exp. results (Ramaprian, et al. 1981) at $x=590.55 \mathrm{~mm}$;

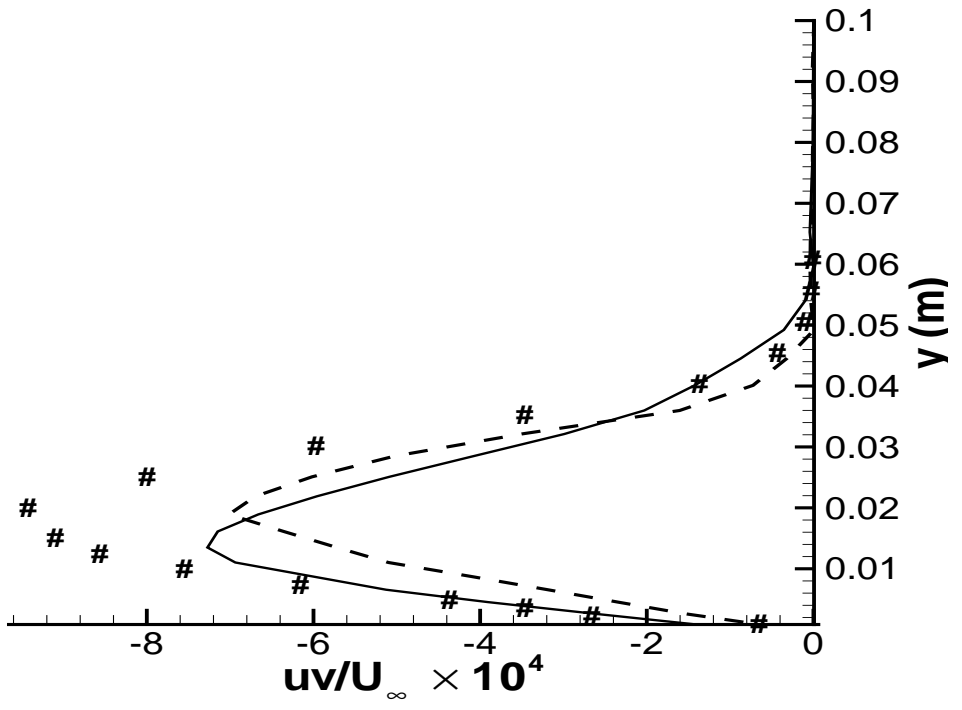

Figure 6.30: Turbulence intensity ( $u v$ ) with one equation at $x=590.55 \mathrm{~mm}$. coarser grid solution; - - - , finer grid solution; \#, Exp. results (Ramaprian, et al. 1981) at $x=590.55 \mathrm{~mm}$; 


\section{Chapter 7}

\section{Application to Ship Wake}

The subject of this Chapter is the application of the numerical schemes and SGS models described in previous chapters to the calculation of turbulent ship wake flows. Starting from the review of the current research status of ship wake hydrodynamics, this Chapter leads to the summary of the strategies described in previous chapters. Several cases have been simulated and results are analyzed and assessed.

\subsection{Introduction}

Ship hydrodynamics has been the subject of numerous studies in the past. Both experimental and numerical achievements have been made. Since real ships can not be fitted into tow tanks, it is common practice to base the research on small-scale models of real ships. Experimentally, in the 60's or 70's of the last century, most of the experiments concerned the ship surface drag force, or propeller influence. Recently, experiments are more focused on the investigation of micro dynamics of flow around ships, such as wave dynamics, turbulence parameters, bubble dynamics, etc. Although experiments can provide useful results, there are many limitations encountered in practice. First, it takes a very long time to design, set, and perform the experiment. Secondly, it is very expensive. Thirdly, it can not provide very detailed information due to the limited space to arrange the measuring devices or detectors. Benefited from the rapidly developing capabilities of computers, the modern 
research in computational hydrodynamics has replaced the more elegant, but less general, analytical methods (Larsson et al., 1998). Since ship wake flows are the main subject of this study a separate but concise review is presented next.

\subsubsection{Experiments on turbulent ship wake flows}

In a earlier study of ship wake flows, Naudascher (1965) found that the wake width has a power law behavior. This was supported by Milgram et al. (1993). By carrying out field measurements for ship wakes, they found that the wake width has a power law behavior of the type $x^{1 / 5}$ where $x$ is a distance from the ship stern. Reed et al. (1990) summarized the hydrodynamics research of ship wake flows both in experimental and numerical areas and pointed out the hydrodynamic wake schematic shown in Fig. 7.1. Benilov et al. (2000) found that even in the far wake the ship wake turbulence is still well detectable and the Kolmogorov range can be identified. Hoekstra (1991) gave relatively comprehensive tests of turbulence parameters in which the wakes of different ship models were studied. The vortices generated by the ship body, named bilge vortices, were analyzed, and it was found that these vortices can be avoided by hull form adjustment but this was found to be impractical. Stern et al. (2000) present some results for CFD validation, but the experiments providing turbulence intensities are still underway, and hopefully some results from them will be available in the coming years. For this study it was not possible to find the necessary experimental information to initialize an LES study, or to build the inflow boundary condition including the turbulence features induced by the ship hull. Thus the current simulation is based on RANS results.

\subsubsection{Computations of turbulent ship wake flows}

Most of the computational fluid dynamics efforts applied to flow past ships are based on Reynolds-Averaged Navier-Stokes (RANS) equations utilizing various turbulence models 
(Sotiropoulos and Patel, 1995; Paterson et al., 1996; Ratcliffe, 1998). The commonly used models include $k-\epsilon, k-\omega$ and algebraic stress models. RANS is often quite adequate for mean flow predictions, but provides only limited information about turbulence characteristics and almost no details on large-scale unsteady structures of the flow field. The LES technique, on the other hand, was designed to capture the unsteady behavior of at least the large coherent turbulent eddies. There is hardly any study in the literature where LES has been applied to flow around ship-hulls including the wake. The main reason obviously lies in the fact that large computer resources are required. In LES, the energy containing eddies of the flow are computed explicitly, while only the more universal (isotropic) small eddies are modeled. Thus very fine grids have to be applied in order to resolve the boundary layer near the wall where the turbulence length scales tend to zero as the wall is approached. However, in some applications, like bubble dynamics modeling, it is still necessary to resolve coherent flow structures - large turbulent vortices and eddies. In RANS simulations, especially those using two-equation models such as $k-\epsilon$ model, these unsteady flow features are usually smeared out.

Since it can be computationally prohibitive to include both the ship hull and the wake in LES, it would be desirable for the purposes of pure wake simulations to start the computations somewhere in the near wake excluding the ship hull. However, this technique, also known as the Initial Data Plane (IDP) approach (Hyman, 1998; Paterson et al., 1996; Dommermuth et al., 1996) can introduce considerable errors (Hyman, 1998) - not surprisingly though, since the body generating the wake is not included in the non-steady LES simulations.

In principle it is possible to predict turbulence via the LES technique by starting from a quiescent flow or with the mean flow obtained from RANS. Unfortunately, it takes a very long time for a turbulent flow to develop spatially and temporally without any initial perturbation. This is especially true in the case of decaying turbulence in the absence of strong 
turbulence generating factors like walls. A reasonably accurate approach to this problem was used in modeling of boundary layer turbulence (Lund et al., 1998). It consisted of applying a separate flow solver with periodic boundary conditions to construct the inlet conditions for the LES/DNS solver. It provides fully turbulent inflow conditions consistent with the solution of the Navier-Stokes equation, which makes it particularly suitable for DNS. This method was used later by Wang and Moin (2000) to compute the inflow boundary for simulating a trailing-edge flow. However, its implementation may not be straightforward for the problems without well defined fully developed boundary/shear layers. For some engineering problems it may also be too expensive in terms of computer resources and programming effort.

Another numerical simulation with time dependent turbulent inflow boundaries was performed by Voke and Potamitis (1994) for a wake of a flat plate. In their simulation, the inflow boundary information was derived from a separate simulation, called the precursor simulation, in which a low Reynolds number turbulent boundary layer was simulated. At the inflow plane of the wake simulation the three velocity components were specified by reading one transverse plane of velocity data from the boundary layer simulations at each time step. The transverse plane was selected at a location where the flow was fully turbulent but with a safe distance from the outflow boundary of the precursor simulation. Two separate sets of velocity data of the boundary layer were needed to provide inflow conditions for the upper and lower halves of the wake simulation. To achieve this they used the data of the same precursor simulation of the boundary layer at well-separated times in it's history. This method is costly and only applicable to parabolic wake flows. In application to the ship wake flow it would require LES of the flow around the hull itself which is impractical.

To achieve a higher accuracy of ship-wake simulations and still remain within realistic constraints on computer resources, the whole problem of computing the flow past ships and their wakes is divided into two parts: steady-state RANS calculations around the ship hull, 
and non-steady LES of the wake. In this approach the LES starts from a plane in the near wake where the inflow conditions are retrieved from the RANS results. This is similar to the IDP approach used by Hyman (1995) and Dommermuth et al. (1993) where the data reflects the mean flow, mean turbulence quantities and the scalar field at this plane. In the present approach, a wider range of turbulent quantities at the inlet plane are allowed, which include length- and time-scales, and all nine components of the turbulent shear-stress tensor (only six components to be computed due to symmetry), thus providing anisotropic and inhomogeneous turbulent inlet conditions. The technique is based on a Random Flow Generation (RFG) algorithm (Celik et al., 1999; Smirnov et al., 2001b; Shi et al., 2000b), which is applied to generate the inflow turbulence on the basis of the information obtained from experiments or RANS calculations. The generated velocity fluctuations satisfy instantaneous continuity equation, and the turbulence statistics (Reynolds stresses) are prescribed a priori. Thus in some sense, although the LES starts at a plane behind the body, the influence of the body is implicitly included. The features of the generated flow-field such as continuity, anisotropy and inhomogeneity make the RFG method also well suited for setting the initial conditions for LES.

In the present simulations anisotropic, inhomogeneous, unsteady IDP conditions were applied. Thus the flow develops according to the dynamics prescribed by the Navier-Stokes equations without forcing and it is believed more reasonable in the near wake. This approach differs from that of Dommermuth et al. (1996) which is appropriate for the far wake field (Hyman, 2001)

To describe the dynamic behavior of the turbulence in the ship wake, in another study (Benilov et al., 2000) made the following assumptions:

1. The wake turbulent kinetic energy significantly exceeds the upper layer turbulence that reduces the turbulent wake problem to the turbulent region development in a non-turbulent liquid. 
2. The main source of turbulence is a moving ship which means that all interactions between the wake turbulence and environment do not contribute in the wake dynamics and allow the problem to be reduced to the shear-free turbulent model.

Through all this study, those assumptions were taken to simplify the problem which is to perform LES on the wake flow of the ship model DTMB 5415 (5512)

\subsection{Ship Model DTMB 5415}

The ship wake simulations in the present study are for the ship model DTMB 5512, a smaller ship model scaled from DTMB 5415 which are well known in the ship hydrodynamics community. Model 5415 is a towing tank model representing a modern naval combatant. It was constructed out of wood and measured $5.72 \mathrm{~m}(18.767 \mathrm{ft})$ between perpendiculars. Model 5512 measures $3.04 \mathrm{~m}$ (10.000 feet) between perpendiculars. It is an appended version of 5415, with shafts, struts, rudders and propellers. However in this study it has been simplified as a bare hull model. The geometry of this ship model is shown in Fig. 7.2. Note that this picture is not scaled, and it is only used to show the profile of the ship model.

\subsection{Designing of Cases Studied}

\subsubsection{Case 1: Ship wake with flat free-surface}

In order to avoid solving for the flow around the bodies, the computational domain starts from the inflow boundary (initial data plane) located immediately after the body in the wake where there is no flow-reversal. The pseudo-random flow field generated by the RFG method is added to the mean flow of RANS simulations to establish the boundary condition at the inlet plane. 
The whole ship flow including the ship wake can be sketched as in Fig.7.3. The computational domain starts from $x / L=1.05(x / L=1$. is the end of the ship model). At these planes the RFG method described in Chapter 4 is used in conjunction with the RANS calculations (Stern and Wilson, 2000).

At the inflow boundary all components of the velocity were specified as a function of time and space. At the outflow boundary both convective and Neumann (free gradient) boundary conditions were applied. A comparison (not shown here) indicated that there is not much difference between the results obtained by applying these two conditions. The reason is that, as indicated by Voke and Potamitis (1994), to make the convective type outflow boundary work properly the grids at the outflow range should be very fine. However, in the present study, the grids were much coarser because fine grids have to be used in the upstream regions to capture the initial development of the turbulence.

Symmetry conditions have been used in y direction and periodic boundary conditions have been used in the spanwise (z) direction. At the free surface a slip condition is allowed in $\mathrm{x}$ and $\mathrm{z}$ directions but the velocity component normal to the free surface is set to zero. As such the free surface is approximated as a moving flat plane.

The computational domain size is $1.5 \times 0.3 \times 0.6$ (given in non-dimensional units in ship length) in $\mathrm{x}, \mathrm{y}$ and $\mathrm{z}$-directions, respectively. The grid size is $162 \times 50 \times 66$. Nonuniform grid spacing, stretching smaller than 1.03, is used in both $\mathrm{x}$ and $\mathrm{y}$ directions.

The length scale and time scale used in RFG were selected as constant in this case. The length scale was 0.02 of the ship length, and the time scale was 0.001 , nondimensionized by free stream velocity and ship length. Those numbers were selected because the turbulence length scale is about $15 \%$ of the ship width.

The Smagorinsky SGS model, and second order central differencing scheme were used unless otherwise stated. 


\subsubsection{Case 2: Ship wake with sinusoidal free surface}

At this stage, the purpose is to test the capability of the code, which is able to solve the unsteady Navier Stokes equations in a boundary fitted curvilinear coordinate system. It is also of interest to explore the effect of the surface waves on the turbulent kinetic energy distribution.

A s a first approximation the free surface profile was prescribed by

$$
\begin{gathered}
d y=a \times \frac{1}{[(|x|+1)(|z|+1)]^{1 / 2}} \sin (2 \pi / \lambda x+\pi / 2), \\
\lambda=b \times[(|x|+1)(|z|+1)]^{1 / 2} .
\end{gathered}
$$

where $d y$ is the variation from flat surface; $a=0.025$ and $b=0.3$ are constants to define the amplitude of the "wave".

The computational domain is shown in Fig.7.4.

The boundary conditions as well as the grid number, time scale and length scale for RFG were the same as those used for the case with a flat free surface.

The Smagorinsky SGS model and second order central differencing scheme were used.

\subsubsection{Case 3: Simulations including part of the ship hull with wave free surface}

Up to this point, the computational domains employed to study of wake flows started from somewhere behind the bluff body that generates the wake. Although RFG provides a robust and promising way of doing LES of complex geometry flow, it is still desirable to include the body or, at least, part of the body in the simulations, to study flow dynamics immediately after the body. There are many advantages of this approach among which are:

1. The wake flow is more realistic. At least a portion of the influences of the body 
are calculated explicitly. Thus the reverse flow behind the body, if it exists, or the nonstationary periodic flow can be taken care of directly.

2. It is more realistic for embedding a bubble dynamic simulation because most of bubbles are generated near the wall.

This will provide a state of the art study for LES of ship wake flows.

The ship model surface as well as the wavy free surface were obtained by interpolation the computational domain data from a RANS calculations (Stern and Wilson, 2000). The surface of the resulting domain for LES is presented in Fig. 7.5.

The ship model was called DTMB model 5512, a 3.048m length model scaled from DTMB model 5514. The Reynolds number $(R e)$ is $4.65 \times 10^{6}$, and the Froude number $(F r)$ based on the hull length was 0.28 .

The simulation domain started from $x / L=0.7$, the cross-section at $70 \%$ length of the ship hull. Again $x / L=0$ was the ship bow, and $x / L=1.0$ was the ship stern. The inflow boundary was specified in the same way as in the previous cases, i.e. using RFG together with RANS simulation.

The computational domain size was $1 \times 0.3 \times 0.6$ (given in non-dimensional units in ship length) in $\mathrm{x}, \mathrm{y}$ and $\mathrm{z}$-directions, respectively. The grid size was $194 \times 66 \times 98$. Non-uniform grid was used in both $\mathrm{x}$ and $\mathrm{y}$ directions.

The boundary conditions were exactly the same as the previous cases except that the no-slip wall boundary condition was applied on the ship hull surface.

The length scale and the time scale used in RFG were calculated locally by using turbulent kinetic energy $(k)$ and specific dissipation rate $(\omega)$

$$
l=k^{1 / 2} / \omega
$$




$$
\tau=1 /\left(C_{\mu} \omega\right)
$$

where $C_{\mu}=0.09$ is a empirical constant.

The Smagorinsky SGS model and second order central differencing scheme were used. Van Driest wall damping was applied in the Smagorinsky model to reduce the near-wall eddy viscosity in which the length scale takes the form (Piomelli et al., 1988):

$$
l=\left[1-e^{\left(-y^{+3} / A^{+3}\right)}\right]^{1 / 2}\left(\Delta x_{1} \Delta x_{2} \Delta x_{3}\right)^{1 / 3}
$$

where $A^{+}$is a constant taken to be 25 .

\subsection{Results and Discussion}

\subsubsection{Ship wake with flat surface}

The mean inflow boundary data was obtained by slicing the RANS solution at $x / L=$ 1.05 plane with visualization software, Tecplot, and then interpolating those data to the inlet plane of the computational domain. The mean velocities after the interpolation are presented in Fig. 7.6 to Fig. 7.9. In Fig. 7.10 the interpolated turbulent kinetic energy is shown. The two separated identical circle-like flow profiles are because of the special body structure at the bottom of the ship bow (see Fig. 7.2). By using RFG and the mean flow on this plane, the turbulent inflow boundary were re-constructed. Fig. 7.11 to Fig. 7.14 present the instantaneous velocities on this plane. Clearly, many small scale turbulence eddies can be seen. Due to the use of a constant length scale, the superimposed eddies are more or less homogeneous.

The streamwise vorticity contours at different planes are shown in Fig.7.15 and the contours of the vertical component of vorticity are shown in Fig.7.16. Fig. 7.15 shows that concentrated vorticity decreases with axial distance, and in the far wake vorticity is 
only concentrated near the free surface. Some of this rapid decay may be partially due to the grid expanding towards the outlet plane, while the size of the wake increases in axial direction of the flow. As a result of this, many turbulence structures may have died out prematurely in the simulations. It is interesting to note the two distinctly concentrated vorticity streaks away from the center line of the wake (Fig.7.16). The streamwise velocity contours at a plane near the free surface are shown in Fig.7.17. Due to the lack of experimental data for this wake, a quantitative judgment of the predictions is difficult. However, based on the other simulations (Hyman, 1995; Paterson et al., 1998), these results seem to be reasonable. As the width of the wake increases, turbulence decays. In the far wake only larger turbulence structures can been seen. This is due to the combination of two reasons: (a) coarser grids applied in the far wake; (b) the small turbulence structures contain significantly less energy so that they can only last for shorter time compared to the larger turbulence structures.

To test the effect of the grid size on the flow field resolution, the grid number was doubled in $\mathrm{x}$ (streamwise) direction and retaining the same grid in the other directions as in the previous run. In Fig. 7.18 more detailed turbulence structures can been seen clearly as compared to the coarser grid solution (Fig.7.16).

The effects of different numerical schemes and SGS models were also tested in this study. The resolved portion of the turbulent kinetic energy (TKE) for different schemes and different grid sizes is depicted in Fig. 7.19. The RANS solution is also shown in this picture for comparison. The resolved TKE from fine grid is higher than that of the coarse grid as expected. Central differencing discretization with Smagorinsky model gives better results than the other schemes. From this figure it can also be seen that there is not much difference in the results when the QUICK scheme is used with or without SGS model. Moreover, the resolved TKE is lower than that of central differencing with Smagorinsky model. It means that the QUICK scheme gives even higher numerical diffusion than the Smagorin- 
sky model. This is mostly due to grid resolution. For more detailed information about the comparisons of numerical schemes and subgrid-scale models the reader is referred to Shi et al. (2000a). One uncertainty in the computations is presented by sinusoidal-like distribution of TKE in the near wake. It may be because of the existing surface wave (not accounted directly here). When the wave descends towards the bottom of the domain, it creates a constriction with flow passing through a small area. Thus both the velocity and TKE are higher at this region. To prove this deduction, the profile was checked with the wave profile of the RANS calculation. The peak region of $k$-profile does indeed correspond to the descending wave. It is interesting to note that the present calculations also provide a similar trend although no surface wave profile was applied. This indicates that some wave information may be present implicitly in the inflow boundary.

In Figures 7.20-7.24 the velocity vectors on different vertical cross sectional planes are presented. Clearly, the large scale turbulent eddies (vortices) are captured, which can play an important role in bubble dynamics. These flow structures are not apparent in the inlet conditions (see Fig. 7.6). The prediction of outward flow near free surface, and the streamwise evolution of the vortices are very encouraging indications of the success of the present LES approach.

The vorticity contours on different vertical planes shown in Fig.7.25, Fig.7.26 and Fig.7.27 are indicative of the degree of resolution of the calculations. These structures are hard to see in Figures 7.20-7.24 due to relatively small magnitude of the velocity vectors. However, to demonstrate the small weak turbulence structures, like those in Fig.7.23(a), the areas containing turbulence structures corresponding to those in the vorticity contour plot have been enlarged and depicted them in Fig.7.23(b). A corresponding area in the vorticity contour plot is also included in Fig.7.27 for reference with the cross section taken at $\mathrm{x} / \mathrm{L}=$ 1.2. In the velocity vector plot the weak vortices can not be seen due to scale difference, whereas the contour plots do show small structures. Figures 7.26 and 7.27 show again that 
much of the vorticity is concentrated near the free surface, and there the two large counter rotating vortices originally from the ship hull on two sides of the wake have been retained in LES.

\subsubsection{Ship wake with sinusoidal free surface}

This case is designed to test the influence of the wave surface on the distribution of the turbulence kinetic energy. The flow field at the inflow boundary is the same as the previous case.

The free surface is represented by a free-slip sinusoidal wall. As shown in Fig. 7.28, the surface has a significant influence on the turbulent kinetic energy distribution, although the amplitudes of the wave here are much larger than those in RANS calculations. This seems to be in agreement with the experiments of Rashidi et al. (1992) as well as with the DNS of Lombardi and Angelis (1996). This increase may have originated from convective transport of kinetic energy towards the surface under the influence of the imposed wave profile. Of course, further studies of the turbulence/wave interaction are necessary to clarify the interaction of surface waves with turbulence.

\subsubsection{Ship wake flows including part of the ship hull with free surface waves}

The inflow boundary conditions were in the same way as in the previous cases. The mean flow field is shown in Fig. 7.29 to Fig. 7.33. The turbulent kinetic energy is presented in Fig. 7.34. In this figure, the presence of the wall boundary layer is apparent. To see the details in the boundary layer, area $A$ in Fig. 7.30 and Fig. 7.34 is enlarged to show the streamwise velocity and turbulent kinetic energy in Fig. 7.30 and Fig. 7.35, respectively. The flow in the boundary layer flow is expected to be strongly anisotropic and nonhomogeneous. Thus the length scale and time scale should be calculated locally. Also, the anisotropic stresses should be taken into account. In this study, both time and length scales 
are computed from $k$ and $\omega$. However, because of the lack of information about the shear stresses, isotropic turbulence assumption was involved. The instantaneous flow field resulted from RFG is shown in Fig.7.36 to Fig. 7.39. It is seen that the turbulence generated is quite nonhomogeneous.

The measurements of the flow field for model 5512 are now underway at the University of Iowa. A preliminary results at a cross section located at $x / L=0.935$ was available and they were used to assess the simulations. The computed streamwise velocity at this plane is shown in Fig. 7.40 to be compared with that from the measurements, which is presented in Fig. 7.41. Qualitatively, both are comparable considering that the results from the simulations are instantaneous (not averaged) of an instance, and therefore some turbulence structures exist. However, the velocities at the other two directions, Fig. 7.42 to Fig .7.45, are not easy to compare with measurements because through averaging, the measurements smooth out all the turbulence information. Nevertheless, the distribution of the velocities is basically similar. For example, the peak of the vertical velocity is at the same location $(0.0,-0.25)$ and overall vertical velocity is upwards. Note, in the experiments or RANS simulation, the vertical direction was assigned as $z$ direction while the spanwise direction was assigned as $y$ direction, different from the present study. Comparisons of the velocity vectors at this plane between measurements and simulations are presented in Fig. 7.46 and Fig. 7.47. Again, the overall quality of the simulations is satisfactory.

In Fig. 7.48 to Fig. 7.50 velocity contours very near the surface plane are shown. Correspondingly, the vertical vorticity on the plane is demonstrated in Fig. 7.51. The turbulence structures reflect the core of the ship wake.

Similarly, the streamwise velocity and the spanwise vorticity at a vertical plane are shown in Fig. 7.52 and Fig. 7.53. Most of the turbulence structures are sustained near the free surface. In the stern region, reverse flow is present. 


\subsection{Summary}

Most of the results in this Chapter provide qualitative studies because very limited experimental data was available to compare with. However, valuable assessments still can be made through numerical analysis.

The RFG technique in conjunction with LES has been applied to simulate the turbulent wake flows. Ship model DTMB 5512 was used as the subject of the wake flows.

Three cases have been studied: (i) ship wake with flat free surface; (ii) ship wake with sinusoidal free surface; (iii) ship wake including part of the ship hull with free surface waves. All these cases can be considered stake-of-the-art studying of ship wakes.

The simulation resolves most of the turbulence, especially the large eddies. A distinct pair of counter-rotating longitudinal vortices exist in the wake. These type of vortices have been referred to as the "bilge vortices" (Hoekstra, 1991) which are generated by the shrinking of the ship body at the stern range.

As the turbulence intensity decays in the wake, most of the turbulence structures concentrate near the free surface.

The presence of the surface waves has significant effects on the distribution of the turbulent kinetic energy.

Including part of the ship hull in LES and using these results for far wake simulation can improve the predictions. This is more desirable for future studies because the ship hull itself is the source of the turbulence, which is always better to be solved explicitly. In addition to this the inclusion of a part of the ship hull provides benefits to the bubble dynamic research, since bubbles are influenced primarily by the ship-surface boundary layer.

One-equation SGS model has not been used in the simulation of the ship wake flows. But, based on its success in the channel and flat plate wake flows, it is believed that with one-equation SGS model even better results will be achieved. 


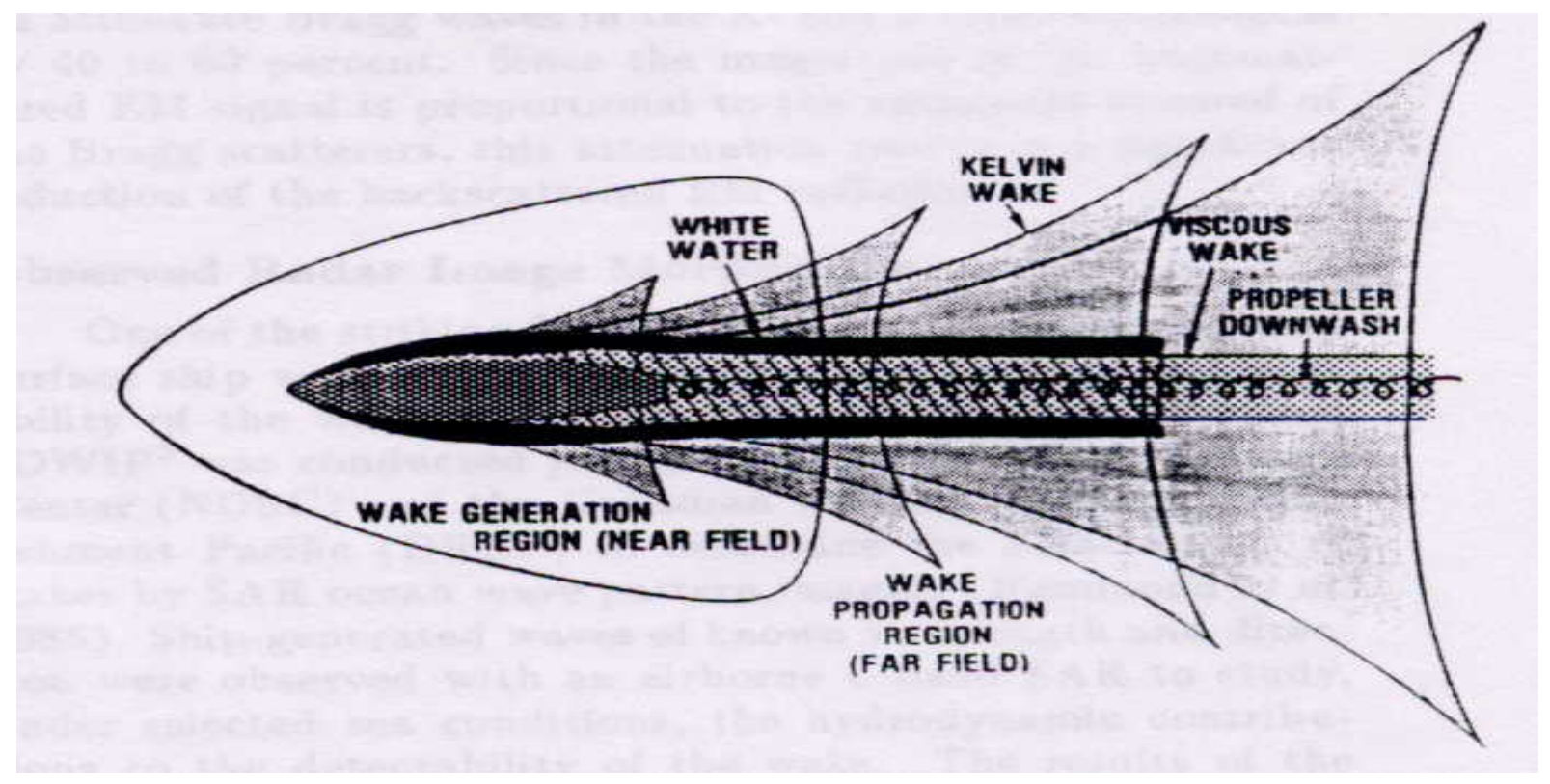

Figure 7.1: Hydrodynamic wake schematic showing major large-scale contributors to the wake (Reed et al. 1990)

Figure 7.2: Geometry of DTMD 5415 


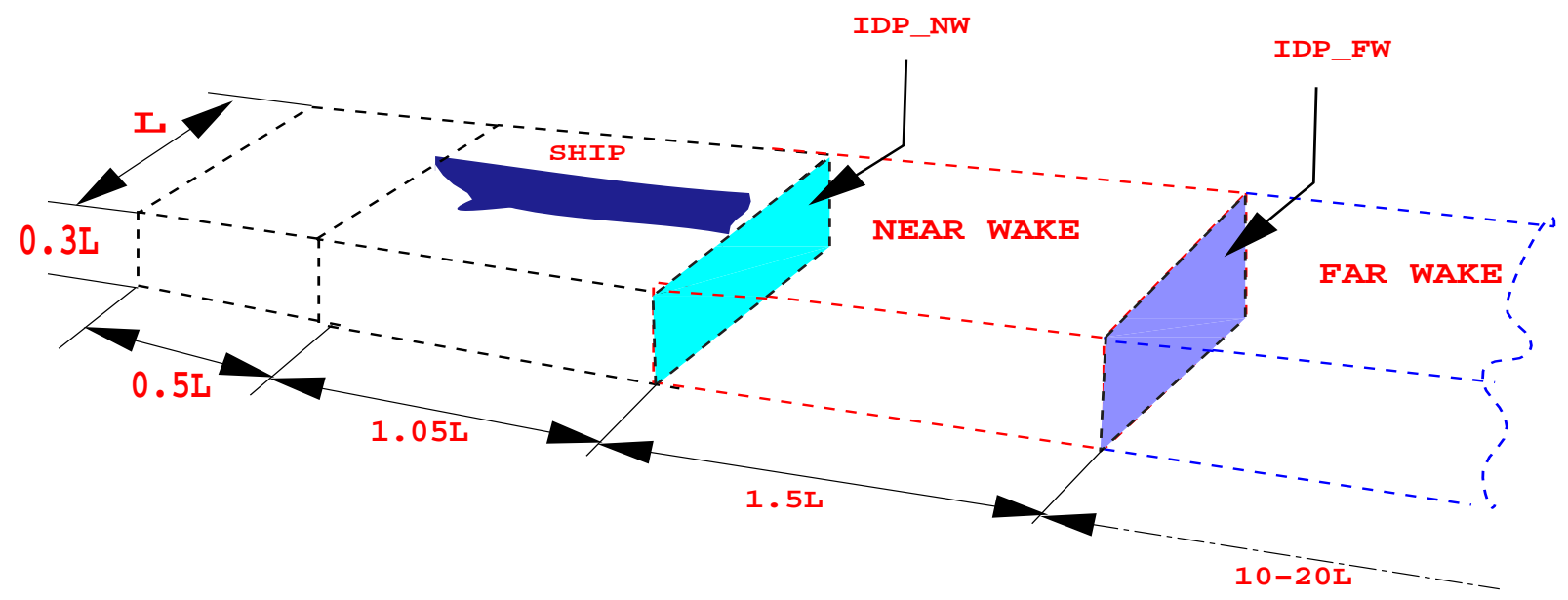

THE SKETCH OF FLOW PAST SHIP HULL

(I is the ship length)

Figure 7.3: Ship wake simulations setup 


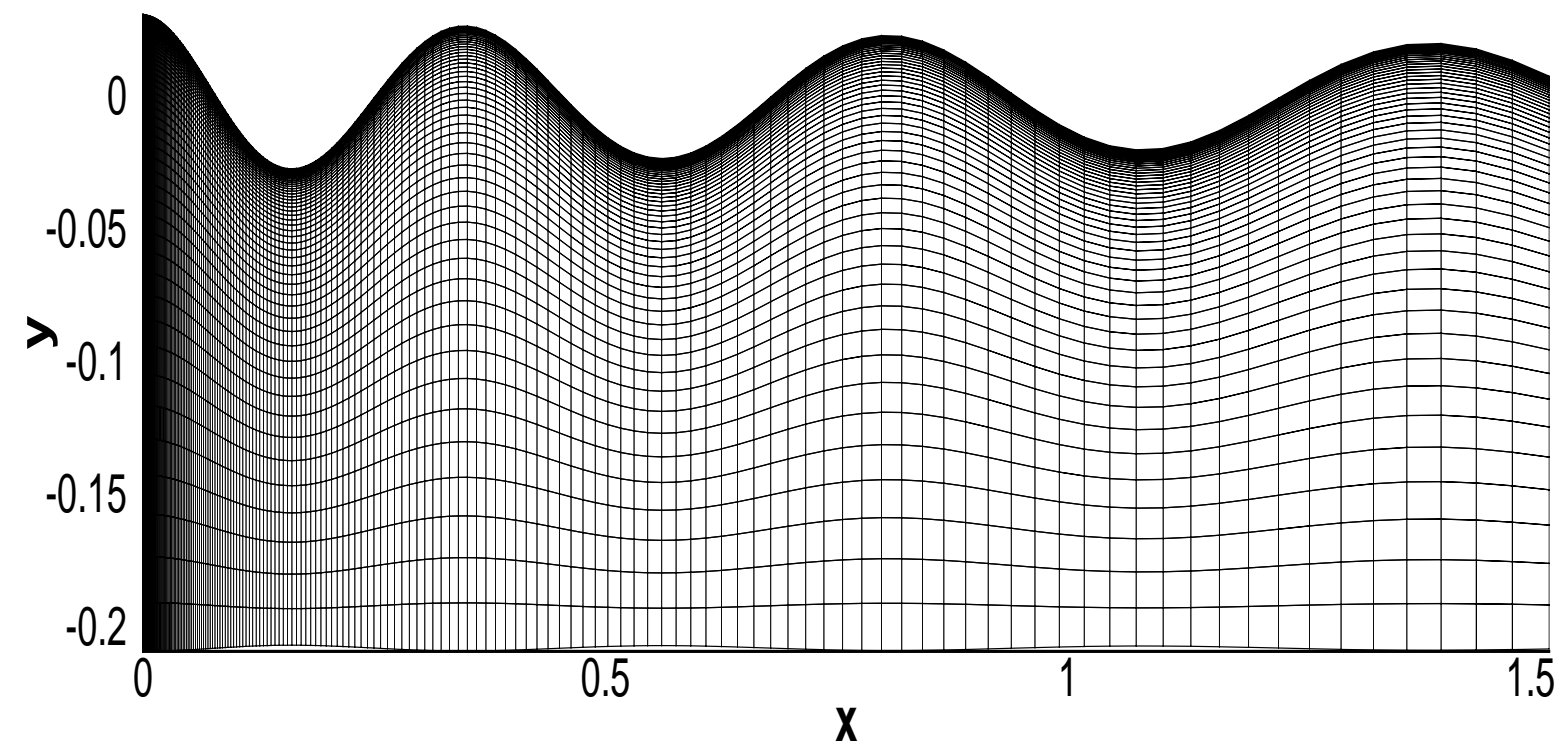

(a) $x-y$ plane

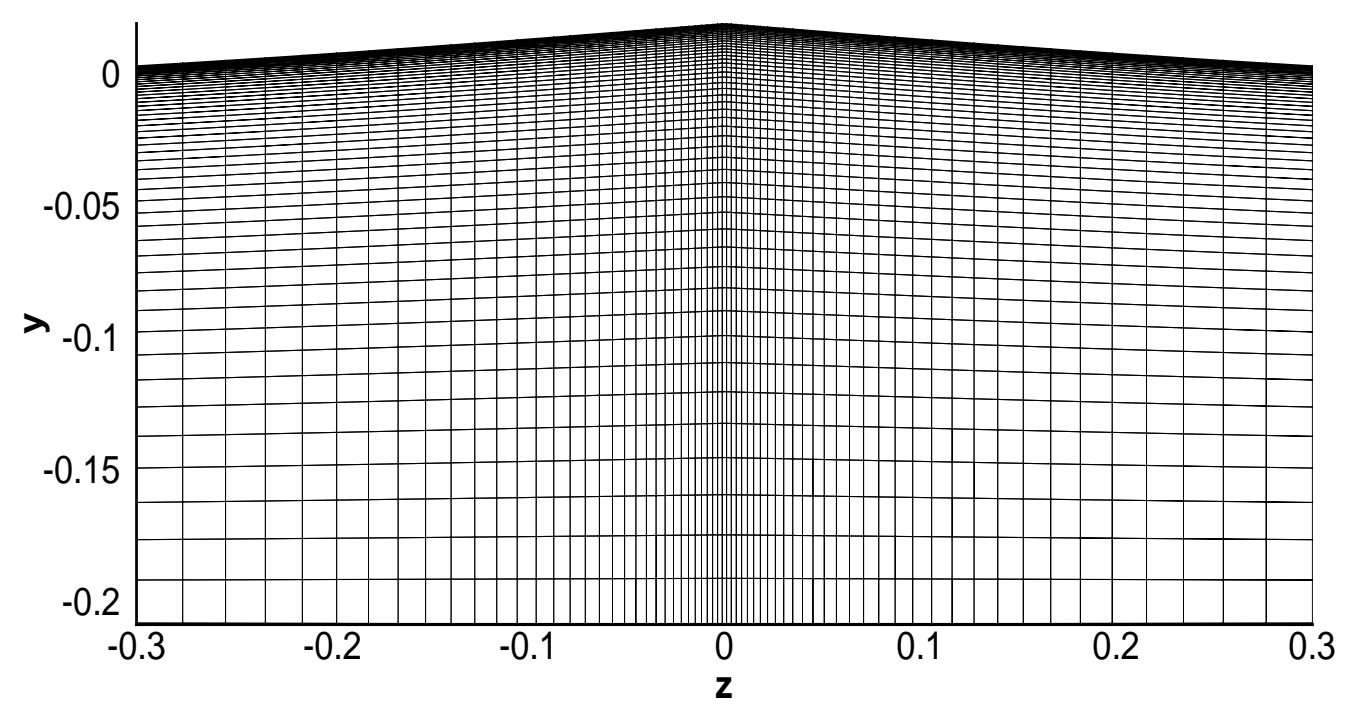

(b) $y-z$ plane

Figure 7.4: Ship wake simulations setup with surface waves 


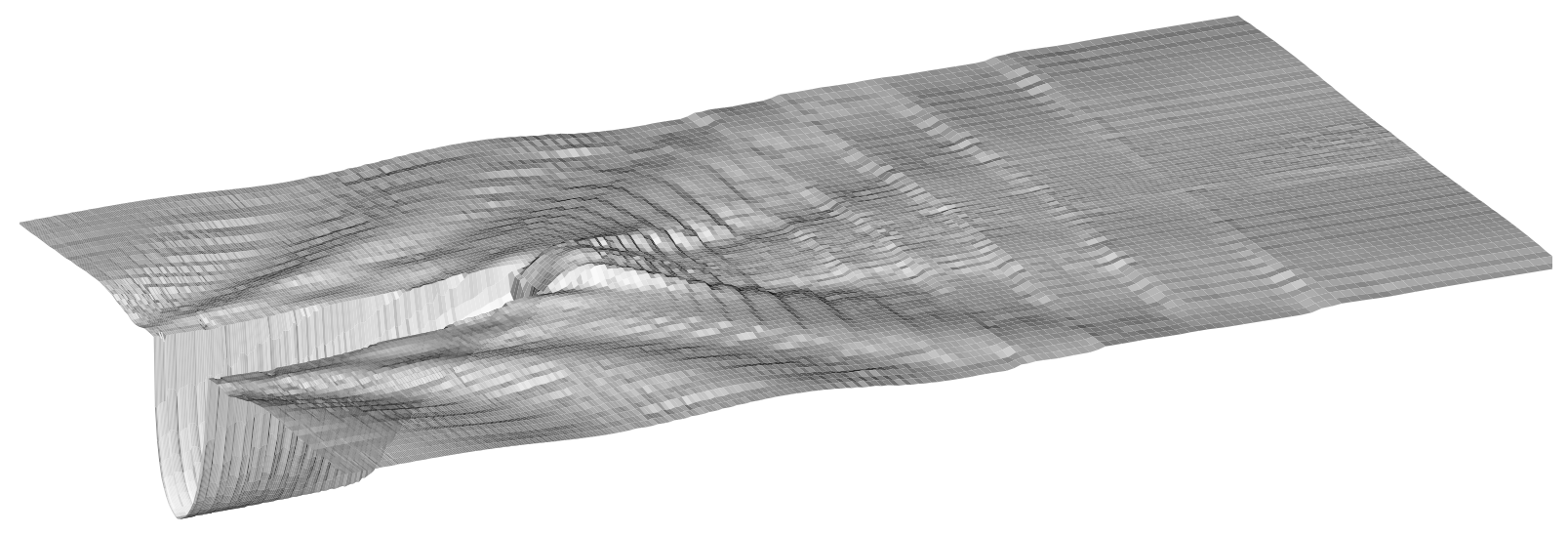

Figure 7.5: The sketch of ship wake including part of the ship hull and wave surface

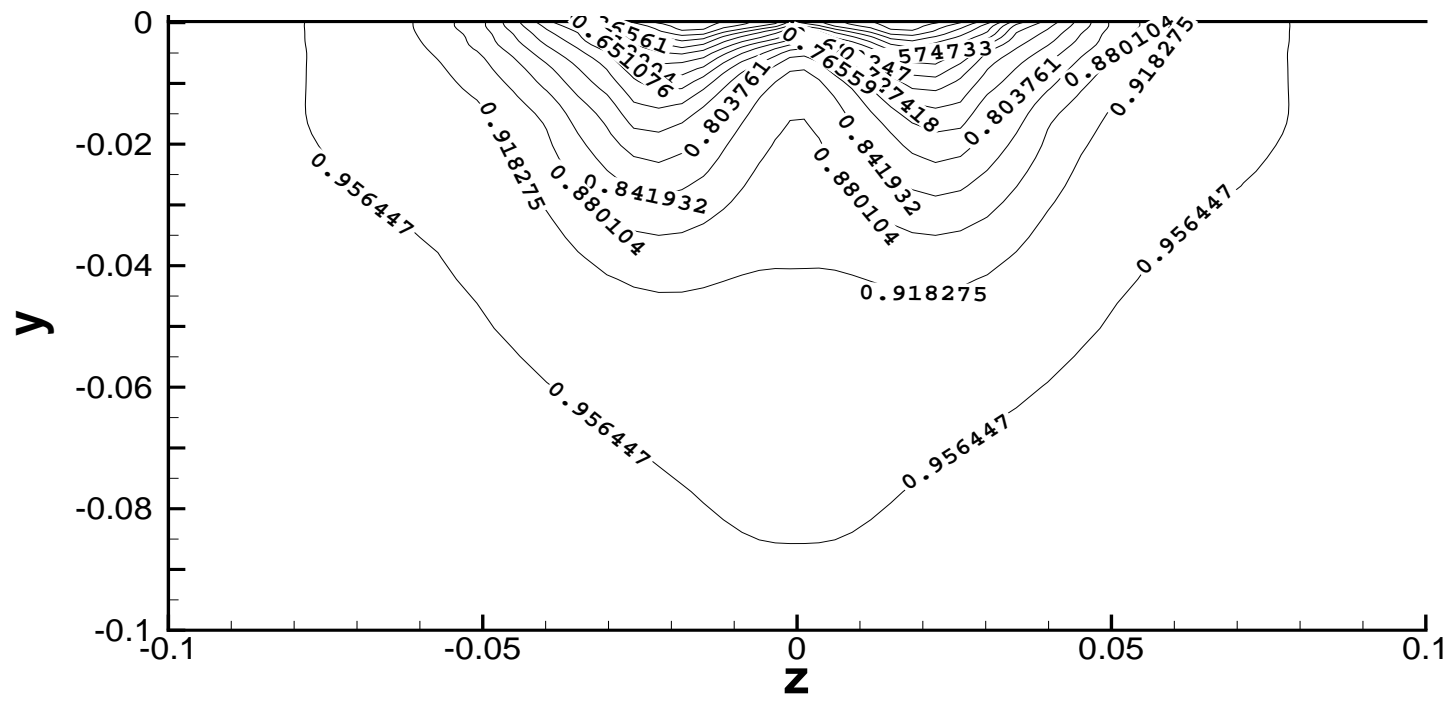

Figure 7.6: The mean streamwise velocity at the inlet plane for case 1 


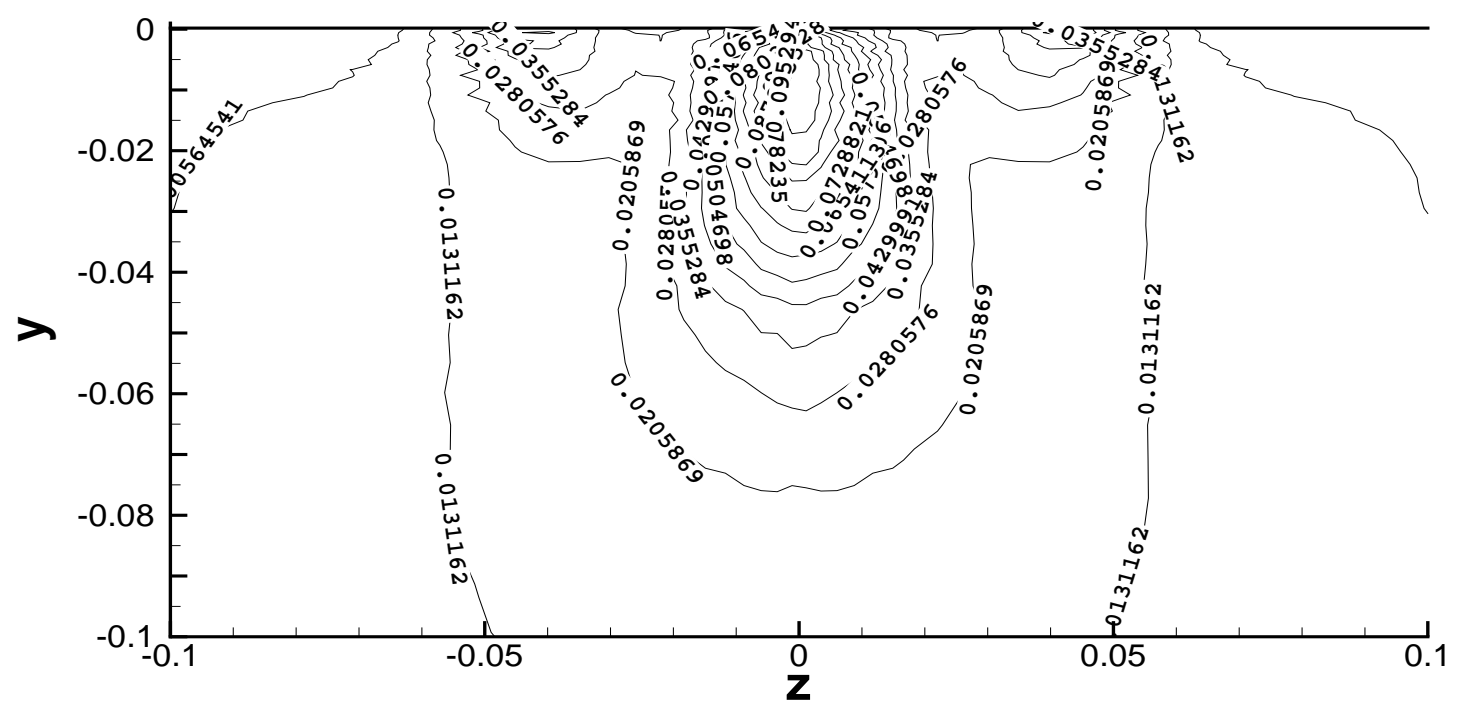

Figure 7.7: The mean vertical velocity at the inlet plane for case 1

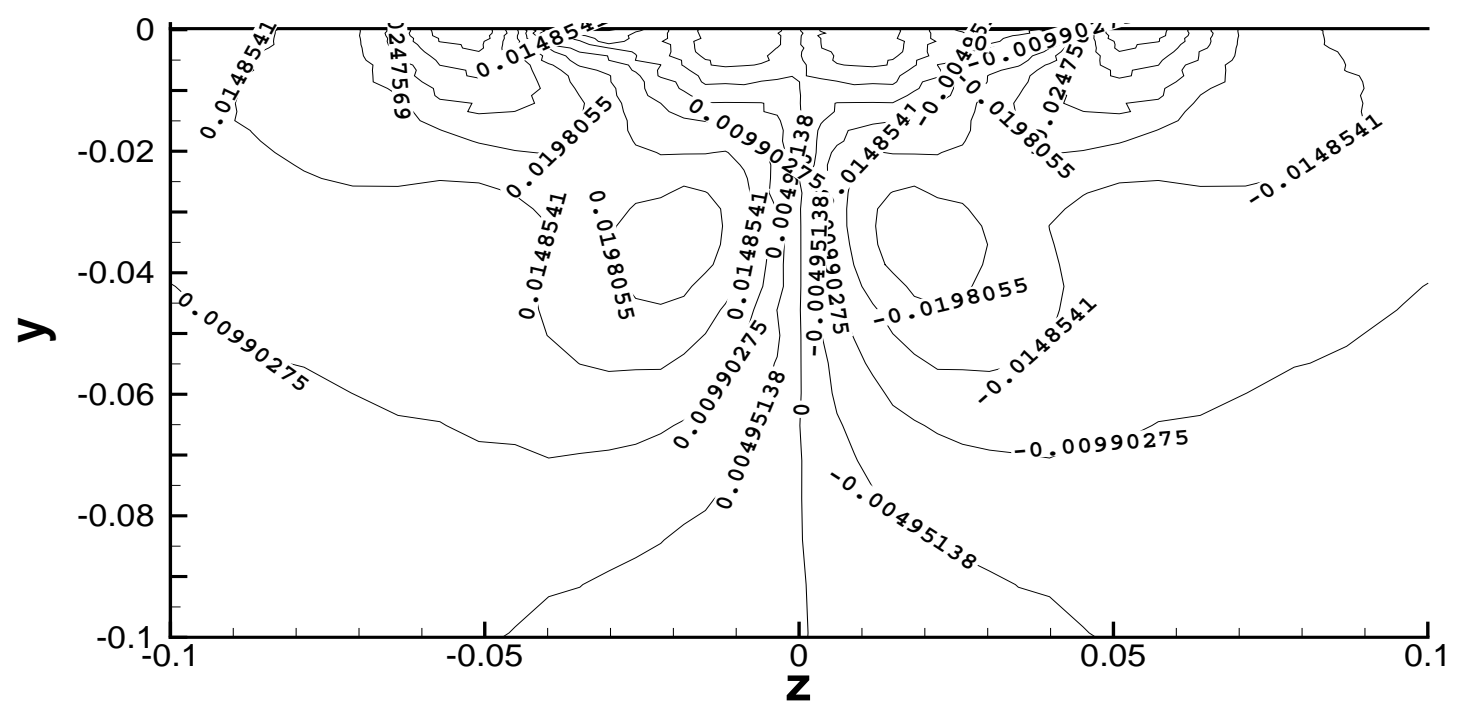

Figure 7.8: The mean spanwise velocity at the inlet plane for case 1 


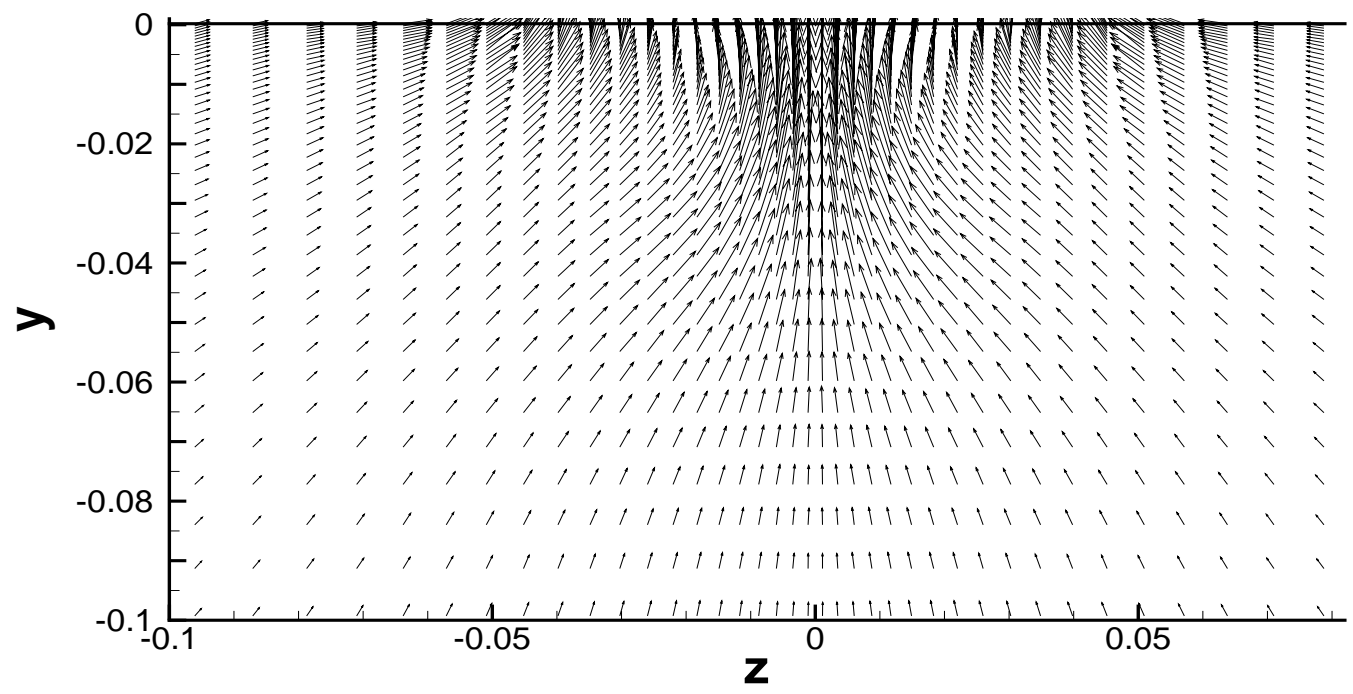

0.1

Figure 7.9: The mean velocity vectors at the inlet plane for case 1

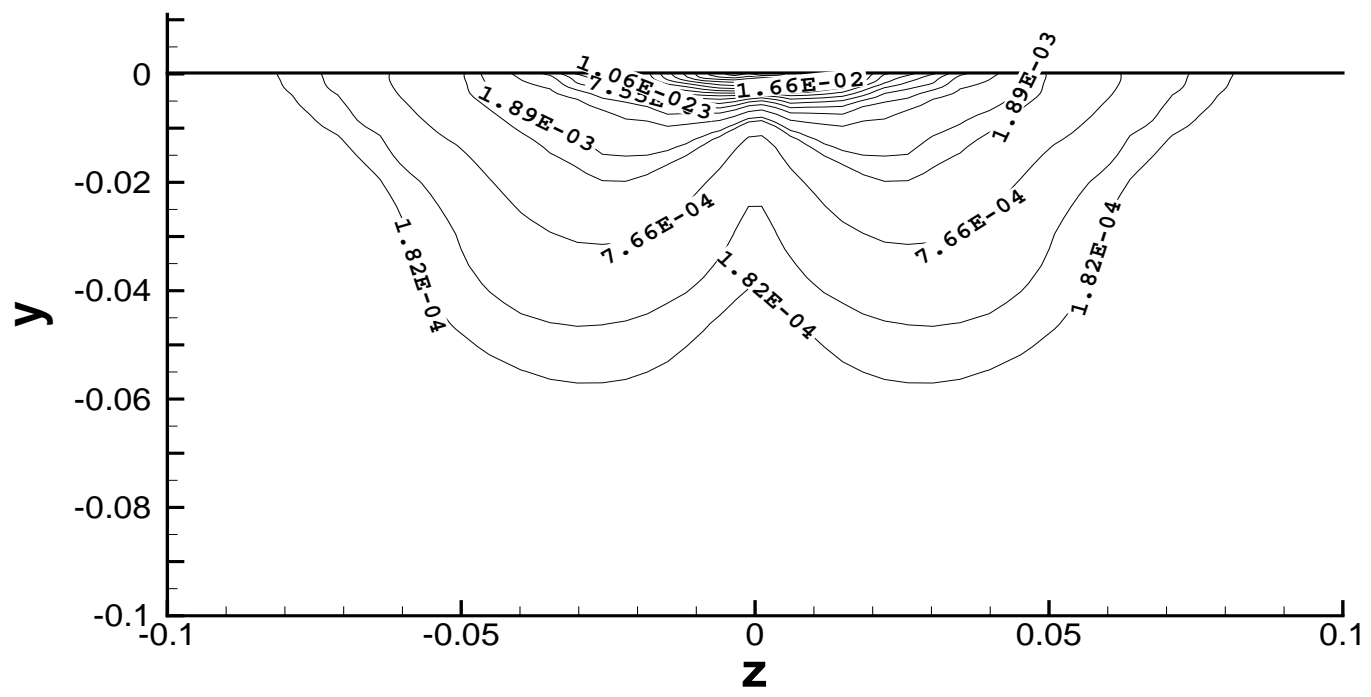

Figure 7.10: The mean turbulent kinetic energy at the inlet plane for case 1 


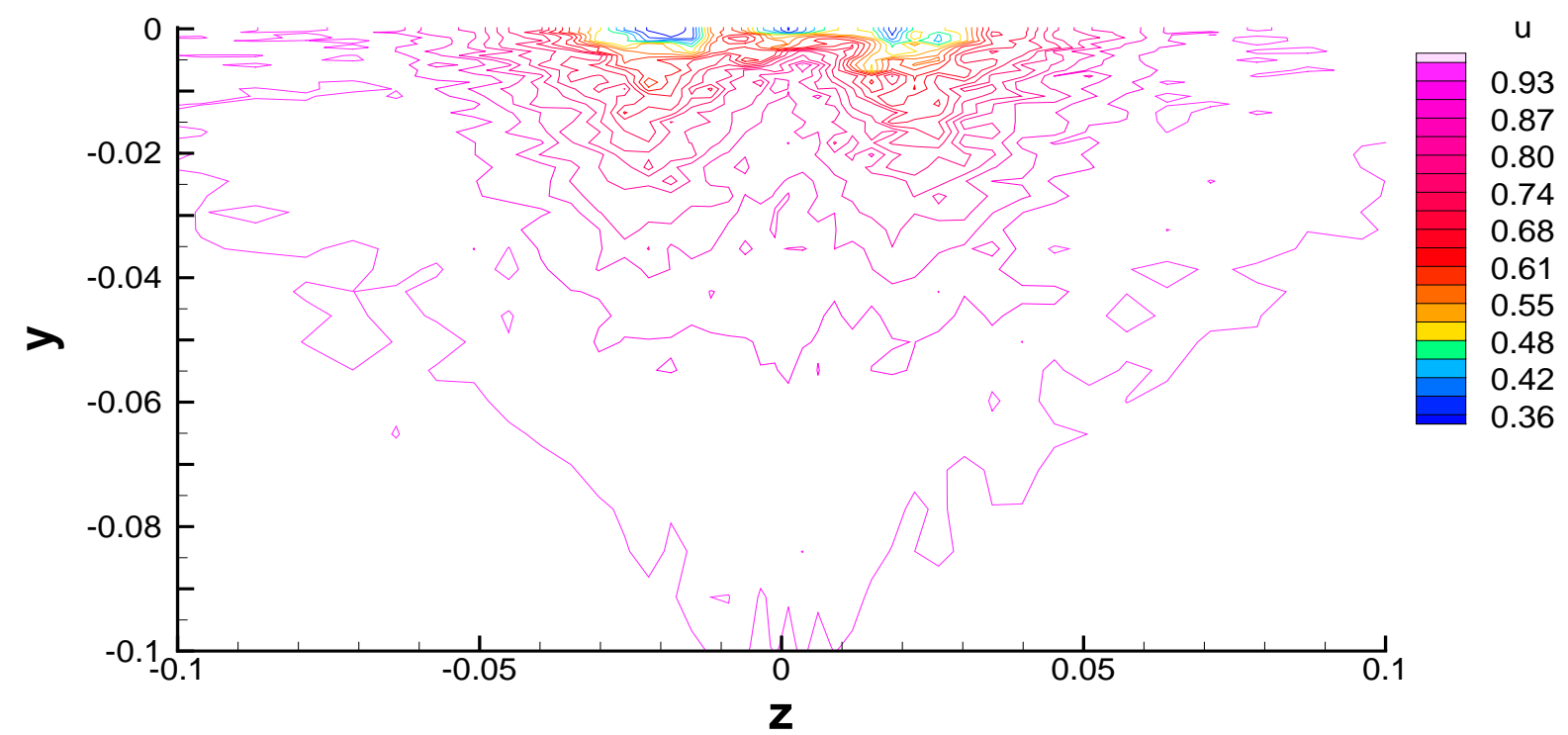

Figure 7.11: Typical instantaneous streamwise velocity at the inlet plane for case 1

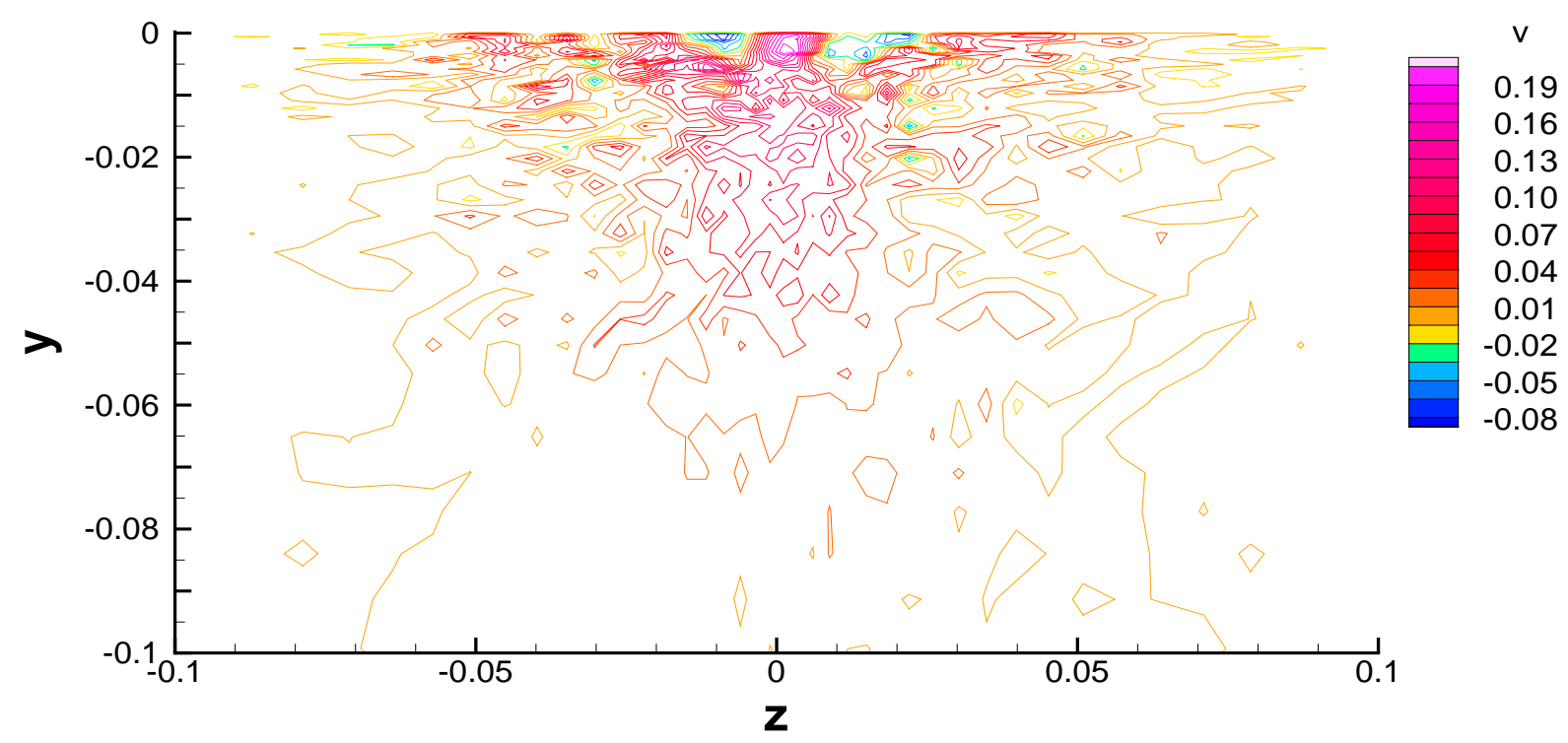

Figure 7.12: Typical instantaneous vertical velocity at the inlet plane for case 1 


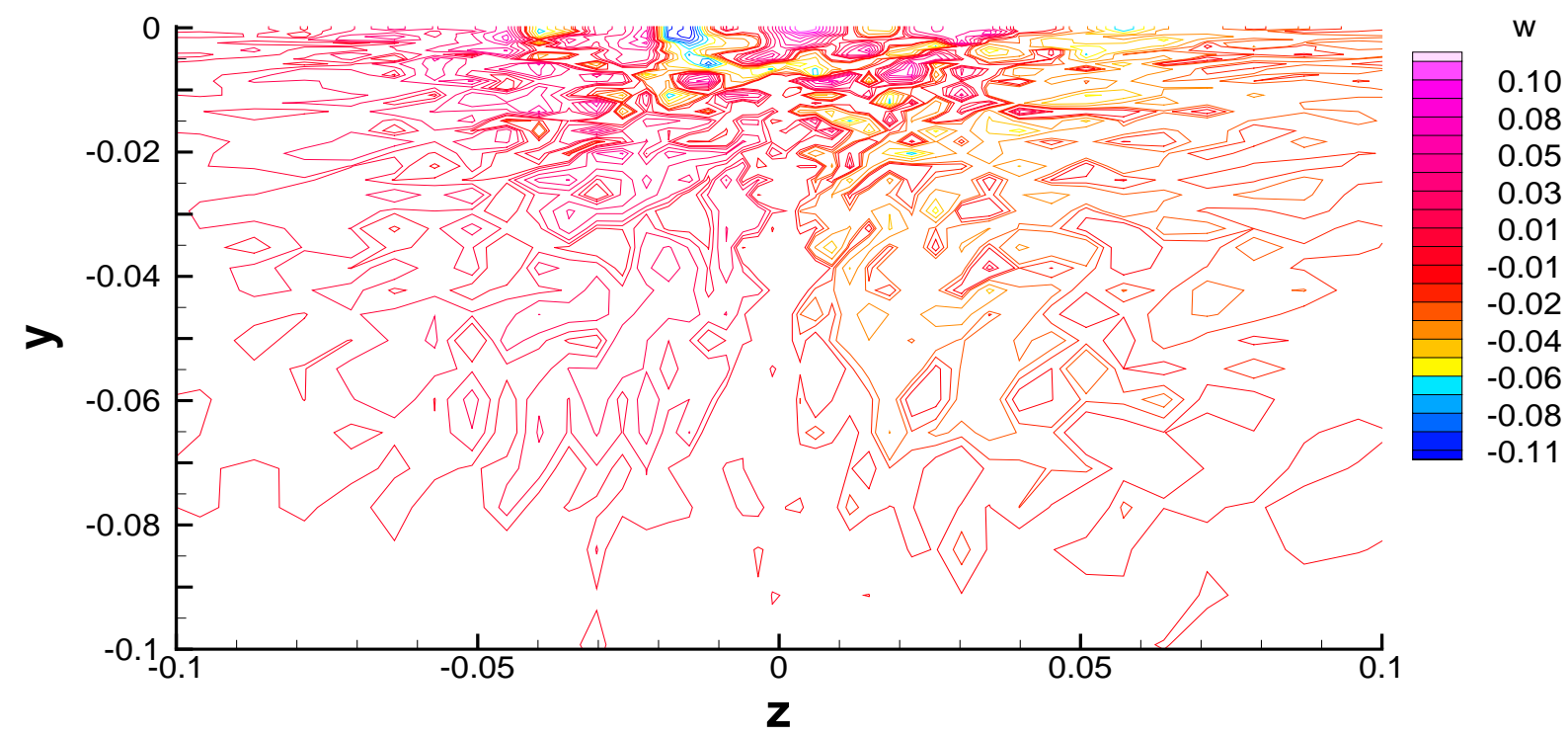

Figure 7.13: Typical instantaneous spanwise velocity at the inlet plane for case 1

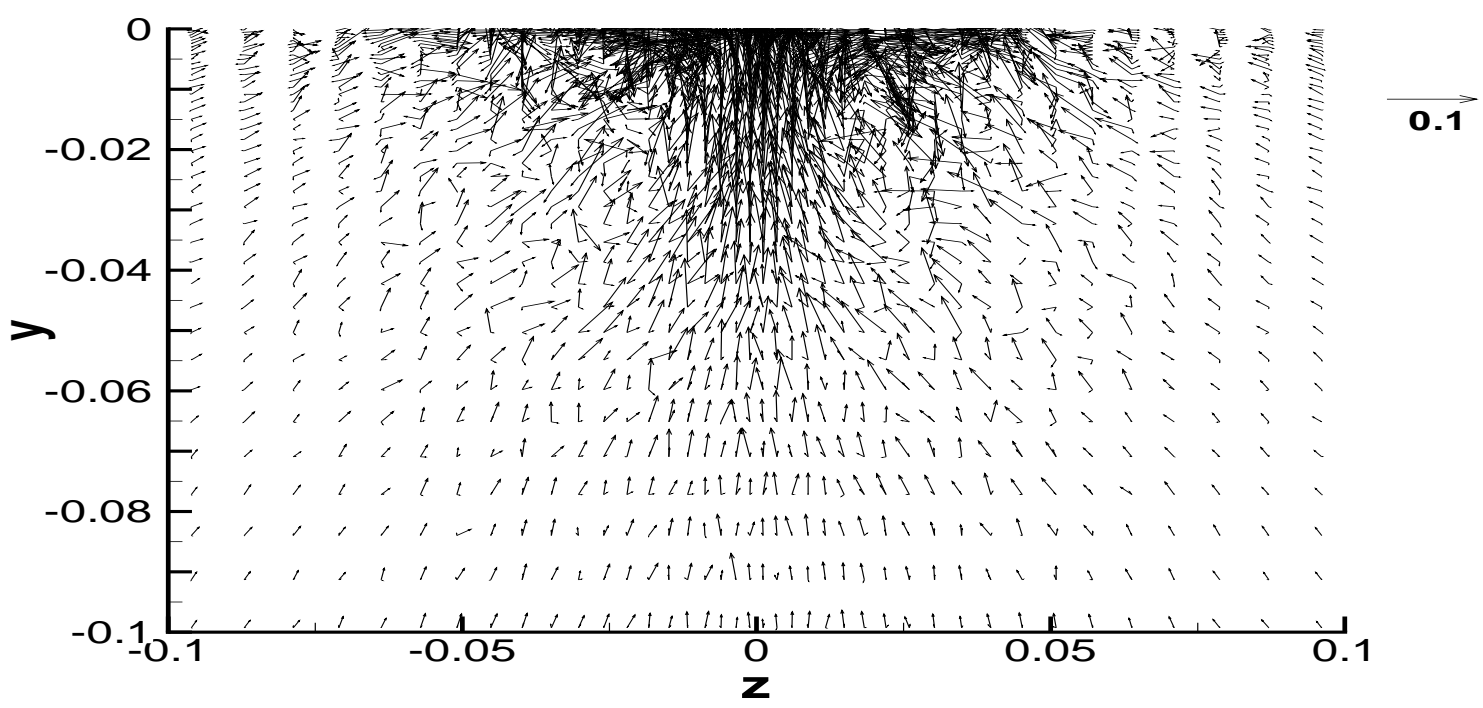

Figure 7.14: Typical instantaneous velocity vectors at the inlet plane for case 1 


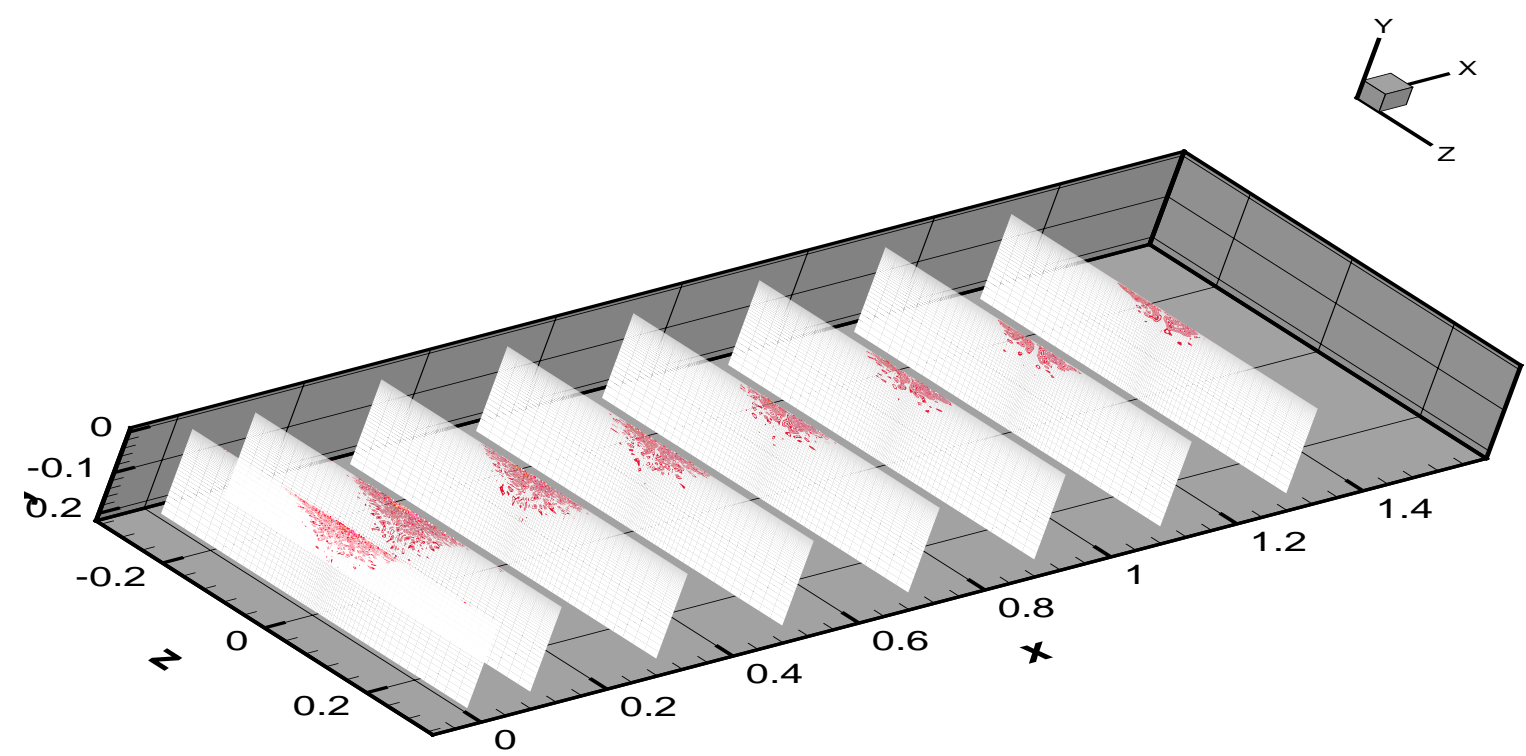

Figure 7.15: Typical instantaneous streamwise vorticity $\left(\omega_{x}\right)$ contours on different y-z plane in the ship wake

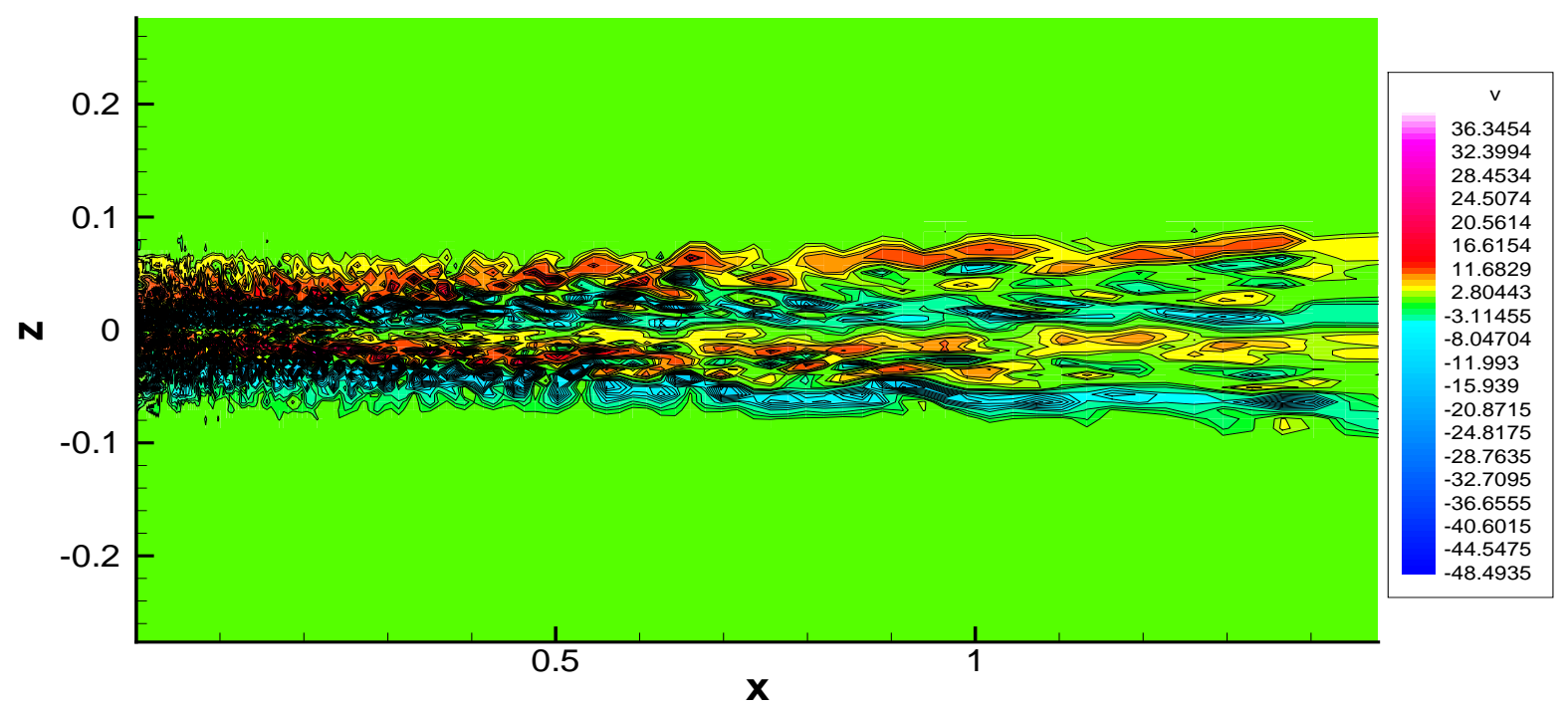

Figure 7.16: Typical instantaneous vorticity contours $\left(\omega_{y}\right)$ on a $\mathrm{X}-\mathrm{z}$ plane parallel to free surface at $y / L=-0.01$ in the ship wake 


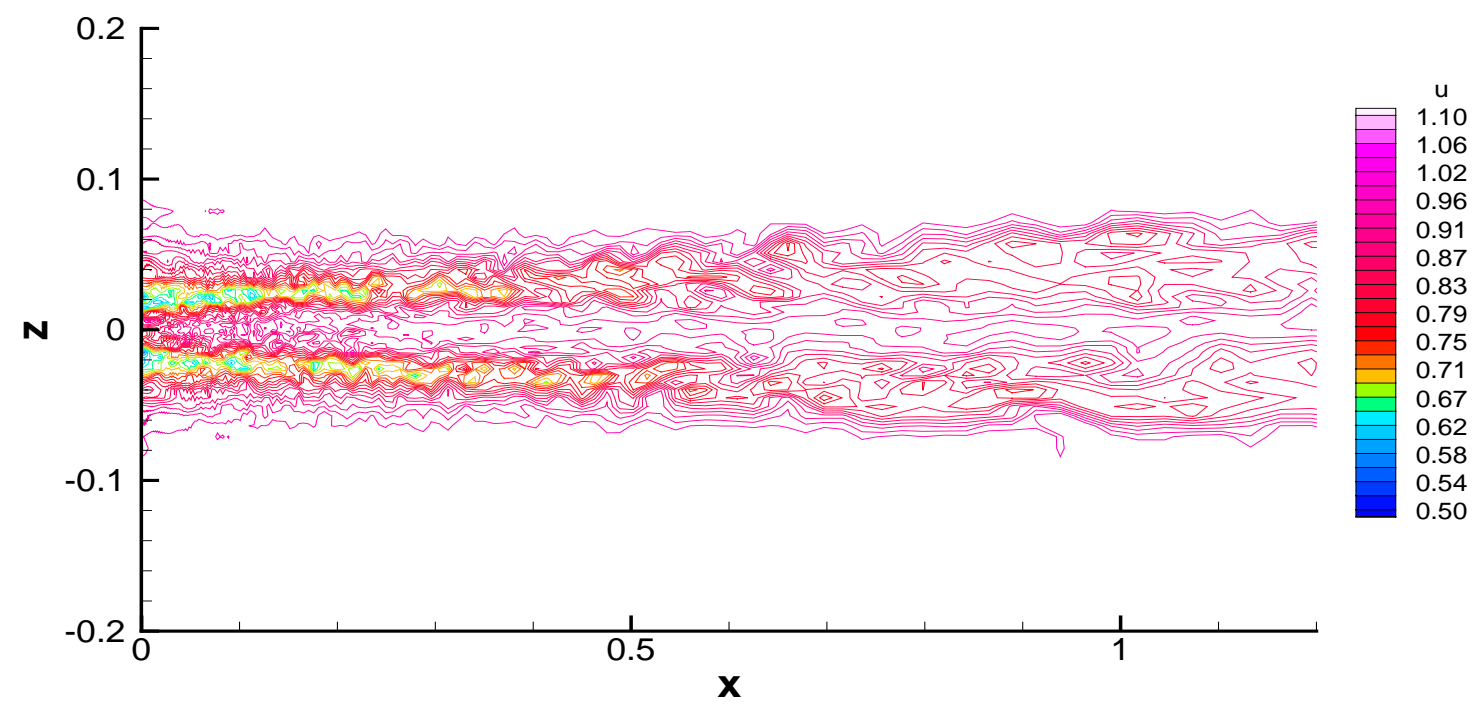

Figure 7.17: Typical instantaneous streamwise velocity contours on a $\mathrm{X}-\mathrm{z}$ plane parallel to free surface at $y / L=-0.01$ in the ship wake

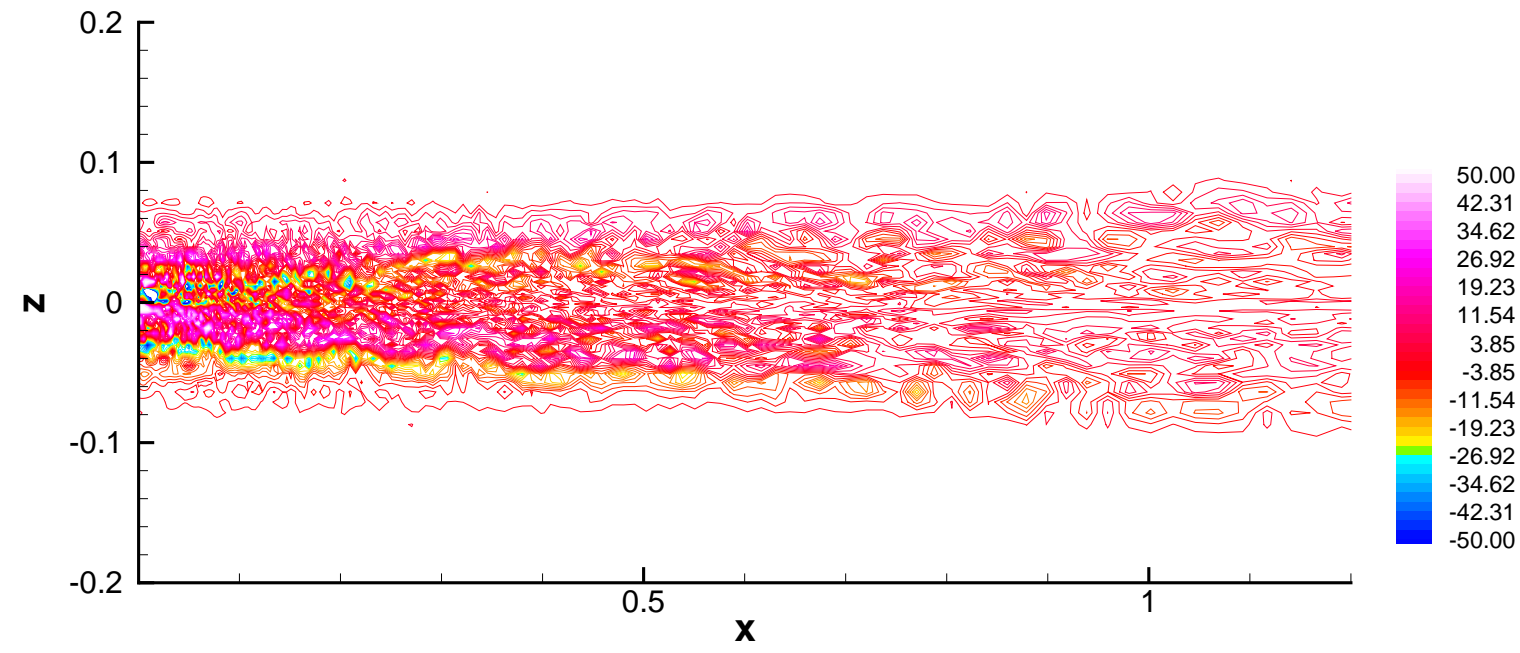

Figure 7.18: The same as Fig.7.16 but double grid number in streamwise direction 


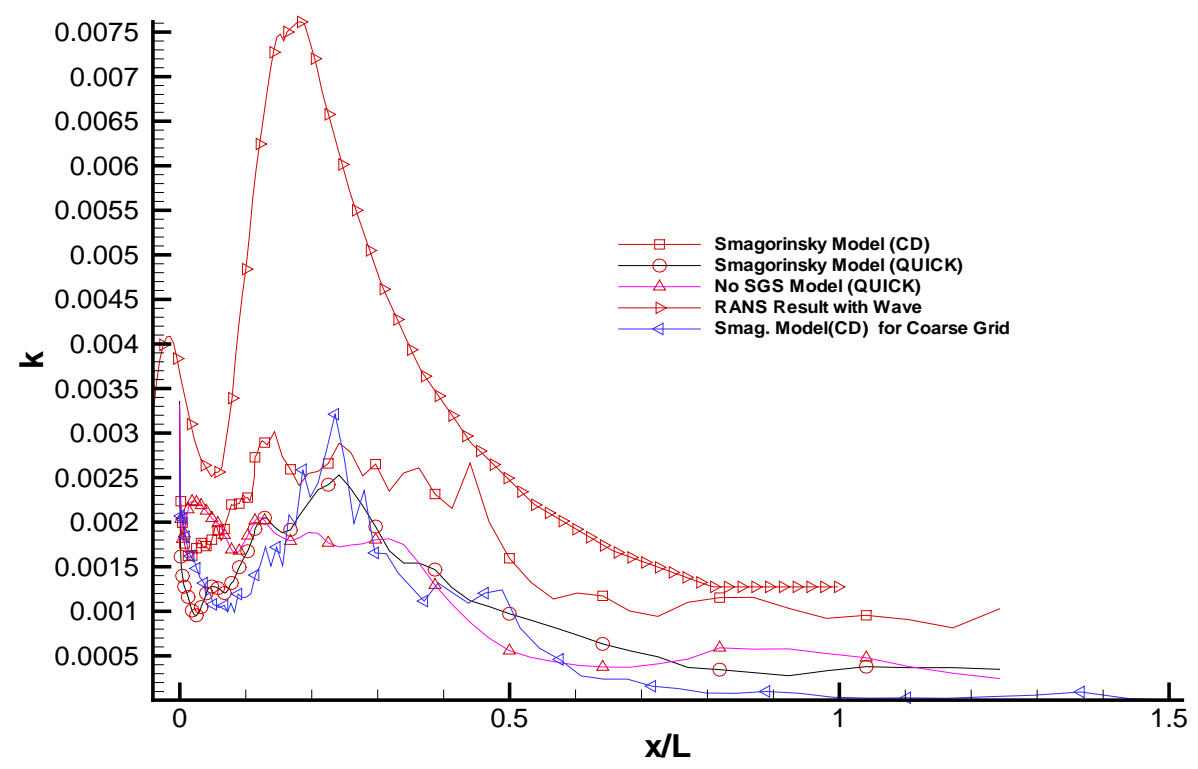

Figure 7.19: Comparison of resolved turbulence kinetic energy for different computing cases

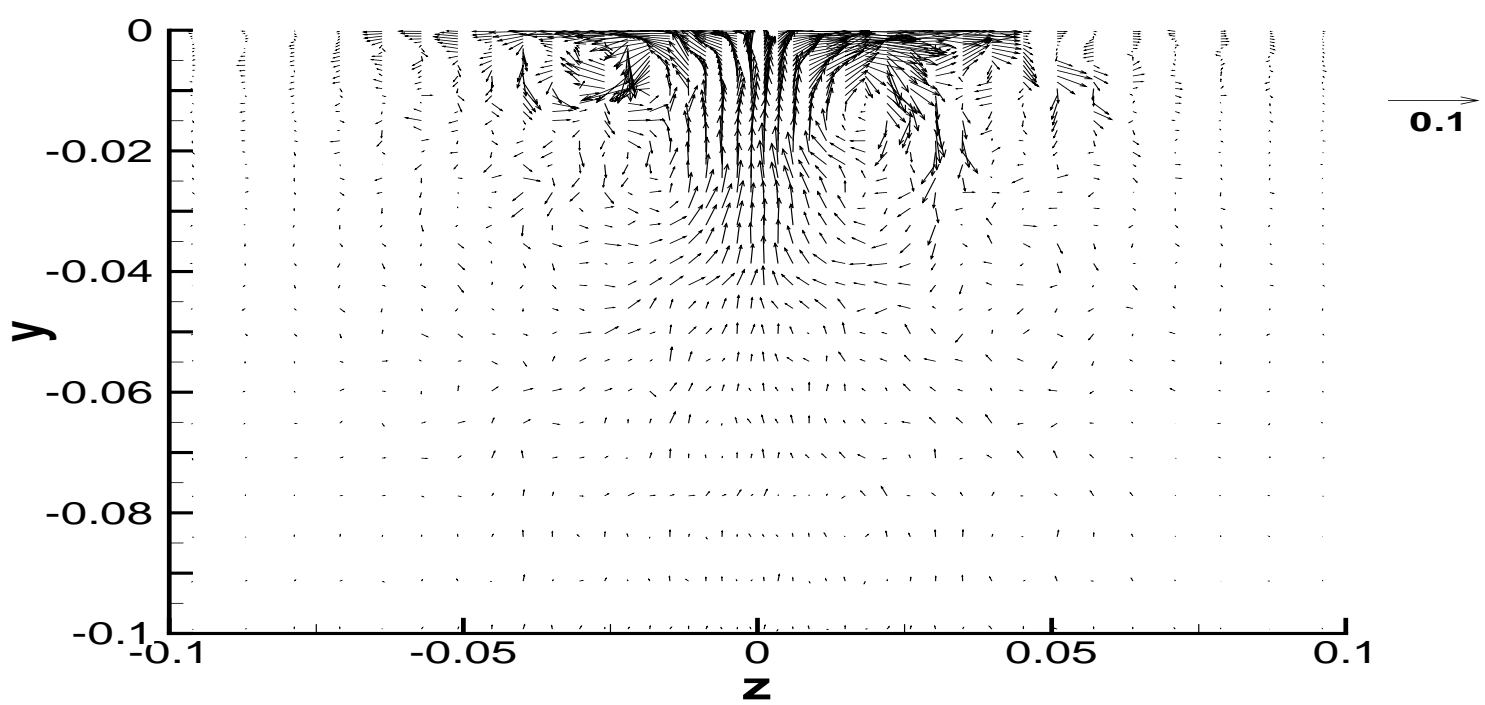

Figure 7.20: Typical instantaneous velocity vectors on $y-z$ plane at $x / L=0.2$ in the ship wake 


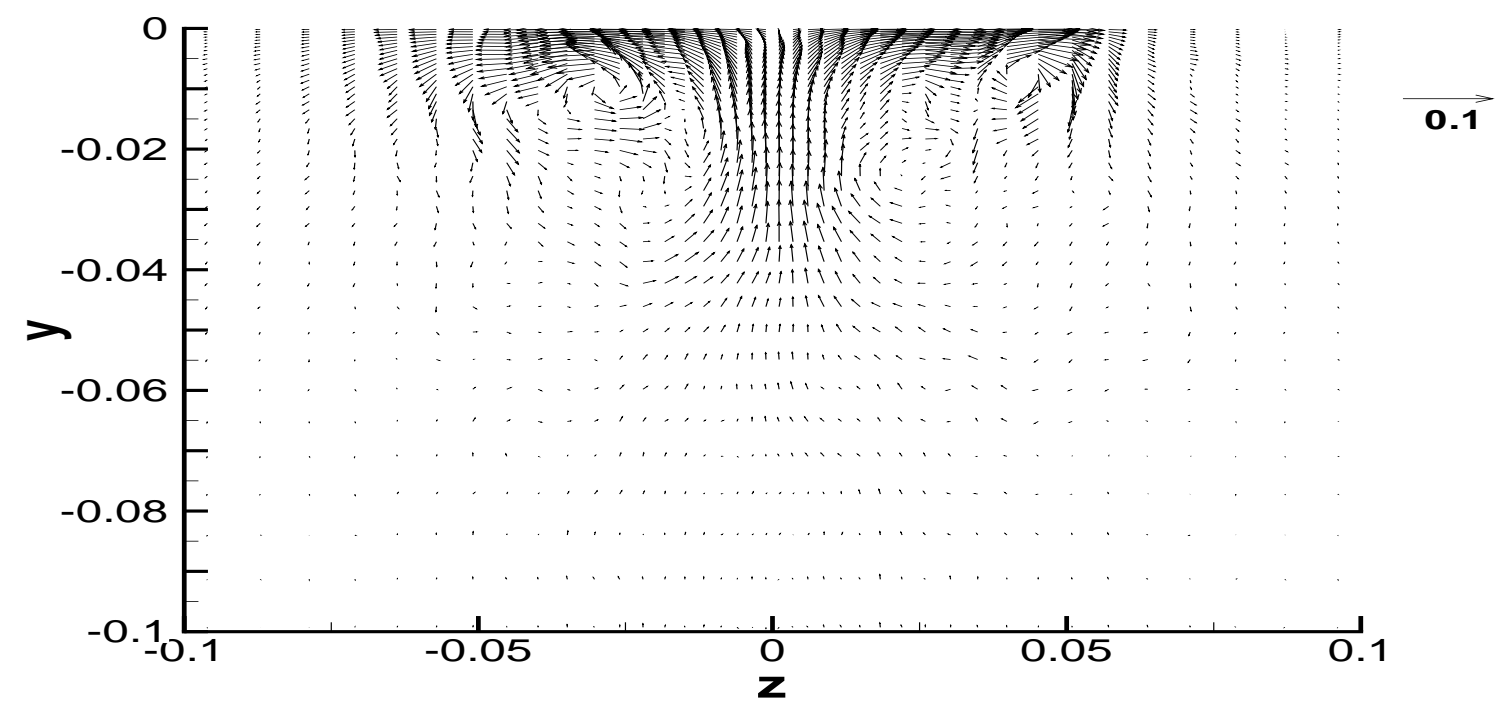

Figure 7.21: Typical instantaneous velocity vectors on $y-z$ plane at $x / L=0.6$ in the ship wake

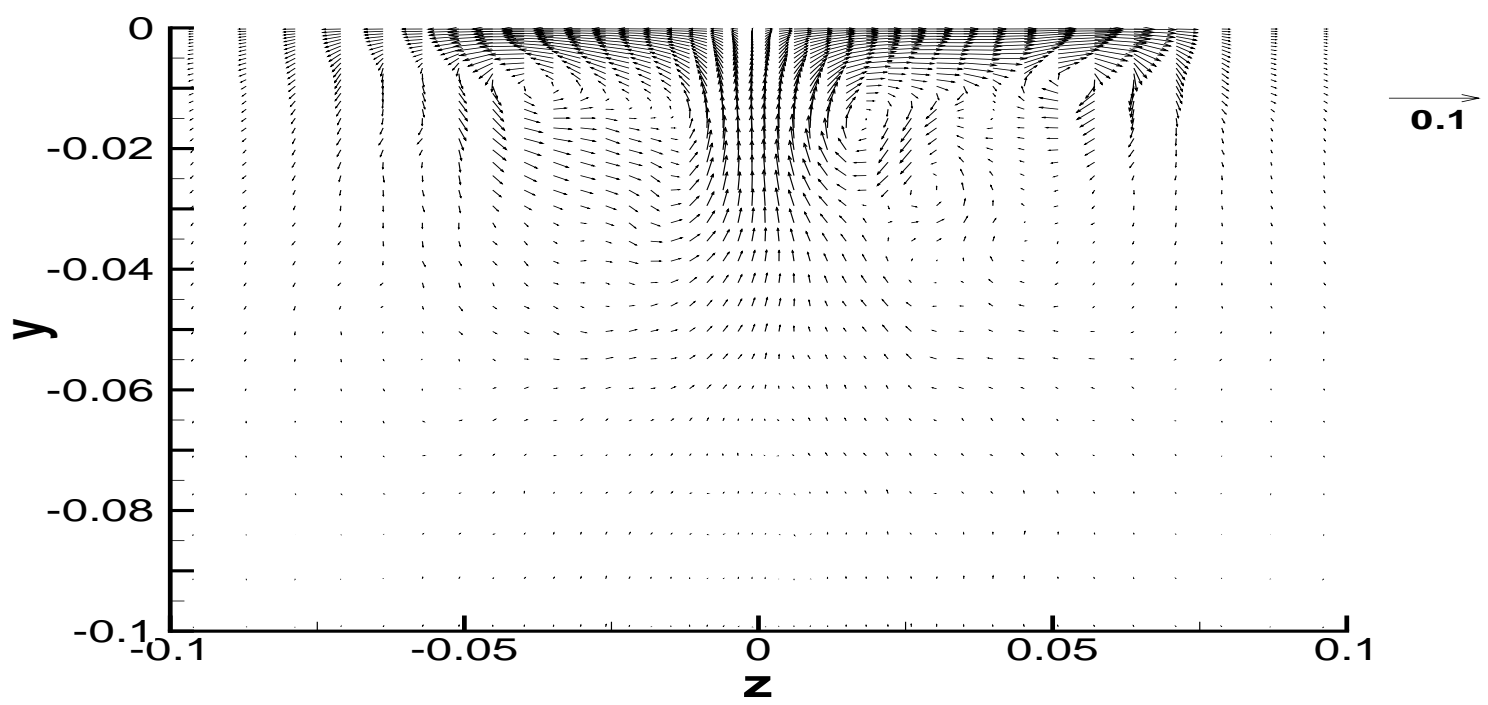

Figure 7.22: Typical instantaneous velocity vectors on $y-z$ plane at $x / L=1.0$ in the ship wake 


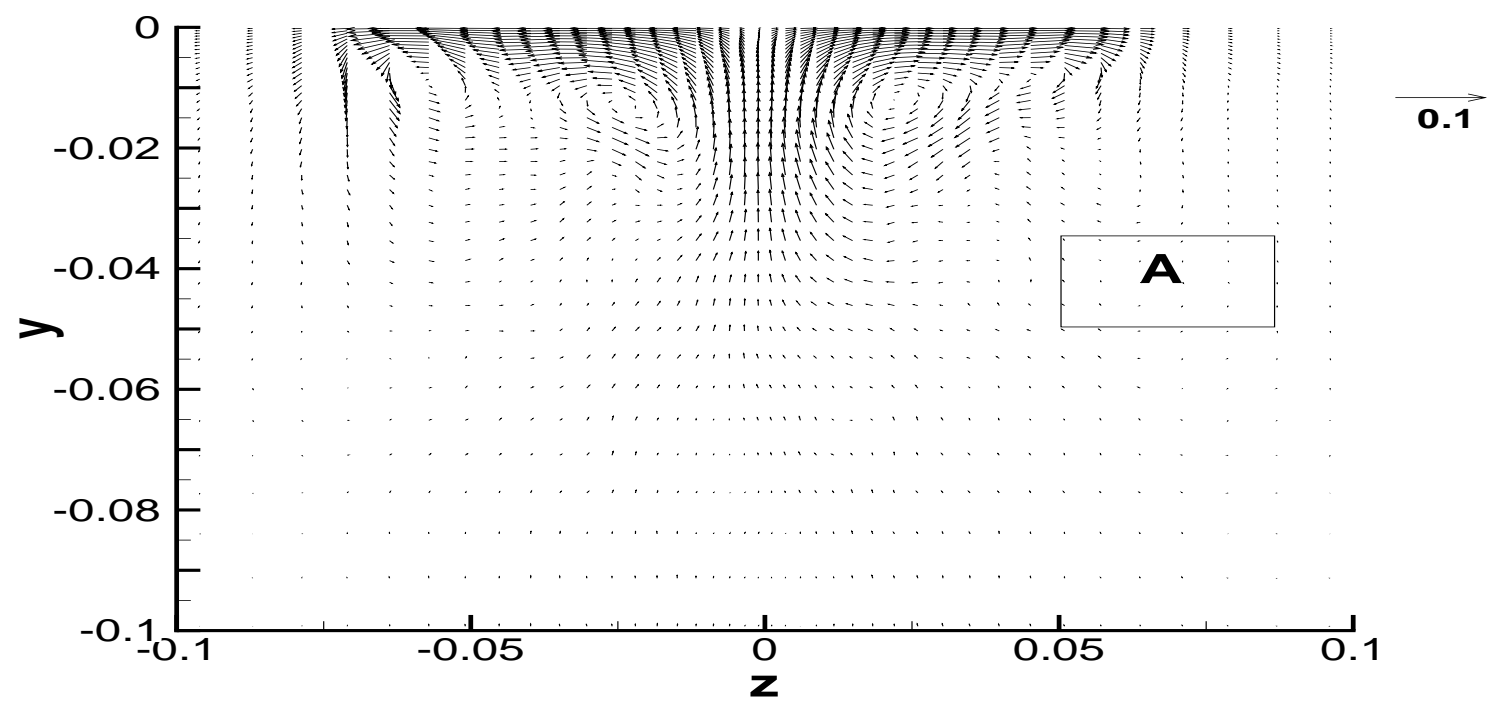

(a) The velocity vectors on $y-z$ plane at $x / L=1.2$ in the ship wake

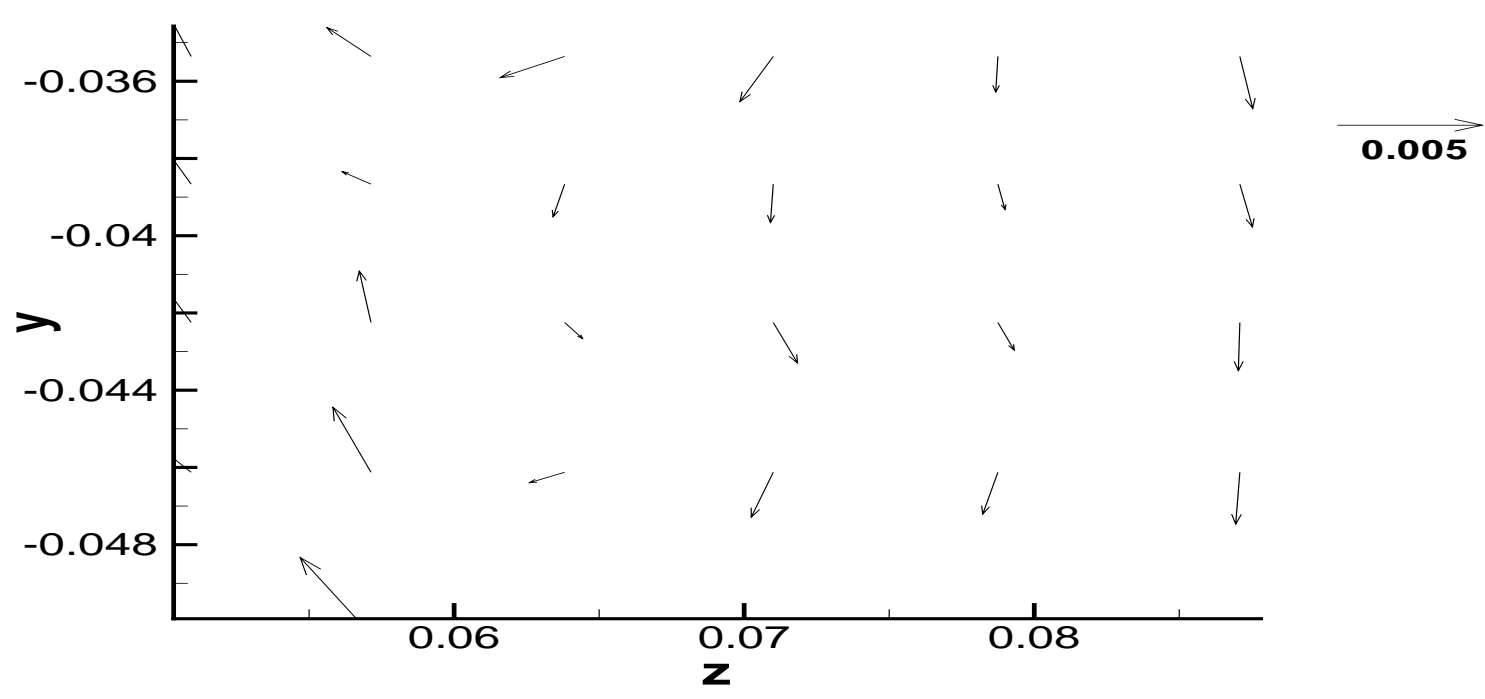

(b) The enlarged view of area A in Fig.7.23(a)

Figure 7.23: Typical instantaneous velocity vectors and the enlargement of area $\mathrm{A}$ on $\mathrm{y}-\mathrm{z}$ plane at $\mathrm{x} / \mathrm{L}=1.2$ in the ship wake 


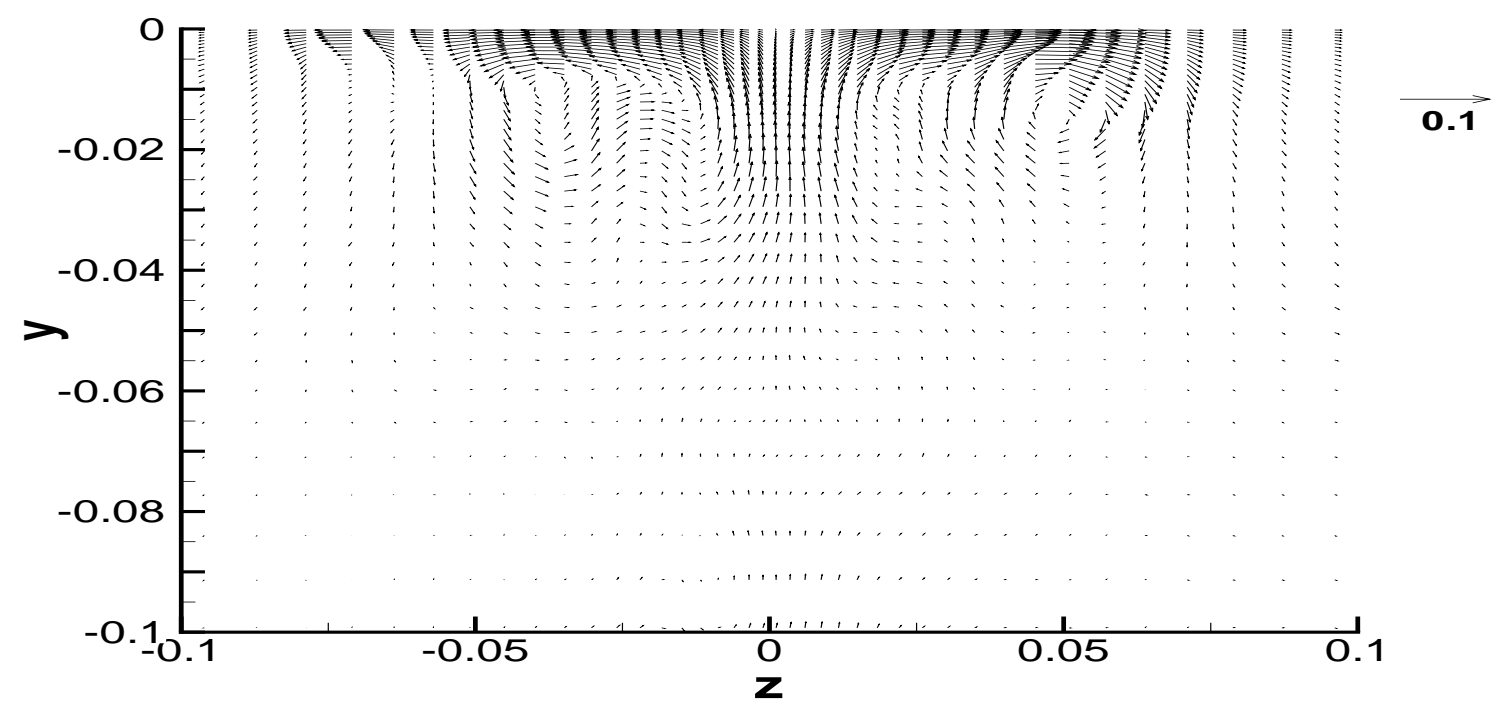

Figure 7.24: Typical instantaneous velocity vectors on $y-z$ plane at $x / L=1.4$ in the ship wake

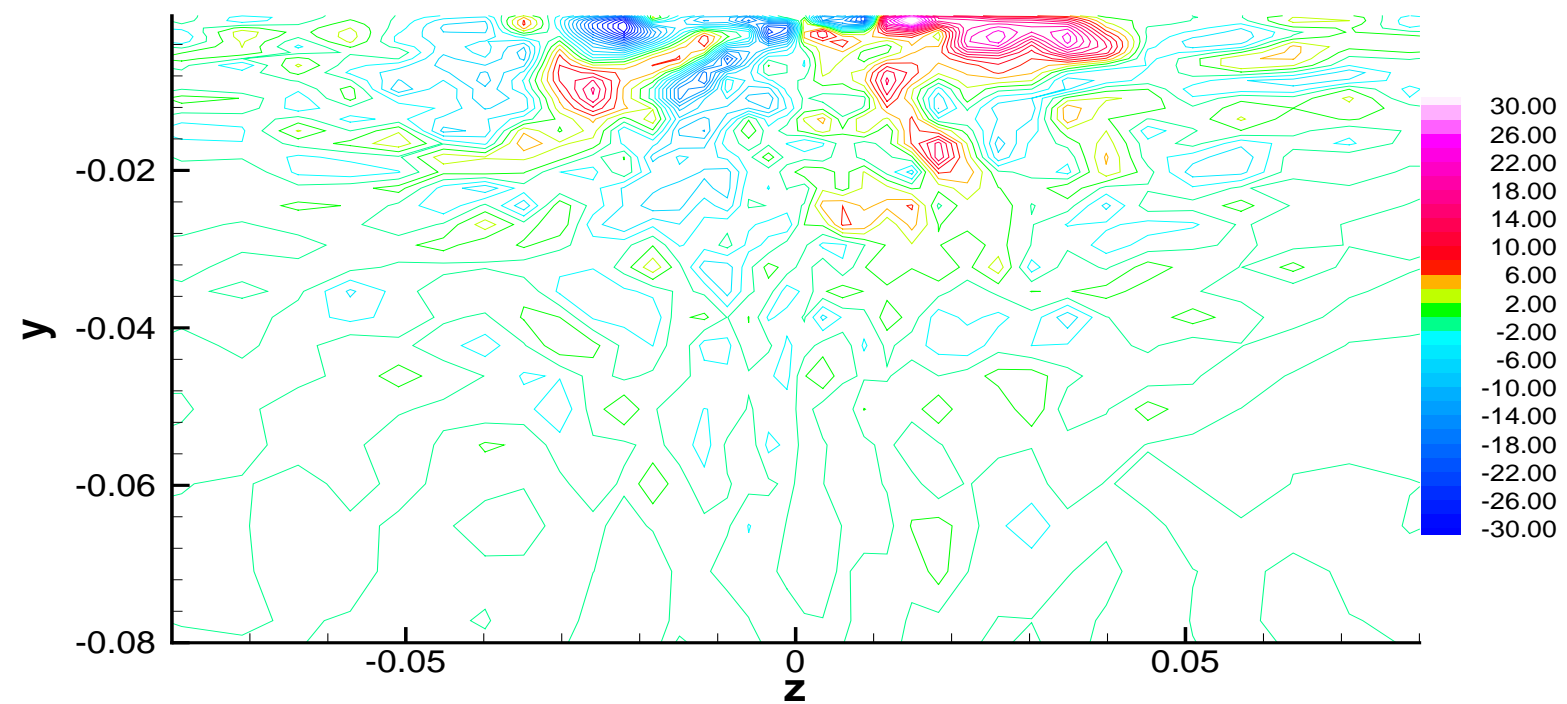

Figure 7.25: Typical instantaneous vorticity contours $\left(\omega_{x}\right)$ on $y-z$ plane at $x / L=0.2$ in the ship wake 


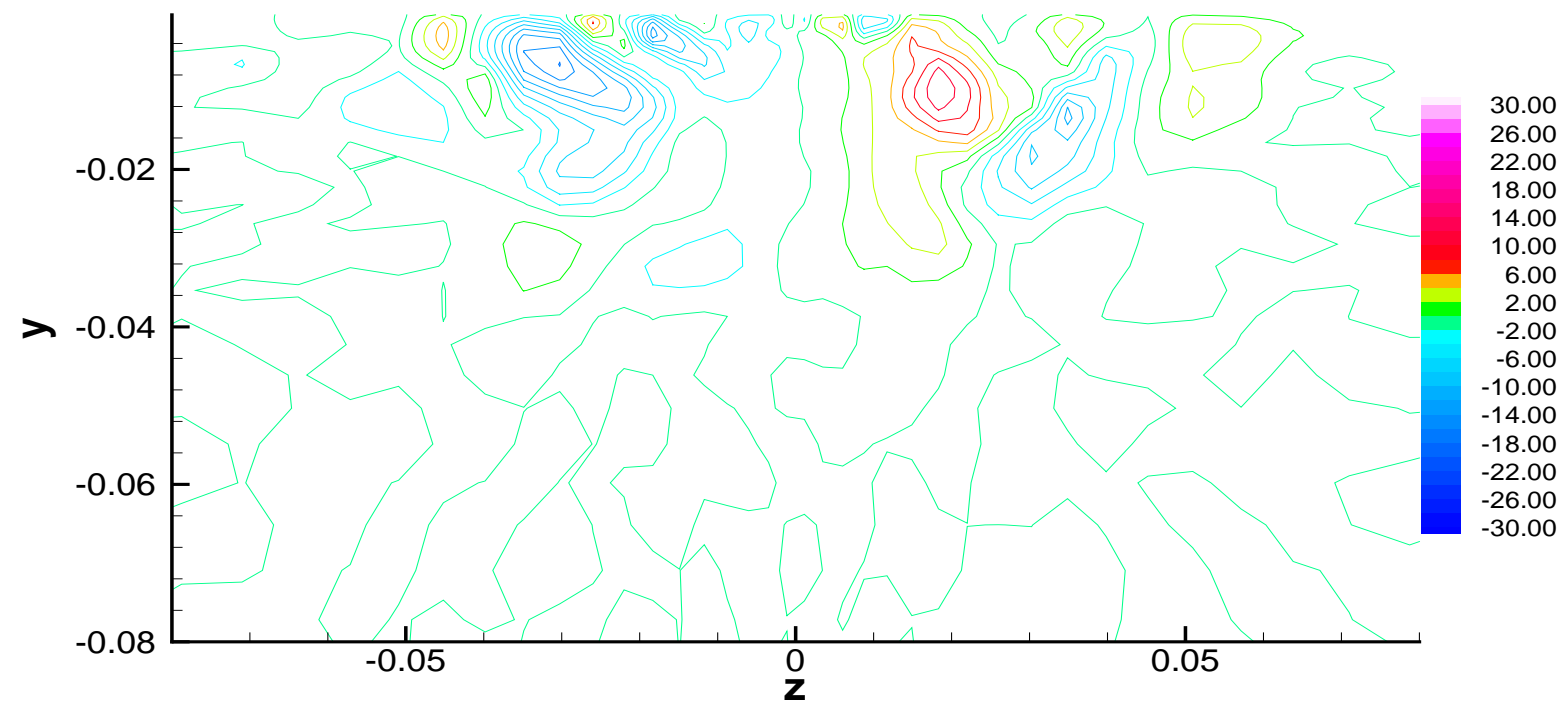

Figure 7.26: Typical instantaneous vorticity contours $\left(\omega_{x}\right)$ on $y-z$ plane at $x / L=0.6$ in the ship wake

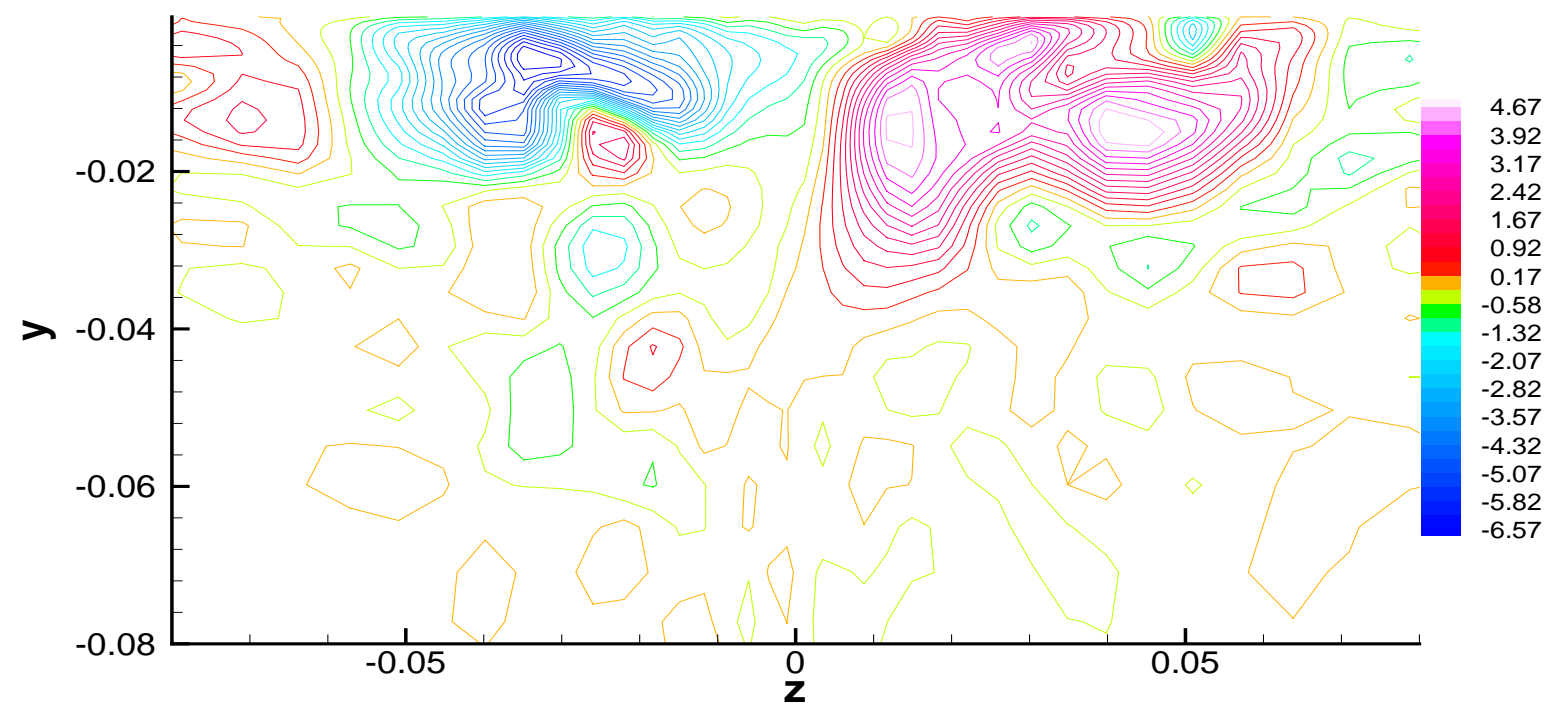

Figure 7.27: Typical instantaneous vorticity contours $\left(\omega_{x}\right)$ on $y-z$ plane at $x / L=1.2$ in the ship wake 


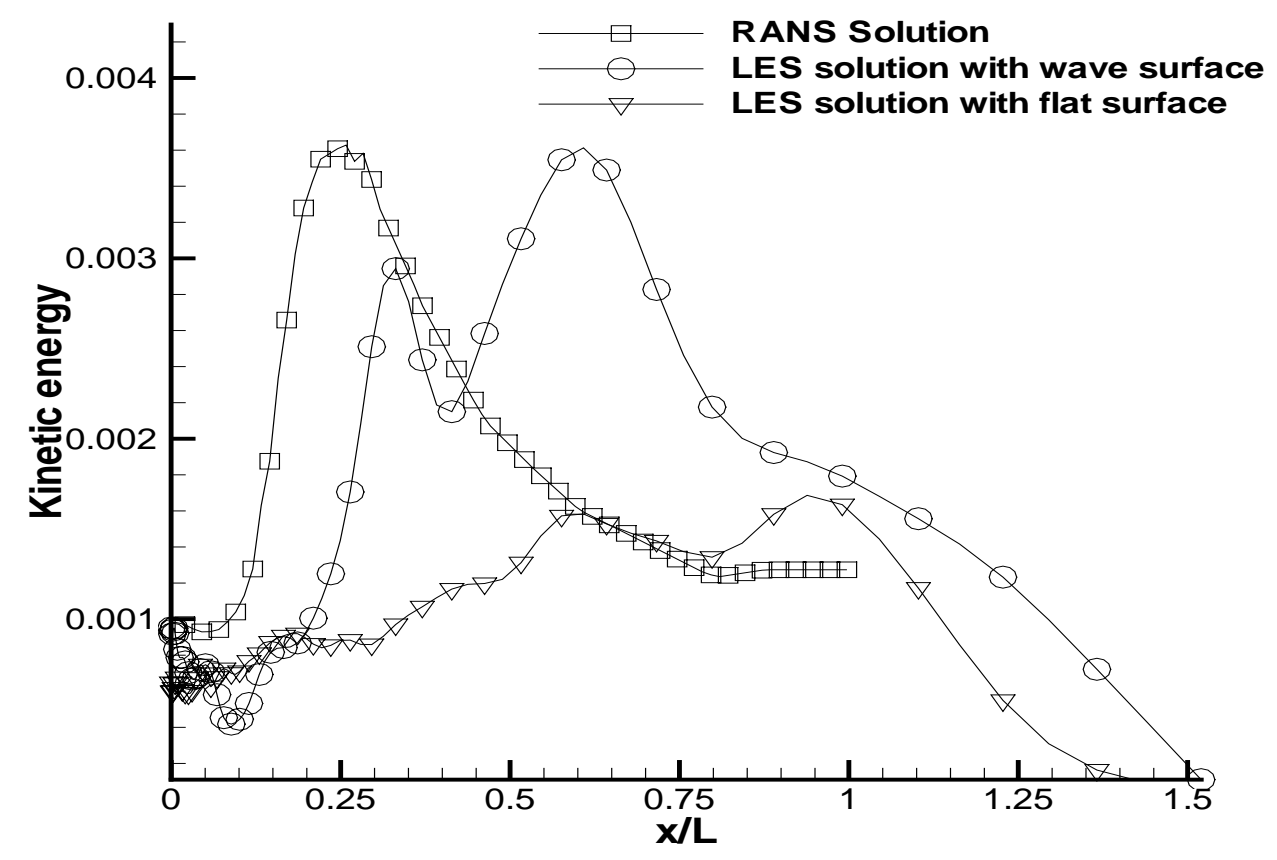

Figure 7.28: Resolved turbulent kinetic energy at $\mathrm{Y} / \mathrm{L}=-0.02$ 


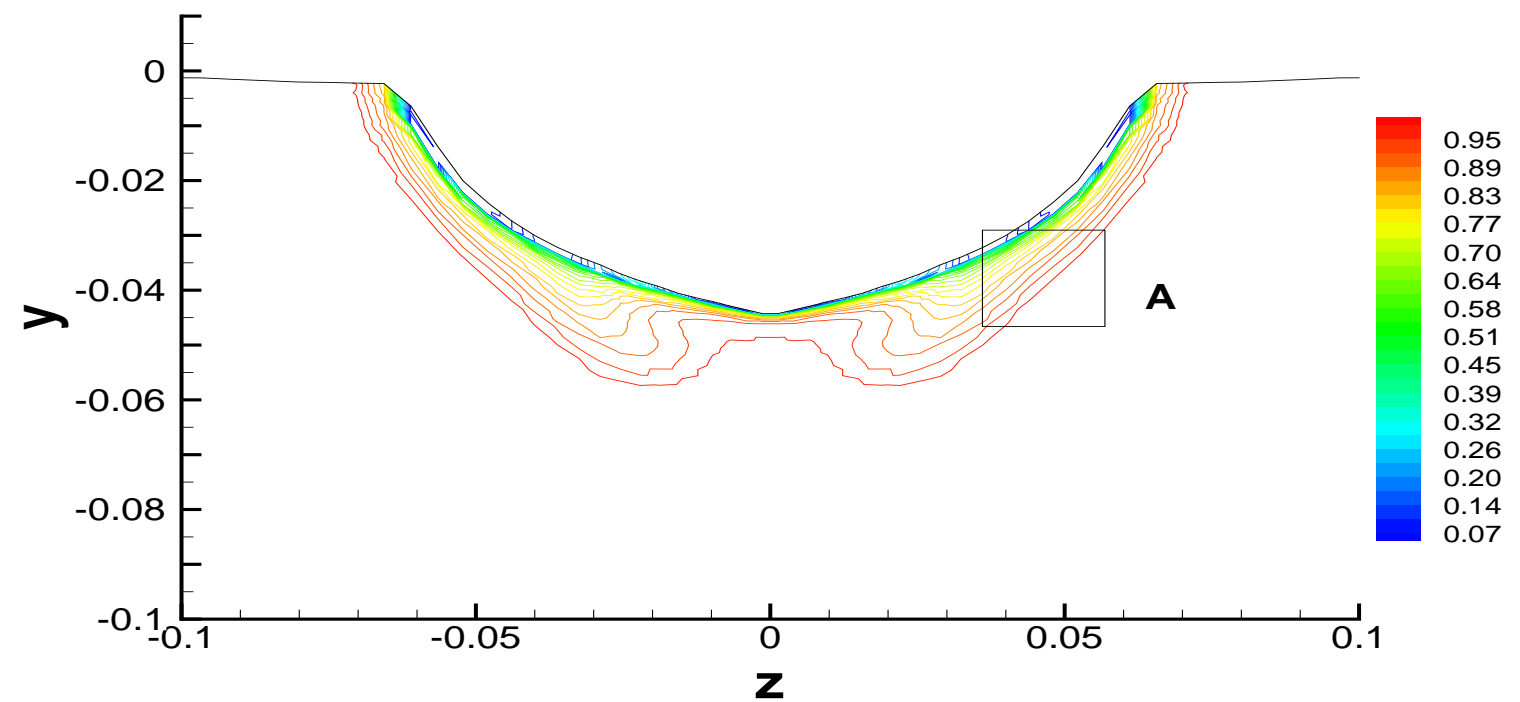

Figure 7.29: The mean streamwise velocity at the inlet plane for case 3

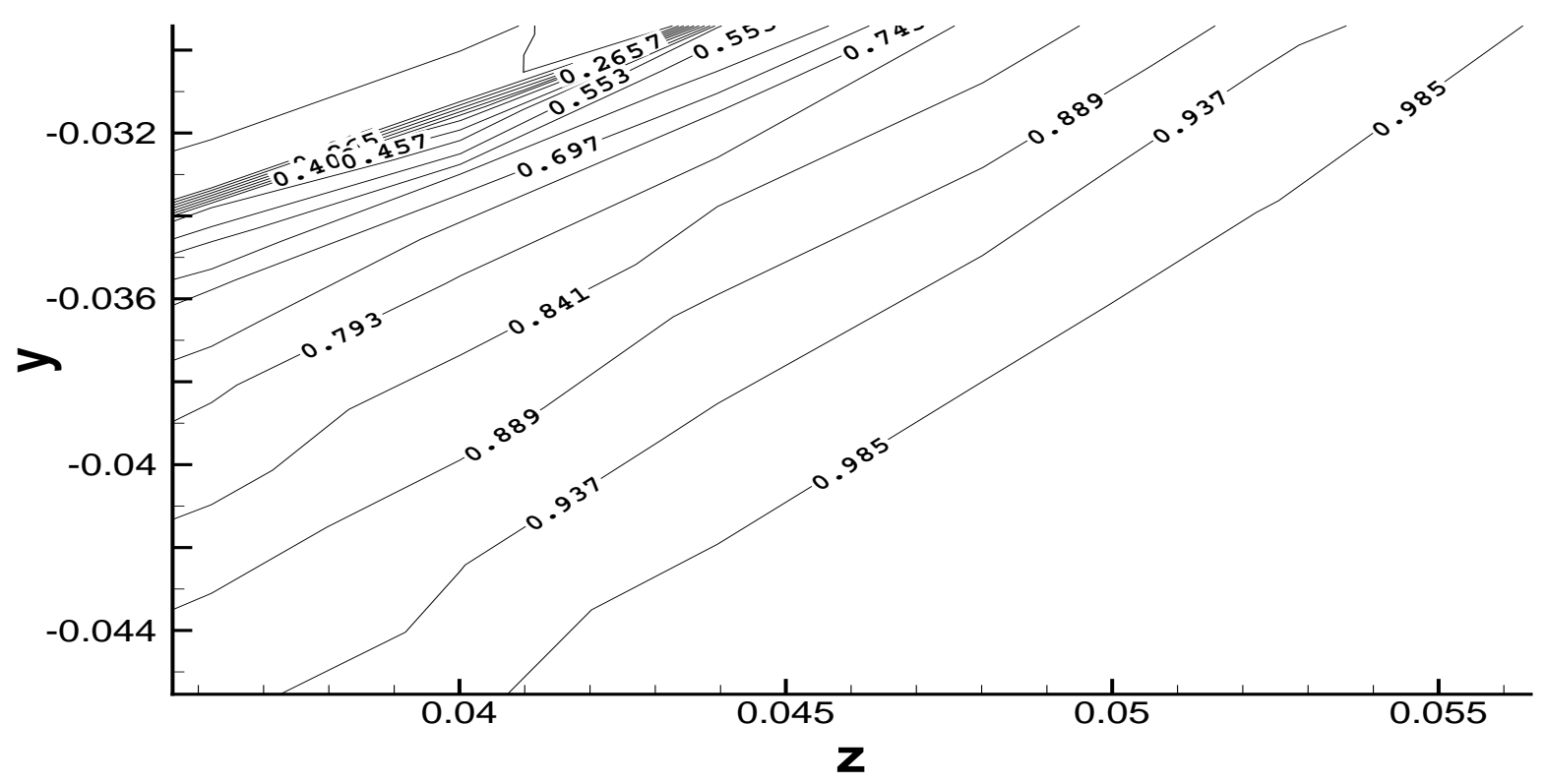

Figure 7.30: The detail information of area $\mathbf{A}$ in Fig. 7.29 


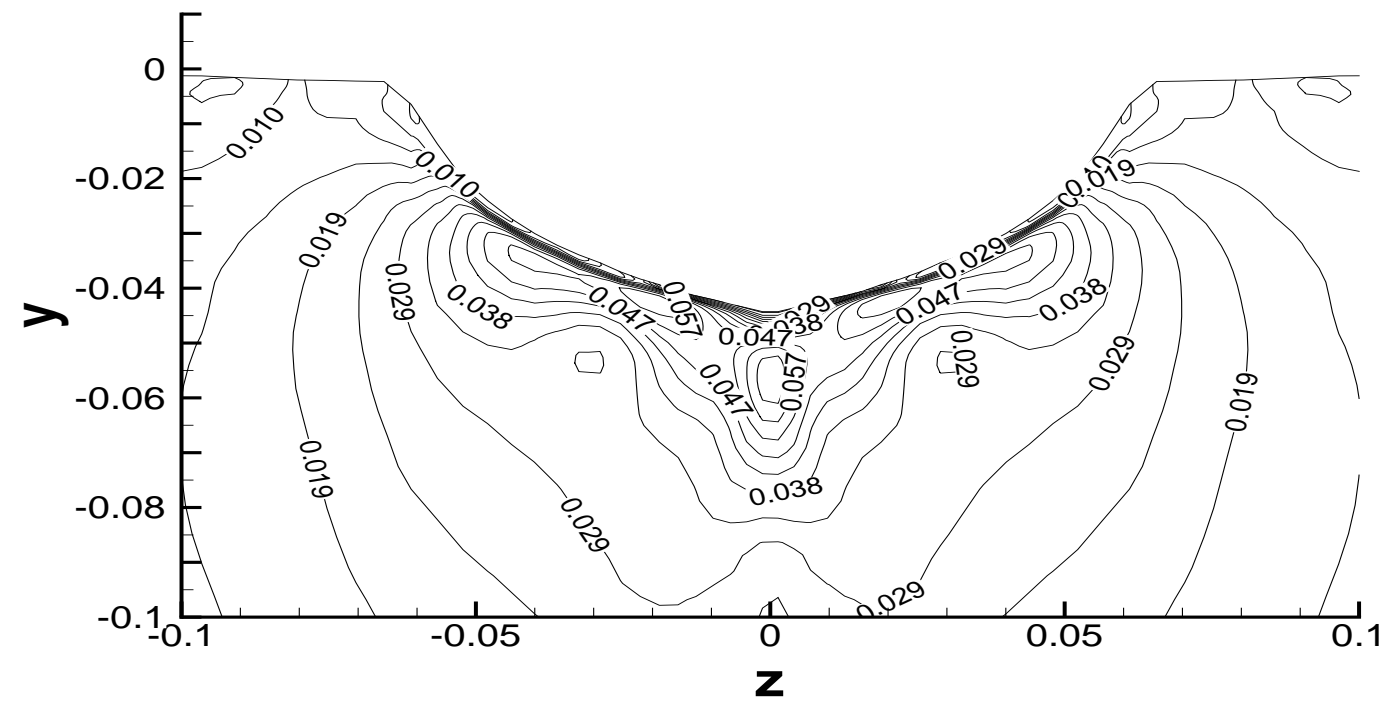

Figure 7.31: The mean vertical velocity at the inlet plane for case 2

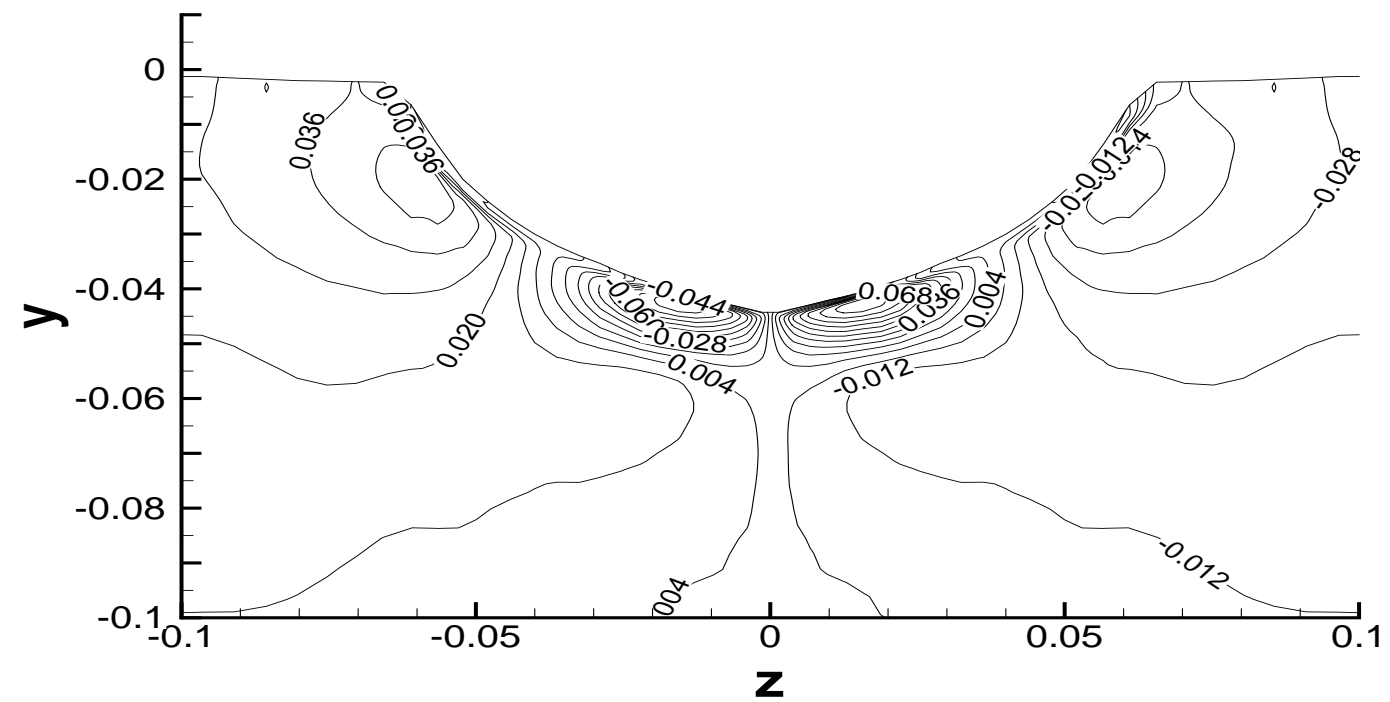

Figure 7.32: The mean spanwise velocity at the inlet plane for case 3 


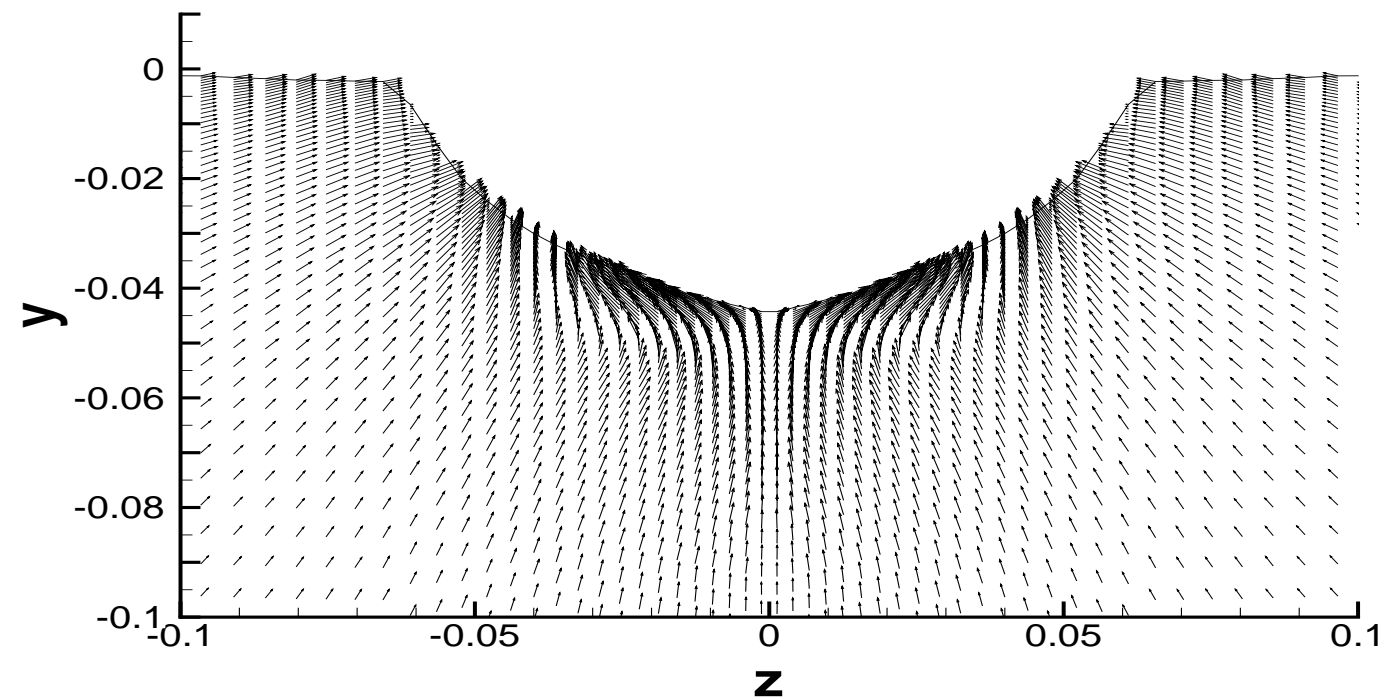

$\overrightarrow{0.1}$

Figure 7.33: The mean velocity vectors at the inlet plane for case 3

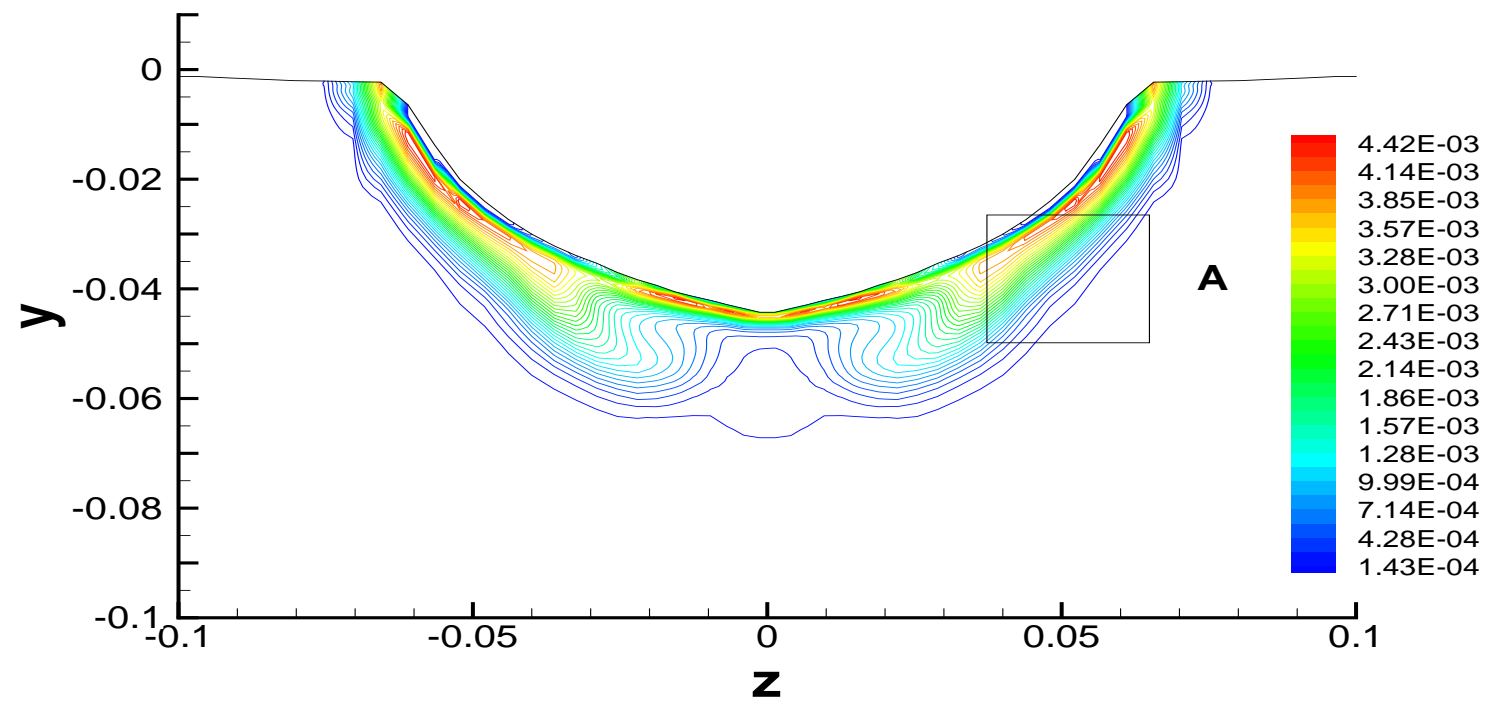

Figure 7.34: The turbulent kinetic energy at the inlet plane for case 3 


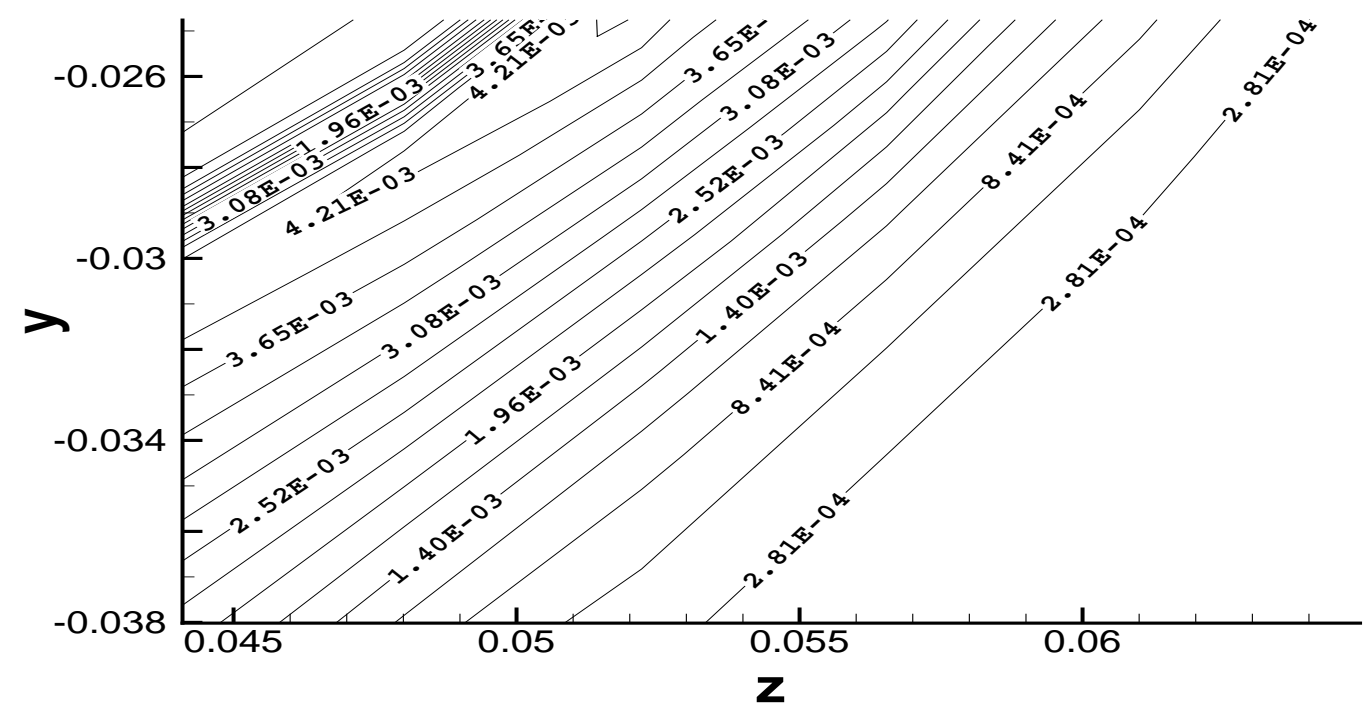

Figure 7.35: The detail information of area $\mathbf{A}$ in Fig. $\mathbf{7 . 3 4}$

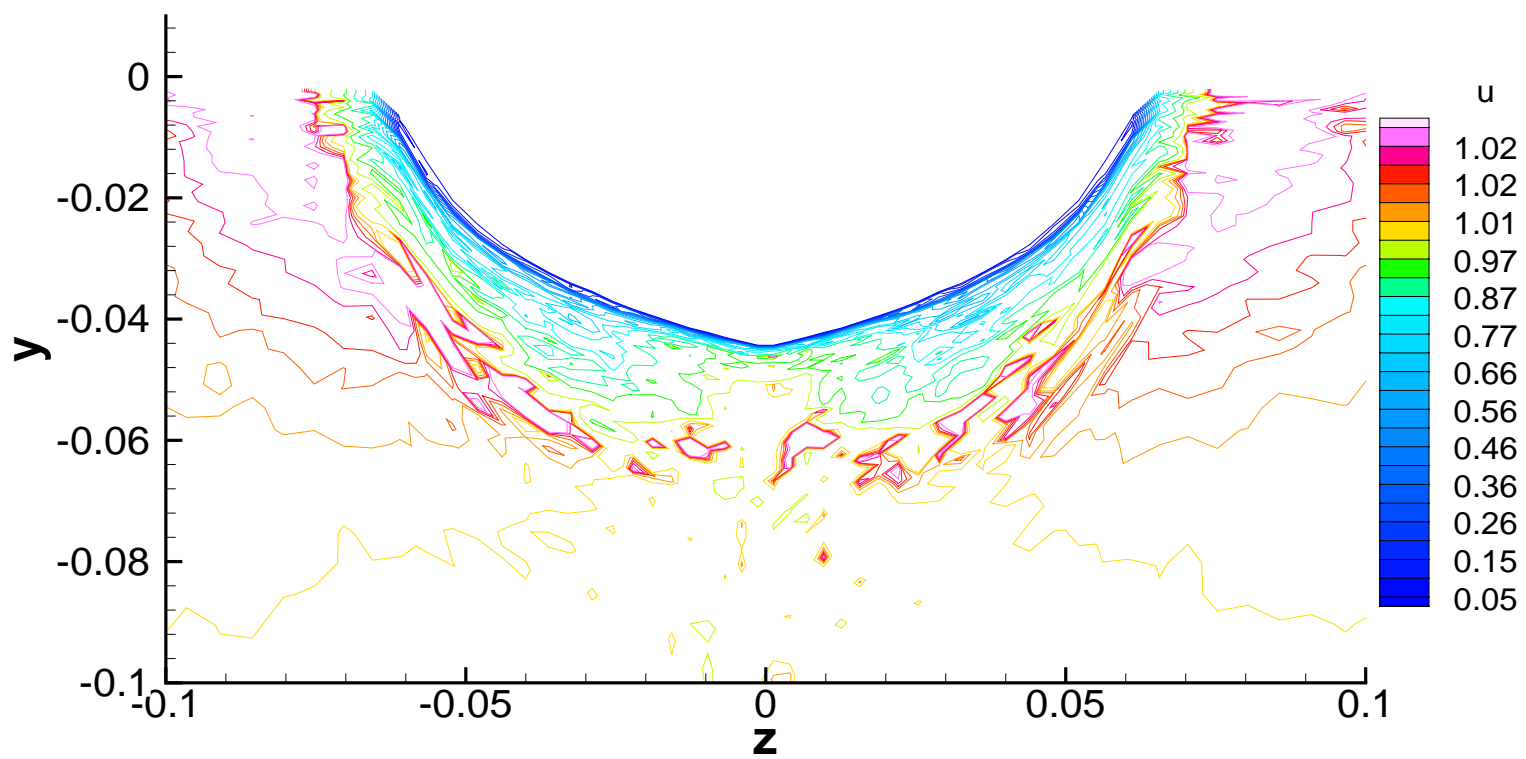

Figure 7.36: The instantaneous streamwise velocity at the inlet plane for case 3 


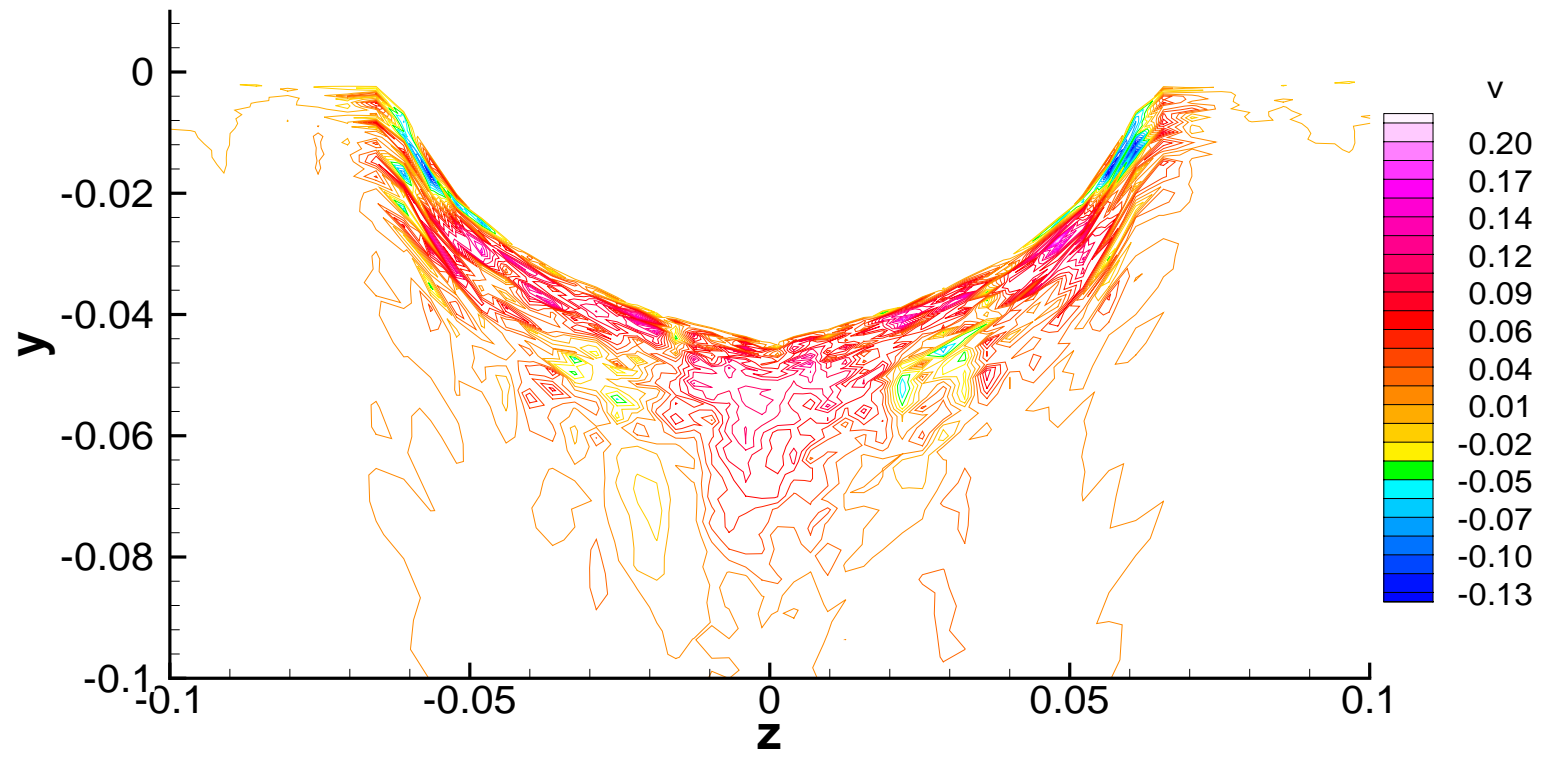

Figure 7.37: The instantaneous vertical velocity at the inlet plane for case 3

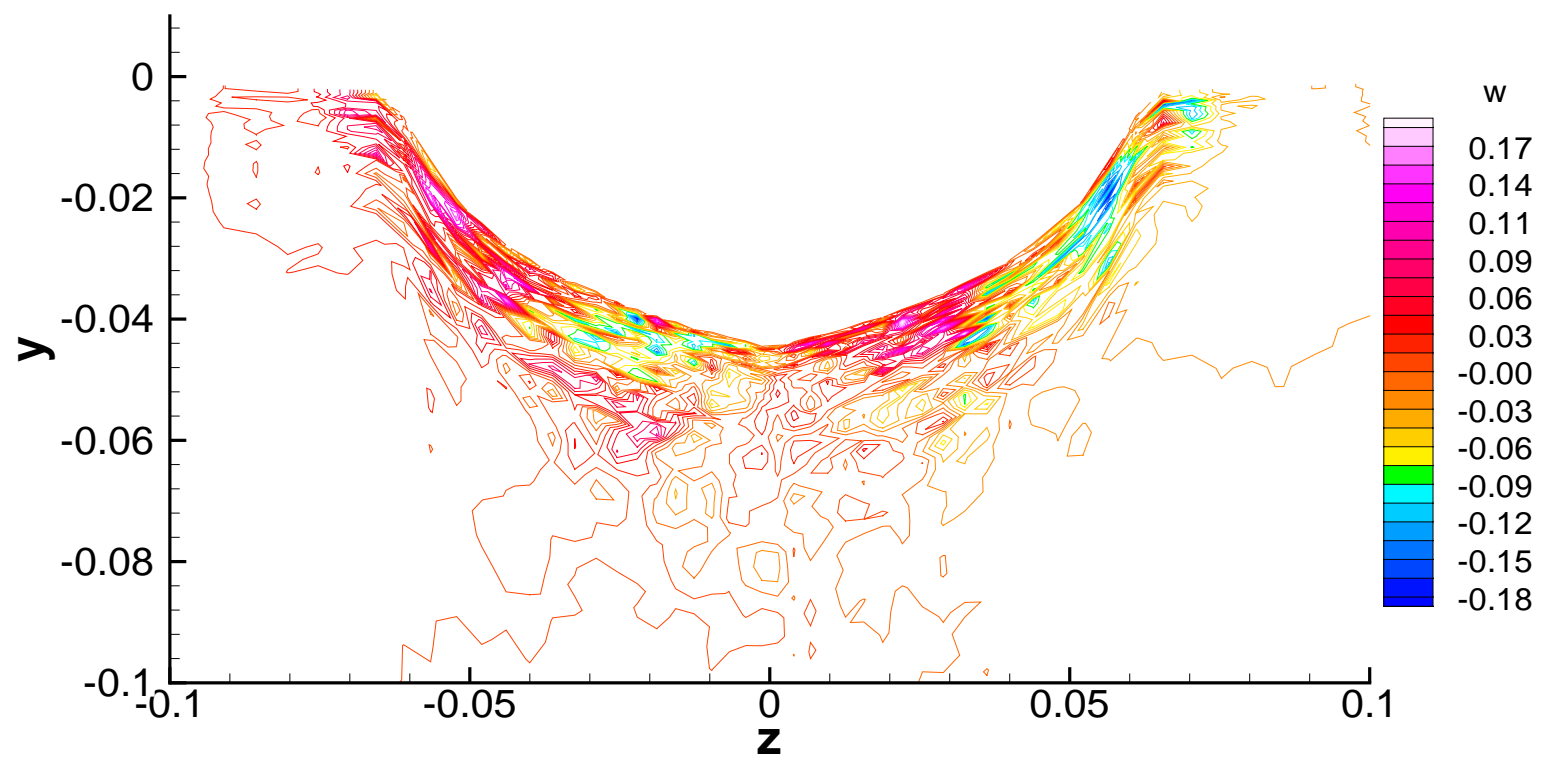

Figure 7.38: The instantaneous spanwise velocity at the inlet plane for case 3 


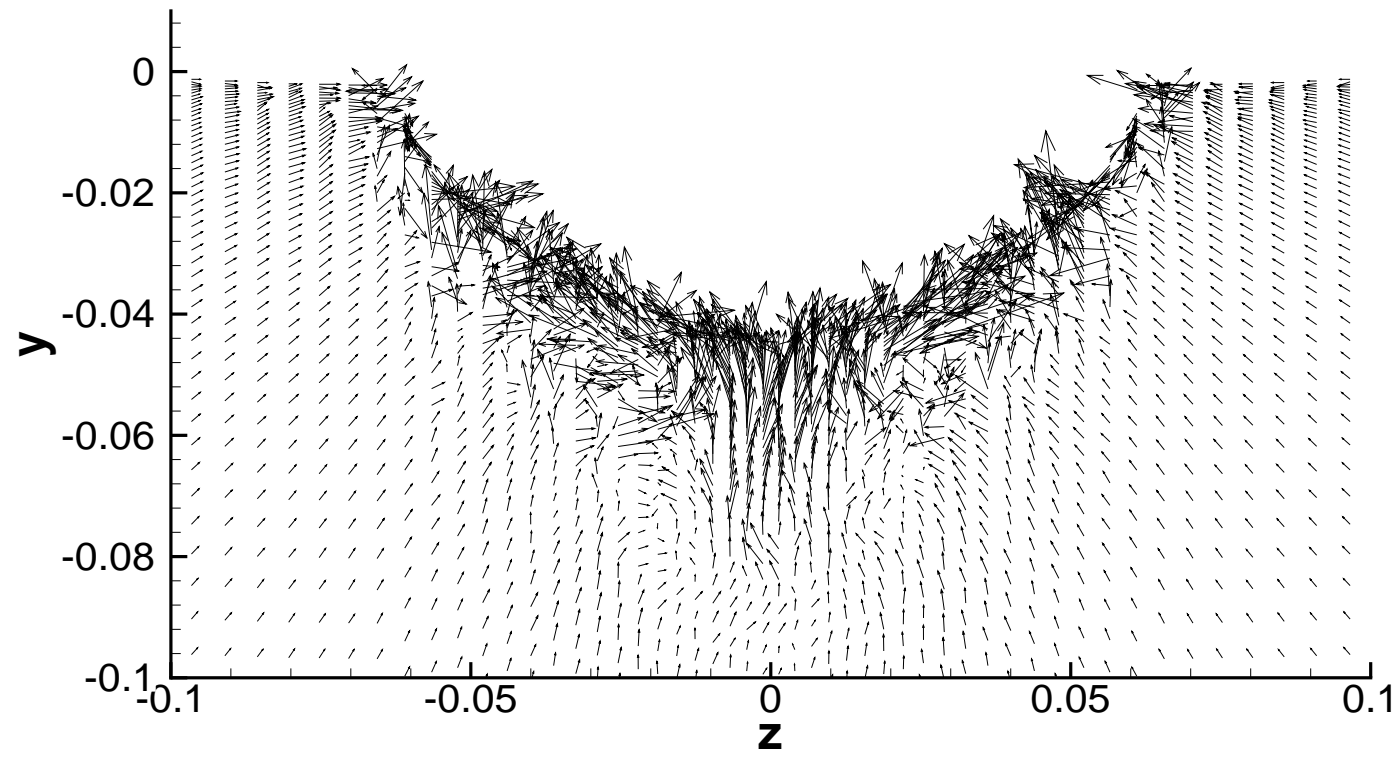

0.1

Figure 7.39: The instantaneous velocity vectors at the inlet plane for case 3

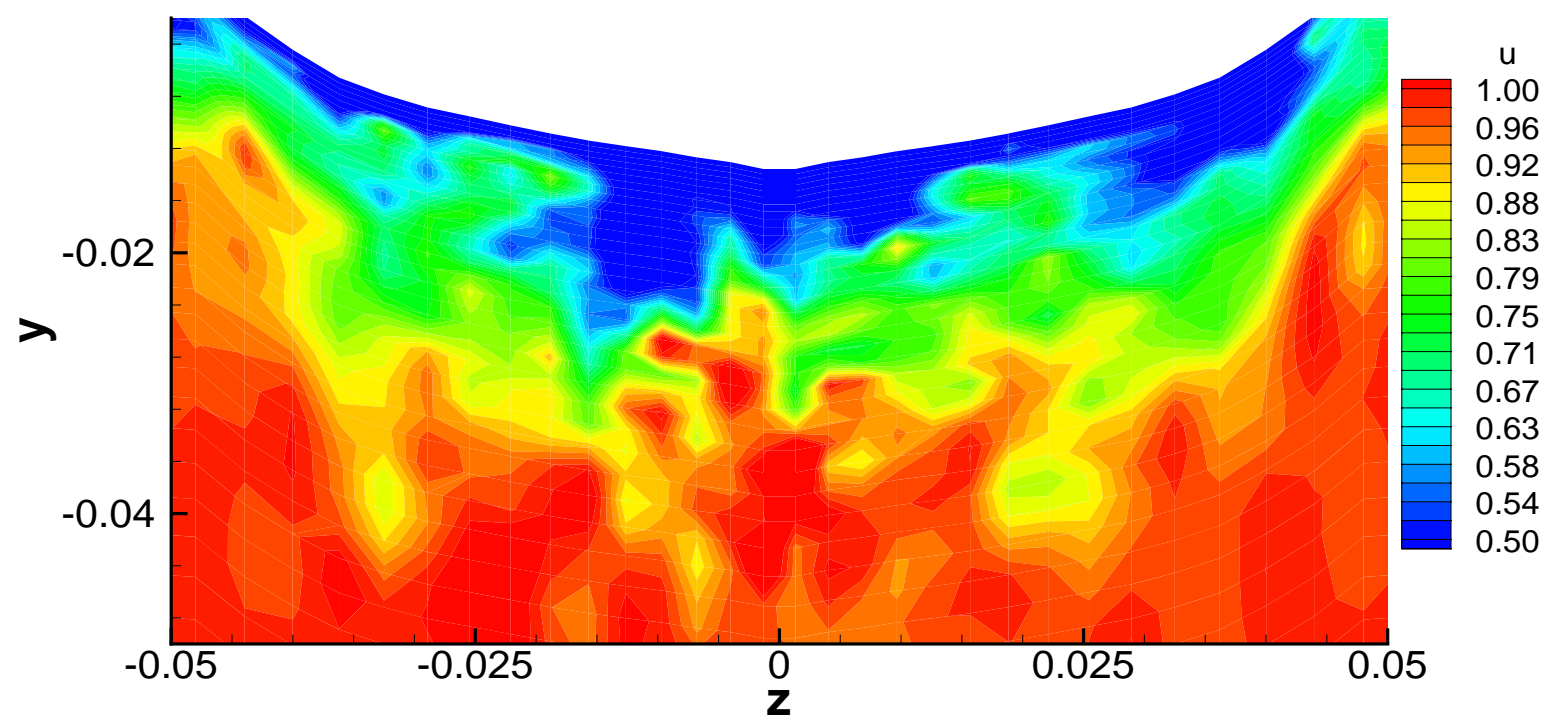

Figure 7.40: The instantaneous streamwise velocity at $x / L=0.935$ for case 3 


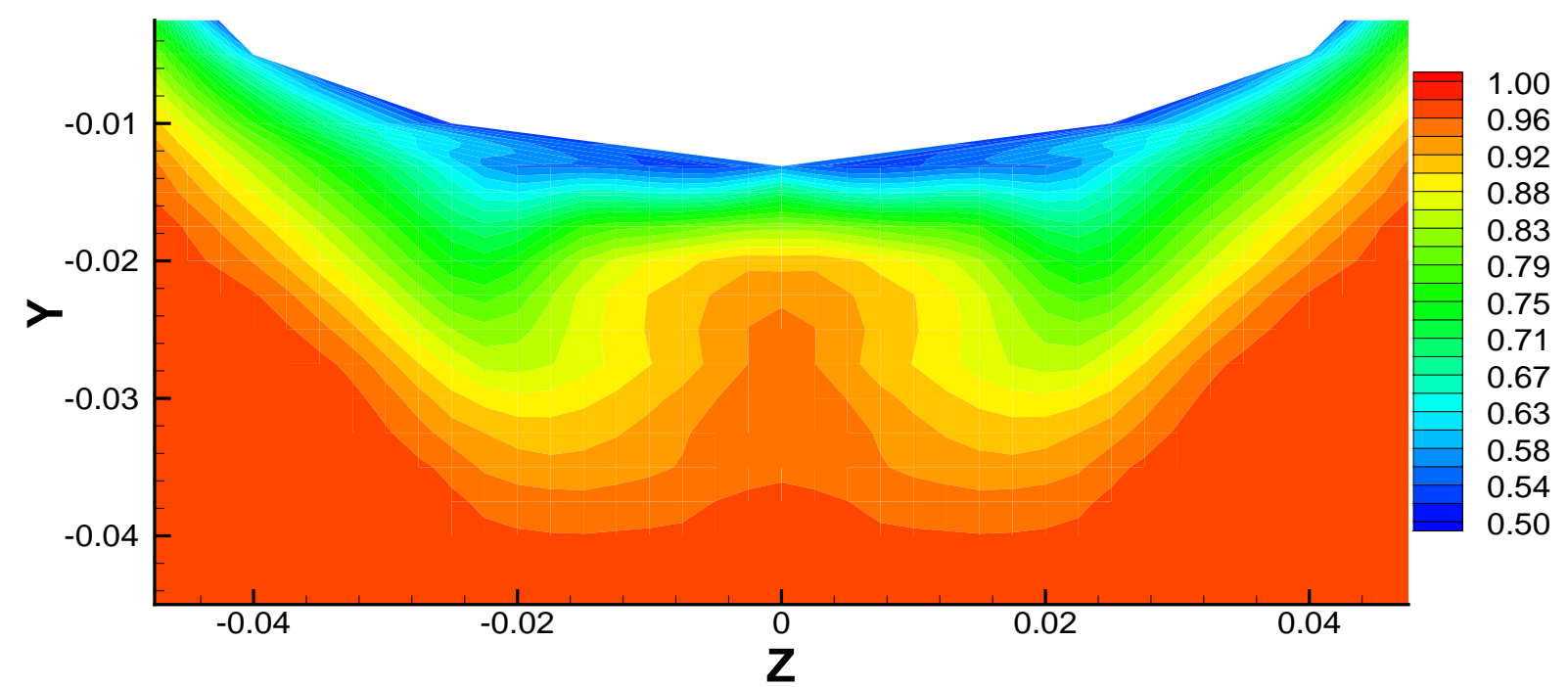

Figure 7.41: The mean streamwise velocity at $x / L=0.935$ from measurements (Stern and Longo, 2001)

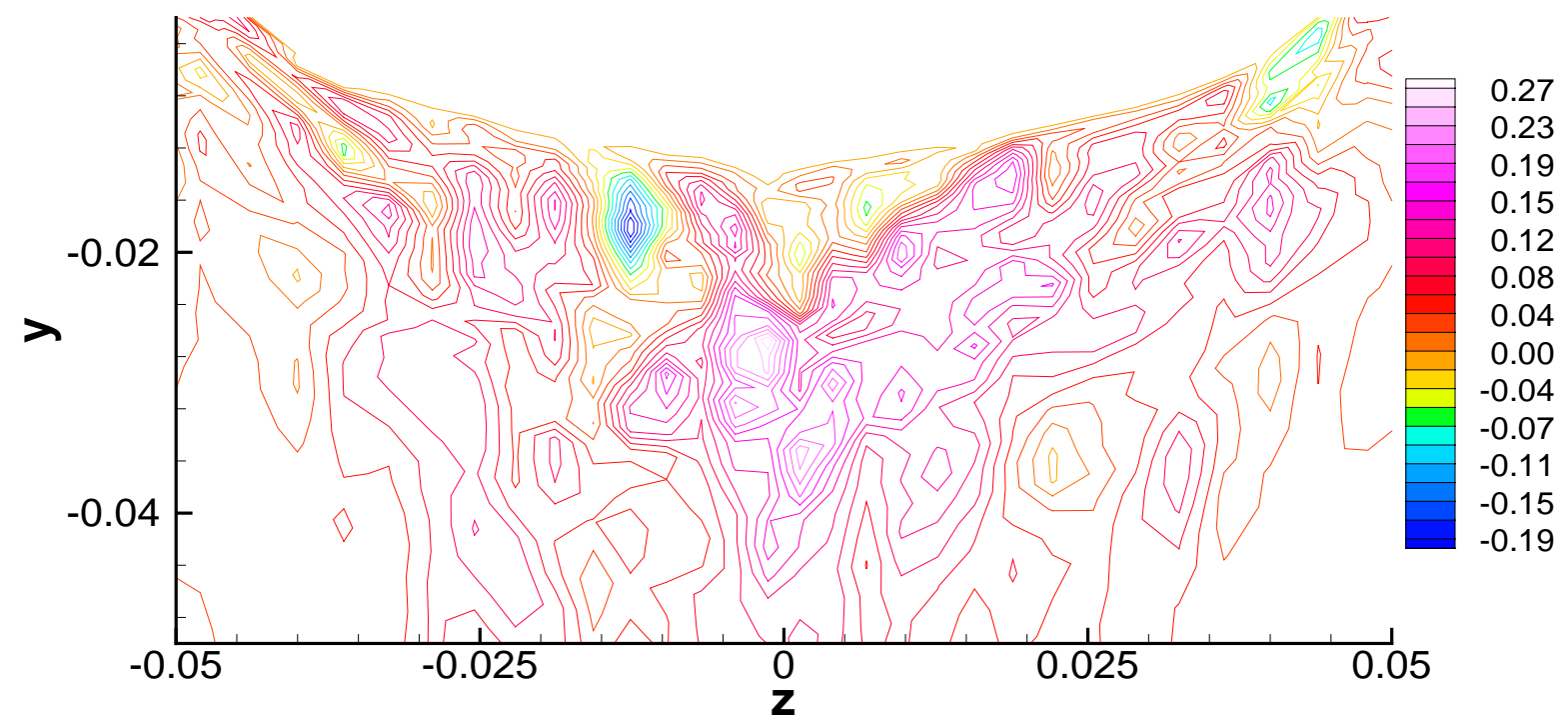

Figure 7.42: The instantaneous vertical velocity at $x / L=0.935$ for case 3 


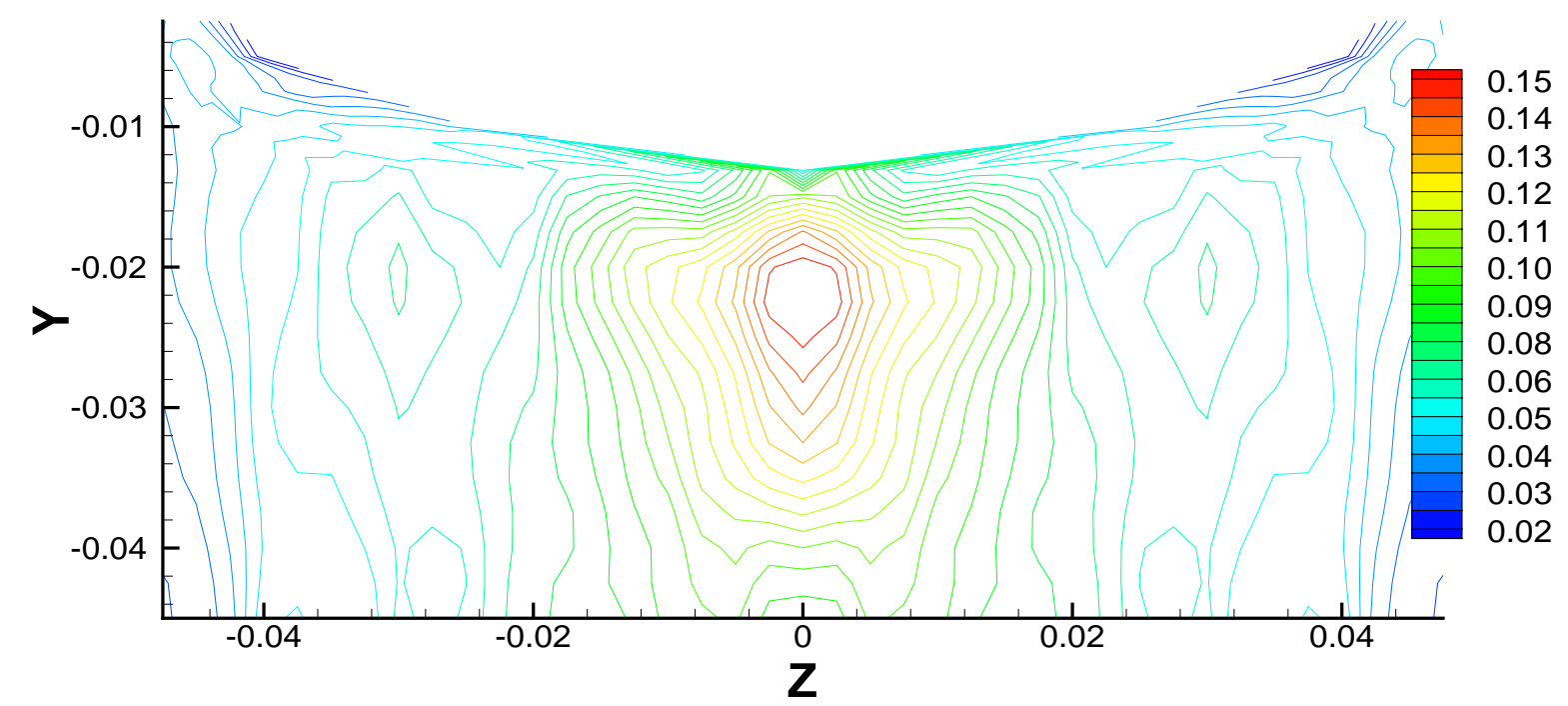

Figure 7.43: The mean vertical velocity at $x / L=0.935$ from measurements (Stern and Longo, 2001) 


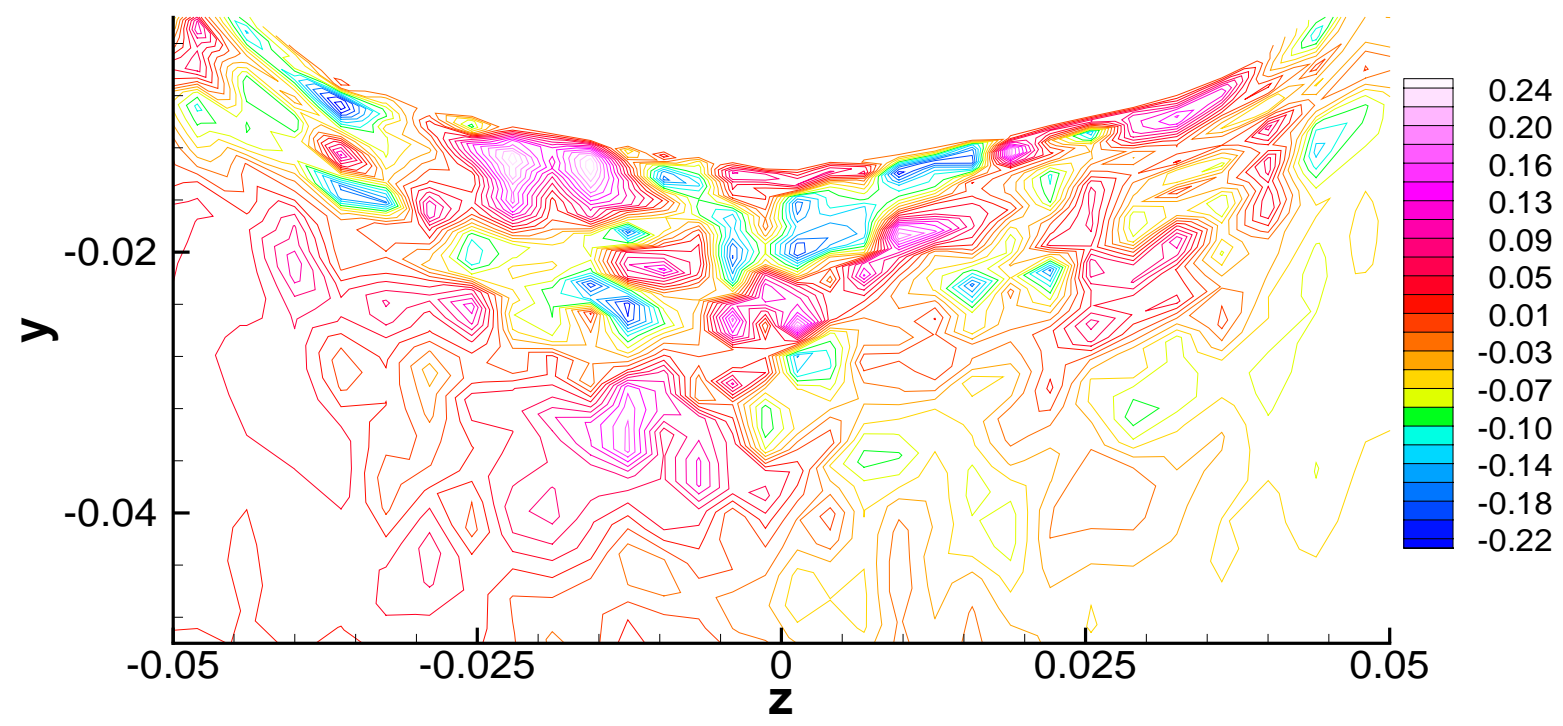

Figure 7.44: The instantaneous spanwise velocity at $x / L=0.935$ for case 3

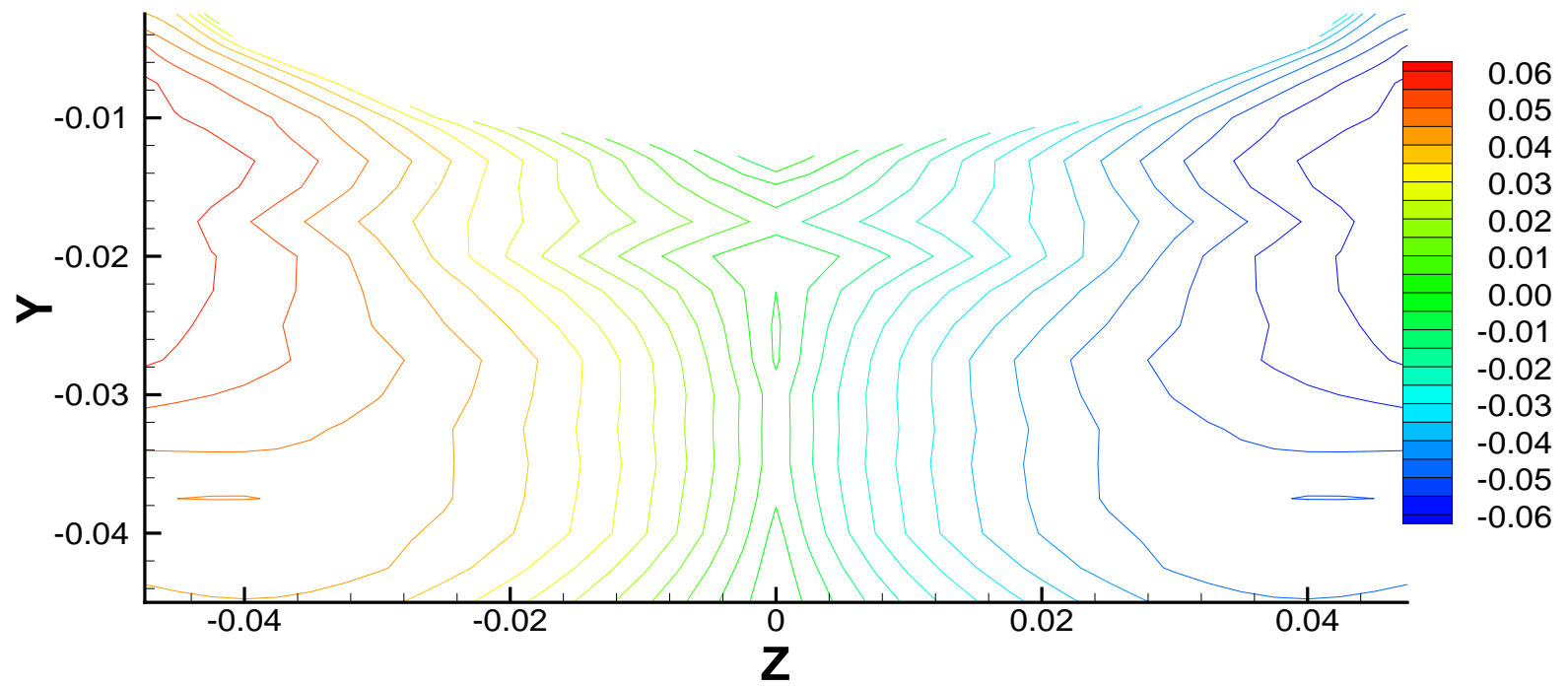

Figure 7.45: The mean spanwise velocity at $x / L=0.935$ from measurements (Stern and Longo, 2001) 


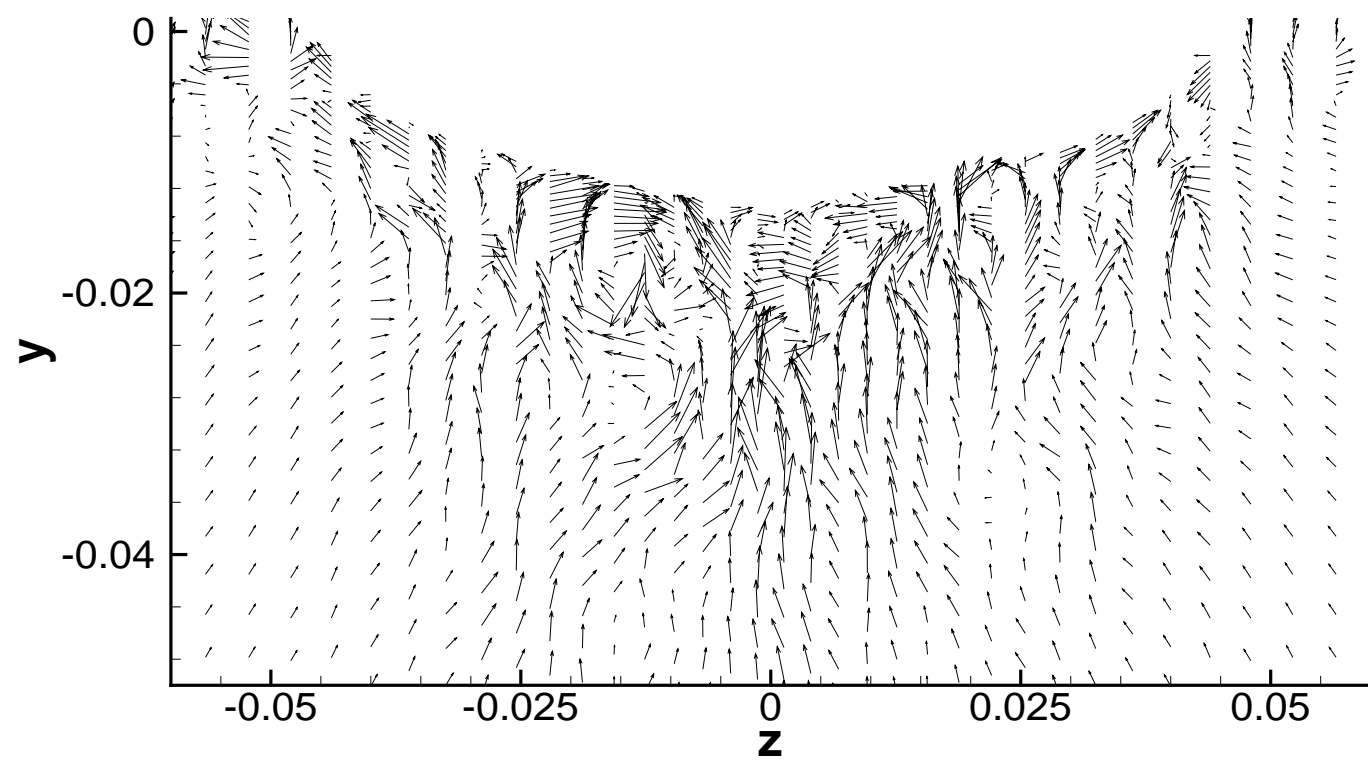

0.1

Figure 7.46: The instantaneous velocity vectors at $x / L=0.935$ for case 3

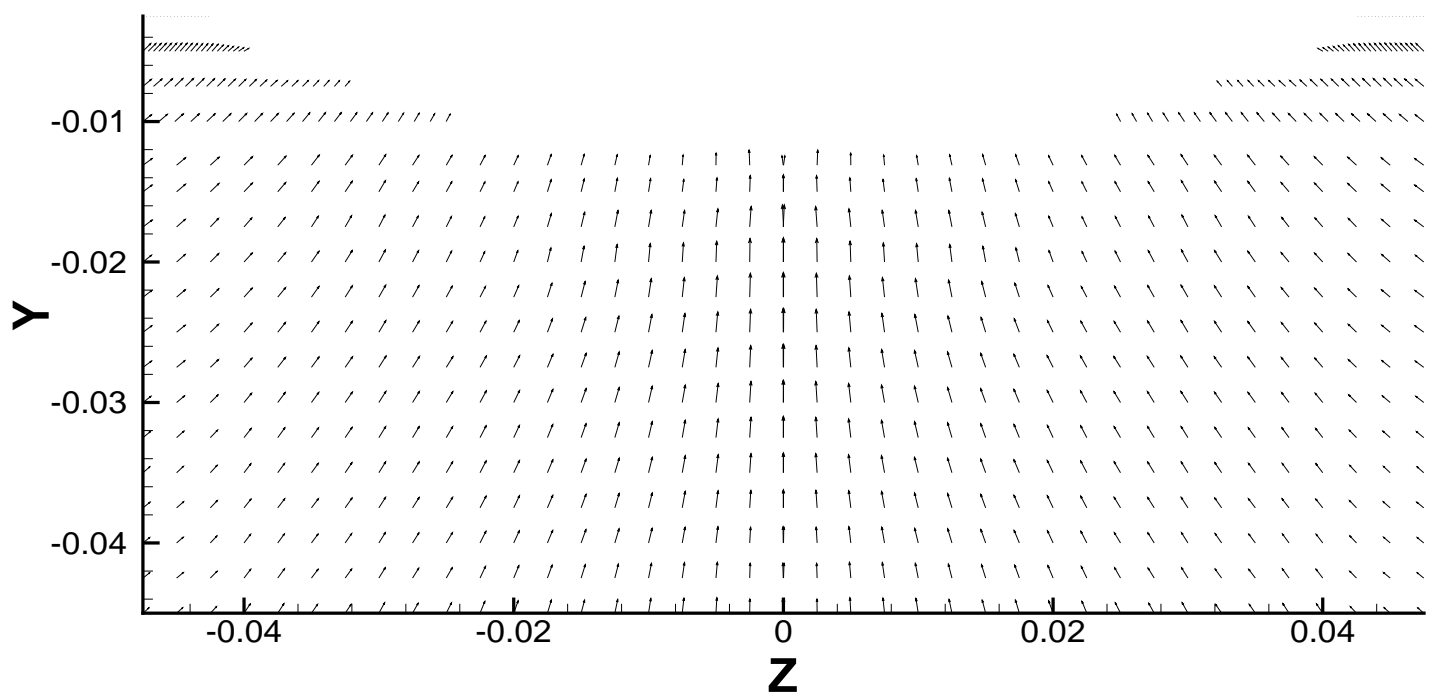

Figure 7.47: The mean velocity vectors at $x / L=0.935$ from measurements (Stern and Longo, 2001) 


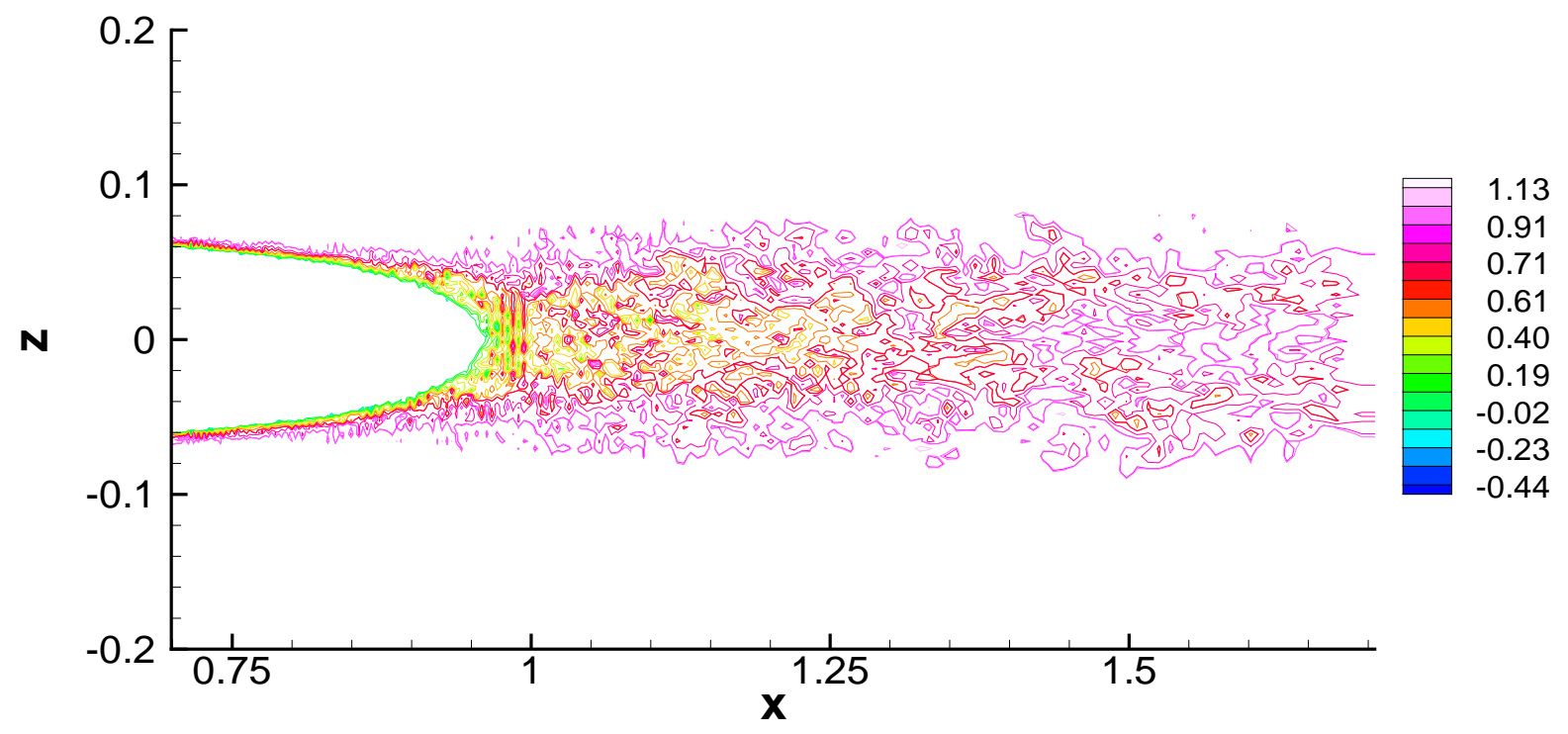

Figure 7.48: The instantaneous streamwise velocity at the free surface for case 3

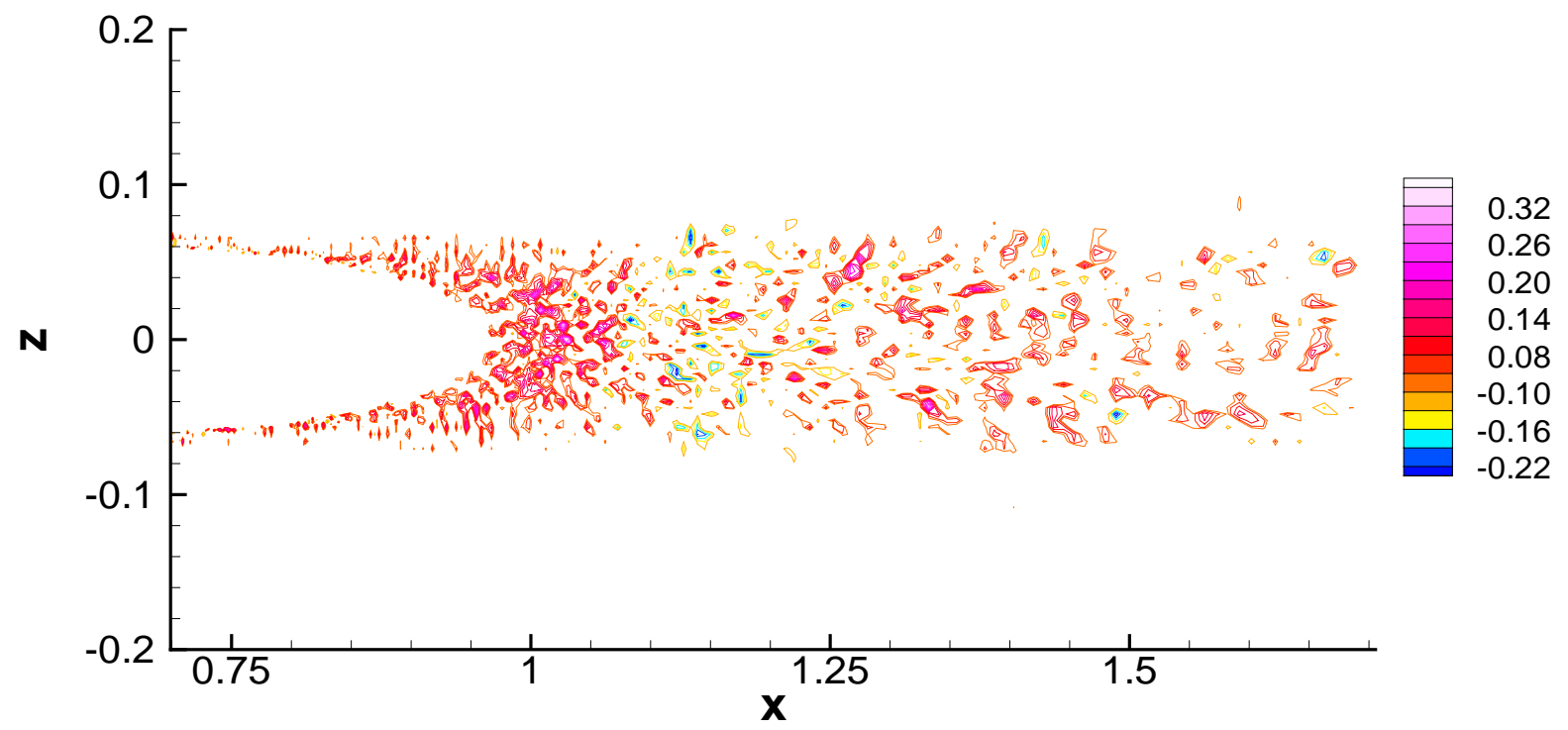

Figure 7.49: The instantaneous vertical velocity at the free surface for case 3 


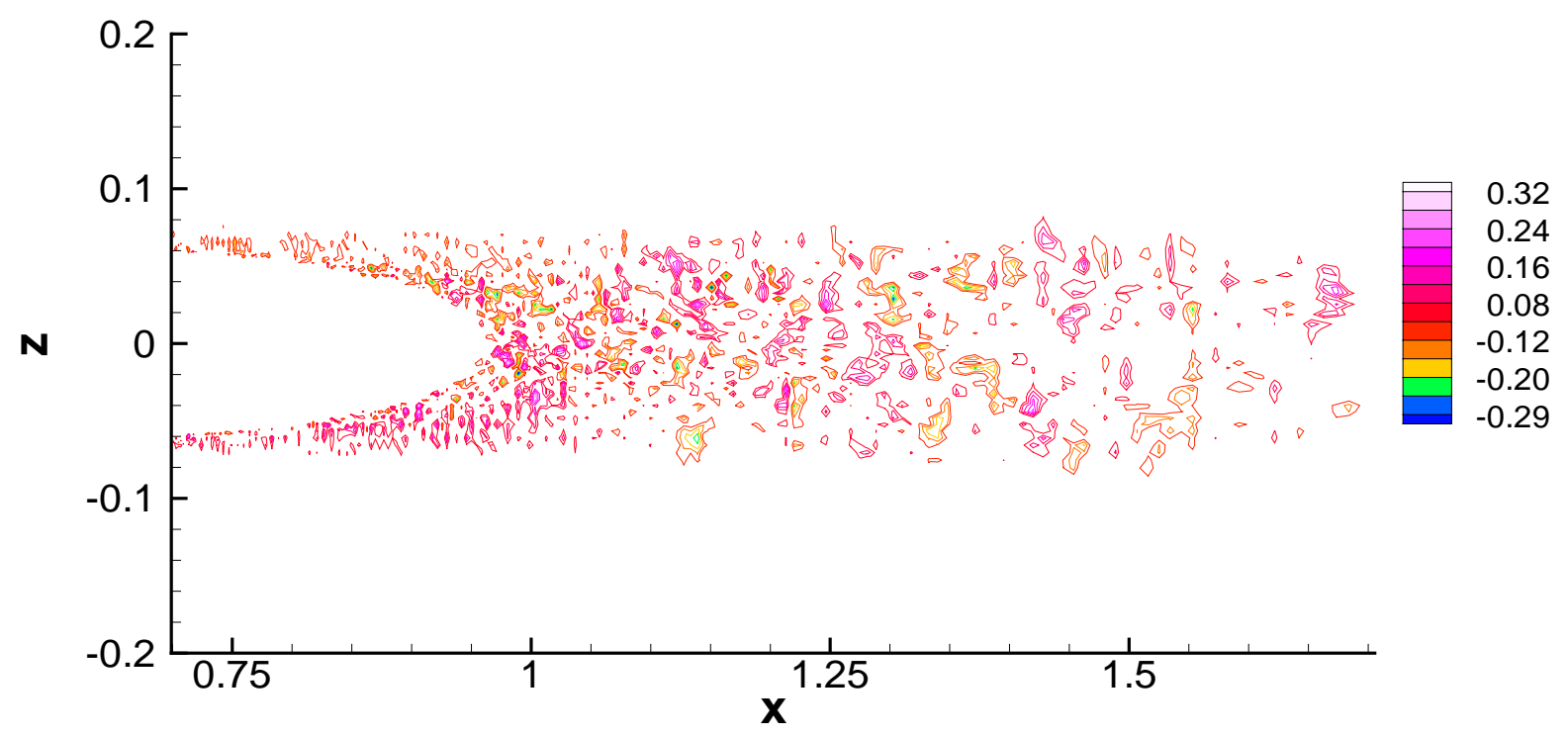

Figure 7.50: The instantaneous spanwise velocity at the free surface for case 3

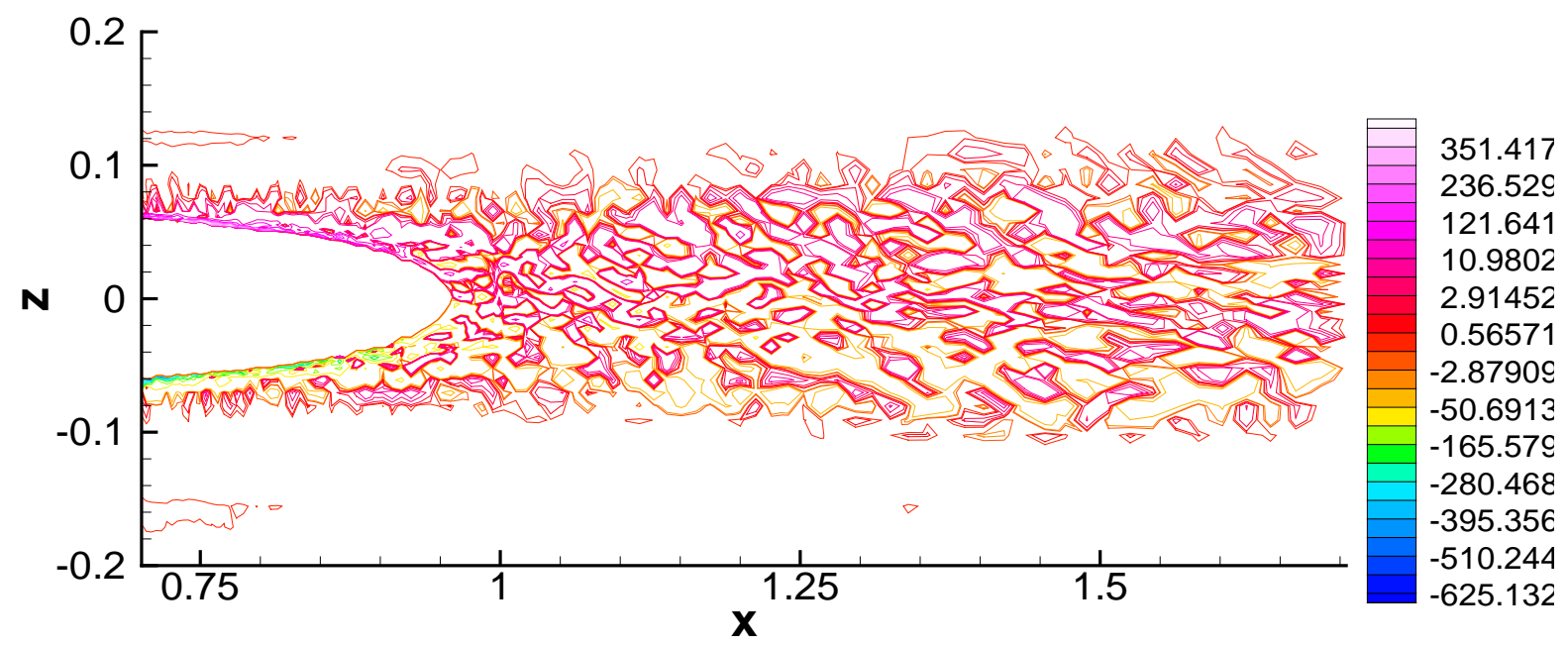

Figure 7.51: The instantaneous spanwise velocity at the free surface for case 3 


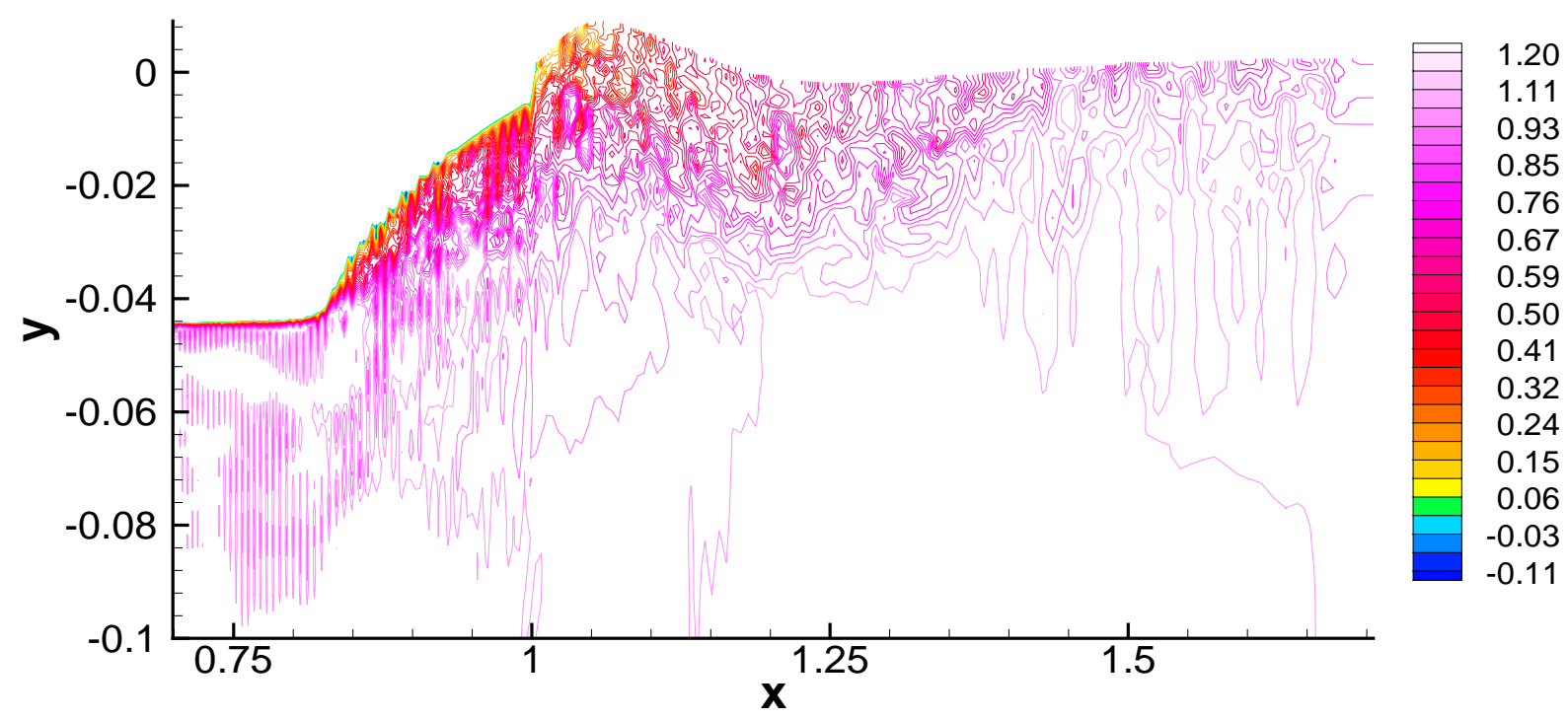

Figure 7.52: The instantaneous streamwise velocity at the center vertical plane for case 3

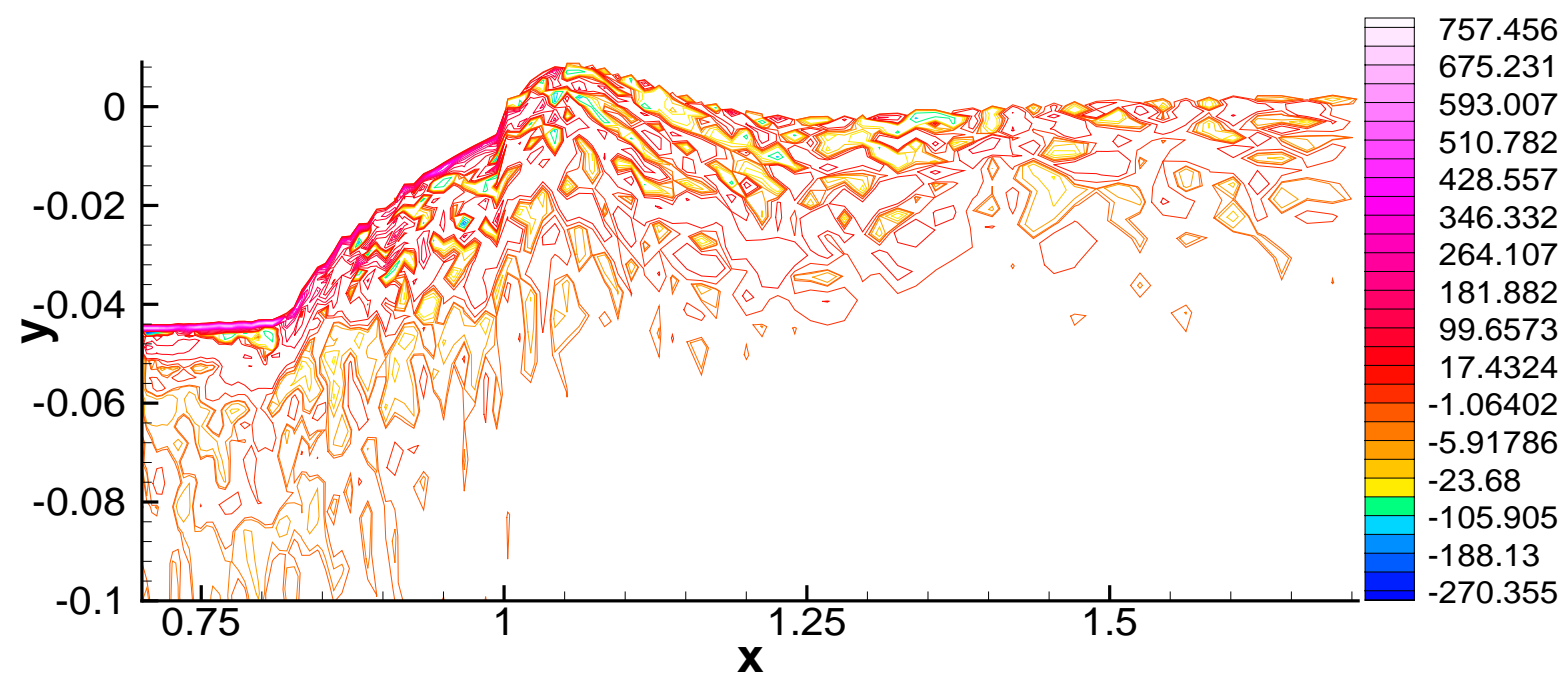

Figure 7.53: The instantaneous spanwise vorticity at the center vertical plane for case 3 


\section{Chapter 8}

\section{Conclusions and Recommendations}

\subsection{Conclusions}

With the fast growth of computer resources, large eddy simulations of high Reynolds number, complex flows are now becoming feasible. To ensure the quality of the LES, efficient, accurate and robust numerical methods as well as effective subgrid-scale models are essential.

Solution methodologies for the time-dependent, three-dimensional, incompressible NavierStokes equations have been verified by applying them to four benchmarks: channel flow, flow past a square cylinder, flow in a shear/mixing layer, and open channel flow. By comparing the simulation results with the experiments, it has been shown that the used solution methodology has favorable accuracy and efficiency.

LES of complex turbulent flows induced by large bluff bodies is still prohibitive due to the requirement of excessively large number of grids to resolve the boundary layer surround the body. In the course of this study a robust approach for reconstructing the instantaneous turbulent flow field from a given mean flow field, namely - the Random Flow Generation (RFG) technique, has been improved and validated. This approach can be used to initialize the turbulent flow field and also to prescribe realistic turbulent inflow boundary conditions for LES. By using this technique, the turbulent flow field can be reproduced with good accuracy, even when limited information is available on the mean flow. This information 
can include the turbulent kinetic energy, its dissipation rate, or shear stresses.

A one equation SGS model has been selected and modified to suit the purpose of this study. This model is based on the subgrid scale turbulent kinetic energy which is solved simultaneously with the Navier-Stokes equations. A near-wall modification aimed at improving the near wall resolution, which includes dynamically adjusting the length scale and improving the dissipation model has been proposed. Energy backscatter, which is an important mechanism in turbulent flows, has been included in the one equation model. The RFG algorithm together with the subgrid scale turbulent kinetic energy and the subgrid length scales were used to generate a subgrid turbulence flow field which was superimposed on the resolved flow field by using two different approaches. Channel flow was used as a test case to validate the proposed techniques. The results show that the one equation model by itself gives the same quality as the well known Smagorinsky model. However, with the backscatter included, the results have been significantly improved.

Before starting the ship wake flow simulation, it was necessary to test the numerical schemes and modeling strategies on a well documented benchmark. For this purpose, the case of flat plate wake flow has been selected. Unlike the used simulation strategies, the flat plate itself has not been included in the calculation domain. Instead, the computational domain starts from a certain distance downstream of the plate's edge. Based on the turbulence parameters, such as the Reynolds stresses and turbulent kinetic energy from measurements, the RFG method was used to produce a time-dependent turbulent inflow boundary. The generated flow field inherits most of the turbulence characteristics of the real flow at this location, with proper turbulence structures, almost exact turbulence intensities, and turbulent shear stresses. Large eddy simulation was then used to solve the wake flow. A good agreement between predictions and the measurements was obtained. The influences of grid resolution, and different SGS models have been studied. With a fine grid, better results were obtained, as should be expected. 
Pursuing the primary objective of simulating the turbulent flow in the near wake of a ship by LES technique, the RFG method was used with inputs from RANS calculation to provide the turbulent inflow boundary. Three different cases have been simulated for the ship model DTMB 5512. The first case was designed to model the pure ship wake (excluding ship hull) with a flat free surface. The large turbulence structures have been well resolved. Two distinct stripes (bilge vortices) develop through the whole wake with apparent turbulence eddies. The resolved turbulent kinetic energy was comparable in magnitude to that of the RANS simulation.

The second test was mainly to estimate the influence of surface waves on the flow field. The results show that the presence of surface waves significantly affects the distribution of turbulent kinetic energy near the free surface. The third case in which both the free surface waves and part of the ship hull were included is the first of its kind. Although only a small part (30\%) of the hull was included, this simulation has important benefits for the calculation of bubble trajectories in the very near wake of the ship. This is because most of the bubbles in the wake are generated on the surface of the ship hull and restrained in the boundary layer while they are being transplanted into the wake . For this case, calculated velocities at a cross section were compared with measurements. There is a qualitative agreement between the two although a further analysis would be needed to clarify certain issues.

In summary, with all the techniques presented in the present work, performing large eddy simulation of high Reynolds number ship wake flows seems possible and promising.

\subsection{Recommendations}

Accuracy and efficiency are the primary issues in large eddy simulation of high Reynolds number flows. The program used in this study is second order accurate. To achieve a better accuracy, higher order discretization schemes should be investigated. 
To perform LES of high Reynolds number flows, sufficient grid resolution has to be used to capture most of the energy-containing eddies. A single processor run will hardly provide enough capability to satisfy this demand. Thus, parallel computation with domain decomposition technique are desirable. Parallelizing the current LES program has been attempted and some preliminary results are included in Appendix A.

Free surface with waves is one of the most significant features in ship wake flows. An account of its shape and dynamics using an adequate physical model is more appropriate than using symmetry-boundary conditions with ship. Wave breaking is another important fact in the surface ship wake, which has significant effects on the turbulent flow field. However to include this phenomenon in the LES is rather difficult and should be undertaken as a long term future work.

Density stratified flows are common in the ocean, especially in the estuaries. When density stratification is present it may create a complicated flow pattern with vertical gradients in temperature and salinity. The interaction between temperature and salinity gradients has a notable effect on the velocity field and turbulent quantities (Jin and Zhang, 1992), which in turn will affect bubble dynamics. Therefore, it would be of a definite advantage to include the salinity and thermal stratification effects in the future LES of ship wakes.

Finally for surface ship wake simulations, the effects of free-surface/turbulence interaction, waves, and stratification should be eventually included. Fortunately, when using the one equation model SGS model, it is possible to account for some of these effects through the explicit modeling of dissipation rate of the subgrid turbulent kinetic energy. 


\section{Appendix A}

\section{Parallel Computation}

In this study the parallel implementation of large eddy simulations (LES) was performed by using MPI standard. In Section A.1 methodology for a domain decomposition technique on distributed memory computer platforms was proposed. In this methodology the decomposition can be realized on different levels of complexity, depending on the flow characteristics and efficiency considerations. In Section A.2, the results of realization of both parabolic (one-way) and elliptic (two-way) decomposition on a cluster of workstations at WVU were presented.

\section{A.1 Domain Decomposition Strategy}

The conventional domain decomposition technique for elliptic problems is realized through a two-way exchange of data at the boundaries of the domains (Simon, H.D., 1992; Dihn, Q.V., Glowinski, R. and Periaux, J., 1984) as illustrated in Fig. A.1(a) for a one-dimensional problem. This guarantees the physical integrity of the solution and the convergence to the corresponding single domain case. However, this strategy may carry an excessive communication overhead for three dimensional CFD simulations. If the problem is parabolic in one of the spatial directions one can employ a more efficient parabolic communication approach illustrated in Fig.A.1(b). This may reduce communication overhead considerably when non-blocking send-routines are used. 


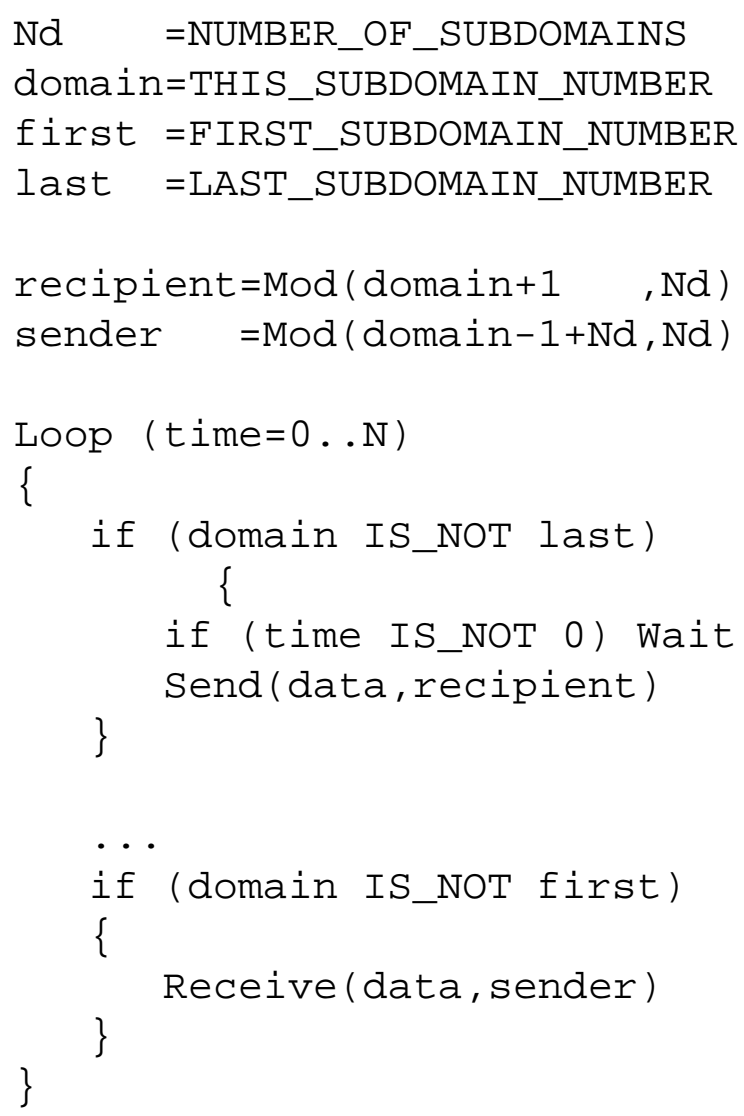

Table A.1: Parabolic domain decomposition algorithm

Considering these factors, the first parallel version of the LES code has been implemented using parabolic data exchange. This approach is valid for ship-wake applications since the flow in most of the ship-wake region has a parabolic character. Even though small recirculation zones exist in the proximity of ship's stern they do not stretch far enough and can be contained entirely within the first sub-domain.

To exploit the parabolic nature of the solution the computational domain of the whole wake should be subdivided into $N d$ sub-domains by cutting it with planes normal to the ship-velocity ${ }^{1}$. Figure A.2 illustrates it on the example of two sub-domains. Data exchange between the domains occur at the plane $E H$ in the figure.

Table A.1 provides an outline of the message-passing algorithm that should be used

\footnotetext{
${ }^{1}$ Ship velocity is presumed constant
} 
to accomplish the parabolic data exchange. The Send and Receive statements are introduced into the main time-loop of the flow-solver routine, where time is an integer time-step counter. Recipient and sender domain numbers are computed using the division by modulus function $\operatorname{Mod}(a, b)$. Ellipses ("...") inside the Loop in Tab. A.1 designate all the rest of the flow-solver routines not related to message-passing functions. The $W$ ait function is introduced to enable the usage of non-blocking Send's, which avoids unnecessary waiting for data-receipt confirmations and allows the processor to perform other flow-solver procedures while the data are being sent. This implementation of the domain-decomposition technique is rather simple and straightforward. It also carries a minimum communication overhead and is suitable for ship-wake applications.

However, the parabolic domain decomposition algorithm is only the first stage of our MPI implementation. The drawback of the parabolic exchange scheme is the necessity to provide additional outlet boundary conditions for each domain, which can alter the character of the flow close to the domain outlet, as compared to the elliptic exchange scheme. To avoid the influence of this distortion on the flow-field the communication plane should be set at some distance from the outlet plane. Thus some of the memory space and the processing time is inevitably lost. Moreover, only by using elliptic message transfer can one apply domain decomposition technique in non-parabolic directions which is necessary for geometrically complex flows. For these reasons the elliptic communication mechanism is currently under development. The message-passing algorithm of elliptic data exchange is similar to the parabolic one expect that the messages are transferred in both way between the two domains. The outline of the elliptic message-passing is demonstrated in table A.2

\section{A.2 Implementation on the Beowulf cluster}

The parallelizing methodology described above was implemented on a Beowulf cluster of DEC-Alpha workstations at the CFD laboratory of WVU (Fig.A.5). 


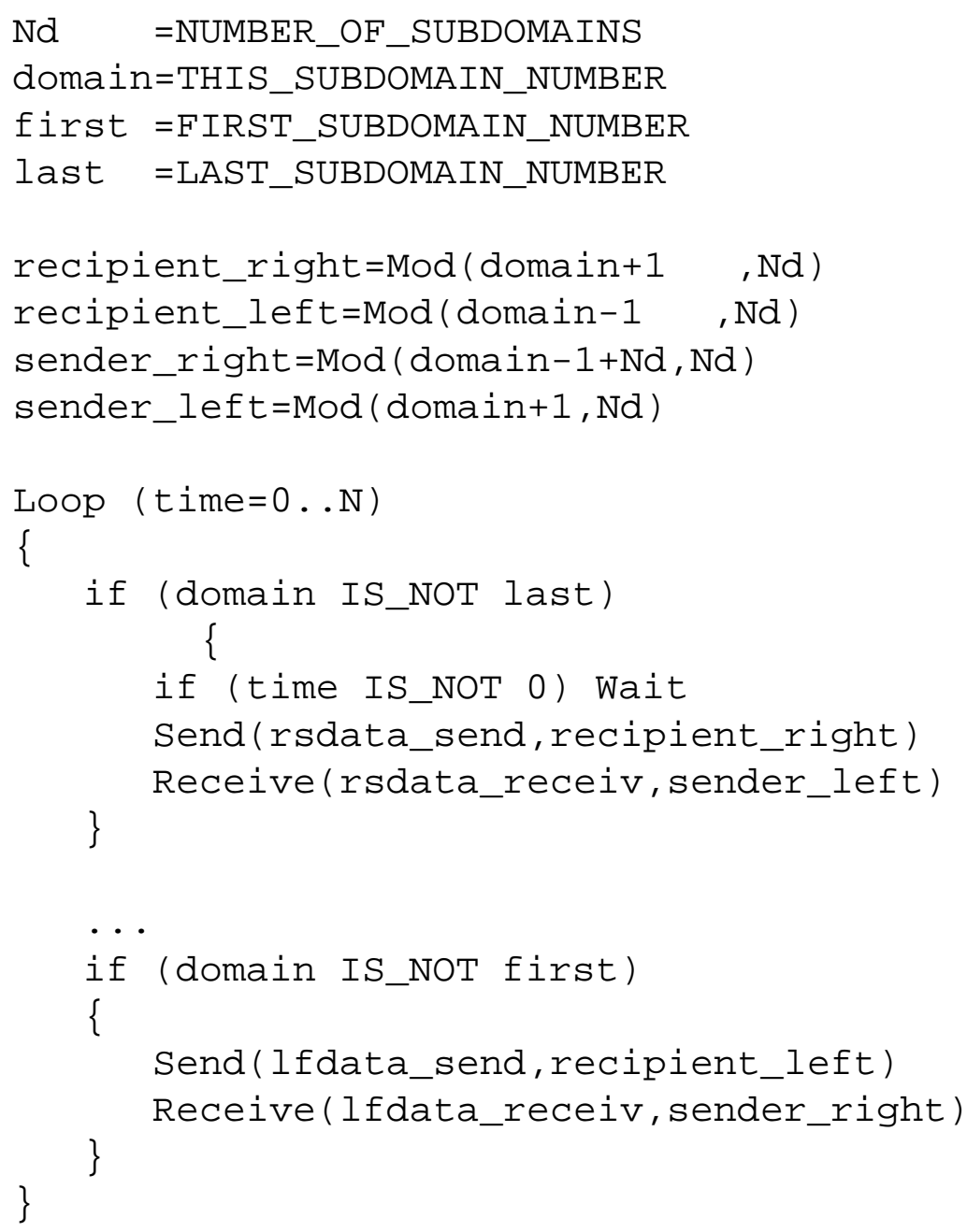

Table A.2: Elliptic domain decomposition algorithm 
The cluster is setup to run the Linux operating system with the MPI interface freely available from www.mcs.anl.gov/mpi/mpich/download.html.

\section{A.3 Test Simulation}

A simulation was successfully run on 2 processors of the cluster with the message-passing schemes described above. The sketches of both parabolic and elliptic data transferring are shown in Fig.A.3.

A flat plate wake was used to test the parallelizing computation approach. The geometry and numerical scheme can be found in Chapter 4. the first domain starts from the same plane as in Chapter 4. Inflow boundary is also created by using RFG technique.

As shown in Fig.A.3(a), for parabolic decomposition both domains have their own outflow boundary. So, to avoid the influence of outflow boundary condition, the second domain starts far away from the outflow boundary, about half of the domain in this test case.

The first domain stretches from 0 to $0.4 m$, while the second - from $0.2 m$ to $0.8 m$. The other two dimensions are of the same size for both domains.

For elliptic decomposition as shown in Fig. A.3(b), the information exchange is happening between the last two nodes of the first domain and the first two nodes of the second domain, i.e., the last node of the domain 1 gets messages from the second node of domain 2, and the first node of domain 2 gets messages from the node next to the last one of domain 1. In this case both domains can be of the same size, i.e., $0.4 \mathrm{~m}$.

The grid size for both domains is $18 \times 18 \times 18$ with slight stretching in both the streamwise and vertical directions. The other boundary conditions applied here for both domains are the same as those described in Chapter 4.

Next, the streamwise velocity contours of both parabolic and elliptic schemes are shown in Fig.A.4. There is a slight difference between each other because in parabolic scheme the outflow boundary has to be applied for both domains. This in turn will effect the flow inside 
the domain.

\section{A.4 Scalability Analysis}

The scalability analysis has been performed by implementing the MPI technique on different domain decompositions (Osman et al., 2000). It indicted that the speedup is almost linear proportional to the processors (domains) being used.

\section{A.5 Summary}

The following conclusions can be drawn from the results of this chapter:

1. Customized message-passing routines were written and tested on the basis of MPI to provide a simple language-independent interprocessor communication.

2. Both parabolic domain decomposition and elliptic domain decomposition methodology can be used for efficient parallelization of CFD codes for wake flow applications.

3. Scalability analysis indicted that the speedup is almost linear proportional to the processors (domains) being used. 


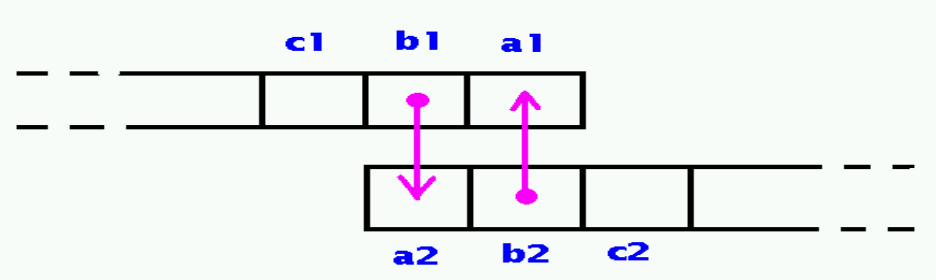

(a) Elliptic decomposition

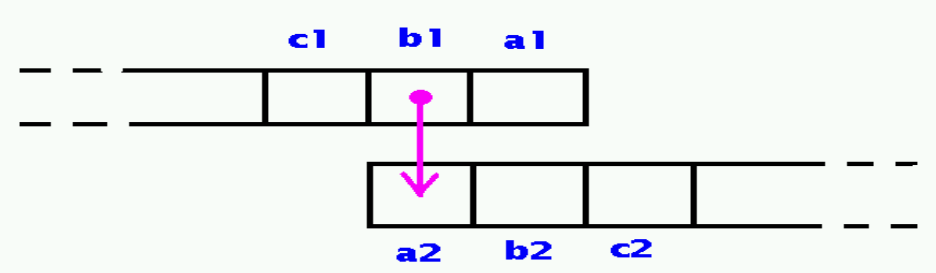

(b) Parabolic decomposition

Figure A.1: Domain decomposition strategy $a, b, c$ - cells of the grid in different computational domains 


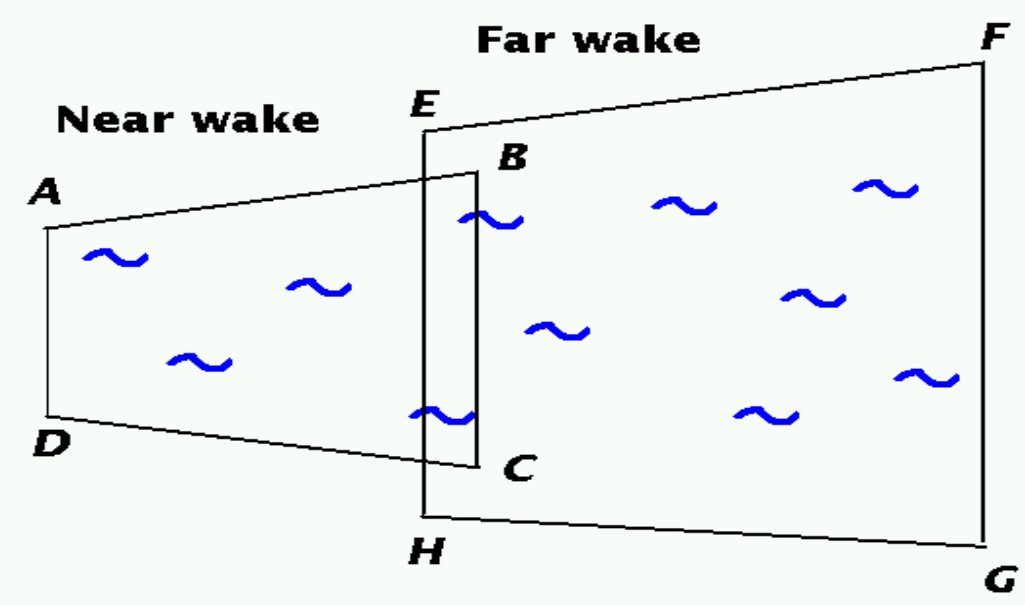

Figure A.2: Wake decomposition 


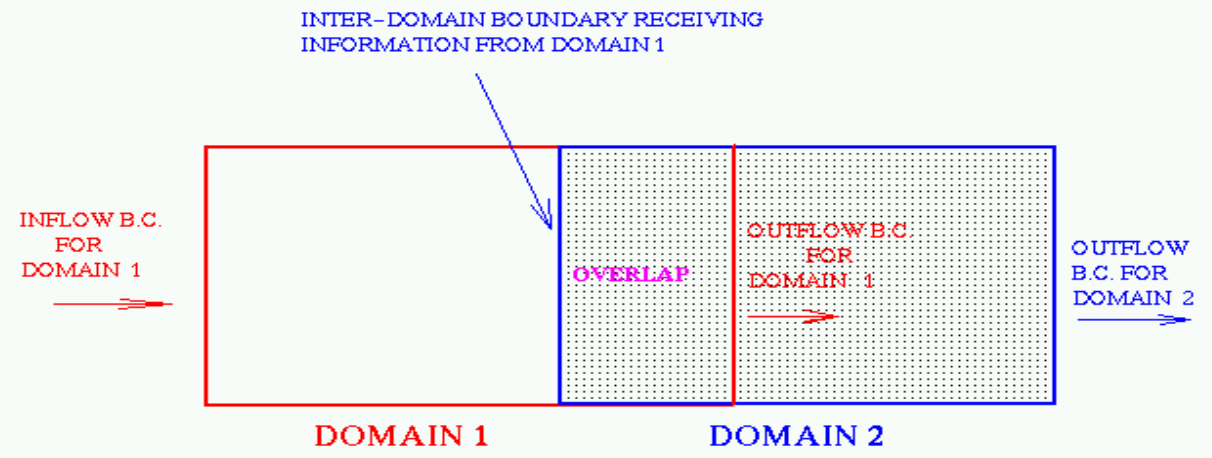

(a) The demonstration of parabolic decomposition scheme

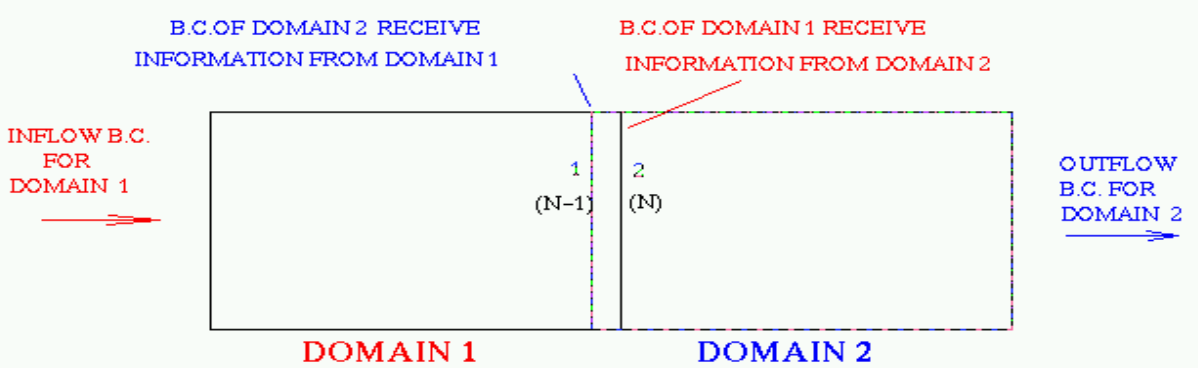

(b) The demonstration of elliptic decomposition scheme, 1,2 - the first and second grid of Domain 2; N-1, N - the last two grid of Domain 1

Figure A.3: The demonstration of domain decomposition scheme 


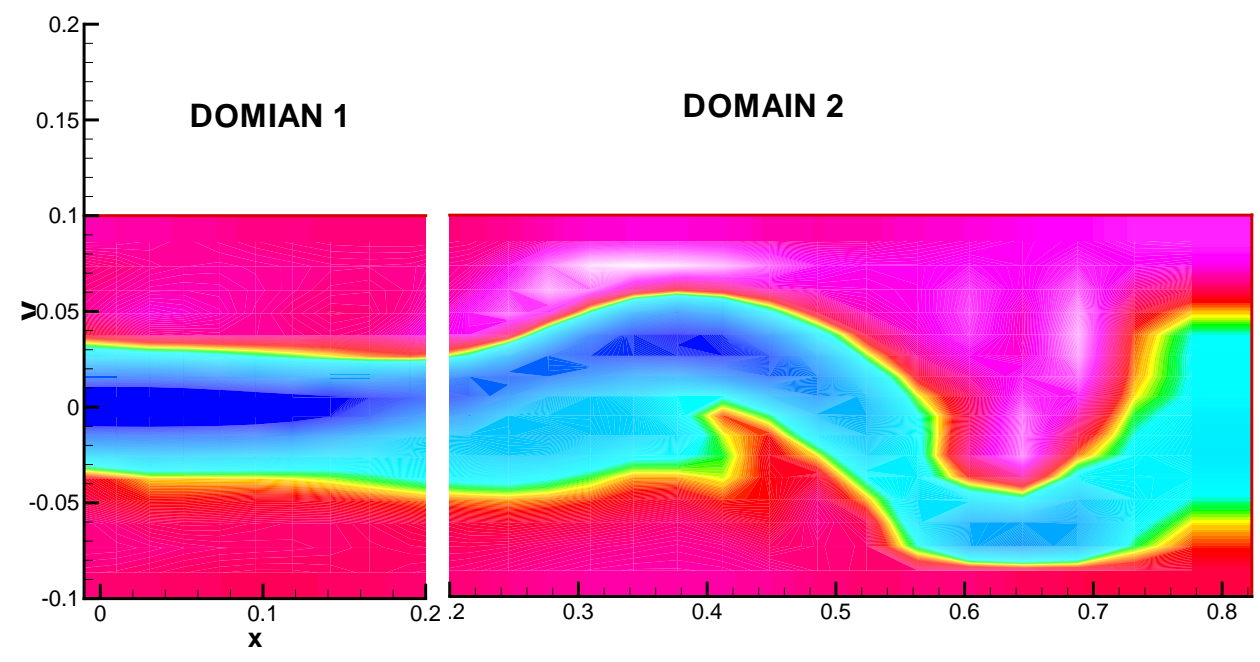

(a) The streamwise velocity contours of parabolic decomposition scheme

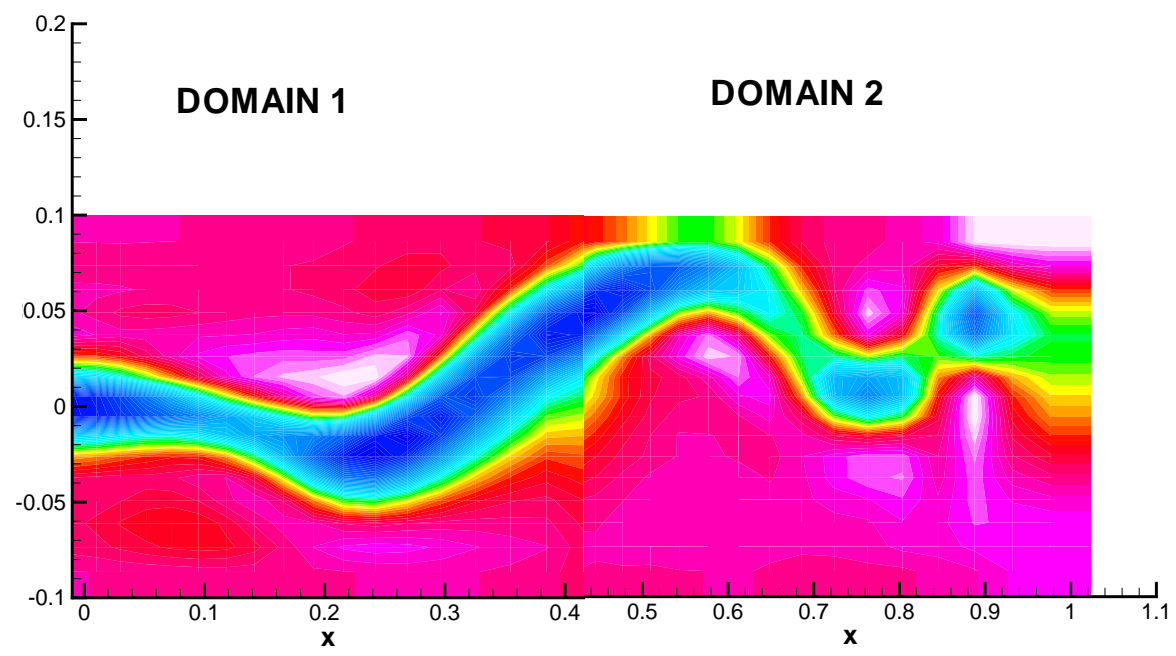

(b) The streamwise velocity contours of elliptic decomposition scheme

Figure A.4: The streamwise velocity contours 


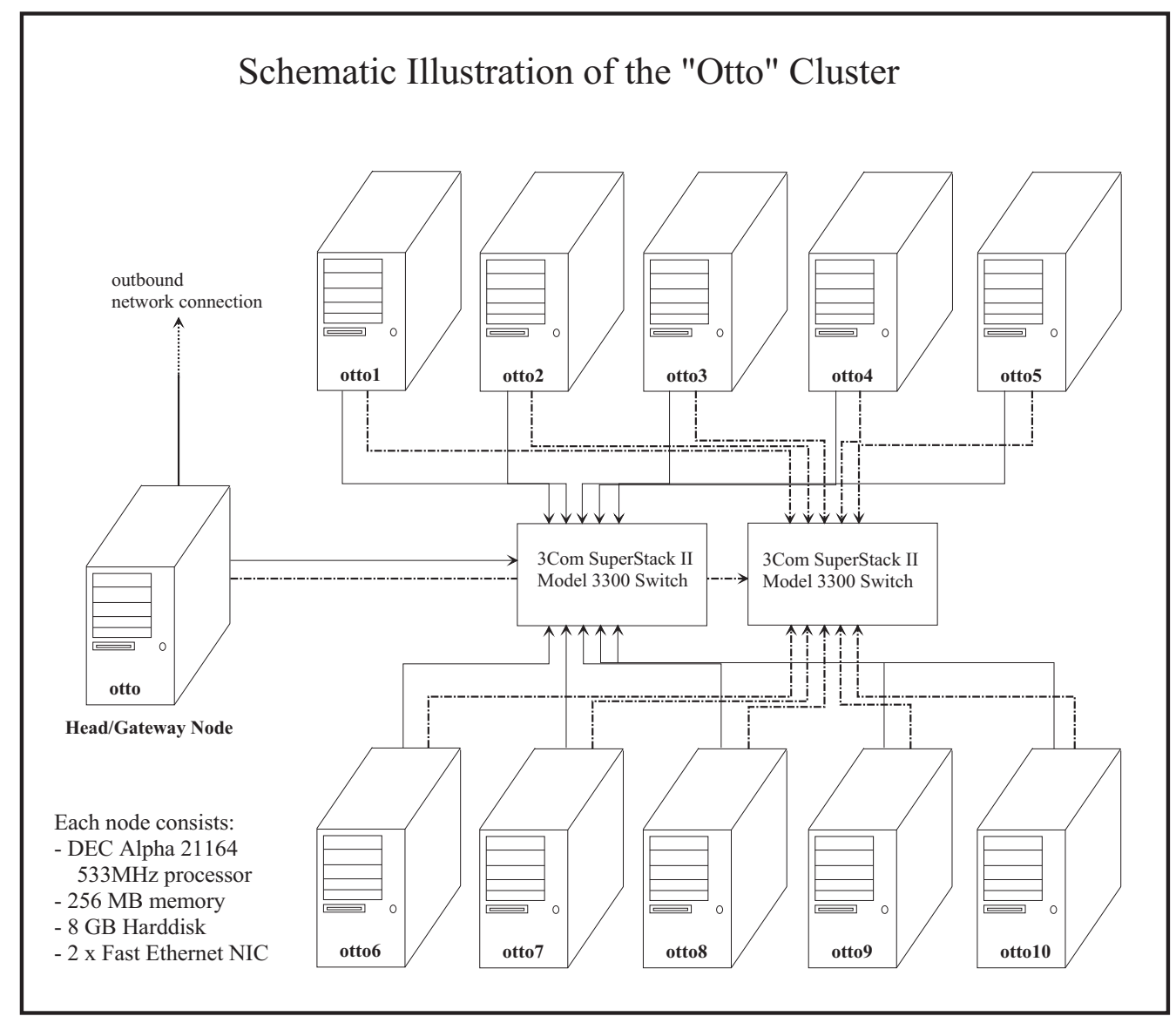

Figure A.5: A cluster of DEC-Alpha workstations at the CFD lab of WVU 


\section{Appendix B}

\section{Introduction of the LES Code}

The LES code was originally developed by Zang et al. (1994) at Stanford University. As has been verified in this study, it is a robust code with satisfied accuracy and efficiency. However, with no instruction and very few comments, it is very difficult to use. Therefore it is worthwhile to summarize the code and create a user manual.

\section{B.1 Flow Chart}




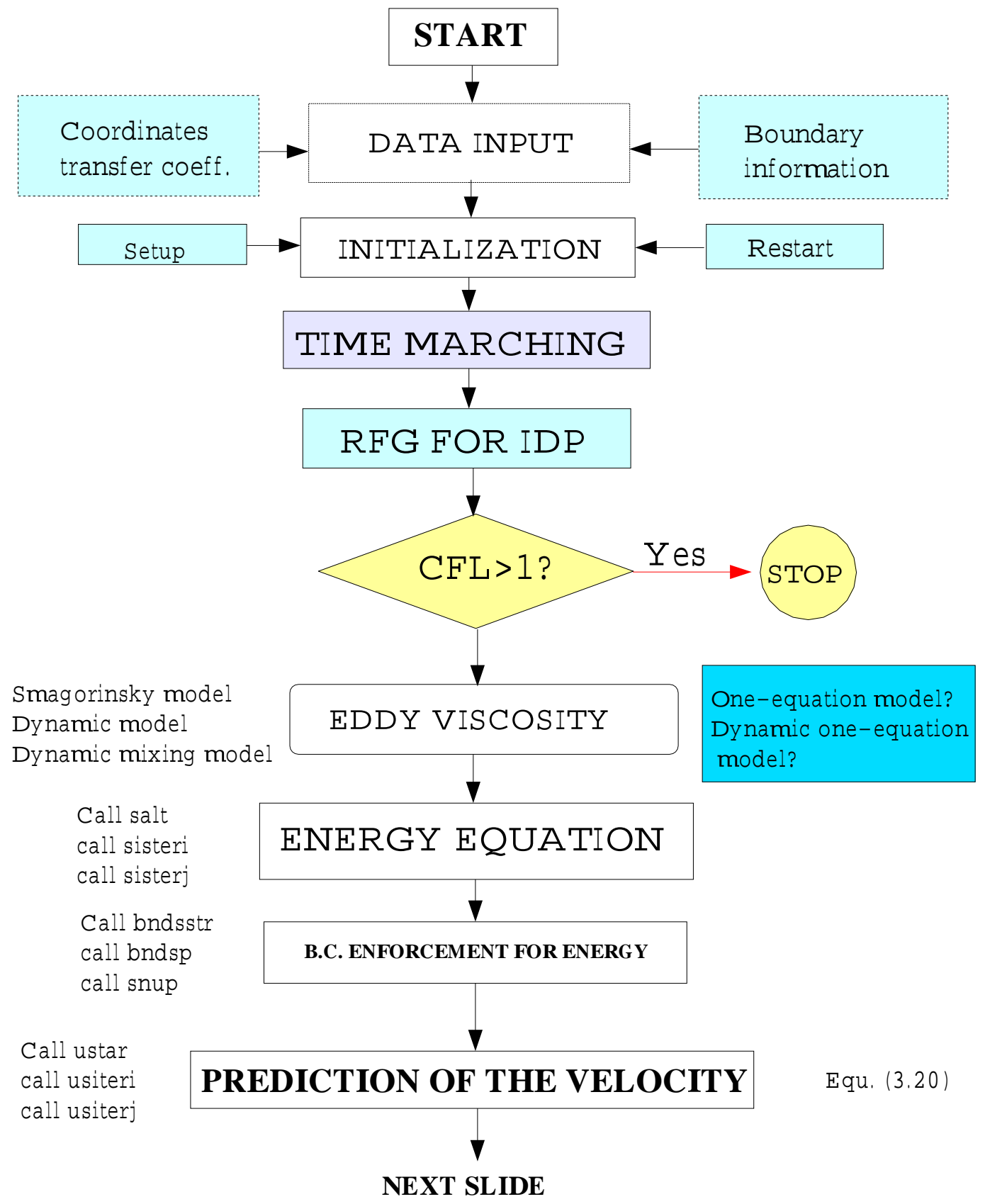

Figure B.1: The flow chart of the LES code - A 
Call source

Call ucorr

Call bndup

Call presr

Call bopg

call relax

call resid

call check

call rsrtct...

Call ucorr

Call output

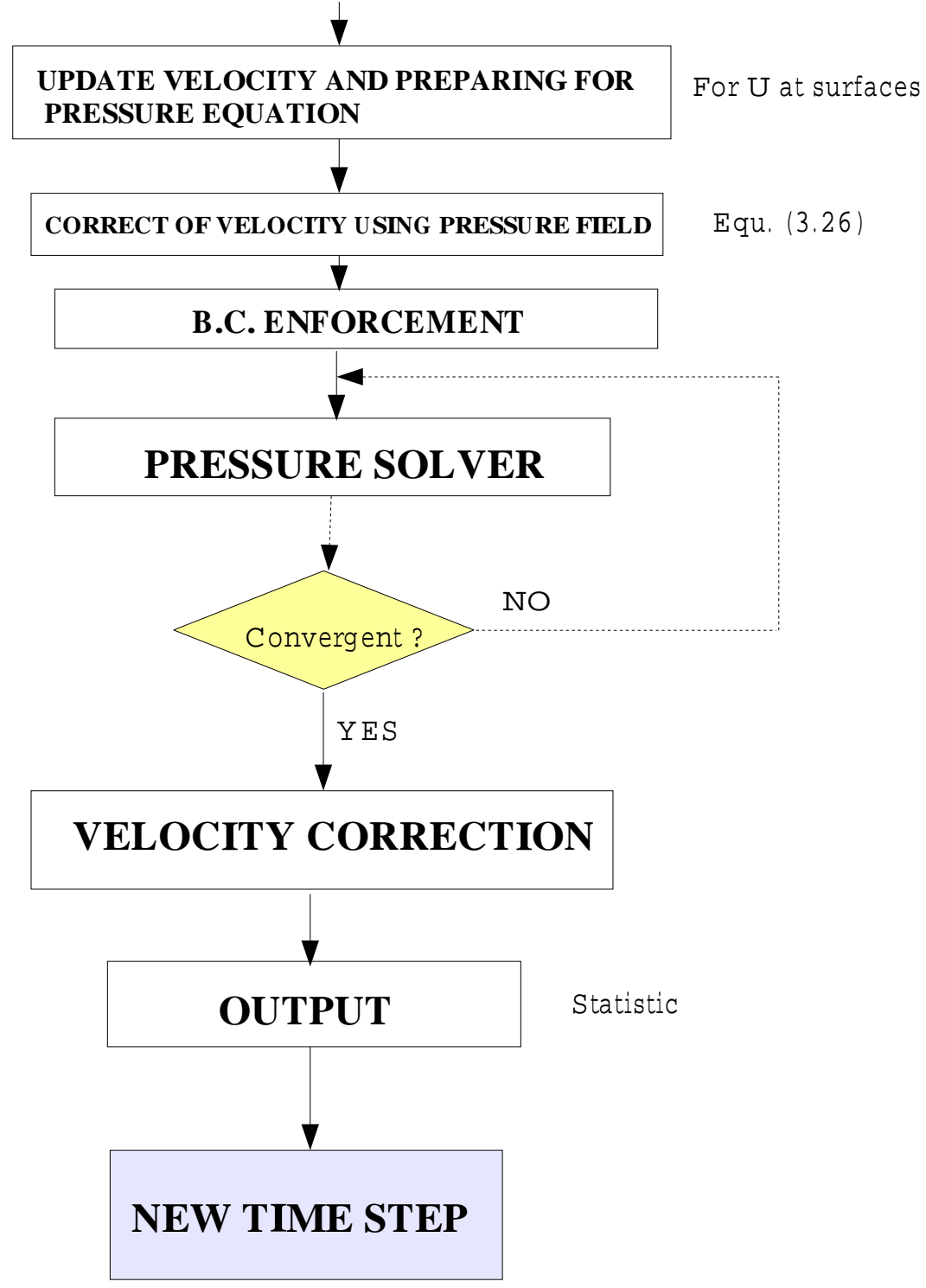

Figure B.2: The flow chart of the LES code - B 


\section{B.2 Description of the Subroutines}

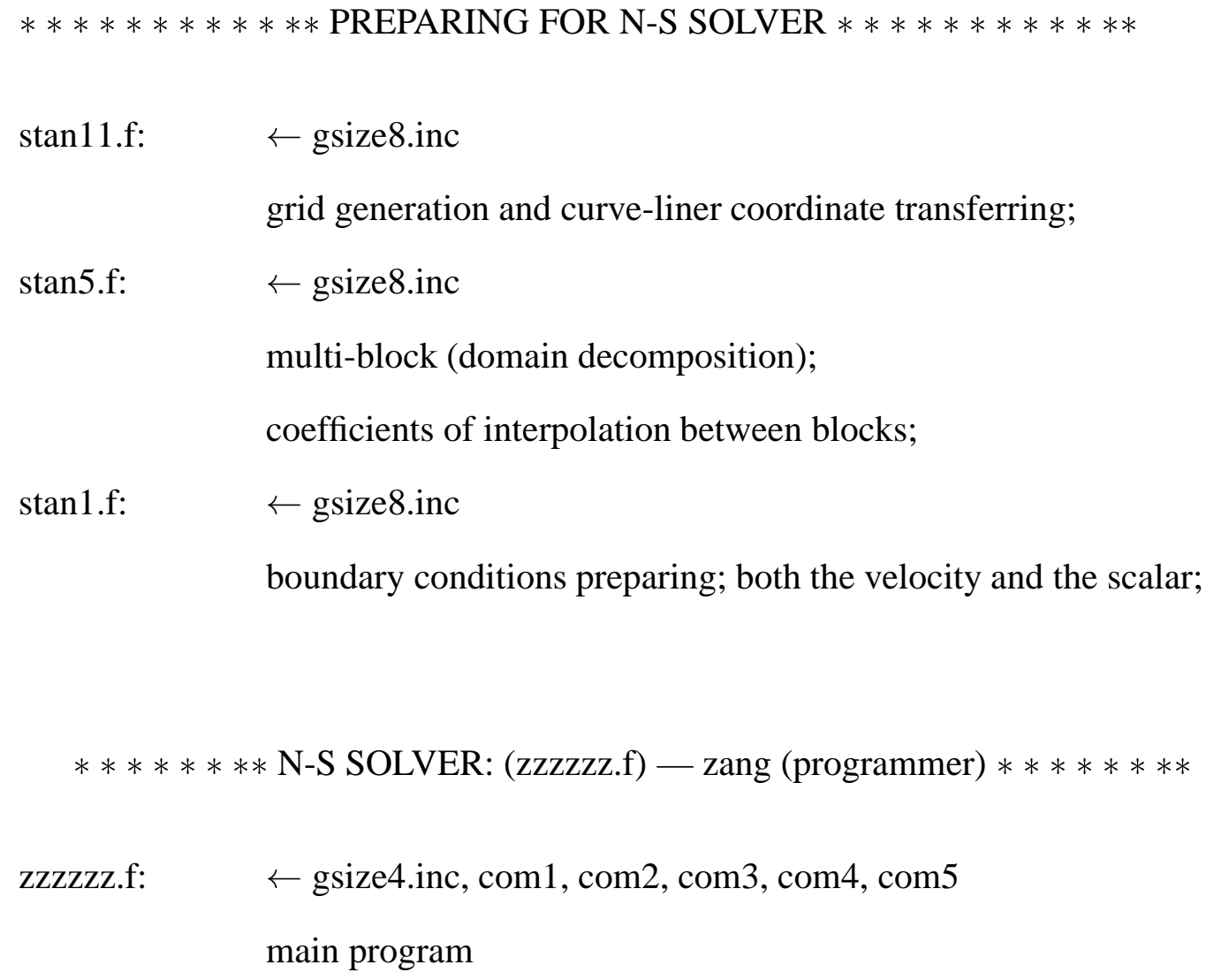

openc.f:

open the data files, both input and output (history);

setloc.f:

make equivalent one-dimension storage for global variables;

metricin.f:

read in data;

bcmet, matrix period $\mathrm{BC}$ enforced;

initial.f:

initialize the flow, or restart it;

cofin $\Rightarrow$ cofmg, compute the ap(19) for muti-grid;

tick.f: 
control routine;

time marching;

solver.f:

N-S solver;

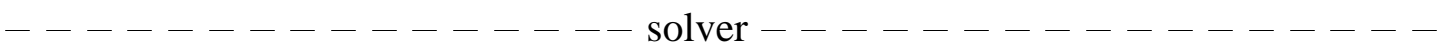

eddy.f:

calculate the strain rate tensor;

calculate the turbulent eddy viscosity $\left(\nu_{T}\right)$ for momentum equation;

calculate the eddy diffusivity $\left(\kappa_{T}\right)$ for the scalar equation;

$\mathrm{s} 23 \Rightarrow \nu_{T} ; \mathrm{sab} \Rightarrow \kappa_{T} ;$

Smagorinsky model; dynamic model; mixed dynamic model;

two - parameter mixed dynamic model; Salvetti, et al. (1997)

ssmm.f:

calculate the similarity term if mixed model is applied;

the last term in Eq. (3.5); and Eq. (3.7);

$s s t(i, j, k, m), m=1,3$ for momentum equation;

$\operatorname{sst}(i, j, k, 4)$ for scalar equation;

tensor.f:

calculate the turbulent eddy viscosity $\left(\nu_{T}\right)$ through one-equation SGS model;

backscatter is accounted by applied RFG; fluctuation.f;

source term (include production and dissipation) for k-equation is

calculated and stored in $s s t(i, j, k, 4)$;

salt.f: 
calculate the convection term in scalar equation by using SHARP; cross diffusion term form Eq. (3.16); (explicit) similarity-part is added in; in one-equation SGS model: source term; Crank-Nicolson for the diagonal duffusive term; Eq.(3.15); marching on $k$ direction; Eq.(3.27) $\leftarrow$ approximate factorization;

siteri.f:

scalar equation, marching on $i$ direction; Eq.(3.27) $\leftarrow$ approximate factorization;

siterj.f:

scalar equation, marching on $j$ direction; Eq.(3.27) $\leftarrow$ approximate factorization;

bndsstr.f:

boundary condition for scalar-changing $\Delta s$;

bnss.f:

Inter-BC; MPI reference;

snbc, interpolate between blocks;

snup.f:

snew $=s+d s$

bndsp.f:

physical boundary for scalar is enforced;

bndsn.f:

Inter-BC ;

get data form the next block and saved as $s s t(i, j, 4, i g)$, which is used in siteri.f;

ustar.f:

calculate the convection term in scalar equation by using QUICK;(3.10) 
cross diffusion term form Eq. (3.13); (explicit)

Adams-Bashforth applied;

similarity-part is added in; from ssmm.f;

Crank-Nicolson for the diagonal duffusive term; Eq.(3.15);

marching on $k$ direction; Eq.(3.25) $\leftarrow$ approximate factorization;

coefficients modified at the boundaries;

usiteri.f:

momentum equation, marching on $i$ direction; Eq.(3.25) $\leftarrow$ approximate factorization;

usiterj.f:

momentum equation, marching on $j$ direction; Eq.(3.25) $\leftarrow$ approximate factorization;

bndustr1.f:

bndustr2.f:

boundary condition for velocity changing $\Delta u_{i}$;

bnsu.f:

Inter-BC; MPI reference;

uwbc2, interpolate between blocks;

get $d u_{i}$ from other blocks;

bnduw2.f:

Inter-BC; MPI reference;

get $u^{*}-u(n)$ from other blocks and store in $u b y(i, j, m v, m b)$

source.f:

update the velocity $u, v, w$ after Eq.(3.25);

interpolate (fourth order) the velocity on the surfaces;

calculate the contravariant velocity using the interpolate velocity; 
(intermediate, $U_{m}^{*}$ in Eq.(3.30));

calculate the source term for Eq.(3.31); store in $\operatorname{loc}(33)$

calculate $\left(U-U^{*}\right) / d t$ at the boundaries; RHS of Eq.(3.41) utosst.f:

save the velocities temporary at sst which was used in eddy.f;

ucorr.f:

update the velocities after Eq.(3.26);

$\mathrm{dp} / \mathrm{dx}, \mathrm{dp} / \mathrm{dy}, \ldots$, are $\frac{\partial \phi}{\partial \xi} ;$

at boundary 2 nd order interpolation is applied;

faces $\rightarrow$ edges $\rightarrow$ corners $\rightarrow$ interior;

update the contravariant velocities after Eq.(3.30); this step active

only after the pressure iteration has been finished;

bndup.f:

enforce the physical boundaries;

compatible with those in ucorr.f

bnduwn.f:

Inter-BC for velocities; MPI reference here;

calculate pressure source terms for inter-BC;

uwbc1 get velocity and store it at $u s b(i, j, m v, m b)$;

bvelsr 2 compute contravariant velocity using $u s b$ for Inter-BC;

bpnum.f:

Inter-BC for pressure; MPI reference here;

$\mathrm{pnbc} \Rightarrow \mathrm{pp}, \mathrm{pm}$, it seems no difference between pp and pm; MPI.

$\mathrm{ppbc} \Rightarrow$ update pressure $\mathrm{pp}$ and $\mathrm{pm}$ for Inter-BC,

presr.f:

pressure solver;

in bcpg.f, $\frac{\partial p}{\partial \xi}, \ldots$, at the 
boundary is calculated; for physical BC, see, Eq.(3.41);

for inter-BC, $\mathrm{CD}$ is used;

it seems pp, pm from bpnum are not used; if this is

the case, MPI for bpnum is not necessary;

call relax for smoothing;

call resid for residual;

for multi-grid, do V-cycle;

enforce $\mathrm{BC}$ on the finest level;

smoothing on the finest level;

inner loop for MG:

get residual on the finest level;

call rstrct to restrict the residual on one coarser level;

smooth the residual on current level (ndown loops);

(the coef. from cofin $\leftarrow$ cofmg);

till the coarest level; finish down of V-cycle

use interp to interpolate $\mathrm{p}$ to get it at a finer level;

smooth p at current level (nup loops);

till the finest level; finish up of V-cycle;

enforce $\mathrm{BC}$ for $\mathrm{p}$;

finish inner loop.

relax.f:

bndp.f: update the $\mathrm{p}$ at all the ghost nodes; only on the surfaces;

the corners and edges are updated in interp;

using $\frac{\partial p}{\partial \xi}, \ldots$, 
from bcpg.f to get p; symmetry BC and periodic BC are enforced;

point Gauss-Seidel smoothing when intp=1;

ZEBRA/4-color smoothing when intp=2; (as my note);

notice the boundary points;

$a p(19)$ is the 19 coefficients for pressure equation,

see(3.137) Perng (1990);

resid.f:

get the residual for pressure equation;

residual of pressure gradient at the boundary;

rstrct.f:

restrict correction from fine grid to one coarser level;

3 -D, $8 \Rightarrow 1$, points are repeat twice; thus divided by 2 ;

2-D, 4 $\Rightarrow 1$, no point repeat; Fig.4.3, Perng (1990);

pressure gradient at the boundaries;

corners not included;

call relax after this; smoothing correction on current level;

interp.f:

interpolate pressure from coarse grid to finer grid;

corners and edges are updated here;

liner-interpolation;

bndun.f:

Inter-BC update for the new time-step;

unbc - for MPI;

ckdiff.f:

calculate pressure difference and mass residuals; 
df $m$ is the maximum of pressure changing $=\sqrt{p_{\text {max }}^{2}}$;

$d f v$ is the average $=\sqrt{\sum p^{2}}$;

rpm is the maximum of mass residuals;

$r p v$ is the average of mass residuals;

output.f:

output file;

getcfl.f:

calculate $C F L$; 


\section{B.3 Using the Code}

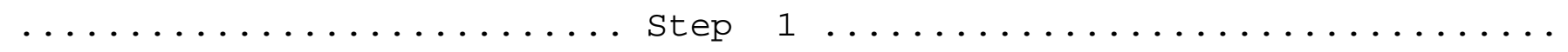

Step1: Compile and run "stan11.f" --- Grid generation and coordinates transferring

do not change anything at the part before "subroutine coord"; in the part of subroutine coord (grid generation), anything can

be changed, the only thing needed to get back is "x2" which is the grid coordinates;

"gsize8.inc" is an include file for "stan11.for";

il: nodes at $x$ direction;

jl: nodes at y direction;

$\mathrm{kl}$ : nodes at $\mathrm{z}$ direction;

$i j m: \max (i l, j l, k l)$;

ng: block number; (always be 1 in our case)

"cmin2" is the input file for "stan11.for";

8 : switch, always be this number.

0.45 : useless

$1 \quad:$ ng, block number

825050 : grid size

132355 : nb, boundaries, description bellow

$0 .-0.1-0.3$ : starting points for $x, y$ and $z$;

1.00 .10 .3 : length of domain, Lx, Ly/2, Lz/2

$001 \quad: 0$-- nonuniform, 1 -- uniform

0.0010 .020 .02 : parameters for grids distribution

0.00030 .040 .04 : the size of first node, $d x$, dy dz;

$0.0010 .001 \quad$ : useless

nb = 1, Dirichelet brdy (user specifies velocity);

2, Neumann brdy (gradient-free);

3, symmetric boundary;

4, axisymmetric in theta-direction

(specific geometry)

5, periodic brdy;

6 , inter-grid brdy; 
notice, in "gsize8.inc" and "cmin2", grid number must match.

"xx" for checking the grid quality. first part: $x, d x$ second part: $y, d y$ third part: $z, d z$

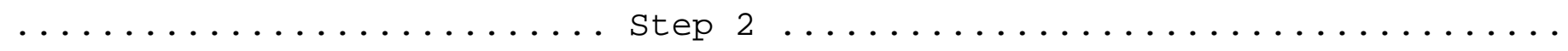

Step2: Compile and run "stan5.f"---computer the coefficients for interpolation at the interfaces between blocks.

to creat "coef.in" for $\mathrm{N}-\mathrm{S}$ solver;

"gsize8.inc" is an include file for "stan5.for";

"cfin" is the input file for "stan5.for";

1 : nb at surface 1 --- left (inlet)

2 : nb at surface 2 --- top

2 : nb at surface 3 --- right (outlet)

2 : nb at surface 4 --- bottom

5 : nb at surface 5 --- behind

5 : nb at surface 6 --- front

$3: 3-D$

Step3: Compile and run "stan1.f" --- compute the initial and boundary conditions.

the content of this code needs to be changed for different flow; the purpose is to specify the velocity or the scalar variable on all six surfaces, or turbulence intensities if RFG is used to provide unsteady inflow boundary;

"gsize8.inc" is an include file for "stan5.for";

"ubin" is the input file for "stanl.for";

it does not need to be changed it now, because all the 
information needed is either specified or read in;

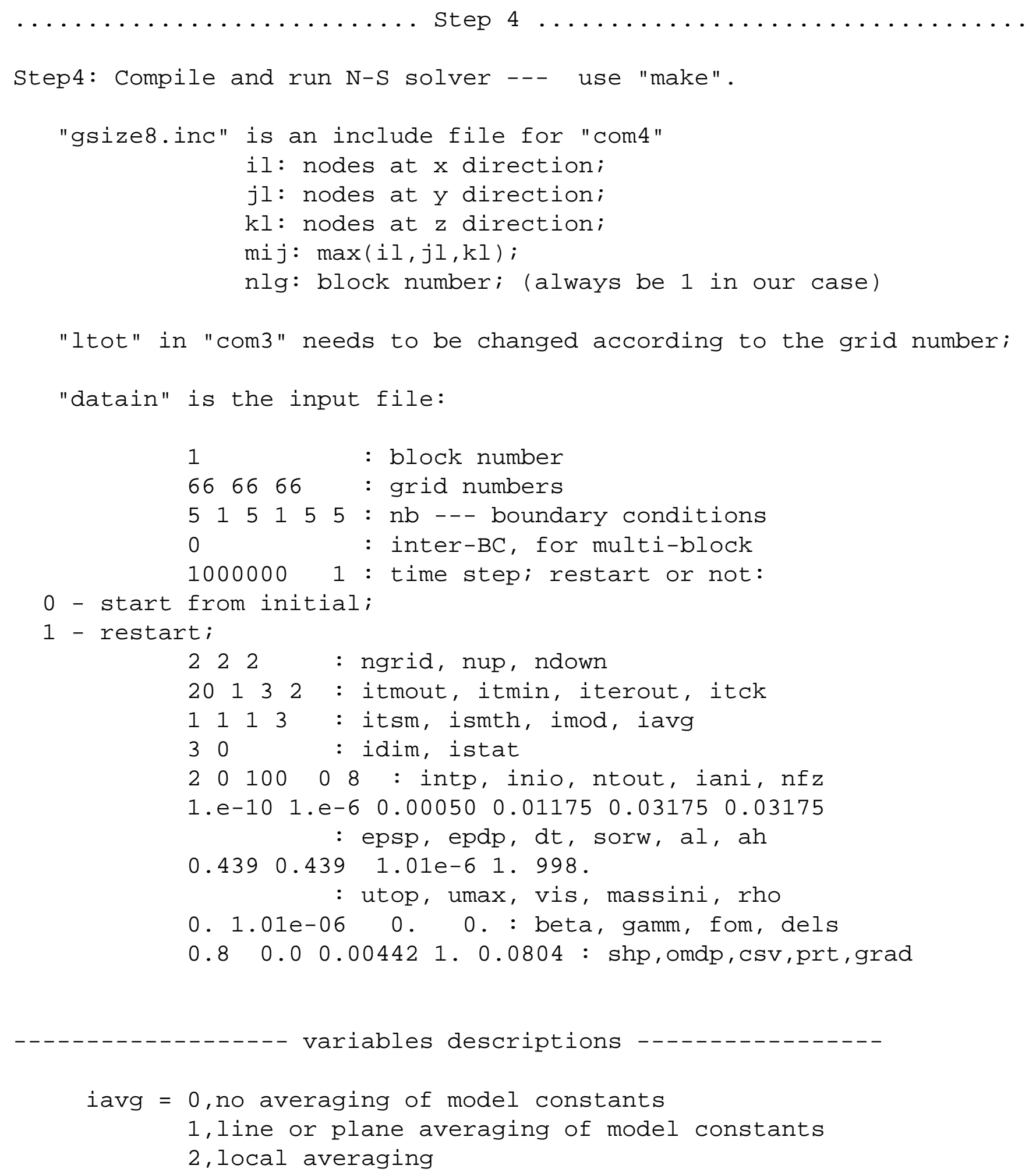




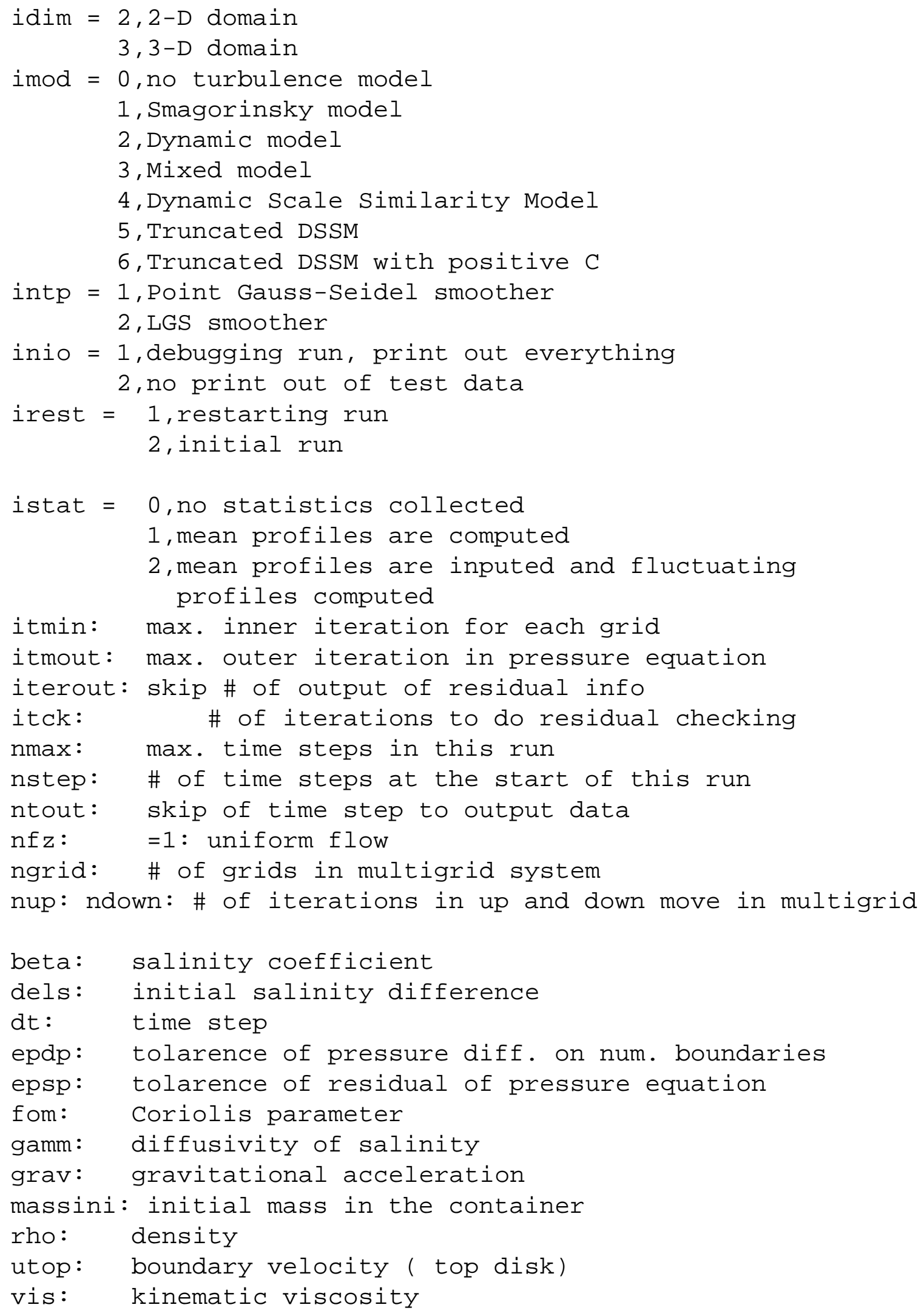


grad: pressure gradient

OUTPUT :

"usshi": velocity

"vort": vorticity

"up*" : velocity at one point 


\section{Appendix C}

\section{Calculation of the Energy Spectra}

The purpose of this Appendic is to write a simple program for the analysis of turbulence flow. The method is based on a Fourier transformation approach(Hayasa, 1999).

The energy spectrum of the U-velocity perturbation is calculated by the following formula

$$
\begin{array}{r}
S_{1}\left(\omega_{n}\right)=E\left[\frac{h_{T}}{M_{d}}\left|\sum_{m=1}^{M_{d}} U_{1}^{\prime}\left(t_{m}\right) \exp \left(-i \omega_{n} t_{m}\right)\right|^{2}\right]_{N_{d}} \\
\left(n=1, \ldots, M_{d}\right)
\end{array}
$$

Where $\omega_{n}=2 \pi n /\left(h_{T} M_{d}\right)$ is the nondimensional circular frequency, $M_{d}$ is the number of time series data, and $E[*]_{N_{d}}$ denotes the averaging over $N_{d}$ samples.

The energy spectrum can be converted into wave number domain as,

$$
E_{1}\left(\alpha_{n}\right)=U_{c} S_{1}\left(\omega_{n}\right)
$$

where $\alpha=\omega_{n} / U_{c}$ is the wave number.

The program is written in Fortran as follows: 


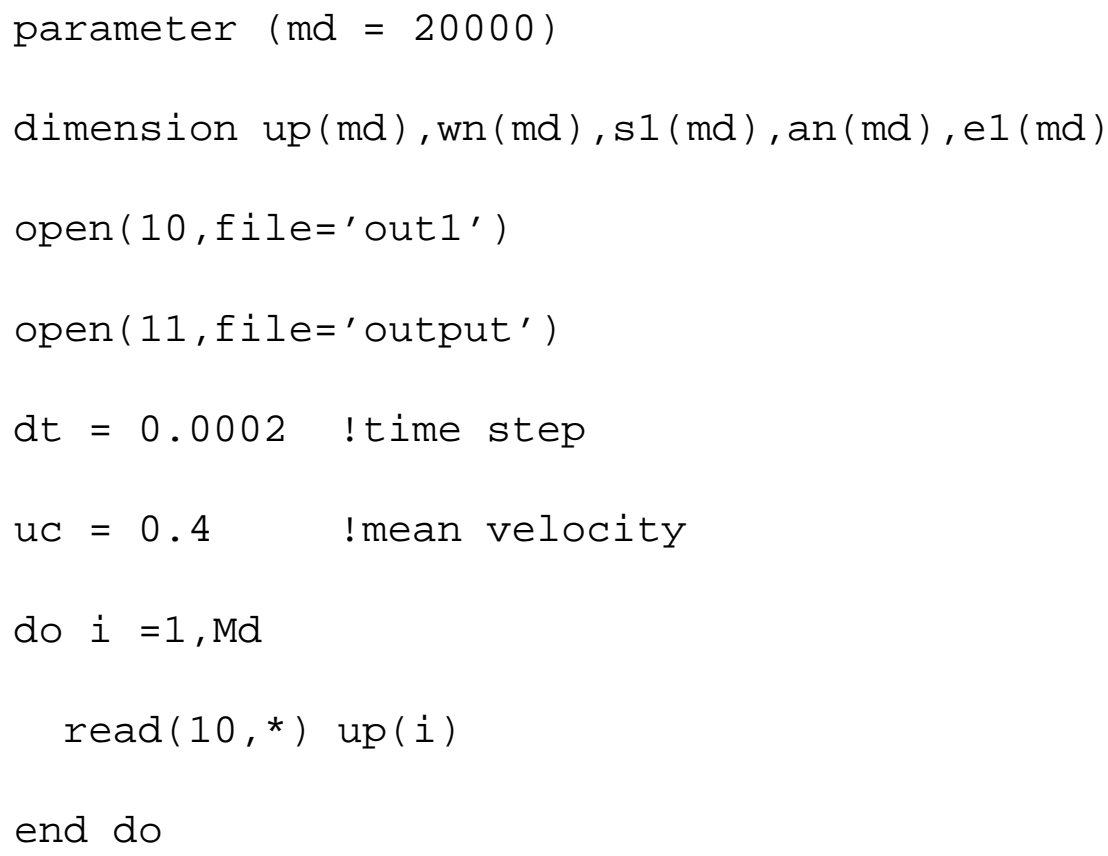




$$
\begin{aligned}
& \operatorname{an}(i)=\text { wn }(i) / u c \\
& \text { el(i) }=u c^{\star} \text { s } 1(i) \\
& \text { write }(11, *) \operatorname{an}(i), e 1(i) \\
& \text { end do }
\end{aligned}
$$$$
\text { end }
$$ 


\title{
NOMENCLATURE
}

\author{
$E_{1} \quad$ - energy spectrum of U-velovity perturbation in wave number \\ domain; \\ $E[*]_{N_{d}}$ - averaging over $N_{d}$ samples; \\ $h_{T} \quad$ - computational time step; \\ $h_{T} \quad$ - computational time step; \\ $N_{d} \quad$ - number of averaging data ; \\ $U_{c} \quad$ - mean velocity.
}

This algorithm has been tested by using two benchmarks. One is a sinusoidal function which is shown in Fig. C.1. The other one is the test data from the Kay (1988) database which has been widely used to verify the energy spectra algorithm. After the transformation, the resulted spectra for the first test case is shown in Fig. C.2. As expected, two peaks exist while it is zero everywhere else. For benchmark two, the results are presented in Fig.C.3. 


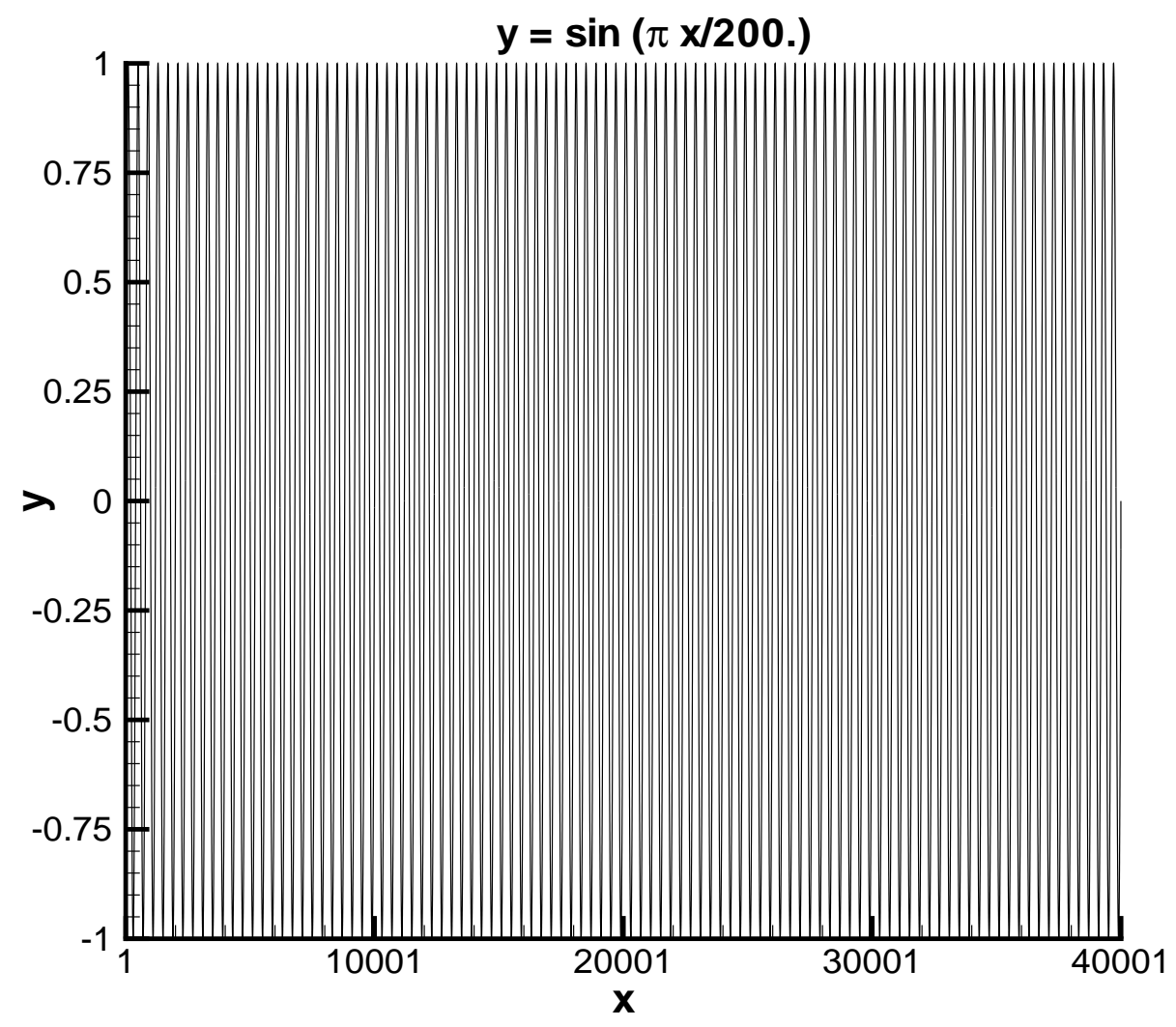

Figure C.1: The sinusoidal signals 


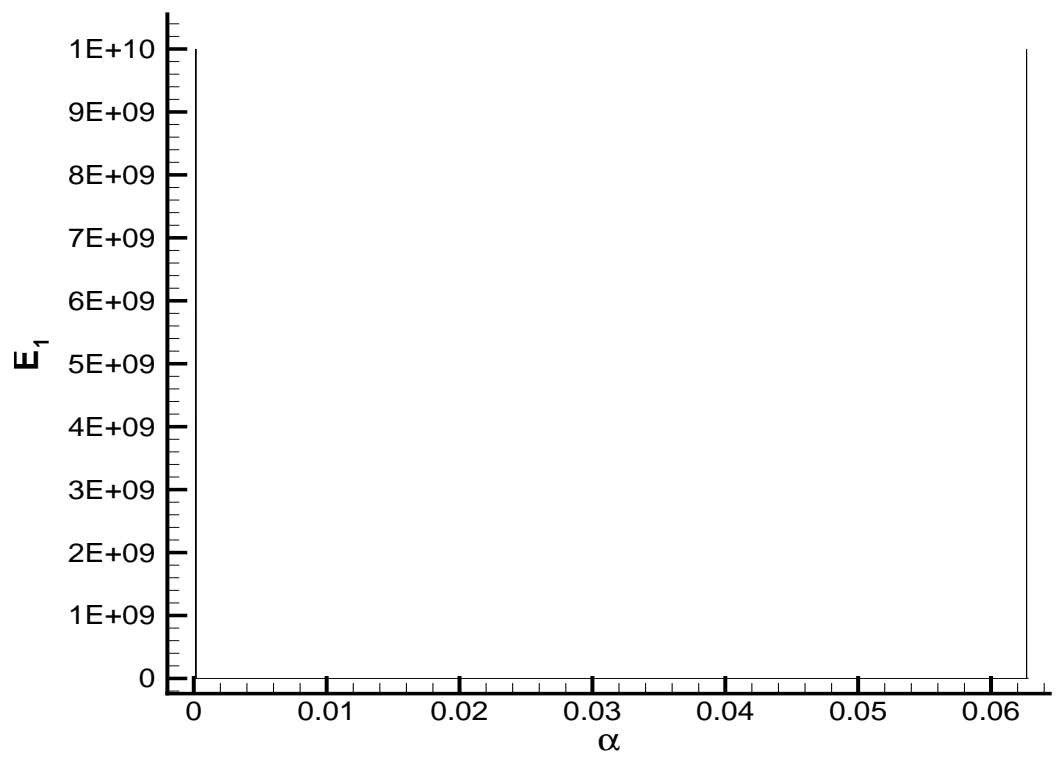

Figure C.2: The energy spectra of sinusoidal signals

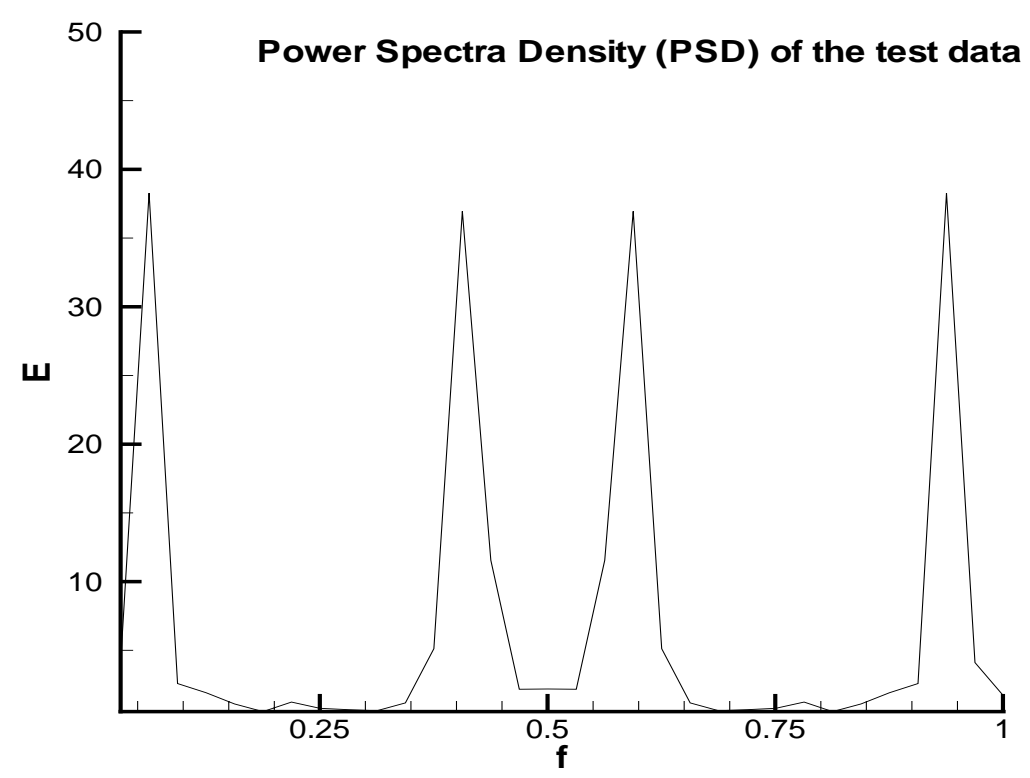

Figure C.3: The energy spectra of the test data 


\section{Bibliography}

Abe, H., Kawamura, H., and Matsuo, Y.: 2001, Direct numerical simulation of a fully developed turbulent channel flow with respect to the reynolds number dependence, Journal of Fluid Engineering 123, 382

Akselvoll, K. and Moin, P.: 1996, Large-eddy simulation of turbulent confined coannular jets, J.Fluid Mech. 315, 387

Andreopoulos, J. and Bradshaw, P.: 1980, Measurements of interacting turbulent shear layers in the near wake of a flat plate, J.Fluid Mech. 100, 639

Avancha, R. and Pletcher, R.: 2000, Large eddy simulation of the turbulent flow past a backward facing step, in 38th AIAA Aerospace Sciences Meeting and Exhibit, Reno, NV

Bardina, J.: 1989, Toward a general turbulence model, AIAA Paper (89-1862)

Beam, R. and Warming, R.: 1976, An implicit finite-difference algorithm for hyperbolic systems in conservation-law form, Journal of Computational Physics 24, 87

Bechara, W., Bailly, C., and Lafon, P.: 1994, Stochastic approach to noise modeling for free turbulent flows, AIAA Journal 32(3)

Benilov, A., Bang, G., Safray, A., and Tkachenko, I.: 2000, Ship wake detectability in the ocean turbulent environment, in Twenty-Third Symposium on Naval Hydrodynamics, Val de Reuil, France

Boris, J., Grinstein, F., Oran, E., and Kolbe, R.: 1992, New insights into large eddy 
simulation, Fluid Dynamics Research 10, 199

Bosch, G. and Rodi, W.: 1998, Simulation of vortex shedding past a square cylinder with different turbulence models, Int.J.Numer.Meth.Fluids 28, 601

Brandt, A.: 1977, Multi-level adaptive solutions to boundary-value problems, Math. Comput. 31, 333

Cabot, W. and Moin, P.: 2000, Approximate wall boundary conditions in the large-eddy simulation of high reynolds number flow, Flow, Turbulence and Combustion 63, 269

Calhoun, R.: 1998, Numerical Investigations of Turbulent Flow Over Complex Terrain, Ph.D Dissertation, Stanford University, Stanford,CA

Celik, I., Smirnov, A., and Smith, J.: 1999, Appropriate initial and boundary conditions for LES of a ship wake, in 3rd ASME/JSME Joint Fluids Engineering Conference, Vol. FEDSM99-7851, San Francisco, California

Chasnov, J. R.: 1991, Simulation of the kolmogrov inertial subrange using an improved subgrid model, Phys.Fluids A 3, 188

Clark, J.: 1968, A study of incompressible turbulent boundary layers in channel flow, Trans. ASME, Series D, J. Basic Engineering 90, 455

Clark, R., Ferziger, J., and Reynolds, W.: 1979, Evaluation of subgrid-scale models using an accurately simulated turbulent flow, J.Fluid Mech. 91, 1

C.Wilcox, D.: 1993, Turbulence Modeling for CFD, DCW Industries, Inc., La Canada, California

Dihn, Q.V., Glowinski, R. and Periaux, J.: 1984, Solving elliptic problems by domain decomposition methods with applications, in G. Birkhoff and A. Schoenstadt (ed.), Elliptic Problem Solvers II, Academic Press, New York

Domaradzki, A. and Saiki, E.: 1997, A subgrid-scale model based on the estimation of unresolved scales of turbulence, Physics of Fluids 10, 2148

Domaradzki, A., Saiki, E., and Hussaini, M.: 1997, A subgrid-scale model based on the 
estimation of unresolved scales of turbulence, Theoretical and Computational Fluid Dynamics 9, 75

Dommermuth, D., Gharib, M., Huang, H., Innis, G., Maheo, P., Novikov, E., Talcott, J., and Wyaatt, D.: 1996, Turbulent free shear flows: A comparison between numerical simulations and experimental measurements, in 21st Symposium on Naval Hydrodynamics, pp 200-215, Trondhiem, Norway

Dommermuth, D. and Novikov, E.: 1993, Direct numerical and large-eddy simulations of turbulent free-surface flows, in Proceeding of the Sixth International Conference on Numerical Ship Hydrodynamics, pp 239-270, Iowa City, IOWA

Elghobashi, S. and Lasheras, J.: 1996, Effects of gravity on sheared turbulence laden with bubbles or droplets, in NASA Conference Punlication Proceedings of the 1996 3rd Microgravity Fluid Physics Conference, Cleveland, OH, USA

Elghobashi, S. and Truesdell, G.: 1993, On the two-way interaction between homogeneous turbulence and dispersed solid particles. i: Turbulence modification, Physics of Fluids A 5, 1790

Ferziger, J.: 1983, Higher-level simulations of turbulent flows, in J. Essers (ed.), Computational Methods for Turbulent Transsonic and Viscous Flows, pp 93-183, Hemisphere Publishing Co., Springer Verlag

Ferziger, J. and Peric, M.: 1997, Computational Methods for Fluid Dynamics, Springer, Mercedesdruck, Berlin

Fung, J., Hunt, J., Malik, N., and Perkins, R.: 1992, Kinematic simulation of homogeneous turbulence by unsteady random Fourier modes, J. Fluid Mech. 236, 281

Fureby, C., Tabor, H., Weller, H., and Gosman, A.: 1997, A comparative study of subgrid scale models in homogeneous isotropic turbulence, Phys. Fluids 9(5), 1416

Germano, M.: 1986, A proposal for a redefinition of turbulent stresses in the filtered navier-stokes equation, Phys. Fluids A 29, 2323 
Germano, M., Piomelli, U., Moin, P., and Cabot, W.: 1991, A dynamic subgrid-scale eddy viscosity model, Phys. Fluids A 3, 1760

Ghosal, S., Lund, T., Moin, P., and Akselvoll, K.: 1995, A dynamic localization model for large-eddy simulation of turbulent flows, J.Fluid Mech. 286, 229

Goldberg, U.: 1991, Derivation and testing of a one-equation model based on two time scales, AIAA Journal 29, 1337

Goldberg, U. and Chakravarthy: 1990, Separated flow prediction using a hybrid $k-$ l/backflow model, AIAA Journal 28, 1005

Hayasa, T.: 1999, Monotonic convergence property of turbulent flow solution with central difference and quick schemes, Journal of Fluids Engineering 121, 351

Hoekstra, M.: 1991, Macro wake features of a range of ships, Technical Report Report No. 410461-1-PV, Maritime Research Institute Netherlands

Horiuti, K.: 1985, Large eddy simulation of turbulent channel flow by one-equation modeling, Journal of the Physical Society of Japan 54(8), 2855

Hussain, A. and Reynolds, W.: 1975, Measurements in fully developed turbulent channel flow, Transactions of the ASME, Journal of Fluids Engineering 97, 568

Hwang, C. and Lin, C.: 1998, Improved low-reynolds-number $k-\tilde{\epsilon}$ model based on direct numerical simulation data, AIAA Journal 36 (1), 38

Hyman, M.: 1995, Calculation of initial data planes for shipwake simulations, Technical Report CSS/TR-96/07, Coastal Research and Technology Department

Hyman, M.: 1998, Computation of ship wake flows with free-surface/turbulence interaction, in 22nd Symposium on Naval Hydrodynamics, pp 11-32, Washington,D.C.

Hyman, M.: 2001, Ship-Wake simulations, Private communication

Jimenez, J. and Moser, R.: 2000, Large-eddy simulations: where are we and what can we expect?, AIAA Journal 36, 605

Jin, H. and Zhang, S.: 1992, Numerical simulation of stratified flow with both vertical 
temperature gradient and vertical salinity gradient, Journal of Hydrodynamics 4, 24

Jordan, S.: 1999, A large-eddy simulation methodology in generalized curvilinear coordinates, Journal of Computational Physics 148, 322

Jordan, S.: 2001, Dynamic subgrid-scale modeling for large-eddy simulations in complex topologies, Journal of Fluids Engineering 123, 619

Jordan, S. and Ragab, S.: 1998, A large-eddy simulation of the near wake of a circular cylinder, Journal of Fluids Engineering 120, 243

Kay, S.: 1988, Modern spectral estimation, PTR Prentice Hall, Englewood Cliffs, New Jersey

Kim, J. and Moin, P.: 1985, Application of a fractional step method to incompressible navier-stokes equations, Journal of Computational Physics 59, 308

Kim, J. and Moin, P.: 1989, DNS of channel flows, ERCOFTAC database

Kim, J., Moin, P., and Moser, R.: 1987, Turbulence statistics in fully developed channel flow at low reynolds number, Journal of Fluid Mechanics 117, 133

Kim, W., Menon, S., and Mongia, H.: 1999, Large-eddy simulation of a gas turbine combustor flow, Combust. Sci. and Tech. 143, 25

Kraichnan, R.: 1970, Diffusion by a random velocity field, Phys. Fluid 11, 43

Larsson, L., Regnstrom, B., Li, D., and Janson, C.: 1998, Failures, fantasies, and feats in the theoretical/numerical prediction of ship performance, in 22nd Symposium on Naval Hydrodynamics, pp 11-32, Washington,D.C.

Lazaro, B. and Lashears, J.: 1992a, Particle dispersion in the developing free shear layer. part 1. unforced flow, J.Fluid Mech. 235, 179

Lazaro, B. and Lashears, J.: 1992b, Particle dispersion in the developing free shear layer. part 1. unforced flow, J.Fluid Mech. 235, 143

Lee, S., Lele, S., and Moin, P.: 1992, Simulation of spatially evolving turbulence and the applicability of Taylor's hypothesis in compressible flow, Physics of Fluids 4, 1521 
Leith, C.: 1990, Stochastic backscatter in a subgrid-scale model: plane shear mixing layer, Phys.Fluids A 2, 297

Leonard, B.: 1979, A stable and accurate convective modeling procedure based on quadratic upstream interpolation, Comput. Methods Appl. Mech. Engr. 19, 59

Leonard, B.: 1988, Simple high accuracy resolution program for convective modeling of discontinuities, International Journal for Numerical Methods in Fluids 8, 1291

Lewellen, D., Lewellen, W., and Xia, J.: 2000, The influence of a local swirl ratio on tornado intensification near the surface, Journal of the Atmospheric 15 (2), 527

Lewellen, W.: 1977, Use of invariant modeling, in Handbook of turbulence, Plenum

Li, A., Ahmadi, G., Bayer, R., and Gaynes, M.: 1994, Aerosol particle deposition in an obstructed turbulent duct flow, J. Aerosol Sci. 25(1), 91

Lilly, D.: 1967, The representation of small-scale turbulence in numerical experiments, in Proc. IBM Scientific Computing Symposium on Environmental Science, White Plains, NY

Liu, S., Meneveau, C., and Katz, J.: 1994a, Experimental studies of similarity subgrid-scale models of turbulence using conditional averaging, ASME FED 118, 1

Liu, S., Meneveau, C., and Katz, J.: 1994b, On the properties of similarity subgrid-scale models as deduced from measurements in a turbulent jet, J.Fluid Mech. 275, 83

Lombardi, P., De Angelis, V., and Banerjee, S.: 1996, Direct numerical simulation of near-interface turbulence in coupled gas-liquid flow, Phys. Fluids 8(2), 1643

Lund, T.: 1998, Generation of turbulent inflow data for spatially-developing boundary layer simulations, Journal of Computational Physics 140, 233

Lund, T., Wu, X., and Squires, K.: 1998, Generation of turbulent inflow data for spatiallydeveloping boundary layer simulations, Journal of Computational Physics 140, 233

Lyn, D., Einav, S., Rodi, W., and Park, J.: 1995, A laser-doppler velocimetry study of ensemble-averaged characteristics of the turbulent near wake of a square cylinder, Jour- 
nal of Fluid Mechanics 304, 285

Magnient, J., Sagaut, P., and Deville, M.: 2001, A study of built-in filter for some eddy viscosity models in large-eddy simulation, Phys. Fluids 13 (5), 1440

Mansour, N., Kim, J., and Moin, P.: 1988, Reynolds-stress and dissipation-rate budgets in a turbulent channel flow, J.Fluid Mech. 194, 15

Mason, P. J. and Thomson, D. J.: 1992, Stochastic backscatter in large-eddy simulation of boundary layers, J.Fluid Mech. 242, 51

Maxey, M.: 1987, The gravitational settling of aerosol particles in homogeneous turbulence and random flow fields, J. Fluid Mech. 174, 441

Menon, S. and Yeung, P.: 1995, Analysis of subgrid models using direct and large-eddy simulations of isotropic turbulence, in Proc. 74th AGARD/FDP Symp. on Application of Direct and Large Eddy Simulation to Transition and Turbulence, AGARD

Menon, S., Yeung, P., and Kim, W.: 1996, Effect of subgrid models on the computed interscale energy transfer in isotropic turbulence, Computers \& Fluids 25, 165

Milgram, J., Peltzer, R., and Griffin, O.: 1993, Suppression of short sea waves in ship wakes: measurements and observations, J. Geophysics Res. 98, 7103

Moin, P. and Kim, J.: 1982, Numerical investigation of turbulent channel flow, J.Fluid Mech. 118, 341

Nakayama, A. and Liu, B.: 1990, The turbulent near wake of a flat plate at low reynolds number, J.Fluid Mech. 217, 93

Naudascher, E.: 1965, Flow in the wake of self-propelled bodies and related sources of turbulence, J.Fluid Mech. 22, 625

Okamoto, M. and Shima, N.: 1999, Investigation for the one-equation-type subgrid model with eddy-viscosity expression including the shear-damping effect, JSME International Journal 42, 154

Osman, A., Ammar, H., Smirnov, A., Shi, S., and Celik, I.: 2000, Parallel analysis and 
implementation of large eddy simulations of ship wakes, in IEEE International Conference on Cluster Computing CLUSTER 2000 Technische Universitt Chemnitz, Saxony,Germany

Paterson, E., Hyman, M., Stern, F., P., C., Bonetto, F., Drew, D., and Lahey, R.: 1996, Near- and far-field cfd for a naval combatant including thermal-stratification and twofluid modeling, in 21st Symposium on Naval Hydrodynamics, pp 102-117, Trondheim, Norway

Paterson, E., Wilson, R., and Stern, F.: 1998, Verification/validation of steady flow rans cfd for naval combatant, in 1st Marine CFD Applications Symposium, Washington,D.C.

Perng, C.: 1990, Adaptive-multigrid computations for incompressible flows, including geometry, temperature, and salinity effects, Ph.D Dissertation, Stanford University, Stanford,CA

Piomelli, U.: 1997, On the effects of nonequilibrium on the subgrid-scale stresses, Phys. Fluids 9 (9), 2740

Piomelli, U.: 1998, Large eddy simulations: Present state and future directions, AIAA Paper 980534

Piomelli, U.: 1999a, High reynolds number calculations using the dynamic subgrid-scale stress model, Phys. Fluids A 5, 1484

Piomelli, U.: 1999b, Large-eddy simulation: achievements and challenges, Progress in Aerospace Sciences 35, 335

Piomelli, U., Cabot, W., Moin, P., and Lee, S.: 1991, Subgrid-scale backscatter in turbulent and transitional flows, Phys.Fluids A 3, 1766

Piomelli, U., Ferziger, J., and Moin, P.: 1988, Models for LES of turbulent channel flows including transpiration, Technical Report TF-32, Department of Mechanical Engineering, Stanford University

Pomraning, E. and Rutland, C.: 2001, A dynamic one-equation non-viscosity LES model, 
submitted to Phys. Fluids

Rai, M. and Moin, P.: 1991, Direct simulation of turbulent flows using finite-difference schemes, Journal of comutational physics 109 (2), 169

Ramaprian, B. and Patel, V.: 1982, The symmetric turbulent wake of a flat plate, AIAA Journal 20, 1228

Ramaprian, B., Patel, V., and Sastry, M.: 1981, Turbulent wake development behind streamlined bodies, Technical Report IIHR Report No.231, Iowa Institute of Hydraulic Research, The University of IOWA

Rashidi, M., Hetsroni, G., and Banerjee, S.: 1992, Wave-turbulence interaction in freesurface channel flows, Phys. Fluids A4(12), 2727

Ratcliffe, T.: 1998, Validation of free surface reynolds averaged navier stokes and potential flow codes, in 22nd Symposium on Naval Hydrodynamics, pp 964-980, Washington,D.C.

Ravikanth, V. and Pletcher, R.: 2000, AIAA Paper (2000-0542)

Reed, A., Beck, R., Griffin, O., and Peltzer, R.: 1990, Hydrodynamics of remotely sensed surface ship wakes, SNAME Transactions 98, 319

Rightley, P.: 1995, Bubble dispersion and interphase coupling in a free shear flows, Ph.D Dissertation, University of California, San Diego,CA

Rodi, W.: 1997, Large-eddy simulations of the flow past bluff bodies: State-of the art, in Proc. int.Conf.Fluid Engineering, JSME Centennial Grand Congress, Tokyo

Salvetti, M. and Banerjee, S.: 1995, A priori tests of a new dynamic subgrid-scale model for finite-difference large-eddy simulations, Phys. Fluids 7, 2831

Salvetti, M., Zang, Y., Street, R., and Banerjee, S.: 1997, Large-eddy simulation of freesurface decaying turbulence with dynamic subgrid-scale models, Phys. Fluids $\mathbf{9 ( 8 ) ,}$ 2405

Schumann, U.: 1975, Subgrid scale model for finite difference simulation of turbulence 
flows in plane channels and annuli, Journal of Computational Physics 18, 376

Shi, S., Celik, I., and Smirnov, A.: 2000a, Comparison of different numerical schemes and sub-grid scale models in large-eddy simulation, in ASME Fluids Engineering Division Summer Meeting, No. 11232 in FEDSM2000, Boston,Massachusetts

Shi, S., Smirnov, A., and Celik, I.: 2000b, An approach for generating time-dependent inflow boundary with application to large-eddy simulation of flat plate wake, in International Mechanical Engineering Congress and Exposition, Orlando, FL

Shi, S., Smirnov, A., and Celik, I.: 2000c, Large eddy simulations of particle-laden turbulent wakes using a random flow generation technique, in ONR 2000 Free Surface Turbulence and Bubbly Flows Workshop, pp 13.1-13.7, California Institute of Technology, Pasadena, CA

Simon, H.D. (ed.): 1992, Parallel Computational Fluid Dynamics: Implementations and Results Using Parallel Computers, MIT Press, Cambridge

Smagorinsky, J.: 1963, General circulation experiments with the primitive equations, part i : the basic experiment, Monthly Weather Rev. 91, 99

Smirnov, A., Shi, S., and Celik, I.: 2000a, Random flow generation technique for large eddy simulations and particle-dynamics modeling, Journal of Fluids Engineering, Submitted for publication, March 31, 2000, Log No. 5367-GEK

Smirnov, A., Shi, S., and Celik, I.: 2000b, Random flow simulations with a bubble dynamics model, in ASME Fluids Engineering Division Summer Meeting, No. 11215 in FEDSM2000, Boston,Massachusetts

Smirnov, A., Shi, S., and Celik, I.: 2001a, Bubble tracking in a ship wake, in 2001 ASME Fluids Engineering Division Summer Meeting, New Orleans

Smirnov, A., Shi, S., and Celik, I.: 2001b, Random flow generation technique in large eddy simulations and particle-dynamics modeling, Journal of Fluid Engineering 123, 359

Sohankar, A., Davidson, L., and Norberg, C.: 2000, Large eddy simulation of flow past 
a square cylinder: Comparison of different subgrid scale models, Journal of Fluids Engineering 122, 39

Sotiropoulos, F. and Patel, V.: 1995, Application of reynolds-stress transport models of stern and wake flows, Journal of Ship Research 39, 263

Spain, B.: 1965, Tensor Calculus, Oliver and Boyd

Stern, F., Longo, J., Oliveieri, A., and Coleman, H.: 2000, International collaboration on benchmark cfd validation data for surface combatant dtmb model 5415, in Twenty-Third Symposium on Naval Hydrodynamics, Val de Reuil, France

Stern, F. and Wilson, R.: 2000, RANS Results of Ship-Wake simulations, Private communication

Tennekes, H. and Lumley, J.: 1997, A first course in turbulence, The MIT press, Cambridge, Massachusetts

Truesdell, G. and Elghobashi, S.: 1994, On the two-way interaction between homogeneous turbulence and dispersed solid particles. ii: Particle dispersion, Physics of Fluids 6, 1405

Vasilyev, O., Lund, T., and Moin, P.: 1998, A general class of commutative filters for les in complex geometries, Journal of Computational Physics 146, 82

Voke, P. and Potamitis, S.: 1994, Numerical simulation of a low-reynolds-number turbulent wake behind a flat plate, International Journal for Numerical Methods in Fluids 19, 377

Vreman, B., Geurts, B., and Kuerten, H.: 1997, Large-eddy simulation of the turbulent mixing layer, J.Fluid Mech. 339, 357

Wang, M. and Moin, P.: 2000, Computation of trailing-edge flow and noise using largeeddy simulation, AIAA Journal 12, 2201

Wygnanski, I., Champagne, F., and Marasli, B.: 1986, On the large-scale structures in two-dimensional, small-deficit , turbulent wakes, J.Fluid Mech. 168, 31

Yoshizawa, A. and Horiuti, K.: 1985, A statistically-derived subgrid-scale kinetic energy 
model for the large-eddy simulation of turbulent flows, Journal of the Physical Society of Japan 54(8), 2834

Zang, Y.: 1993, On The Development of Tools For The Simulation of Geophysical Flows, Ph.D Dissertation, Stanford University, Stanford,CA

Zang, Y., Street, R., and Koseff, J.: 1993, A dynamic mixed subgrid-scale model and its application to turbulent recirculating flows, Phys. Fluids 5-12, 3186

Zang, Y., Street, R., and Koseff, J.: 1994, A non-staggered grid, fractional step method for time-dependent incompressible navier-stokes equations in curvilinear coordinates, Journal of Computational Physics 114, 18

Zhou, O. and Leschziner, M.: Sept. 1991, A time-correlated stochastic model for particle dispersion in anisotropic turbulence, in 8-th Turbulent Shear Flows Symp., Munich

Zhou, Q. and Leschziner, M.: 1996, Modelling particle Dispersion in Turbulent Recirculating Flow with an Anisotropy- Resolving Scheme, Technical Report TFD/96/07, UMIST 